\title{
Propiedades electrocinéticas \\ y estructurales de electrolitos confinados \\ en nanoporos de forma cilíndrica o plana.
}

Tesis que presenta

Enrique Sánchez Arellano

Para obtener el grado de

Doctor en Ciencias

Asesor: $\quad$ Dr. Marcelo Lozada y Cassou

Jurado Calificador:

Presidente: Dr. Rolando Castillo Caballero

UNAM

Secretario: Marcelo Galván Espinosa

UAM-I

Vocal:

Eugenia Corvera Poiré

Vocal:

Marcelo Lozada y Cassou

UNAM

Vocal:

José Alejandre Ramírez

IMP

UAM-I

México, D. F. Agosto 2010 


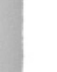$$
\text { . }
$$

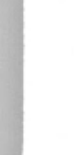




\section{Índice general}

Agradecimientos $\quad \mathrm{v}$

Prefacio $\quad$ VII

Introducción General $\quad$ IX

1. Teoría general $\quad 1$

1.1. Teoría de transporte .......................... 1

1.1.1. Variables electrocinéticas ...................... 5

1.2. Cuerpos Sumergidos en un Electrolito . . . . . . . . . . . 6

1.2.1. Conceptos Generales .................. 6

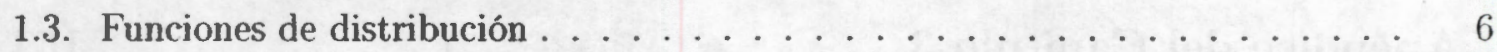

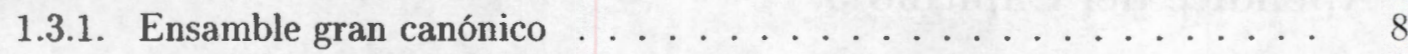

1.4. La ecuación de Ornstein Zernike . . . . . . . . . . . . . 10

1.4.1. El modelo primitivo. . . . . . . . . . . . . . . . 11

1.4.2. Fluidos inhomogéneos: el método directo ............ 11

1.4.3. La ecuación de Poisson-Boltzmann . . . . . . . . . . . . 13

1.4.4. Modelo de Gouy-Chapman . . . . . . . . . . . . . . 15

1.5. Fluidos en Equilibrio . . . . . . . . . . . . . . . . . 15

1.6. Fuerza entre dos cuerpos cargados . . . . . . . . . . . . . . . 17

1.6.1. La carga Inducida en un Fluido . . . . . . . . . . . . . 21

2. Efecto de la geometria en las propiedades electrocinèticas de electrolitos confinados en poros de forma cilíndrica o de rendija plana.

2.1. Introducción . . . . . . . . . . . . . . 25

2.2. La teoría y los modelos . . . . . . . . . . . . . . 27

2.2.1. Teoría general HNC-MSA para una solución iónica en un poro cargado 28

2.2.2. Teoría HNC/MSA para una solución iónica en un poro cargado de rendija plana ............................ 29

2.2.3. Teoría HNC-MSA para una solución iónica en un poro cargado de forma cilíndrica . . . . . . . . . . . . 30

2.2.4. Casos Límites de tamaño de poros infinitos e iones puntuales . . . . 32

2.2.5. Ecuaciones para las propiedades electrocinéticas para un microporo de forma de rendija de 2 placas . . . . . . . . . . . . 33 
2.2.6. Ecuaciones para las propiedades electrocinéticas para un microporo

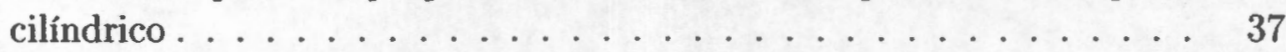

2.2.7. Resultados y Discusión .................... 42

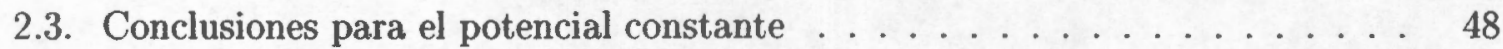

2.4. Conclusiones, para carga constante ... . . . . . . . . 56

3. Interacción efectiva entre Cuatro Placas Planas Paralelas Cargadas inmersas en un Electrolito $\quad 59$

3.1. Introducción . . . . . . . . . . . . . . . . . . . 59

3.2. Teoría de Funciones de Distribución . . . . . . . . . . . . 65

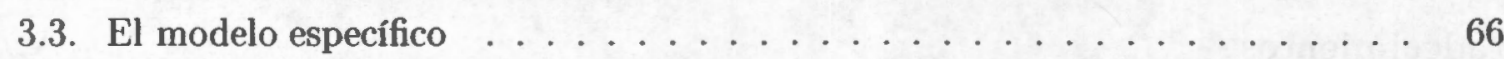

3.4. Carga Inducida en el Fluido . . . . . . . . . . . . . . . . . 69

3.5. La presión neta sobre las placas. . . . . . . . . . . . . 71

3.6. Análisis de Resultados . . . . . . . . . . . . . . . 72

3.7. Conclusiones del capítulo $3 \ldots \ldots \ldots \ldots \ldots$

3.8. Conclusiones Generales .................... 93

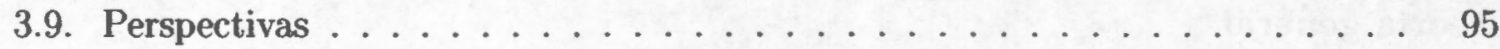

I Apéndice del Capítulo 2 $\quad 97$

$\begin{array}{ll}\text { II Apéndice del Capítulo 3 } & 101\end{array}$

A. La función de distribución radial, para las Cuatro Placas 103

B. Desarrollo Analitico de los Kernels de la Función de Distribución Radial, $\begin{array}{ll}\text { para las Cuatro Placas } & 107\end{array}$

$\begin{array}{ll}\text { C. La Fuerza sobre las placas } & 111\end{array}$

D. Ecuaciones para el Potencial Electrostático, para el caso de Las Cuatro Placas

E. Cálculo de la Presión Osmótica $\quad 121$

$\begin{array}{ll}\text { F. Método Numérico. } & 125\end{array}$

$\begin{array}{lr}\text { G. Gráficas } & 129\end{array}$ 


\section{Agradecimientos}

Quiero agradecer a todas las personas que me ayudaron y motivaron para terminar este trabajo, en especial a:

Dr. Marcelo Lozada y Cassou, director de esta tesis por su apoyo, paciencia y confianza.

Dr. Felipe Jiménez Ángeles por su apoyo en todos los aspectos y para revisar la tesis.

Familiares y amigos.

También quiero agradecer a la UAM-I, por apoyarme y darme una oportunidad de salir adelante, al CONACYT, al IPN; así como al IMP, por permitirme trabajar en sus instalaciones. 
vi 


\section{Prefacio}

En este trabajo presentamos la solución de tres sistemas de fluidos cargados, confinados (entre dos placas, en un cilindro y entre cuatro placas). De manera general, se presenta la teoría que usaremos y se resuelve un primer problema que consiste en comparar como se comportan los iones de un electrolito dentro de dos poros de geometría diferente. En éstos se estudian las propiedades electrocinéticas (tales como conductancia capilar, electroviscosidad, y potencial de corriente) que se obtienen para el modelo primitivo restringido, de un electrolito confinado; en un caso, en un poro de rendija plana, formado por dos placas paralelas, y en otro caso en una cavidad cilíndrica de diámetro finito. Ambos poros están cargados e inmersos en el fluido cargado.

La teoría HNC/MSA (aproximación esférica media de cadena hipertejida), es usada para obtener los perfiles de concentración iónica en equilibrio, dentro de los poros, que a la vez, son usadas para calcular las propiedades electrocinéticas por medio de ecuaciones hidrodinámicas lineales. Los resultados obtenidos son comparados con los de la teoría clásica de PoissonBoltzmann (PB). Los efectos cuantitativos y cualitativos, atribuidos a la geometría y a la consideración de las correlaciones de corto alcance de HNC/MSA, se discuten al final.

Por último, de acuerdo a la teoría de ecuaciones integrales para un fluido inhomogéneo, se presenta un desarrollo con la teoría HNC/MSA, para un sistema de 4 placas paralelas infinitas, separadas una distancia finita, de un cierto espesor, cargadas e inmersas en un electrolito. Se analizan efectos como la estructura elemental, las fuerzas sobre las placas y los efectos de adsorción conectados con un orden en el acomodo de los iones del electrolito. Para los cálculos, se elaboran la teoría y los programas específicos para estos problemas. 
VIII 


\section{Introducción General}

Un electrolito es un fluido que está formado de partículas cargadas llamadas iones; las cuales pueden ser de varias especies con diferentes signos de carga (positivos y negativos) y tamaño. El sistema es neutro de manera global. El estudio de fluidos cargados en presencia de partículas coloidales y/o interfases es de gran interés en diversos campos de investigación tanto básica como aplicada $[1,2]$. En presencia de una macropartícula cargada o de un campo externo, la concentración local de una solución electrolítica homogénea se ve afectada y se crea una inhomogeneidad en la vecindad del campo o macropartícula. Se observa que los iones de carga opuesta a la macropartícula (conocidos como contraiones), se sienten atraídos hacia la superficie cargada por lo que tienen una concentración alta cerca de la pared, que disminuye a medida que la distancia a ésta se incrementa. Los otros iones que tienen carga del mismo signo que la pared (y se conocen como coiones) son rechazados por esta superficie, formando un perfil de concentración tal que entre más cerca estén de la pared menor es su concentración. A este perfil de concentración de carga formado en la vecindad de una superficie cargada se le llama doble capa eléctrica (DCE). Se puede observar también que a distancias grandes de la superficie cargada, la concentración de las especies es uniforme, siempre y cuando no exista una perturbación producida por otra macropartícula. Los iones en el fluido se distribuyen de manera inhomogénea cerca de la pared y tienden a neutralizar la carga de la pared. Sin embargo, puede ocurrir que los contraiones no sólo compensen la carga superficial efectiva de la superficie, sino que bajo ciertas condiciones puedan sobrecompensarla causando un cambio de signo, este fenómeno es llamado inversión de carga (CI).

El interés por la doble capa eléctrica es importante porque, existen sistemas con fluidos cargados confinados por paredes cargadas, donde su estudio es relevante. Por ejemplo, en el campo de la biofísica son de importancia los poros, canales y cavidades de dimensiones nanométricas que, por ejemplo, interconectan el interior y el exterior de las membranas celulares. Estos canales pueden considerarse, en una aproximación muy simple, como poros de geometría plana o cilíndrica. Para puntualizar, en este trabajo nos referiremos a un fluido confinado como aquel que está restringido a moverse en alguna de sus dimensiones, i.e., dent ro de una región específica, como por ejemplo, un fluido entre partículas coloidales cercanas entre sí. El confinamiento incluye aquellos fluidos que se encuentran rodeando objetos, por ejemplo, un fluido cerca de un coloide o fluidos internos dentro de poros, cavidades y rendijas. Inicialmente calculamos los perfiles de concentración de iones en ün electrolito confinado entre una rendija plana formada por dos placas planas infinitas y dentro de un cilindro infinitamente largo, cada una de estas superficies inmersas en un electrolito y por separado.

El término coloide se refiere a una particula que se encuentra dispersa en el seno de 
un medio que puede considerarse continuo y el tamaño de las partículas dispersas tiene aproximadamente dimensiones entre un nanómetro y un micrómetro.

La ciencia de coloides es interdisciplinaria y sus campos de interés abarcan la Física, la Biología, la Ciencia de Materiales y otras disciplinas. El estudio de los sistemas coloidales $\{3,4,5,6]$, membranas biológicas $[7]$ y electrodos inmersos en fluidos electrolíticos puede ser abordado desde el punto de vista de las interfases. Esto es, dado el tamaño de las partículas coloidales, éstas se pueden tratar como partes separadas llamadas fases. En estos sistemas de más de una fase, los coloides y la ciencia de superficies convergen. La palabra superficie es usada en el sentido físico más que el sentido geométrico. Geométricamente, una superficie tiene área pero no espesor, mientras que físicamente divide dos regiones contiguas en las cuales las propiedades varían de una fase a otra. Esta transición ocurre en distancias muy pequeñas, entonces una superficie tiene un espesor que podemos imaginar muy pequeño. El término interfase se usa en este contexto y se usa para enfatizar el hecho de que la superficie de interés es la región divisoría entre dos fases. Es importante notar la relación que existe entre las partículas coloidales y el medio en que se encuentran inmersas.

En este trabajo, usaremos los términos fase continua $\mathrm{y}$ fase dispersa para referirnos al medio y a las partículas coloidales, respectivamente. La interacción entre las componentes de dos fases da origen a las propiedades de un sistema coloidal. Los factores que contribuyen a la naturaleza de estos sistemas son: el tamaño y forma de las partículas, las propiedades de las superficies, la fase continua, las interacciones partícula-partícula, las interacciones partícula-fase continua, las diferentes fuerzas que entran en las interacciones (repulsión eléctrica, fuerzas atractivas, fuerzas viscosas, etc). En los fluidos cargados las interacciones de largo alcance y los efectos resultantes de muchos cuerpos se combinan dando lugar a fenómenos interesantes. Las interacciones electrostáticas en sistemas de muchos cuerpos en sistemas complejos y simples son importantes. En estos sistemas existe aún discrepancia en cuanto a su descripción, que es difícil debido a que las correlaciones de largo alcance y de corto alcance son relevantes. Un fluido complejo cargado es aquel en que en la fase dispersa hay iones de varias especies y tamaños. En un fluido simple cargado los iones son del mismo tamaño y sólo tenemos dos especies.

En este trabajo estudiamos un sistema formado por dos especies iónicas, con diferente carga, las cuales se encuentran dentro de un disolvente, cerca de un campo formado por una partícula, cuya densidad tiende a cero. Esta última partícula es un poro plano formado por dos placas paralelas o un poro cilíndrico, como modelo de coloide.

Si un fluido es obligado a moverse en un conducto limitado por paredes sólidas, siempre se produce un cierto rozamiento entre las distintas capas del fluido y entre éstas y las paredes de los conductos. Los efectos del rozamiento en el movimiento de fluidos se cuantifican a través del concepto de viscosidad. Los líquidos muy viscosos de mueven muy lento, mientras que los poco viscosos se mueven más rápido. También en un fluido en movimiento se producen fuerzas de arrastre sobre los objetos que hay en el seno del fluido. Si en diferentes partes de estos sistemas existen condiciones físicas distintas, la tendencia al equilibrio produce un cambio que trata de igualar dichas condiciones. El desplazamiento de las cargas del fluido se clasifica en lo que denominamos fenómenos de transporte [1]. Los ejemplos más importantes de fenómenos de transporte son el movimiento de partículas ante una variación de su concentración y el paso de carga o conducción eléctrica ante una diferencia de potencial en un medio inhomogéneo. Lo primero que queremos hacer es cuantificar la cantidad de partículas, o de carga eléctrica que 
es trasladada en un proceso de transporte. Para ello nos posicionamos en una determinada superficie, (puede ser imaginaria) y medimos el número de partículas o carga que la atraviesa por unidad de tiempo, definimos esta cantidad como el flujo correspondiente. Cuando se tiene fluido cargado en movimiento, se genera una corriente eléctrica en el mismo flujo, y se dice que se tiene un flujo electrocinético [8], que a la vez da origen a las propiedades electrocinéticas. La contribución elécrica a la resistencia al movimiento de las partículas origina un efecto viscoso adicional, conocido como efecto electroviscoso. Además, se puede definir una superficie imaginaria cuando las partículas se mueven cerca de una pared cargada donde se deslizan (plano de deslizamiento) sin tocar la pared cargada. Esta superficie se encuentra entre la parte difusa de la doble capa y la parte muy cercana a la pared cargada a una distancia de dimensiones iónicas. El potencial eléctrico en esta región se le llama el potencial $\zeta$ (zeta), y puede ser medido con técnicas experimentales. Los fenómenos de transporte son importantes porque nos permiten conocer como fluye un fluido cargado a través de un conducto que lo confina y esto puede ser usado para estimar el efecto del fluido sobre las paredes, por ejemplo, en sistemas de filtración, como consecuencia de la capilaridad o en suelos para agricultura, donde hay retención de agua. La estructura en equilibrio determina la presión neta sobre las superficies cargadas y en el electrolito $[9,10,11]$. Podemos plantearnos que un fluido simple puede moverse con una velocidad constante (flujo en estado estacionario), conocido como flujo laminar. En este trabajo estudiamos un fluido que se desplaza con velocidad constante de forma laminar dentro de un poro.

La presión osmótica es proporcional a la concentración local, ésta es muy importante, por ejemplo, es responsable de la expansión (hinchamiento) de los minerales de arcilla que pueden tener un profundo efecto en la conducta de suelos de agricultura y cimientos de construcción. La presión sobre las paredes de un poro, en forma de rendija con cuatro placas es calculada en este trabajo.

Cuando las moléculas se acomodan por sí mismas formando algúna estructura ordenada. se dice que forman un sistema autoensamblado. Éste puede ser un ensamblaje regular de pequeñas entidades moleculares en estructuras supramoleculares más grandes que exhiben nuevas funciones, que pueden no exhibirse por las moléculas en forma aislada. Un ejemplo de estruct uras supramoleculares autoensambladas son las membranas de liposomas. Existe un gran interés en conocer la naturaleza de las fuerzas $[12,13,14,15,16,17,18]$ que mantienen la estabilidad de estos sistemas [19], con la tendencia de formar planos laminados de grupos de moléculas en paquetes que se acomodan unos contra otros. La interacción entre subunidades de este tipo se debe a fuerzas electrostáticas y entrópicas. Sin embargo, poco es conocido de estas interacciones y de su naturaleza física. Este tipo de sistemas son importantes pues en el nivel más básico de todo organismo vivo hay sistemas autoensamblados. Otros ejemplos de planos paralelos supramolecularmente autoensamblados están relacionados con arcillas [20]. Estas últimas adsorben moléculas y tienen un área grande en relación con sus volúmenes, produciendo una gran superficie en un espacio pequeño.

En este trabajo estudiamos un sistema en base a un modelo formado por cuatro placas sólidas cargadas infinitas inmersas en una solución electrolítica

Los estudios antes mencionados, son un motivo para continuar estudiando estos temas pero con sistemas que tienen una estructura con más de dos placas paralelas planas. El modelo de dos placas paralelas cargadas. inmersas en un electrolito $[21,22,23,24,25]$ ha sido útil para entender sistemas tales como dispersiones coloidales $[3,4,5 \mid$, membranas biológicas $|\bar{i}|$ 
y electrodos $[4,26,27,28,29,30,31,32]$. En estos sistemas uno de los componentes es una solución que contiene iones libres, entre las placas y fuera de ellas, con una distribución en el espacio diferente, para iones positivos y negativos cerca de las placas. El estudio de las propiedades del fluido, se hace a través de la estructura de la distribución de los iones alrededor de las paredes o la doble capa eléctrica (DCE) $[33,24,34,35,36,37]$.

El orden en que está estructurado este trabajo es:

a) Síntesis de aspectos teóricos importantes relacionados con el tema a estudiar.

b) Presentación de un problema desde la perspectiva de poros infinitos, donde derivamos las propiedades de transporte iónico dentro de un cilindro, y comparamos con las propiedades de transporte de un fluido cargado confinado entre dos placas paralelas infinitas $\mid 9,10,11\}$. ya estudiado anteriormente. Observese la figura 1.

c) Estudio de la estructura de un fluido cargado, confinado por cuatro placas paralelas, infinitas y cargadas, donde calculamos los perfiles de concentración de iones del fluido o funciones de distribución de la doble capa eléctrica, y otras propiedades del sistema. Ver Figura 2, que representa este modelo.

d) Por último se presentan los apéndices, correspondientes a la teoría utilizada. $[38,39$, $40,26 \mid$. 


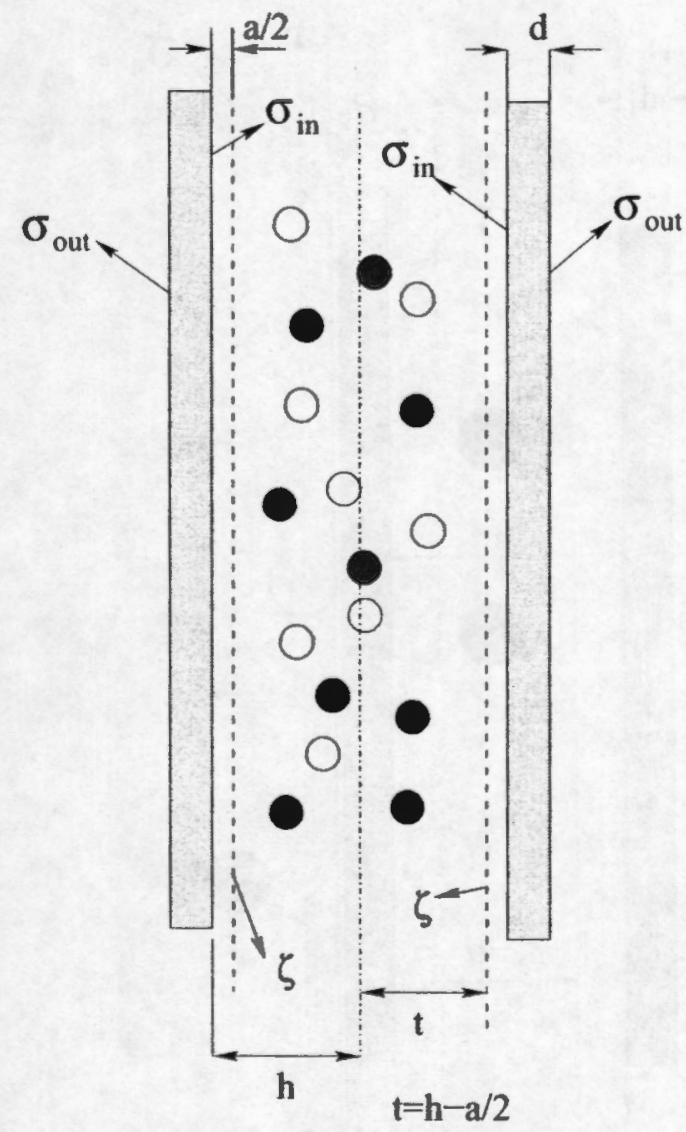

poro de dos placas paralelas

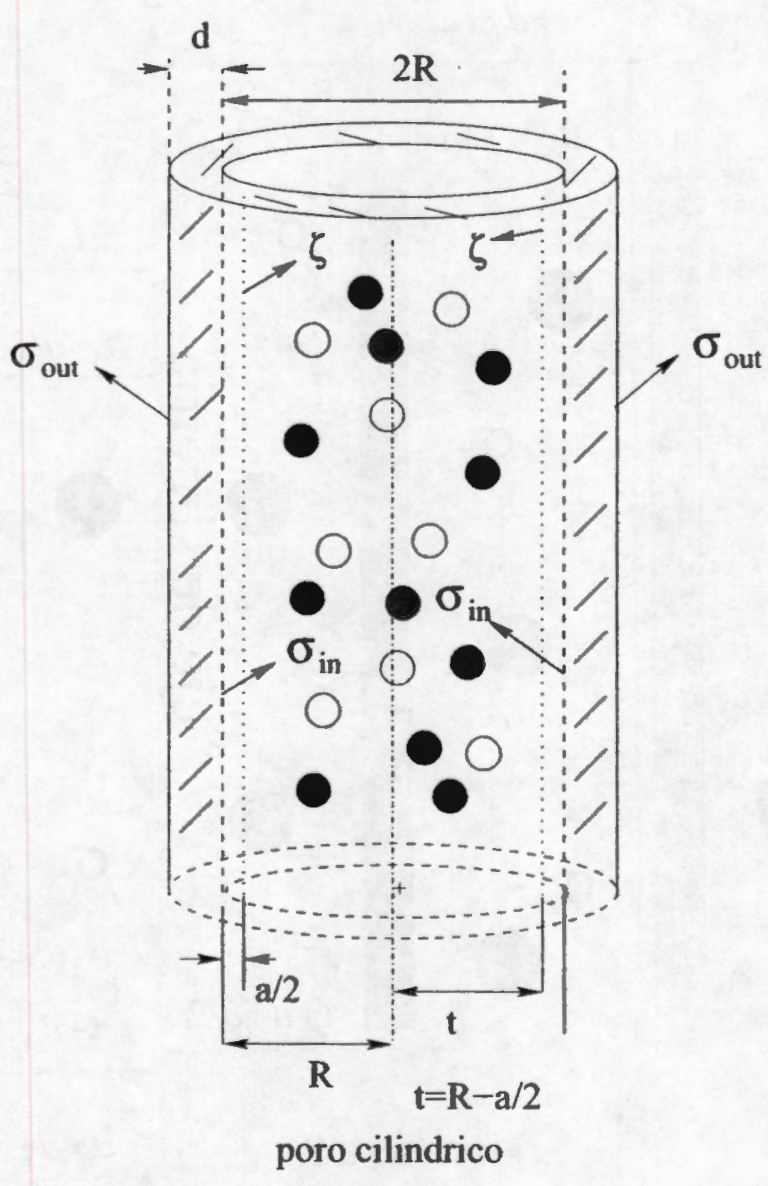

Figura 1: Figura que muestra las dos geometrías de los poros 


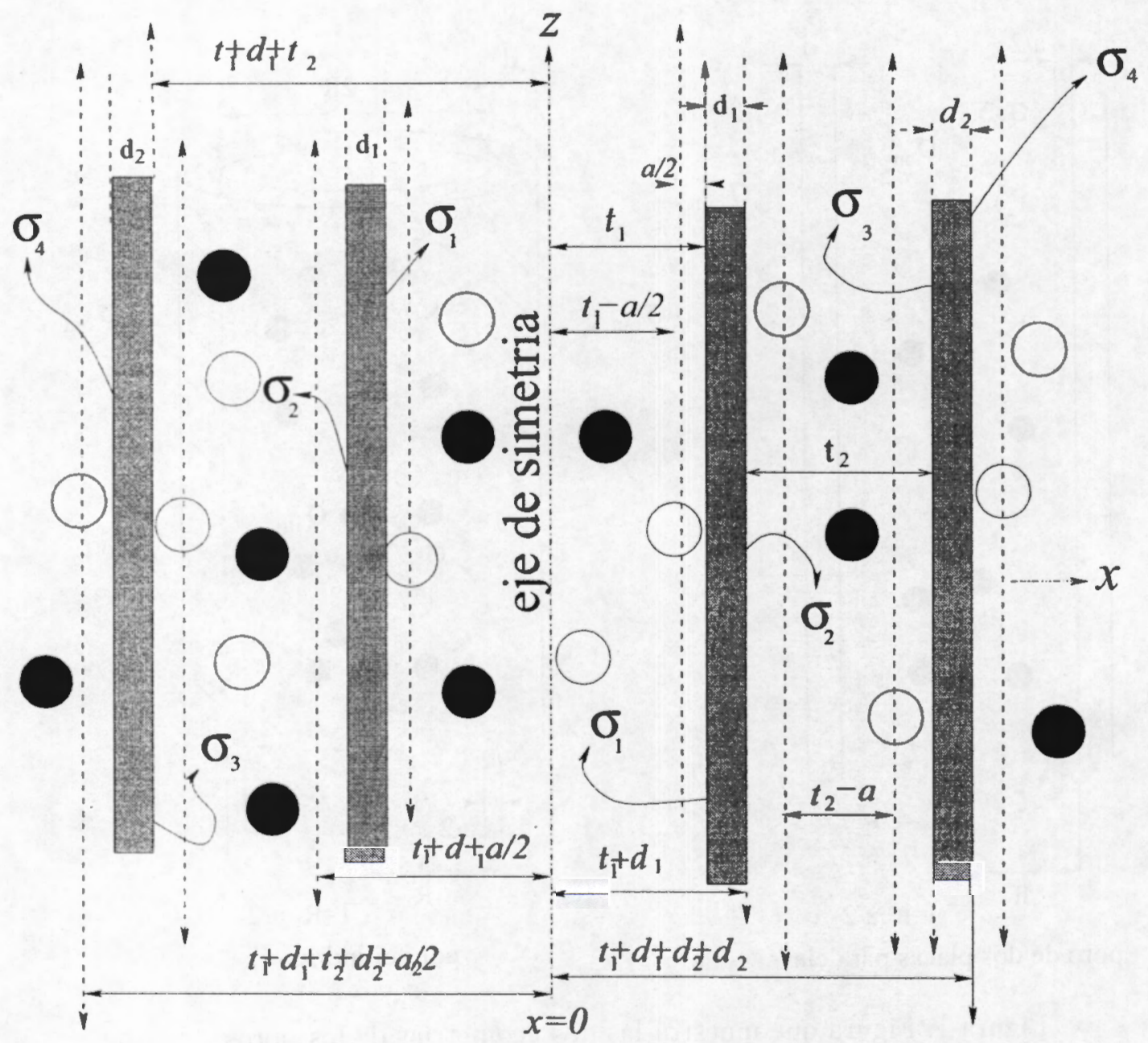

Figura 2: Figura que muestra el modelo de un poro de 4 placas 


\section{Capítulo 1}

\section{Teoría general}

\subsection{Teoría de transporte}

Una de las propiedades de los líquidos es la capacidad de flujo o de fluir en el espacio y existen ciertas razones estructurales por las que diferentes líquidos fluyen con índices de velocidad y volumen distintos. La viscosidad de un líquido es una medida de su resistencia al flujo debida a las fuerzas internas de fricción. Esta resistencia interna da como resultado una transferencia de la energía de traslación de una capa de líquido a la siguiente capa de líquido, cuando el fluido se pone en movimiento por una fuerza que se aplica sobre éste (fluido laminar). El resultado neto es una transferencia de momento entre capas sucesivas del líquido. Supongamos un líquido entre dos placas paralelas, como se muestra en la fig. 1.1 donde la fuerza tangencial o de corte $\mathrm{F}$ se aplica a las placas imaginarias o capas superiores y se deja que la inferior permanezca estacionaria.

Como se ve en la ilustración de la Fig. 1.1, la capa de líquido adyacente a la placa superior se mueve con la velocidad de la placa. Ésta a su vez arrastra a la siguiente capa inferior, y transfiere a dicha capa parte de su momento, haciendo que se desplace con una velocidad ligeramente menor que la de la capa superior. De esta manera debido a la viscosidad, cada capa arrastra a la capa inmediata inferior y hace que se desplace con menor velocidad hasta

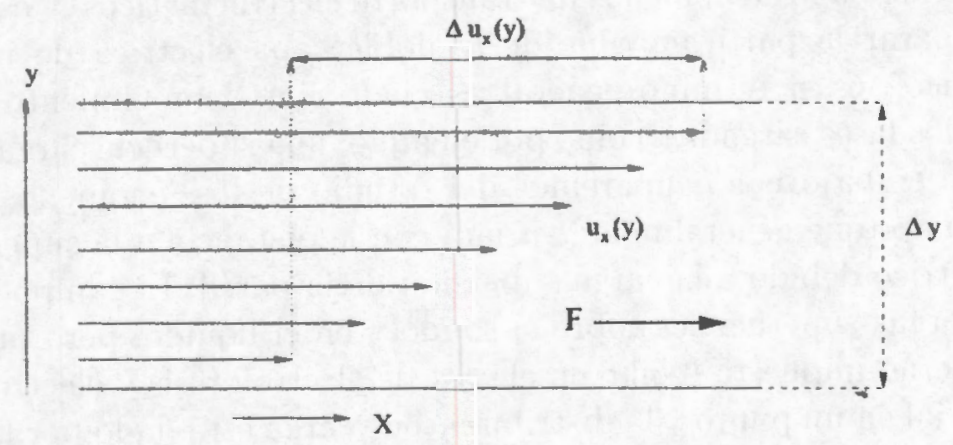

Figura 1.1: Flujo de un líquido entre placas paralelas 
que alcanza a la capa de líquido adyacente a la placa inferior que carece de movimiento. Entonces para la mayor parte de los líquidos se observa que la fuerza por unidad de área $F / A$ necesaria para impulsar a una capa de líquido en relación a la siguiente capa, es proporcional a la razón de cambio de la velocidad entre el cambio de distancia perpendicular al flujo, $\Delta u / \Delta y$, que se denomina gradiente de velocidad, así

$$
F / A=\eta \frac{\Delta u}{\Delta y}
$$

donde $u=u_{x}(y)$ y $\eta$ es la constante de proporcionalidad llamada viscosidad. Los líquidos que obedecen la ecuación anterior se llaman líquidos newtonianos. A este flujo se le llama laminar. La unidad para medir $\eta$ se llama Poise dado en $g \cdot s^{-1} \cdot \mathrm{cm}^{-1}$ (en el sistema $\mathrm{cgs}$ ).

Cuando se hace pasar un líquido por un tubo largo y delgado, por ejemplo un tubo capilar de vidrio, el flujo es afectado por la viscosidad del líquido. En 1842 Poiseuille utilizó la ecuación anterior para desarrollar una ecuación que permite calcular el coeficiente de viscosidad en el caso de flujo laminar en un tubo capilar cilíndrico. Esta ecuación es

$$
\eta=\frac{\pi r^{4} t P}{8 L V}
$$

donde $r$ es el radio del tubo delgado de longitud $L$ por el que fluye un volumen de líquido $V$, por unidad de tiempo $t$, con una presión $P$. La viscosidad relativa se define como, $\eta_{r}=\frac{\eta}{\eta_{0}}$ donde $\eta$ y $\eta_{0}$ son las viscosidades de la disolución cerca y lejos de la superficie respectivamente, $\eta_{0}$ es la viscosidad en el bulto.

En el caso de fluidos cargados en contacto con una superficie sólida estática, el líquido se mueve en respuesta a un campo eléctrico externo aplicado. A este fenómeno se le llama electroósmosis, y es de particular relevancia cuando la superficie sólida está en forma de .capilar o poro que se llena con el líquido. La medida de la velocidad del líquido, o el volumen de líquido transportado por unidad de flujo de corriente provee información acerca de la carga superficial neta o del potencial eléctrico en la vecindad de la pared (potencial $\zeta$ ).

En casi todos los fenómenos electrocinéticos es de interés el estudio del movimiento de un fluido con respecto a una superficie sólida o pared. El desplazamiento de la porción móvil de la doble capa eléctrica en respuesta a un gradiente de presión externo, y/o a un campo eléctrico externo o inducido se refiere a los fenómenos de transporte electrocinéticos, y están presentes cuando intentamos separar la parte movible de la doble capa eléctrica de una superficie cargada. "Electrocinético". es el término general asociado con el movimiento relativo a la región del bulto entre dos fases cargadas, como por ejemplo, una superficie cargada y su doble capa eléctrica. En este trabajo nos ocuparemos del estudio de los fenómenos relacionados con la velocidad del fluido (que generalmente variará con la distancia a la superficie sólida o pared) y el campo eléct rico debido a la carga superficai de la pared. El campo eléctrico está determinado por las cargas superficiales sobre el sólido y en el líquido, pero puede también incluirse un campo externo impuesto (como en el caso de electroforesis y electroósmosis). La relación entre el potencial en un punto y la distribución de carga está dado por la ecuación de Poisson. Las cargas en un fluido responden a tres fuerzas: i) Potencial eléctrico, ii) Potencial químico que tiende a regular las variaciones de concentración, y iii) Cuando se encuentra en movimiento, el líquido está sujeto en cada punto a fuerzas causadas por gradientes de 


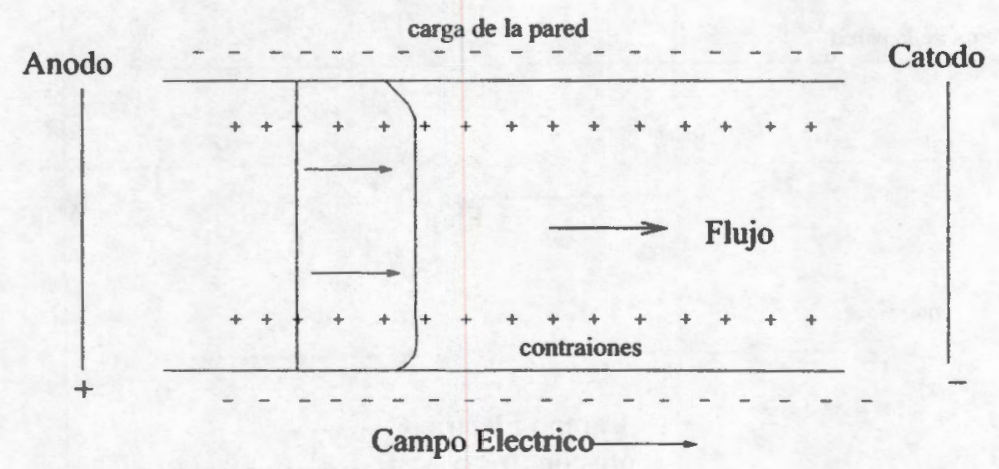

Flujo electroosmotico en un capilar

Figura 1.2:

presión y también a fuerzas laminares inducidas por partes vecinas de líquido moviéndose con diferentes velocidades.

Si un campo eléctrico es aplicado tangencialmente a lo largo de la superficie, se ejerce una fuerza sobre la superficie y sobre los iones de la doble capa eléctrica. La superficie cargada (más el material adherido a ella) tienden a moverse en la dirección impuesta por el campo eléctrico, mientras que los contraiones en la parte movible de la doble capa eléctrica muestran una migración neta en la dirección opuesta, llevando al solvente con ellos y causando un flujo de solvente.

Los fenómenos electrocinéticos tradicionales son cuatro: Electroósmosis, electroforesis, potencial de corriente (streaming potential) y potencial de sedimentación. En este trabajo nos concentraremos en el primero y tercero. La electroósmosis es el movimiento de la disolución electrolítica en relación a una superficie cargada (i.e. tubo capilar o medio poroso) debido a un campo eléctrico aplicado. La presión necesaria para contrarrestar el flujo electrostático es llamada presión electrostática. El flujo electroosmótico de un fluido típico en un capilar se muestra en la figura 1.2. Cuando el tubo capilar está cargado negativamente, el campo eléctrico aplicado ejerce una fuerza a lo largo de la dirección del cátodo sobre los iones positivos de la doble capa eléctrica, estos a su vez, arrastran la disolución electrolítica y el flujo aparece hacia el cátodo. El fenómeno de electroósmosis puede ser empleado para drenado de medios porosos y en la evaluación de superficies cargadas.

Potencial de corriente (streaming potential): En lugar de aplicar un campo eléctrico para mover un líquido a través de un capilar o poro, uno puede obligar al líquido a moverse aplicando un gradiente de presión, entonces la carga de la doble capa eléctrica que se forma cerca de la pared es llevada a través del capilar por el líquido, lo que da origen a una corriente eléctrica en el mismo sentido de la dirección del flujo del fluido (si las cargas son positivas). En la figura 1.3 una corriente es creada cuando una disolución electrolítica es bombeada a través de un capilar cargado negativamente. Por otro lado, existe una condición en la que se puede alcanzar el estado de corriente neta igual a cero al aplicar un potencial eléctrico en la dirección opuesta de la corriente. El potencial eléctrico que aplicado necesario para contrarestar la corriente inducida por el gradiente de presión es llamado potencial de 


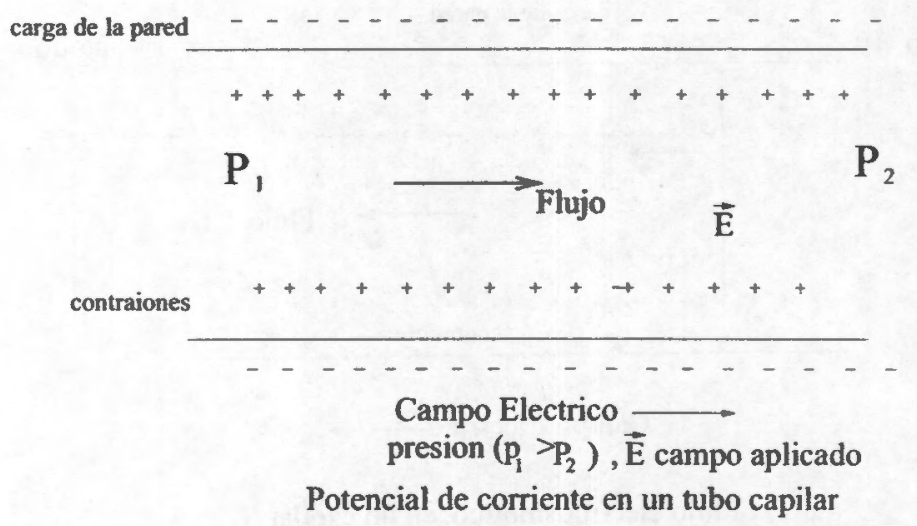

Figura 1.3:

corriente, y está relacionado con el gradiente de presión y con el potencial en la vecindad de la pared (potencial $\zeta$ ). En al figura 1.3 el campo eléctrico debido al potencial de corriente es de izquierda a derecha. Tenemos el objetivo de entender el flujo electroosmótico en un medio poroso o en tubos capilares de sección transversal de geometría variante, como por ejemplo. la situación a través de poros rectangulares y cilíndricos.

Dentro de algunas aplicaciones electrocinéticas, podemos citar el rechazo de sal en un medio poroso, tales como las arcillas, poros sólidos y membranas (por ej. celofán) que tienen la propiedad de rechazar la sal parcialmente cuando una disolución salina se filtra. En otras pálabras, la concentración de sal en el filtro es menor que en la disolución salina entrante de un poro o membrana. Para conservar el balance de masa de sal, el poro sólido actúa como un filtro que no permite que la sal pase a través de ella. El mecanismo físico del rechazo de sal en un capilar cargado es un tema del que nos ocuparemos en este trabajo. La carga superficial da origen a un perfil potencial que se extiende a una distancia comparable a la inversa de la longitud de Debye en la capilaridad del poro. Debe reconocerse que el rechazo de sal en un capilar es un fenómeno de ósmosis inversa donde el flujo de un líquido ocurre de un punto de alta a baja concentración de sal, generado por la influencia de un gradiente de presión impuesto.

Definiendo la conductividad $\sigma$ de una disolución como

$$
\mathbf{j}=-\sigma \nabla \psi
$$

donde $j$ es la densidad de corriente que resulta del flujo individual de todas las especies iónicas presentes en una disolución electrolítica cuando no hay gradiente de concentración y la disolución es eléctricamente neutra, $\psi$ es el potencial eléctrico, la ecuación anterior es una expresión de la ley de Ohm, válida sólo para las condiciones anteriores y no involucra ninguna aproximación en el tipo de disolución. La conductividad en una disolución depende de la naturaleza del soluto. La conductancia se define como $L=1 / R$ es decir el inverso de la resistencia $R$, en un electrolito al igual que en los metales, la resistencia entre 2 electrodos es directamente proporcional a la longitud que hay a través de la disolución entre los electrodos e inversamente proporcional a la sección transversal del poro (el área), $R=\frac{\rho l}{A}$, donde $\rho$ es 
una constante de proporcionalidad (resistencia específica o resistividad).

\subsubsection{Variables electrocinéticas}

Para entender el flujo electroosmótico en un medio poroso, presentaremos un flujo en un tubo recto con una carga en su superficie, ver [41] y [42]. La corriente total I de los iones es debida al flujo del fluido dentro del poro que es influenciado por un gradiente de presión y un campo eléctrico impuesto. En el caso de no tener una presión en el fluido en dirección a lo largo del eje paralelo a las paredes del poro, $p_{z}=0$, la razón del flujo volumétrico y la corriente son debidas al campo eléctrico aplicado, y la razón $(V / I)_{p_{z}=0}$, donde $V$ es el flujo volumétrico tiene una relación directa con el diámetro de los iones $a$, e inversamente proporcional a la conductividad de la disolución electrolítica. Cuando no hay flujo volumétrico neto $(V=0)$, la razón del gradiente de presión sobre el gradiente de potencial eléctrostático, $\frac{P_{z}}{E_{z}}$, siendo $P_{z}$ la presión osmótica y $E_{z}$ el gradiente de potencial aplicado, es una relación importante que será tratada en el siguiente capítulo. El potencial de corriente es el potencial electrostático asociado con la corriente que aparece a lo largo de un capilar en presencia de un gradiente de presión aplicado. Si $I=0$, la relación $\left(\frac{E_{z}}{p_{z}}\right)_{J=0}$, conecta la electroósmosis con el potencial de corriente y será analizado, en el siguiente capítulo.

\section{Efecto electroviscoso}

Cuando un líquido es forzado a pasar a través de un poro, influenciado por gradientes de presión y de potencial electrostático, y bajo la condición de corriente igual a cero, el flujo volumétrico puede ser calculado. El flujo volumétrico consta de dos términos, el primero es debido al gradiente de presión impuesto y el segundo término es debido al potencial de corriente.La reducción del flujo de volumen da la apariencia de un aumento de viscosidad y a ésto se le llama "efecto electroviscoso". Podemos identificar dos distintos efectos de la carga eléctrica para el caso de suspensiones coloidales reológicas, que llamaremos efectos electroviscosos [43]. El primero se origina de la distorsión de la parte difundida de la doble capa eléctrica debido al esfuerzo cortante, el segundo efecto es debido a las interacciones entre partículas que modifican las trayectorias $\mathbf{y}$ da origen a un aumento en el volumen excluido efectivo de las partículas. Para sistemas diluidos el primer efecto electroviscoso es más significativo que el segundo efecto, y para suspensiones coloidales no diluidas el segundo efecto es más significativo [44]. El primer efecto fue identificado primero por Von Smoluchowski debido a la interacción de la parte difundida de la doble capa eléctrica con el campo del flujo.

El segundo efecto electroviscoso es importante en el cual la probabilidad de interacción partícula-partícula aumenta. Aunque en sistemas no diluidos los dos efectos están presentes. El segundo efecto es dominante. cuando aumenta la concentración de partículas, por esta razón se considera que los cambios en el modelo de esfera dura a altas concentraciones es debido a efectos electroviscosos. 


\subsection{Cuerpos Sumergidos en un Electrolito}

\subsubsection{Conceptos Generales}

Un sistema formado por una mezcla homogénea, o aparentemente homogénea, de dos sustancias presenta en general una sustancia en mayor cantidad y otra en menor proporción que se encuentra dispersa en la primera. Entonces hablamos de una fase dispersora y una fase dispersa respectivamente. Las dispersiones se refieren a un estado de la materia que contiene otro cuerpo repartido uniformemente en su masa o también cuando algún componente de una mezcla se halla en mayor proporción que los demás. Se acostumbra clasificar las dispersiones como soluciones (o disoluciones < 1micra), los coloides o dispersión coloidal (1-100micras) y suspensiones ( $>100$ micras), son una clasificación en función del tamaño de las partículas de la fase dispersa.

Al componente que está en exceso se le denomina disolvente y al componente o componentes que se encuentran en menor proporción, soluto. En nuestro caso presentamos el estudio de una disolución electrolítica con una partícula cuya concentración tiende a cero, inmersa en la disolución, donde el soluto está formado por partículas de dos especies de igual tamaño, cargas opuestas iguales, y el disolvente (puede ser agua por ejemplo). Una característica de los sistemas coloidales es su gran superficie. Las partículas son tan pequeñas que la superficie efectiva de contacto de todas con la fase dispersora es enorme, en virtud de su gran área de contacto. Los sistemas coloidales se emplean frecuentemente en procesos de adsorción. fenómeno en el cual, moléculas o átomos de una sustancia se unen a la superficie de sólidos o líquidos. La ciencia de coloides abarca una amplia variedad de sistemas como cremas de afeitar, pinturas, cosméticos, cerveza, arcillas agrícolas, mayonesas o células biológicas: que tienen una característica en común que constan de pequeñas "partículas" de una sustancia distribuida más o menos uniformemente alrededor de otra; ésta última configuración o componente es continua mientras las "partículas" son discontinuas. Estas dos componentes del sistema pueden ser líquidos, gases o sólidos.

\subsection{Funciones de distribución}

Los est ados microscópicos (caracterizado por el conjunto de posiciones y momentos de las partículas $\left.\left\{\mathbf{r}_{1} \ldots \mathbf{r}_{N}, \mathbf{p}_{1} \ldots \mathbf{p}_{N}\right\}\right)$ de un sistema determinan, en gran parte, sus propiedades termodinámicas. Sin embargo, las cantidades macroscópicas medidas en el laboratorio son solamente cantidades promedio de los estados microscópicos del sistema. Con los métodos de la mecánica estadística de equilibrio podemos calcular la estructura ${ }^{1}$ de un fluido, que a su vez, nos sirve para calcular sus propiedades termodinámicas. A continuación vamos a resumir algunos resultados de la mecánica estadística de equilibrio(MEE), útiles para el estudio de los fluidos $|45,37,46|$.

En el estudio de fluidos por medio de la MEE, el concepto de función de distribución es de utilidad. Esta función mide la probabilidad de encontrar a las partículas del fluido en cierta configuración. En un sistema de partículas que interactúan vía un potencial dado, a T, V y N constantes (en el ensamble canónico), la probabilidad de encontrar a la partícula

\footnotetext{
${ }^{1}$ Este concepto será aclarado inás adelante
} 
1 en un elemento de volumen $d \mathbf{r}_{1}$ en $\mathbf{r}_{1}$, a la partícula 2 en un elemento de volumen $d \mathbf{r}_{2}$ en $\mathbf{r}_{2}$, etc., está dada por

$$
P\left(\mathbf{r}_{1} \ldots \mathbf{r}_{N}\right) d \mathbf{r}_{1} \ldots d \mathbf{r}_{N}=\frac{\exp \left\{-\beta U_{N}\left(\mathbf{r}_{1} \ldots \mathbf{r}_{N}\right)\right\}}{Z_{N}} d \mathbf{r}_{1} \ldots d \mathbf{r}_{N}
$$

donde $Z(N, V, T)=\int \ldots \int \exp \left\{-\beta U_{N}\left(\mathbf{r}_{1} \ldots \mathbf{r}_{N}\right)\right\} d \mathbf{r}_{1} \ldots d \mathbf{r}_{N}$ es la función de partición configuracional en el ensamble canónico y $U_{N}\left(\mathbf{r}^{N}\right)$ es la energía configuracional de las $N$ partículas. La densidad de probabilidad de encontrar a la partícula 1 en $\mathbf{r}_{1}, \ldots$ y a la partícula $\mathrm{n}$ en $\mathbf{r}_{n}$, independientemente de donde se encuentran las restantes, se encuentra integrando sobre las coordenadas de las partículas, desde $\mathrm{n}+1$ hasta $\mathrm{N}$

$$
P^{(n)}\left(r_{1} \ldots r_{n}\right)=\frac{\int \ldots \int \exp \left\{-\dot{\beta} U_{N}\left(r_{1} \ldots r_{N}\right)\right\} d r_{n+1} \ldots d r_{N}}{Z_{N}}
$$

Por otro lado, la densidad de probabilidad de encontrar a cualquier partícula en $\mathbf{r}_{1}, \ldots$, y a cualquier partícula en $\mathbf{r}_{n}$, independientemente del resto de las partículas está dada por

$$
\rho^{(n)}\left(\mathbf{r}_{1} \ldots \mathbf{r}_{n}\right)=\frac{N !}{(N-n) !} P^{(n)}\left(\mathbf{r}_{1} \ldots \mathbf{r}_{n}\right)
$$

La función $g^{(n)}\left(\mathbf{r}^{(n)}\right)$, definida como

$$
g^{(n)}\left(\mathbf{r}_{1} \ldots \mathbf{r}_{n}\right)=\frac{\rho^{(n)}\left(\mathbf{r}_{1} \ldots \mathbf{r}_{N}\right)}{\rho^{n}}
$$

es conocida como la función de correlación entre $n$ partículas y determina el grado de independencia, i. e., la correlación entre las partículas. Si las partícula de un sistema fueran independientes $g^{(n)}\left(\mathbf{r}^{(n)}\right)$ sería simplemente uno. Usando las Ecs. (1.4), (1.5) y (1.6), $g^{(n)}\left(\mathbf{r}^{(n)}\right)$ se escribe explícitamente como

$$
\begin{aligned}
g^{(n)}\left(\mathbf{r}_{1} \ldots \mathbf{r}_{n}\right) & =\frac{V^{n} N !}{N^{n}(N-n) !} \frac{\int \ldots \int \exp \left\{-\beta U_{N}\left(\mathbf{r}_{1} \ldots \mathbf{r}_{N}\right)\right\} d \mathbf{r}_{n+1} \ldots d \mathbf{r}_{N}}{Z_{N}} \\
& =V^{n}\left(1+O\left(N^{-1}\right)\right) \frac{\int \ldots \int \exp \left\{-\beta U_{N}\left(\mathbf{r}_{1} \ldots \mathbf{r}_{N}\right)\right\} d \mathbf{r}_{n+1} \ldots d \mathbf{r}_{N}}{Z_{N}}
\end{aligned}
$$

La función de correlación entre pares, $g^{(2)}\left(\mathbf{r}_{1}, \mathbf{r}_{2}\right)$, mide la densidad de probabilidad de encontrar a una partícula en $\mathbf{r}_{1} \mathbf{y}$ otra en $\mathbf{r}_{2}$, por lo que esta función proporciona la estructura promedio del sistema. Para el caso de partículas que interactúan vía potenciales esféricos, $g^{(2)}\left(\mathbf{r}_{1}, \mathbf{r}_{2}\right)$ depende sólo de la distancia entre las partículas, $r=\left|\mathbf{r}_{2}-\mathbf{r}_{1}\right|$, por lo que suele denotarse como $g(r)$. La cantidad $\rho g(r)$ mide la concentración local de partículas medida desde una partícula central, por lo que satisface

$$
\int_{0}^{\infty} \rho g(r) 4 \pi r^{2} d r=N-1 \approx N
$$


Suponiendo que las partículas del sistema interaccionan vía un potencial central aditivo por pares, $u(r)$, se pueden escribir las cantidades termodinámicas en función de $g(r)$. Por ejemplo, la energía interna se escribe de la siguiente manera

$$
E=\frac{3}{2} N k_{B} T+\frac{N}{2} \int_{0}^{\infty} \rho u(r) g(r) 4 \pi r^{2} d r
$$

De la misma forma puede escribirse una ecuación análoga para la presión

$$
p=\rho k T-\frac{\rho^{2}}{6} \int_{0}^{\infty} r \frac{d u(r)}{d r} g(r) 4 \pi r^{2} d r
$$

La función $g(r)$ es de particular importancia desde el punto de vista experimental ya que el factor de estructura, $S(k)$, se puede medir mediante experimentos de dispersión de luz o dispersión de partículas. La relación entre $S(k)$ y $g(r)$ está dada a través de la transformada de Fourier, i. e.,

$$
S(k)=\int g(r) e^{-i \mathbf{k} \cdot \mathbf{r}} d \mathbf{r}
$$

De esta forma, conociendo $S(k)$ podemos determinar $g(r)$ mediante la transformada inversa de Fourier.

Una forma conveniente de escribir $g^{(n)}\left(\mathbf{r}^{(n)}\right)$ es como

$$
g^{(n)}\left(\mathbf{r}_{1} \ldots \mathbf{r}_{n}\right)=\exp \left\{-\beta w^{(n)}\left(\mathbf{r}_{1} \ldots \mathbf{r}_{n}\right)\right\}
$$

donde la cantidad $w^{(n)}\left(\mathbf{r}^{(n)}\right)$ queda definida al sustituir ésta en la definición de $g^{(n)}$, Ec. (1.7), tomar el logaritmo de ambos lados, y tomar el gradiente respecto a las coordenadas de una de las $n$ partículas, lo cual nos da como resultado

$$
-\nabla w^{(n)}\left(\mathbf{r}_{1} \ldots \mathbf{r}_{n}\right)=\frac{\int \ldots \int\left(-\nabla_{j} U_{N}\left(\mathbf{r}_{1} \ldots \mathbf{r}_{n}\right)\right) \exp \left\{-\beta U_{N}\left(\mathbf{r}_{1} \ldots \mathbf{r}_{N}\right)\right\} d \mathbf{r}_{n+1} \ldots d \mathbf{r}_{N}}{\int \ldots \int \exp \left\{-\beta U_{N}\left(\mathbf{r}_{1} \ldots \mathbf{r}_{N}\right)\right\} d \mathbf{r}_{n+1} \ldots d \mathbf{r}_{N}}
$$

Como sabemos $-\nabla_{j} U_{N}\left(\mathbf{r}_{1} \ldots \mathbf{r}_{N}\right)$ es la fuerza que actúa sobre la partícula $j$ (con $1 \leq j \leq n$ ) para cualquier configuración de las $N-n$ partículas, por lo que la Ec. (1.10) nos representa la fuerza media $f_{j}^{(n)}$ sobre la partícula $j$, promediada sobre la configuración de todas las $n+1, \ldots, N$ partículas. Es decir $w^{(n)}$ es el potencial de la fuerza promedio actuando sobre la partícula $j$.

\subsubsection{Ensamble gran canónico}

La teoria de funciones de distribución es más poderosa si se desarrolla usando el ensamble gran canónico. A continuación presentamos un resumen de las propiedades de las funciones de distribución de un sistema clásico, abierto y de una sola componente. Dado que vamos a generalizar las ideas de la sección anterior a sistemas abiertos, las funciones de distribución [Ecs. (1.5) y (1.6)] se denotan como $\rho_{N}^{(n)}$ y $g_{N}^{(n)}$. Entonces, la probabilidad de encontrar $n$ 
partículas en los elementos de volumen $d \mathbf{r}_{1}, \ldots, d \mathbf{r}_{n}$ en $\mathbf{r}_{1}, \ldots, \mathbf{r}_{n}$, independientemente de $N$, es

$$
\rho^{(n)}=\sum_{N \geq n} \rho_{N}^{(n)} P_{N}
$$

donde $P_{N}$ es la probabilidad de que un sistema contenga a $N$ partículas, es decir

$$
P_{N}=\frac{e^{N \mu / k_{B} T} Q(N, V, T)}{\Xi(\mu, V, T)}=\frac{z^{N} Z_{N}}{N ! \Xi}
$$

donde

$$
\begin{aligned}
Z_{N} & =Z(N, V, T)=\int \ldots \int \exp \left\{-\beta U_{N}\left(\mathbf{r}^{N}\right)\right\} d \mathbf{r}_{1} \ldots d \mathbf{r}_{N} \\
z & =\frac{e^{\mu / k_{B} T}}{\Lambda^{3}} \\
\Xi & =\sum_{N \geq 0} \frac{z^{N} Z_{N}}{N !}
\end{aligned}
$$

$\operatorname{con} \Lambda=\left(\frac{2 \pi m k_{B} T}{h^{2}}\right)^{-1 / 2}$. De esta forma, usando las Ecs. (1.5) y (1.6) en la Ec. (1.11), se obtiene

$$
\rho^{(n)}\left(\mathbf{r}_{1}, \ldots, \mathbf{r}_{n}\right)=\frac{1}{\Xi}\left\{z^{n} e^{-\beta U_{n}}+\sum_{N=n+1}^{\infty} \frac{z^{N}}{(N-n) !} \int \ldots \int e^{-\beta U_{N}} d \mathbf{r}_{n+1} \ldots d \mathbf{r}_{N}\right\}
$$

donde $U_{j}$ denota la energía configuracional de un sistema de $j$ partículas. La definición dada en la Ec. (1.12) es el equivalente de la Ec. (1.5) para un sistema abierto. De la misma manera, la definición de la función de correlación entre $n$ partículas para un sistema abierto es igual a la de la Ec. (1.7). Por lo que ésta se escribe simplemente como

$$
g^{(n)}\left(\mathbf{r}_{1}, \ldots, \mathbf{r}_{n}\right)=\frac{V^{n}}{\bar{N}^{n} \Xi}\left\{z^{n} e^{-\beta U_{n}}+\sum_{N=n+1}^{\infty} \frac{z^{N}}{(N-n) !} \int \ldots \int e^{-\beta U_{N}} d \mathbf{r}_{n+1} \ldots d \mathbf{r}_{N}\right\}
$$

Las funciones de distribución en los ensambles canónico y gran canónico son las mismas para sistemas macroscópicos |47|, por lo que las Ecs. (1.8) y (1.9) se siguen cumpliendo, a menos que se involucren las fluctuaciones ${ }^{2}$.

Dada la definición de $g(r)$ en el ensamble gran canónico [Ec. (1.13)] se puede hacer un desarrollo en serie de ésta en potencias de $\rho$. Los primeros términos de este desarrollo son

$$
\begin{aligned}
g\left(\mathbf{r}_{1}, \mathbf{r}_{2}\right) & =e^{-\beta u\left(\mathbf{r}_{1} \cdot \mathbf{r}_{2}\right)}\left\{1+\rho \int f_{13} f_{23} d \mathbf{r}_{3}+\rho^{2} \int\left(f_{13} f_{34} f_{42}+2 f_{13} f_{34} f_{42} f_{32}+\right.\right. \\
& \left.\left.+\frac{1}{2} f_{13} f_{41} f_{32} f_{42}+\frac{1}{2} f_{13} f_{41} f_{34} f_{32} f_{41}\right) d \mathbf{r}_{3} d \mathbf{r}_{4}+\rho^{3} \int\left(f_{13} f_{34} f_{45} f_{52}+\ldots\right) d \mathbf{r}_{3} d \mathbf{r}_{4} d \mathbf{r}_{5}+\ldots\right\} .
\end{aligned}
$$

\footnotetext{
${ }^{2}$ Ver ref. [47]
} 
donde

$$
f_{i j} \equiv e^{-\beta u\left(\mathbf{r}_{i}, \mathbf{r}_{j}\right)}-1
$$

es conocida como la función de Mayer. El n-ésimo coeficiente es una suma de integrales de productos de la función de Mayer. Una integral de productos de las funciones de Mayer tiene una representación gráfica conocida como un diagrama de Mayer [45, 37, 40].

\subsection{La ecuación de Ornstein Zernike}

La función de correlación total se define como $h\left(\mathbf{r}_{1}, \mathbf{r}_{2}\right) \equiv g\left(\mathbf{r}_{1}, \mathbf{r}_{2}\right)-1$, para un fluido de una sola especie. En 1914 Ornstein [48] y Zernike [49] proponen dividir $h\left(\mathbf{r}_{1}, \mathbf{r}_{2}\right)$ en dos partes, una parte directa y una parte indirecta. La parte directa, dada por la función $c\left(\mathbf{r}_{1}, \mathbf{r}_{2}\right)$, llamada la función de correlación directa. La parte indirecta es la influencia propagada directamente de la partícula 1 a la 3 , la cual actúa sobre la partícula 2, directamente e indirectamente a través de otras partículas. Este efecto es pesado por la concentración y promediado sobre todas las posiciones de la partícula 3. Con esta descomposición de $h\left(\mathbf{r}_{1}, \mathbf{r}_{2}\right)$, se escribe la ecuación de Ornstein-Zernike.

$$
h\left(\mathbf{r}_{21}\right)=c\left(\mathbf{r}_{21}\right)+\rho \int h\left(\mathbf{r}_{23}\right) c\left(\mathbf{r}_{13}\right) d \mathbf{r}_{3},
$$

donde hemos supuesto que el potencial de interacción entre las partículas depende únicamnete de su posición relativa, $\mathbf{r}_{21}=\mathbf{r}_{2}-\mathbf{r}_{1}$, por lo que $h\left(\mathbf{r}_{1}, \mathbf{r}_{2}\right)=h\left(\mathbf{r}_{21}\right)$.

De la Ec. (1.10), la función $g\left(\mathbf{r}_{21}\right)$ está relacionada con el potencial de la fuerza media entre dos partículas, $w\left(\mathbf{r}_{21}\right)$, por

$$
g\left(\mathbf{r}_{21}\right)=\exp \left\{-\beta w\left(\mathbf{r}_{21}\right)\right\} .
$$

En un fluido multicomponente, la función de correlación total $\left(h_{i j}\left(\mathbf{r}_{21}\right) \equiv g_{i j}\left(\mathbf{r}_{21}\right)-1\right)$ entre dos partículas de las especies $i$ y $j$ en $\mathbf{r}_{1}$ y $\mathbf{r}_{2}$, respectivamente, están relacionadas con la función de correlación directa $c_{i j}\left(\mathbf{r}_{21}\right)$ por la ecuación de Ornstein-Zernike multicomponente, la cual, para un sistema de $k$ componentes está dada por

$$
h_{i j}\left(\mathbf{r}_{21}\right)=c_{i j}\left(\mathbf{r}_{21}\right)+\sum_{m=1}^{k} \rho_{m} \int h_{i m}\left(\mathbf{r}_{23}\right) c_{m j}\left(\mathbf{r}_{13}\right) d \mathbf{r}_{3},
$$

donde $\rho_{m}$ es la concentración de la especie $m$ y $\mathbf{r}_{21} \equiv \mathbf{r}_{1}-\mathbf{r}_{2}$ es la posición relativa entre las partículas 1 y 2. La ecuación de Ornstein-Zernike define la función de correlación directa. En esta ecuación tenemos dos funciones y ambas son desconocidas. Para poder resolver esta ecuación tenemos que emplear una teoría que nos dé una relación entre la función de correlación directa y la función de correlación total. Se han propuesto varias aproximaciones para la función de correlación directa fundamentadas en métodos de la teoría de líquidos. Entre estas aproximaciones tenemos las siguientes $[37,46]$

$$
\begin{gathered}
c_{i j}\left(\mathbf{r}_{21}\right)=-\beta u_{i j}\left(\mathbf{r}_{21}\right)+h_{i j}\left(\mathbf{r}_{21}\right)-\ln g_{i j}\left(\mathbf{r}_{21}\right), \\
c_{i j}\left(\mathbf{r}_{21}\right)=-\beta u_{i j}\left(\mathbf{r}_{21}\right),
\end{gathered}
$$




$$
c_{i j}\left(\mathbf{r}_{21}\right)=f_{i j}\left(\mathbf{r}_{21}\right) g_{i j}\left(\mathbf{r}_{21}\right) \exp \left\{\beta u_{i j}\left(\mathbf{r}_{21}\right)\right\},
$$

Las Ecs. (1.20), (1.21) y (1.22) se conocen como la aproximación de cadena hipertejida (HNC), la aproximación esférica media (MSA), y la aproximación de Percus-Yevick (PY), respectivamente. Siendo $u_{i j}\left(\mathbf{r}_{21}\right)$ es el potencial de interacción directa entre un par de partículas de las especies $i$ y $j$ y $f_{i j}\left(\mathbf{r}_{21}\right) \equiv \exp \left\{-\beta u_{i j}\left(\mathbf{r}_{21}\right)\right\}-1$, es la función de Mayer ${ }^{3}$. No es posible calcular $g\left(\mathbf{r}_{21}\right)$ de forma exacta ya que todas las teorías para calcularla ignoran varios tipos de diagramas. Por ejemplo, en la teoría HNC los diagramas puente son ignorados completamente, mientras que en la teoría de Percus-Yevick los diagramas puente y los diagramas producto no son considerados

\subsubsection{El modelo primitivo}

El modelo más simple que contiene los principales rasgos de un electrolito es el modelo primitivo (MP)|50|. Este modelo consiste en aproximar el disolvente por un medio continuo en el cual los iones se encuentran inmersos ${ }^{4}$. La interacción entre los iones se lleva a cabo vía un potencial, el cual, para dos iones con carga $q_{i}$ y $q_{j}$ separados por una distancia $r$, está dado por

$$
u_{i j}(r)=\left\{\begin{array}{cc}
\infty & r<\frac{a_{i}+a_{j}}{2} \\
\frac{q_{i} q_{j}}{\varepsilon r} & r \geq \frac{a_{i}+a_{j}}{2}
\end{array}\right.
$$

Donde $q_{i}=z_{i}$ e y $a_{i}$ son la carga y el diámetro del ion de la especie $i$, respectivamente; $z_{i}$ es el número de valencia y $\epsilon$ es la carga protónica. Es decir, estamos tratando a los iones como esferas duras cargadas. En el modelo primitivo restringido (MPR) todas las especies que forman al electrolito tienen el mismo tamaño. Para alcanzar el equilibrio termodinámico el fluido debe satisfacer la condición de electroneutralidad la cual se escribe de la siguiente forma

$$
\sum_{i=1}^{2} z_{i} \rho_{i}=0
$$

donde $\rho_{i}$ es la concentración de bulto de la especie $i$.

\subsubsection{Fluidos inhomogéneos: el método directo}

Un fluido influenciado por un campo externo. o un fluido que se encuentra en la vecindad de la superficie de una partícula coloidal, se acomoda de manera inhomogénea. En esta sección vamos a extender los métodos de la sección 1.4 al estudio de fluidos inhomogéneos. Los métodos de ecuaciones integrales ya han sido aplicados por Henderson et. al. al estudio de interfases $[51,52]$. Además de la propuesta de Kjellander et. al. [53\} donde también obtiene

\footnotetext{
${ }^{3}$ Esta definición difiere de la dada en la Ec. (1.16) donde $i$ y $j$ denotan posiciones, mientras que en este caso denotan especies.

${ }^{4}$ Este medio continuo tiene una constante dieléctrica $\varepsilon$, diferente de la constante dieléctrica del vacío.
} 
la función de distribución inhomogénea. Sin embargo, estos métodos solamente pueden ser empleados para fluidos en contacto con superficies planas.

Un método más general y con el cual pueden derivarse teorías para fluidos inhomogéneos fue propuesto por Lozada-Cassou [40,54], este método es conocido como el Método Directo (MD). Este método es más general que el método de Henderson, ya que puede emplearse para cualquier geometría. Por otro lado, con este método puede obtenerse como un caso particular el caso de Henderson. El método directo esta basado en el hecho fundamental de la equivalencia entre partículas y campos. Es decir, el hecho de que las partículas y campos se definen a través de su interacción con otras partículas o campos. Ya que en las teorías presentadas en la sección 1.4 no existe restricción en cuanto al tipo de partícula, el número de especies en el fluido y la concentración de cada especie; una fuente de campo externo puede ser considerado como una especie más del fluido, la cual se encuentra a dilución infinita. De esta forma, si llamamos $\alpha$ a la especie que representa a la fuente de campo externo, partiendo de la Ec. (1.19) para $n+1$ especies y tomando el caso límite de $\rho_{n+1}=\rho_{\alpha} \rightarrow 0$, la función de correlación entre la especie $\alpha$ y la especie $j$ está dada por:

$$
h_{\alpha j}\left(\mathbf{r}_{21}\right)=c_{\alpha j}\left(\mathbf{r}_{21}\right)+\sum_{m=1}^{n} \rho_{n n} \int h_{\alpha m n}\left(\mathbf{r}_{23}\right) c_{m j}\left(\mathbf{r}_{13}\right) d \mathbf{r}_{3},
$$

nótese que la suma no considera la especie $n+1$. Análogamente a la Ec. (1.18), la relación entre la función de distribución, $g_{a i}\left(\mathbf{r}_{21}\right)$, y el potencial de la fuerza promedio entre la partícula a y una partícula de la especie $i$ en $r_{21}$ viene dada por

$$
g_{\alpha i}\left(\mathbf{r}_{21}\right)=\exp \left\{-\beta w_{\alpha i}\left(\mathbf{r}_{21}\right)\right\}
$$

Para poder resolver la Ec. (1.25) es necesario conocer $c_{m j}\left(\mathbf{r}_{13}\right)$ y $c_{\alpha j}\left(\mathbf{r}_{21}\right)$. La primera $\left|c_{m j}\left(\mathbf{r}_{13}\right)\right|$ se obtiene de la solución de la ecuación de Orstein-Zernike para un fluido homogéneo de $n$ especies, como la Ec. (1.19). La segunda función $\left[c_{\alpha j}\left(\mathbf{r}_{21}\right)\right]$ puede aproximarse por cualquiera de las cerraduras PY, MSA ó HNC, las cuales tienen la siguiente forma funcional

$$
\begin{gathered}
c_{\alpha i}\left(\mathbf{r}_{21}\right)=f_{\alpha i}\left(\mathbf{r}_{21}\right) g_{\alpha i}\left(\mathbf{r}_{21}\right) \exp \left\{\beta u_{\alpha i}\left(\mathbf{r}_{21}\right)\right\} \\
c_{\alpha i}\left(\mathbf{r}_{21}\right)=-\beta u_{\alpha i}\left(\mathbf{r}_{21}\right), \\
c_{\alpha i}\left(\mathbf{r}_{21}\right)=h_{\alpha i}\left(\mathbf{r}_{21}\right)-\ln g_{\alpha i}\left(\mathbf{r}_{21}\right)-\beta u_{\alpha i}\left(\mathbf{r}_{21}\right) .
\end{gathered}
$$

Pueden construirse distintas teorías de líquidos dependiendo de la aproximación que se use para la función de correlación directa entre el campo externo y las partículas, y la función de correlación directa entre las partículas del bulto. La notación que se usa para denotar dichas teorías es la siguiente:

$$
\text { Siglas que denotan a } c_{a i} / \text { Siglas que denotan } \vec{a} c_{i j} \text {. }
$$

Ejemplo de las teorías que podemos emplear son: $\mathrm{HNC} / \mathrm{HNC}, \mathrm{PY} / \mathrm{HNC}, \mathrm{MSA} / \mathrm{HNC}$, etc. Este método puede emplearse en general para cualquier tipo de fluido, sin embargo, ha 
sido aplicado principalmente para el caso de fluidos cargados inhomogéneos, las cuales han resultado exitosas al compararse con los métodos de simulación molecular $[55,56,57]$.

Resumiendo lo anterior, tenemos que el concepto de fluido inhomogéneo corresponde a un fluido homogéneo que es afectado por un campo externo de cualquier tipo, este campo puede referirse también a superficies sólidas neutras o cargadas que introducen una alteración en la estructura original del fluido homogéneo, como por ejemplo partículas coloidales o macromoléculas, que ocupan lugares físicos que antes ocupaban las partículas del fluido homogéneo, y que obligan a los componentes del fluido homogéneo a acomodarse de una manera que llamamos inhomogénea. Es decir existe una equivalencia entre partícula y campo bien establecida que las identifica a través de la interacción de éstas con otras partículas o campos. Además, no existe restricción en el tipo de partícula, el número de especies que forman el fluido y la concentración de cada una de estas especies. Si el campo externo que afecta a nuestro fluido homogéneo puede ser considerado como una especie más de dilución infinita dentro del fluido, entonces tenemos un fluido inhomogéneo que puede ser estudiado de esta manera, a esta idea se le conoce como Método Directo [40, 26]. Este método es independiente de la geometría de la partícula que introduce la inhomogeneidad.

\subsubsection{La ecuación de Poisson-Boltzmann}

Cuando un electrolito se encuentra influenciado por un campo eléctrico el cual es producido por una superficie o por una partícula coloidal con carga, los contraiones (partículas cargadas del signo opuesto a la superficie) son atraídos hacia la superficie, mientras que los coiones (partículas cargadas del mismo signo a la superficie) tienden a ser repelidos. Debido a que el sistema se encuentra a $T>0$, i. e., las partículas del fluido tienen energía cinética, los contraiones no condensan totalmente sobre la superficie. Por otro lado, los coiones no son totalmente repelidos de la superficie debido a que no pueden disponer de todo el espacio accesible. Los contraiones y coiones se arreglan de tal forma que eventualmente el campo eléctrico es cancelado a cierta distancia de la superficie. Esta estructura es conocida como la doble capa eléctrica(DCE).

El tratamiento más simple para fluidos cargados se lleva mediante la ecuación clásica de Poisson-Boltzmann. En esta teoría, además de que solamente se consideran las interacciones de tipo electrostático, se emplea una aproximación sobre el potencial de la fuerza promedio de dos partículas, $w^{(2)}\left(\mathbf{r}_{12}\right)$ [Ec. (1.26)]. Consideremos una macropartícula inmersa en un electrolito en equilibrio, a la cual llamaremos la partícula a. De la electrostática sabemos que el potencial eléctrico depende de la distribución de carga como lo indica la ecuación de Poisson [58]

$$
\nabla^{2} \varphi(\mathbf{r})=-\frac{4 \pi}{\varepsilon} \rho_{e l}(\mathbf{r})
$$

donde $\rho_{e l}(\mathbf{r})$ es la densidad local de carga en $\mathbf{r}$ la cual está definida por

$$
\rho_{e l}(\mathbf{r})=\sum_{i=1}^{n} z_{i} e \rho_{i}(\mathbf{r})
$$

siendo $\rho_{i}(\mathbf{r})$ la concentración local de la especie $i$. Si calculamos el promedio de ensamble de 
ambos lados de la Ec. (1.30) tenemos como resultado

$$
\nabla^{2}\langle\varphi(\mathbf{r})\rangle=-\frac{4 \pi}{\varepsilon}\left\langle\rho_{e l}(\mathbf{r})\right\rangle=-\frac{4 \pi}{\varepsilon} \sum_{i=1}^{n} z_{i} e\left\langle\rho_{i}(\mathbf{r})\right\rangle
$$

donde los promedios de ensamble se indican con las cantidades entre corchetes. El promedio de la concentración local de la especie $i$ está relacionada con la función de distribución por

$$
\left\langle\rho_{i}(\mathbf{r})\right\rangle=\rho_{i} g_{\alpha i}(\mathbf{r}),
$$

donde $g_{\alpha i}(\mathbf{r})$, es la función de distribución de la especie $i$ alrededor de la especie $\alpha$ y $\rho_{i}$ es la concentración de bulto de la especie $i$. Usando la Ec. (1.33), la Ec. (1.32) se puede escribir como

$$
\nabla^{2}\langle\varphi(\mathbf{r})\rangle=-\frac{4 \pi}{\varepsilon} \sum_{i=1}^{n} z_{i} e \rho_{i} g_{\alpha i}(\mathbf{r})
$$

De la Ec. (1.26) sabemos que la función de distribución de la especie $i$ alrededor de $\alpha$ está relacionado con el potencial de la fuerza promedio sobre la partícula $i$ en $\mathbf{r}$. La teoría de Debye-Hückel supone que $[37,59]$

$$
w_{a i}(\mathbf{r})=z_{i} \epsilon\langle\varphi(\mathbf{r})\rangle
$$

por lo que

$$
g_{a i}=\exp \left\{z_{i} \epsilon\langle\varphi(\mathbf{r})\rangle\right\} .
$$

Si denotamos $\langle\varphi(\mathbf{r})\rangle=\psi(\mathbf{r})$, la Ec. (1.34) se escribe como

$$
\nabla^{2} \psi(\mathbf{r})=-\frac{4 \pi}{\varepsilon} \sum_{i=1}^{n} z_{i} e \rho_{i} \exp \left\{-\beta z_{i} e \psi(\mathbf{r})\right\}
$$

Esta ecuación diferencial es conocida como ecuación de Poisson-Boltzmann. De la electrostática sabemos que la solución de la ecuación de Poisson, Ec. (1.30), puede escribirse como

$$
\psi(\mathbf{r})=\psi_{\alpha}(\mathbf{r})+\frac{1}{\varepsilon} \int \frac{\rho_{c l}\left(\mathbf{r}_{1}\right) d \mathbf{r}_{1}}{\left|\mathbf{r}-\mathbf{r}_{1}\right|}
$$

donde $\psi_{a}(\mathbf{r})$ es la contribución al potencial debida a la partícula $a$, y el segundo término es la contribución al potencial debida a la distribución de carga del fluido. Haciendo uso de la Ecs. (1.35) y (1.38), obtenemos

$$
\begin{aligned}
g_{a i}(\mathbf{r}) & =\exp \left\{-\beta u_{a i}^{e l}(\mathbf{r})-\frac{z_{i} e \beta}{\varepsilon} \int \frac{\rho_{e l}\left(\mathbf{r}_{1}\right) d \mathbf{r}_{1}}{\left|\mathbf{r}-\mathbf{r}_{1}\right|}\right\} \\
& =\exp \left\{-\beta u_{a i}^{\epsilon l}(\mathbf{r})-\frac{z_{i} e^{2} \beta}{\varepsilon} \sum_{j=1}^{n} z_{j} \rho_{j} \int \frac{g_{a j}\left(\mathbf{r}_{1}\right) d \mathbf{r}_{1}}{\left|\mathbf{r}-\mathbf{r}_{1}\right|}\right\}
\end{aligned}
$$

en donde $u_{\alpha i}^{e l}(\mathbf{r})=z_{i} e \psi_{\mathrm{a}}(\mathbf{r})$. La cual es otra forma de escribir la ecuación de PoissonBoltzmann, o mejor dicho, esta es la versión integral de la ecuación de Poisson-Boltzmann. La ecuación de Poisson-Boltzmann ha sido la base de numerosos trabajos, p. ej., los trabajos 
de Gouy [60] y Chapman [61], en donde estudiaron una disolución electrolítica en presencia de un campo externo producido por una superficie plana cargada uniformemente. Las aproximaciones de las que hace uso la ecuación de Poisson-Boltzmann fueron examinadas rigurosamente por Kirkwood [62], quien demostró que la aproximación consiste en despreciar los términos de volumen excluido y de fluctuaciones. Aunque el análisis de Kirkwood fue hecho para electrolitos en bulto, éste puede ser extendido de manera directa para la doble capa eléctrica. La comparación entre los resultados de la ecuación de Poisson-Boltzmann con los de simulación molecular, muestra que la teoría falla cuando el volumen excluido no es despreciable o cuando las inetarcciones electrostáticas son muy fuertes [21].

\subsubsection{Modelo de Gouy-Chapman}

El modelo de Gouy-Chapman considera una superficie cargada inmersa en un electrolito que tiene un potencial electrostático constante en la superficie de $\psi_{s}$, dentro de la superficie se forma una capa de carga, los iones que compensan éstas cargas son considerados como cargas puntuales inmersas en un medio dieléctrico continuo. La atracción/repulsión acoplada con el movimiento browniano térmico aleatorio de los iones en el medio dieléctrico da origen a una capa eléctrica. Dentro de esta capa difundida, no hay neutralidad de carga. El equilibrio es establecido debido a las fuerzas atribuidas a la atracción/repulsión y difusión de los gradientes de concentración. El Modelo de Gouy-Chapman provee buenas predicciones cuantitativas para cuando el potencial en la superficie es bajo $(\sim 0.025 \mathrm{~V})$ y cuando la concentración del electrolito no es alta. El mayor defecto del modelo es que desprecia el tamaño finito de los iones (supone que los iones son cargas puntuales que pueden aproximarse a la superficie sin limite). Una modificación al modelo de Gouy-Chapman fue proporcionada por Stern (1924), conocida como el modelo de Gouy-Chapman modificado y éste supone que los iones puntuales sólo pueden aproximarse a la superficie cargada hasta una distancia que equivale al radio de un ión con tamaño finito.

\subsection{Fluidos en Equilibrio}

Un fluido puede estar dividido en diferentes regiones por medio de separaciones o fronteras llamadas interfases. Cada región se conoce como fase, las fases pueden ser iguales o diferentes. Si dos fases están en contacto y están interactuando entre si, pero están aisladas del exterior, habrá una transferencia de energía y materia, alcanzando el equilibrio, entre ellas, puede haber cambio de energía o materia localmente pero la energía total permanece constante. Cada fase debe tener la misma temperatura y si hay intercambio de partículas entre fases, cada una de estas debe tener la misma presión. Una característica importante es que el potencial químico es igual en las fases que están en contacto. Además, si estamos en un fluido cargado, la suma de la carga de todos los iones debe ser cero, si se transfiere materia de una fase a otra el fluido debe mantenerse electroneutro en el bulto.

Si tenemos dos fluidos cargados (electrolitos) separados por unä interfase permeable a todas las especies de ambas fases en equilibrio, la temperatura es igual en las dos fases y la presión puede no ser la misma cerca de la interfase. La condición del mismo potencial químico en ambas fases se sigue manteniendo y es importante. Expresando el potencial químico de 
los iones de la siguiente manera

$$
\mu_{i}=\mu_{i}^{0}+k T \ln a_{i}+z_{i} e \phi
$$

donde $\mu_{i}$ es el potencial químico de la partícula de especie $i, \mu_{i}^{0}$ es potencial químico de referencia de la especie $i, a_{i}$ es la actividad de la especie $i, z_{i}$ es la valencia de las partículas de especie $i, e$ es la carga elemental, $k$ es la constante de Boltmann, $T$ es la temperatura del fluido y $\phi$ es el potencial eléctrico del fluido. Considerando el sistema en equilibrio tal que el potencial químico para la especie $i$ es igual en las dos fases, llamadas fase $\alpha$ y fase $\beta$, tenemos

$$
\mu_{i}^{\alpha}=\mu_{i}^{\beta}
$$

para simplificar un poco las cosas, supongamos que tenemos sólo dos especies en el fluido que están al mismo potencial de referencia $\mu_{i}^{0}$ y es un electrolito simétrico donde $z_{1}=-z_{2}$, es decir $i=1,2$. Sustituyendo la ec.(1.40) en la ec.(1.41), tenemos dos ecuaciones siguientes

$$
\begin{aligned}
& \mu_{1}^{0 \alpha}+k T \ln a_{1}^{\alpha}+z_{1} e \phi_{1}^{\alpha}=\mu_{1}^{0 \beta}+k T \ln a_{1}^{\beta}+z_{1} e \phi_{1}^{\beta} \\
& \mu_{2}^{0 \alpha}+k T \ln a_{2}^{\alpha}+z_{2} e \phi_{2}^{\alpha}=\mu_{2}^{0 \beta}+k T \ln a_{2}^{\beta}+z_{2} e \phi_{2}^{\beta}
\end{aligned}
$$

donde $\mu_{i}^{0 \alpha}, \mu_{i}^{0 \beta}$ son los potenciales químicos de referencia en las dos fases, usando la condición de electrolito simétrico, sumando ambas ecuaciones y tomando $\Delta \phi=\phi_{i}^{\beta}-\phi_{i}^{\alpha}$ obtenemos

$$
a_{1}^{\alpha} a_{2}^{\alpha}=a_{1}^{\beta} a_{2}^{\beta}
$$

La actividad puede expresarse como $a_{i}=\gamma_{i} \rho_{i}$, donde $\gamma_{i}$ es el coeficiente de actividad de la especie $i$, y $\rho_{i}$ es la concentración de bulto de la especie $i, y$

$$
\ln \gamma=\frac{\rho}{k T} \int_{0}^{1} \int_{0}^{\infty} u(x) g(x, \xi) d x d \xi
$$

donde $\xi$ es un parámetro de acoplamiento, ver $([47])$. Aplicando condición de electroneutralidad total del sistema

$$
z_{1} \rho_{1}^{\alpha}+z_{2} \rho_{2}^{\alpha}+z_{1} \rho_{1}^{\beta}+z_{2} \rho_{2}^{\beta}=0
$$

entonces si $z_{1}=-z_{2}$

$$
\rho_{1}^{\alpha}-\rho_{2}^{\alpha}=\rho_{1}^{\beta}-\rho_{2}^{\beta}
$$

o

$$
\rho_{1}^{\alpha}-\rho_{1}^{\beta}=\rho_{2}^{\alpha}-\rho_{2}^{\beta}
$$

de la relación $(1.43)$

$$
\rho_{1}^{\alpha} \gamma_{1}^{a} \rho_{2}^{a} \gamma_{2}^{a}=\rho_{1}^{\beta} \gamma_{1}^{\beta} \rho_{2}^{\beta} \gamma_{2}^{\beta}
$$

en todo el espacio, observamos que para una interfase plana permeable el problema es simétrico para ambos lados de la interfase y entonces las integrales que relacionan la ec.(1.44), implican $\gamma_{1}^{\alpha}=\gamma_{1}^{\beta}$ y $\gamma_{2}^{\alpha}=\gamma_{2}^{\beta}$, entonces 


$$
\rho_{1}^{\alpha} \rho_{2}^{\alpha}=\rho_{1}^{\beta} \rho_{2}^{\beta}
$$

pero en el bulto $\rho_{1}^{a}=\rho_{1}^{\beta}$ y $\rho_{2}^{a}=\rho_{2}^{\beta}$, por la misma simetría del problema, calculando la presión osmótica como la diferencia de presiones de ambas fases $P=\Pi_{\beta}-\Pi_{\alpha}$,

$$
P=k T \rho=k T\left(\rho_{1}^{\beta}+\rho_{2}^{\beta}-\left(\rho_{1}^{\alpha}+\rho_{2}^{\alpha}\right)\right)
$$

y ésto es cero. Analizando la ec.(1.43) para el caso de baja concentración, en este límite $\gamma_{i} \rightarrow 1$ y también $a_{i} \rightarrow \rho_{i}$, entonces

$$
\rho_{1}^{\alpha} \rho_{2}^{\alpha}=\rho_{1}^{\beta} \rho_{2}^{\beta}
$$

la electroneutralidad de ambas fases en el bulto requiere que $z_{1} \rho_{1}^{\alpha}+z_{2} \rho_{2}^{\alpha}=0, \quad$ y $z_{1} \rho_{1}^{\beta}+$ $z_{2} \rho_{2}^{\beta}=0$, entonces $\rho_{1}^{\alpha}=\rho_{2}^{\alpha}$ y $\rho_{1}^{\beta}=\rho_{2}^{\beta}$. La presión osmótica está dada como la diferencia de presiones en el bulto de ambas fases, considerando que las componentes están diluidas, la presión osmótica es

$$
P=k T \rho=k T\left(\rho_{1}^{\beta}+\rho_{2}^{\beta}-\left(\rho_{1}^{\alpha}+\rho_{2}^{\alpha}\right)\right)
$$

para que la anterior ecuación sea igual a cero las densidades de bulto en ambas fases deben ser iguales, y esto va de acuerdo con (1.45), cuando la concentración de los iones es suficientemente baja, entonces la presión osmótica es cero en el bulto. Para el caso de una sola placa encontramos que la presión osmótica es siempre cero.

Si tenemos un sistema con dos líquidos iguales $\alpha$ y $\beta$ separados por una pared rígida (una membrana por ejemplo) donde la pared se mantiene fija de alguna forma, tal que las presiones de un lado y del otro de la pared pueden ser diferentes. Decimos que tenemos tres restricciones: no existe intercambio de energía con el exterior, la densidad total de partículas es constante y una forma que impide que se mueva la pared e implica la existencia de una diferencia de presiones $P_{\alpha}-P_{\beta}$ (presión osmótica). Llamaremos a la concentración de un líquido $c_{1}$ y del otro líquido $c_{2}$. El sistema alcanza su estado de equilibrio. Podemos decir que el sistema tiene 4 restricciones adicionales, una térmica que implica $T=c t e$, una geométrica que indica que el volumen es constante, y dos que se pueden llamar "químicas" dadas a través de la membrana que restringen $c_{1}$ y $c_{2}$ y también que $P_{\alpha} \neq P_{\beta}$, cuando $P_{\alpha}=P_{\beta}$ indica que hemos modificado alguna otra restricción. Si removemos una restricción del sistema se induce un cambio de estado, éste es un proceso (llamado proceso inducido) que al finalizar deja al sistema en un estado de equilibrio en el que está menos restringido. Los potenciales químicos en ambas regiones es una característica del equilibrio químico.

\subsection{Fuerza entre dos cuerpos cargados}

Cuando dos superficies cargadas (o dos partículas coloidales) con igual carga, inmersas en un electrolito y forman una doble capa eléctrica difundida cada una se acercan entre sí, sus respectivas doble capas se empiezan a superponer; entonces las distribuciones de potencial eléctrico y de iones cerca de una partícula en la vecindad de otra partícula ya no son simétricas con respecto al punto medio entre las dos superficies, hasta que alcanzan el equilibrio. Esto 


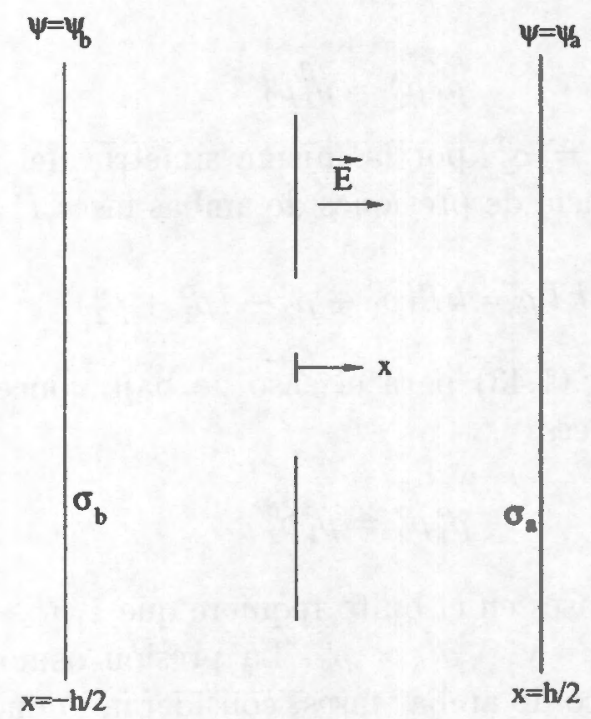

Figura 1.4: Geometría de placas paralelas

causa esfuerzos asimétricos de origen eléctrico sobre la superficie de las partículas coloidales y como resultado, éstas experimentan una fuerza. Considerense dos placas planas separadas a una distancia fija y a potenciales $\psi_{a}$ y $\psi_{b}$ respectivamente sobre las superfices de las placas. Estos potenciales son constantes e iguales (se pueden tener también cargas iguales sobre las superficies), ver fig(1.4). La ecuación de Poisson se puede escribir como

$$
\frac{d^{2} \psi}{d x^{2}}=-\frac{4 \pi \rho_{e l}}{\epsilon}
$$

donde $\psi$ es el potencial eléctrico, $\rho_{e l}$, es la densidad de carga libre. El fluido se encuentra en estado estacionario, con una velocidad promedio de los iones igual a cero o diferente de cero. Para los casos anteriores podriamos tener la concentración de los iones independientes del tiempo, lo cual sería una configuración de equilibrio.

La razón de cambio de momento es igual a la fuerza

$$
\frac{d(m \mathbf{v})}{d t}=\mathbf{F}
$$

y la a razón del cambio total de momento por unidad de volumen es igual a la suma de todas las fuerzas actuando por unidad de volumen es

$$
\frac{d(\rho \mathbf{v})}{d t}=\rho \mathbf{F} / \mathbf{m}
$$

La fuerza está compuesta de contribuciones que incluyen fuerzas externas (como la gravedad), fuerzas de presión y fuerzas viscosas, que podemos escribir como

$$
\mathbf{F}=\mathbf{F}_{\text {ext }}+\mathbf{F}_{\text {pres }}+\mathbf{F}_{\text {vis }}
$$


donde $\mathbf{F}$ es la fuerza neta por unidad de volumen. $\mathbf{F}_{\text {ext }}$ es la fuerza externa en el fluido, $\mathbf{F}_{\text {pres }}$, es la fuerza debido a la presión del fluido y $\mathbf{F}_{\text {vis }}$ es la fuerza debido a la viscosidad. La densidad es constante, para el caso de un fluido incompresible.

La ecuación de momento por unidad de volumen $p_{0}$ con relación con la fuerza eléctrica es

$$
\rho_{e l} \mathbf{E}=\nabla p_{0}
$$

donde $\nabla p_{0}$ es la fuerza por unidad de volumen. Esta ecuación se puede deducir directamente del equilibrio de fuerzas, en un estado estacionario, donde la suma de fuerzas es igual a cero es

$$
\sum_{i} F_{i}=0
$$

A partir de a ecuación (1.53), que se puede escribir de una manera conocida como la ecuación de Navier-stokes

$$
\rho \frac{\partial \mathbf{v}}{\partial t}+\rho \mathbf{v} \cdot \nabla \mathbf{v}=-\nabla P+\eta \nabla^{2} \mathbf{v}+\left(\rho \mathbf{g}+\mathbf{F}_{\mathbf{E}}\right)
$$

donde los términos de la izquierda corresponden a $\mathbf{F}$ y el primer término de la derecha es $\mathbf{F}_{\text {pres }}$, el segundo término es $\mathbf{F}_{\text {vis }} \mathbf{y}$ el tercer término es $\mathbf{F}_{\text {ext }}$,

$v$ es el vector velocidad del fluido.

$\rho$ es la concentración del fluido.

$P$ es la presión del fluido.

$\mathrm{g}$ es el vector aceleración debido a la gravedad.

$\mathbf{F}_{\mathbf{E}}$ es la fuerza eléctrica del campo creado por las cargas o el potencial de las placas.

$t$ es el tiempo.

$\eta$ es la viscosidad del fluido.

$\nabla$ es el operador gradiente.

$\nabla^{2}$ es el operador Laplaciano.

cada término en la ecuación anterior representa una fuerza por unidad de volumen. De izquierda a derecha el primer término de la izquierda relaciona el cambio de la velocidad del fluido con el tiempo en un lugar dado. Para condiciones de estado estacionario, este término es cero, el segundo término de la izquierda es debido a la inercia del fluido, éste es despreciable a bajas velocidades del fluido y también es cero en el caso estacionario de velocidad cero. El tercer término en el lado derecho del signo igual es debido a la presión, el cuarto término es debido a la viscosidad del fluido (esfuerzo cortante), y el último término es debido a la gravedad y al campo eléctrico externo.

Cuando un fluido es un electrolito, es de interés el movimiento de los aniones y cationes, asi como el movimiento del fluido en el bulto, para este fin la transferencia de masa en una solución electrolítica requiere de una descripción del movimiento de las especies iónicas móviles, balance de material, flujo de corriente, electroneutralidad total y mecánica de fluidos.

En la ecuación anterior la fuerza debido al campo eléctrico es una fuerza externa $F_{E}$, la fuerza eléctrica es considerada una fuerza que actúa sobre el cuerpo de las partículas, como lo es la gravedad. En general la fuerza eléctrica por unidad de volumen del fluido para una constante dieléctrica variable esta dada por 


$$
\mathbf{F}_{E}=\rho_{e l} \mathbf{E}-\frac{1}{2} \epsilon_{0} \mathbf{E} \cdot \mathbf{E} \nabla \epsilon+\frac{1}{2} \epsilon_{0} \nabla\left[\left(\frac{\rho \partial \epsilon}{\partial \rho}\right)_{T} \mathbf{E} \cdot \mathbf{E}\right]
$$

$\rho$ es la densidad de la disolución que en un electrolito puede coincidir con $\rho_{\ell l}$ que es la densidad de carga libre $([3,63]$. Consideramos que $\epsilon=c t e$. Entonces la fuerza por unidad de volumen de fluido aparece en función del campo eléctrico $\mathbf{F}_{E}=\rho_{e l} \mathbf{E}$.

Introduciendo la fuerza eléctrica en la ecuación de Navier-Stokes (la ec. 1.56),

$$
\rho \frac{\partial \mathbf{v}}{\partial t}+\rho \mathbf{v} \cdot \nabla \mathbf{v}=-\nabla P+\eta \nabla^{2} \mathbf{v}+\rho \mathbf{g}+\rho_{e l} \mathbf{E}
$$

Para el caso estacionario en un punto, la velocidad promedio es constante $(\mathbf{v}=\mathbf{c t e})$,

$$
\nabla P=\eta \nabla^{2} \mathbf{v}+\rho \mathbf{g}+\rho_{e l} \mathbf{E}
$$

y despreciando el efecto de la gravedad, entonces

$$
\nabla P=\eta \nabla^{2} \mathbf{v}+\rho_{e l} \mathbf{E}
$$

Esta ecuación la podemos descomponer en tres ecuaciones, para las tres coordenadas. Si tenemos un desplazamiento del fluido en dirección paralela a las placas (eje z) por la aplicación de un gradiente de presión $P_{z}$ y un gradiente de potencial eléctrico $E_{z}$, entonces la ecuación de Navier-Stokes para un flujo laminar en estado estacionario entre las placas, es

$$
P_{z}+\eta \frac{d^{2} v(x)}{d x^{2}}+\rho_{e l} E_{z}=0
$$

donde $v(x)$ es la velocidad del fluido en dirección $z$.

Para el caso particular de que no haya variación de la velocidad con respecto a las coordenadas, en la ecuación (1.59) el primer término de la derecha de la ecuación anterior es cero.

$$
\nabla P=\rho_{e l} \mathbf{E}
$$

Para la dirección perpendicular a las placas (dirección x), la ecuación (1.60) la podemos escribir como

$$
\frac{d P}{d x}=\rho_{e l} E_{x}
$$

por otro lado, sabemos que $\int_{V} \rho_{\epsilon l} d V=q$ y que $E=$ cte, para la placa plana, integrando la ecuación 1.61 , tenemos

$$
\begin{gathered}
\int_{V} \frac{d P}{d x} d V=\int_{V} \rho_{e l} E_{x} d V \\
P A=q E_{x}
\end{gathered}
$$

$A$ es el área, entonces $F_{x}=P A$. 
$F_{x}$ es la fuerza que actúa sobre el fluido en dirección $\mathrm{x}$ y $E_{x}$ es el campo eléctrico total producido por las paredes de las placas. Tomando la expresión $E_{x}=-\frac{d \psi}{d x}$ donde $\psi$ es el potencial eléctrico, y sustituyendola en la Ec. (1.61), combinando esta última ecuación con la ec. (1.50), tenemos que

$$
\frac{d P}{d x}-\frac{\epsilon}{4 \pi} \frac{d^{2} \psi}{d x^{2}} \frac{d \psi}{d x}=0
$$

(ver apéndice E). La ecuación anterior relaciona la presión (presión osmótica) con el potencial $\psi$, la presión se origina debido a la variación de la concentración iónica entre las placas y la disolución electrolítica en los alrededores, integrando la ec. (1.64)

$$
P-\frac{\epsilon}{8 \pi}\left(\frac{d \psi}{d x}\right)^{2}=C_{1}
$$

$C_{1}$ es una constante de integración, en este caso es la fuerza requerida por unidad de área sobre las placas. Para este caso particular, resulta que la diferencia entre la presión $p$ y la fuerza relacionada con el potencial eléctrico al cuadrado por unidad de área es constante en cualquier lugar entre las dos placas. Para evaluar esta fuerza necesitamos calcular $p$ y $\psi$.

\subsubsection{La carga Inducida en un Fluido}

Cuando un cuerpo cargado llamado electrodo (particularmente consideremos que tiene la forma de una placa plana cargada infinita de ancho finito $d$ se encuentra inmerso en un fluido se crea una inhomogeneidad en éste debido a dos factores principalmente: 1) El volumen excluido (u ocupado) por la placa. 2) El campo eléctrico producido por la carga de la placa. Esta inhomogeneidad induce una carga en el fluido manteniéndose neutro todo el sistema (condición de electroneutralidad), debiendose cumplir que la suma de la carga inducida en el fluido más la carga del electrodo debe ser igual a cero [8]; es decir,

$$
q_{p a r e d}=-\int_{V} \rho_{\epsilon l}(\mathbf{r}) d \mathbf{r}
$$

donde $q_{\text {purted }}$ es la carga sobre la superficie del electrodo y la integral es la carga inducida por la carga de la superficie, $\rho_{\mathrm{el}}$ es la densidad de carga volumétrica inducida en el fluido, definido como $\rho_{e l}=\sum_{i=1}^{N} \rho_{i} z_{i} e$, tal que $\rho_{i}$ es la densidad de partículas por unidad de volumen de especie $i$ y $z_{i}$ es la valencia de la especie $i$.

En nuestro sistema la carga la tenemos localizada sobre la superficie de la placa. Podemos expresar ésta en forma de la densidad carga superficial y también en el fluido, así

$$
\sigma_{1}+\sigma_{2}=-\int_{-\infty}^{x_{\alpha}} \rho_{\epsilon l}(x) d x-\int_{x_{B}}^{\infty} \rho_{e l}(x) d x
$$

la primera integral de la ecuación representa la carga inducida en el fluido en la parte izquierda que llamaremos región $\alpha$, observese la figura 1.5, y la segunda integral es la carga inducida del otro lado llamada región $\beta$, definimos estas integrales, de la siguiente manera

$$
\sigma_{\alpha} \equiv \int_{-\infty}^{x_{\alpha}} \rho_{e l}(x) d x
$$




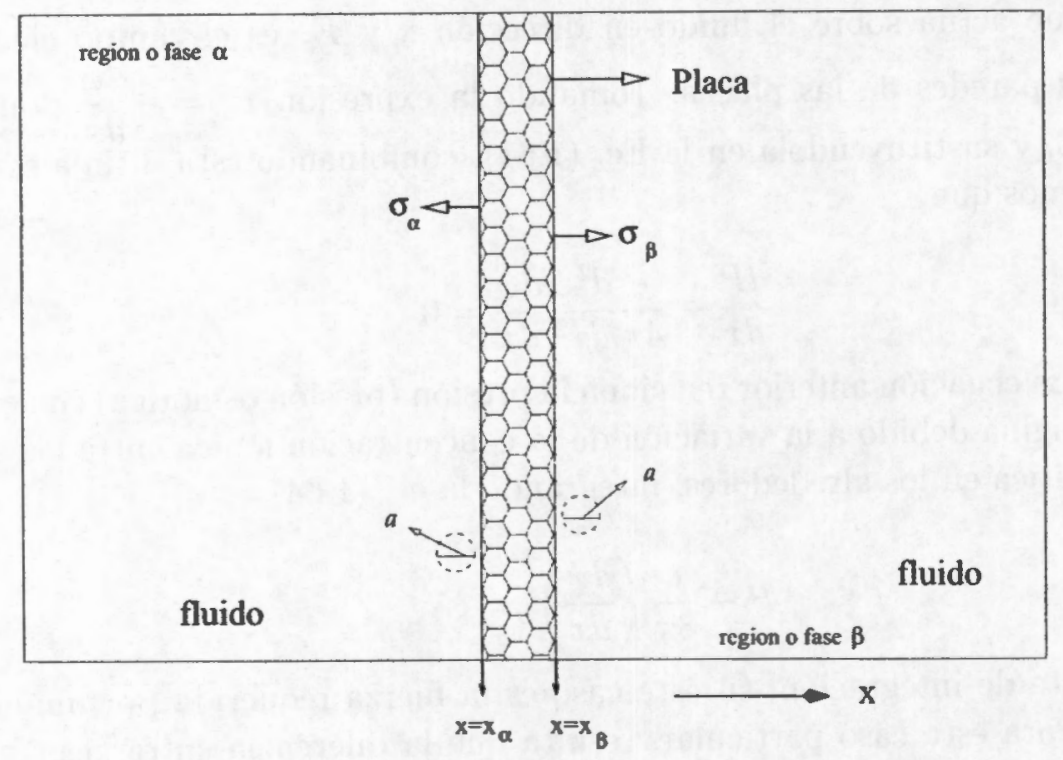

Figura 1.5: modelo de dos fases separadas por una pared

y

$$
\sigma_{\beta} \equiv \int_{x_{\beta}}^{\infty} \rho_{e l}(x) d x
$$

donde $x_{\alpha}$ y $x_{\beta}$ corresponden a los puntos sobre las superficies de las paredes de la placa.

Para el caso de dos placas, tres, cuatro, etc. inmersas en un electrolito las partículas se distribuyen de manera similar como en la fig. $1.6 \mathrm{~b}$ que representa cómo se difunden las partículas en dos placas.

Helmholtz fue el primero que desarrolló un modelo de la doble capa eléctrica e introdujo este concepto en 1850, para la carga sobre una superficie metálica en una disolución líquida. Esta carga era balanceada por una carga igual en la región líquida, él imaginó un arreglo de cargas en dos planos paralelos, ver fig. 1.6a, formando una especie de "condensador molecular". Sin embargo, el movimiento térmico hace que los contraiones se extiendan en el espacio formando una doble capa difundida (ver fig. 1.6b), subsecuentemente se observó que el movimiento térmico de las cargas en el medio dispersor, distribuía las cargas de alguna manera sobre la región, especialmente cerca de la superficie cargada. El modelo de la doble capa en estas circunstancias es el modelo de Gouy-Chapman donde además se consideran los iones como cargas puntuales y el material de la pared tiene la misma constante dieléctrica que el fluido electrolítico. 


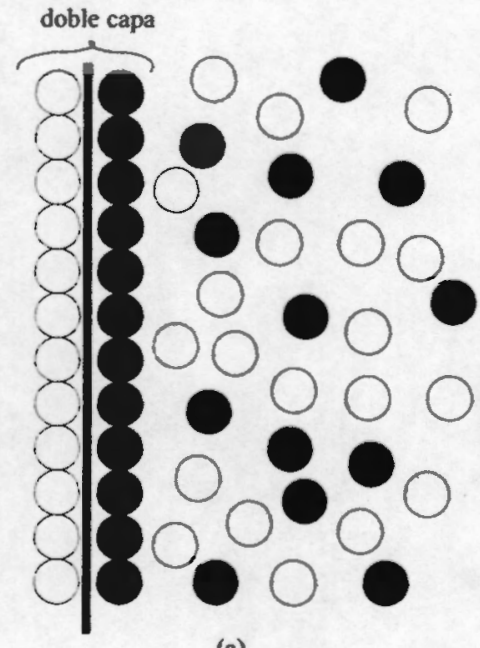

(a)

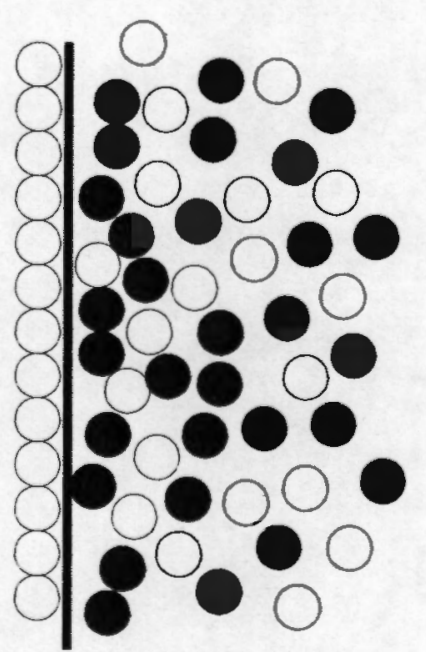

(b)

carga positiva

carga negativa

Figura 1.6: La doble capa eléctrica. (a) De acuerdo al modelo de Helmholtz, (b) La doble capa eléctrica resultante del movimiento térmico. 
24 


\section{Capítulo 2}

\section{Efecto de la geometría en las propiedades electrocinéticas de electrolitos confinados en poros de forma cilíndrica o de rendija plana.}

\subsection{Introducción}

Un fluido iónico (electrolito) confinado dentro de un poro cuyas paredes internas están cargadas, muestran importantes fenómenos de transporte, que a la vez juegan un papel muy destacado en física de líquidos y soluciones en medios porosos [64], incluyendo películas comprimidas superficialmente [65], o sustancias atrapadas en poros, como zeolitas [66], minerales arenosos [67], nanotubos de carbón [68], membranas [69], etc. Cuando una presión y/o un gradiente de potencial eléctrico son aplicados a través de un material poroso, que contiene una solución iónica, un flujo volumétrico neto y una corriente eléctrica neta son establecidas, dando origen a la conductancia capilar, el potencial de corriente y a la retardación electroviscosa; a estos fenómenos, se les conocen, como fenómenos electrocinéticos [70, 71].

Estos efectos pueden estar acompañados de otros como por ejemplo, rechazo de sales por un poro (exclusión de iones) |70|. que son de gran interés en el campo de dispersiones coloidales $[70,72]$. La hidrodinámica líneal establece que la correlación entre flujos y fuerzas es a primer orden lineal. Es decir, si estas fuerzas son pequeñas (presión y/o gradiente de potencial), el flujo volumétrico y la corriente eléctrica están acopladas linealmente a través de un conjunto de ecuaciones cuyos coeficientes son llamados coeficientes de transporte de Onsager, $L_{m n}$. Por tanto, considerando un flujo en estado estacionario y laminar dentro de un poro, las expresiones de los coeficientes de transporte de Onsager pueden derivarse en términos del potencial electrostático medio y de los perfiles de concentración iónicos.

Las propiedades electrocinéticas de un electrolito confinado por un poro de rendija de placas planas ha sido estudiada anteriormente en los trabajos de $[73,74,75]$, para los poros cilíndricos en $[41,76,42]$, y para materiales porosos en $\mid 77,78]$; estos estudios se basan en la aproximación de Poisson-Boltzmann (PB), para obtener los perfiles de concentración iónica 
y el potencial electrostático medio dentro del poro. En esta teoría no se toma en cuenta el tamaño de los iones, pero es una aproximación importante, debido a su simplicidad y relativa exactitud bajo ciertas condiciones; como son: muy baja concentración del electrolito y baja densidad de carga superficial. $\left(0,01 \mathrm{M}, 0,01 \mathrm{C} / \mathrm{m}^{2}\right)$ La aproximación esférica media de cadena hipertejida (HNC/MSA) ha sido usada exitosamente para calcular la estructura de un electrolito confinado en un modelo primitivo restringido (RPM) $[21,79,80,81,82]$. Asimismo, este modelo ha sido aplicado al estudio de los fenómenos electrocinéticos en un electrolito con RPM, confinado por un poro de rendija plana $[9,10,83]$. Esta teoría tiene ventajas sobre la teoría de $\mathrm{PB}$ ya que incorpora el tamaño de iones, correlaciones de corto alcance de los iones y en general muestra buena concordancia con cálculos de simulación bajo ciertas condiciones de confinamiento, concentración del electrolito y valencia del mismo $[84,57,56]$. En el caso de un poro de geometría cilíndrica, las propiedades electrocinéticas, han sido estudiadas en varios artículos, en el trabajo pionero de Rice y Whitehead [41] se usó la ecuación de PB linearizada (Debye-Hückel) para obtener el potencial electrostático medio. Levine et al. [42], extendió los resultados usando la ecuación no lineal de PB. Citando más estudios tenemos que descripciones de la estructura de un electrolito confinado en un poro cilíndrico, fueron dadas a través de soluciones aproximadas a la ecuación de $\mathrm{PB}[85,86,87,88]$ y por simulaciones de Monte Carlo [89, 90]. De la misma manera estudios teóricos con aproximaciones de PB, que incorporan correlaciones de corto alcance iónico han sido dadas a través de teorías de ecuaciones integrales $[89,81,82]$.

Una descripción teórica de la estructura de la doble capa eléctrica de un electrolito confinado dentro de un cilindro usando $\mathrm{HNC} / \mathrm{MSA}$ fue reportado en [81], junto con el método directo (MD) $[91,26,40]$. El efecto de la curvatura que encierran las superficies de los poros en las propiedades electrocinéticas de un fluido iónico es de interés particular en ciencia de coloides donde, frecuentemente mediciones hechas sobre superficies cóncavas o curvas son interpretadas usando resultados de poros de rendija plana [2]. Se pudo observar que estudios teóricos de los fenómenos, electrocinéticos considerando efectos de curvatura y tamaño de iones aún no han sido reportados. En este trabajo desarrollamos los cálculos de los perfiles del potencial electrostático medio y de concentración, los coeficientes de actividad media y las propiedades electrocinéticas para un electrolito simétrico confinado en poros cilindrico y de rendija plana. Los perfiles de concentración reducidos se obtuvieron por medio de las teorías de $\mathrm{PB}$ y $\mathrm{HNC} / \mathrm{MSA}$, que a su vez son usados en las ecuaciones lineales hidrodinámicas para calcular las propiedades electrocinéticas. Podemos observar una comparación de los resultados de ambas teorías, y de las dos geometrías, plana y cilíndrica. En la sección 2 presentaremos una descripción breve de los modelos teóricos de los poros cilíndrico y de rendija plana. Además, derivamos las ecuaciones integrales de la teoría HNC/MSA y de la teoría de Posson-boltzmann, para las dos geometrías. Se analizan los poros de tamaño infinito y los casos límites de iones puntuales. También se deriva can detalle las ecuaciones electrocinéticas para el poro cilíndrico; y para el caso del poro de rendija plana presentamos una síntesis de la derivación de las ecuaciones electrocinéticas pues ya están deducidas en la referencia $[9,10,83]$. A continuación, en la sección 3 , discutimos los resultados y finalmente en la sección 4 presentamos las conclusiones para este capítulo. 


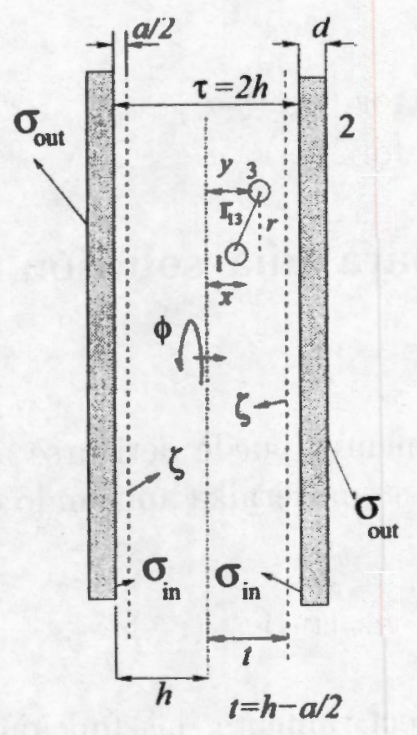

(a)

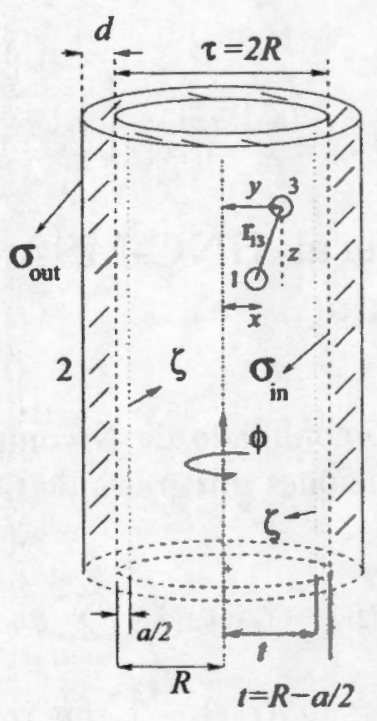

(b)

Figura 2.1: Modelos geométricos (a) poro de rendija plana y (b) poro cilíndrico.

\subsection{La teoría y los modelos}

Los sistemas que estudiaremos son i) un poro de rendija plana, formado de dos placas infinitas paralelas, cargadas, con una distancia de separación entre las superficies interiores de $\tau=2 h$ (ver Fig. 2.1 a). ii) Un poro cilíndrico infinitamente largo, cargado, de diámetro interior $2 R$, que también denotaremos por $\tau(=2 R)$ (ver Fig. $2.1 \mathrm{~b}$ ). Los poros tienen densidad de carga superficial uniforme $\sigma_{\text {in }}$ y $\sigma_{\text {out }}$ en las superficies interior y exterior, respectivamente. Por simplicidad hemos considerado $\sigma \equiv \sigma_{\text {in }}=\sigma_{\text {out }}$. Es importante mencionar que las posiciones de los iones están situadas en los centros de los iones, entonces la posición más cercana de un ión a la pared de cualquier poro es a la distancia de un radio iónico del centro del ión a la pared, considerando que la superficie del ión está en contacto con la pared. Para el caso de geometría cilíndrica, en el interior del poro la distancia de máximo acercamiento del ión-pared es $t=R-a / 2$ a partir del centro del poro cilíndrico. La distancia correspondiente para el poro de rendija plana es escrita como $t=h-a / 2$, a partir del centro de las dos placas en el eje de simetría. En ambas geometrías el ancho de la pared o de las placas es $d$. Cada uno de los los poros se estudian por separado, estos se encuentran inmersos en un electrolito con un modelo primitivo restringido (RPM), es decir en un fluido que está constituido de dos componentes (iones) que consisten en esferas duras cargadas de igual tamaño con diámetro $a$, con cargas opuestas concentradas cada una de ellas en un punto (en el centro de las esferas) $z_{+}$e y $z_{-}$e, siendo a la vez $z_{+}$y $z_{-}$las valencias para cationes y aniones, respectivamente.

De esta manera tenemos que la energía potencial de interacción entre dos partículas de especie $i$ y $m$, separadas por una distancia $r_{13}$, es 


$$
u_{i m}\left(r_{13}\right)= \begin{cases}\frac{z_{i} z_{m} e^{2}}{\epsilon r_{13}} & \text { for } r_{13} \geq a \\ \infty & \text { for } r_{13}<a\end{cases}
$$

\subsubsection{Teoría general HNC-MSA para una solución iónica en un poro cargado}

La teoría de un fluido confinado de $\mathrm{N}$ componentes puede derivarse a partir de $(\mathrm{N}+1)$ componentes con las ecuaciones integrales de Ornstein-Zernike aplicando el método directo $[23,40]$, i.e.,

$$
h_{i j}\left(\mathbf{r}_{21}\right)=c_{i j}\left(\mathbf{r}_{21}\right)+\sum_{m=1}^{N+1} \rho_{m} \int h_{i m}\left(\mathbf{r}_{23}\right) c_{m j}\left(\mathbf{r}_{13}\right) d v_{3},
$$

donde $c_{i j}\left(\mathbf{r}_{21}\right)$ y $h_{i j}\left(\mathbf{r}_{21}\right)=g_{i j}\left(\mathbf{r}_{21}\right)-1$ son respectivamente, las funciones de correlación directa y correlación total entre dos partículas de especies $i$ y $j$ con posiciones relativas $\mathbf{r}_{21}=\mathbf{r}_{2}-\mathbf{r}_{1} ; g_{i j}\left(\mathbf{r}_{21}\right)$ es la función de correlación por pares, $\rho_{m}$ es la concentración en el bulto de las especies $m$ e $i, j=1, \ldots, N+1$. Considerando al poro como una especie más en el fluido, definimos la especie $N+1=\alpha$, y por una consideración adicional de que esta especie se encuentra diluida en forma infinita $\left(\rho_{a} \rightarrow 0\right)$ entonces obtenemos

$$
h_{\alpha j}\left(\mathbf{r}_{21}\right)=c_{\alpha j}\left(\mathbf{r}_{21}\right)+\sum_{m=1}^{N} \rho_{m} \int h_{\alpha m}\left(\mathbf{r}_{23}\right) c_{m j}\left(\mathbf{r}_{13}\right) d v_{3}
$$

Usaremos la cerradura de cadena hipertejida (HNC) en la función de correlación directa entre el poro y el fluido, $c_{\alpha j}\left(\mathbf{r}_{21}\right)$, es decir,

$$
c_{\alpha j}\left(\mathbf{r}_{21}\right)=h_{\alpha j}\left(\mathbf{r}_{21}\right)-\beta u_{\alpha j}\left(\mathbf{r}_{21}\right)-\ln g_{\alpha j}\left(\mathbf{r}_{21}\right),
$$

donde $u_{\alpha i}\left(\mathbf{r}_{21}\right)$ es el potencial de interacción directa entre el poro y una partícula de especie $j, \beta=1 / k_{B} T, k_{B}$ es la constante de Boltzmann y $T$ la temperatura absoluta. La función de correlación directa entre dos partículas del fluido, $c_{m j}\left(\mathbf{r}_{13}\right)$, es tomado en cuenta a través de la aproximación esférica media (MSA) para un electrolito RPM dada como

$$
c_{m j}^{M S A}\left(r_{13}\right)= \begin{cases}c_{s}\left(r_{13}\right)+z_{i} z_{m} c_{d}^{s r}\left(r_{13}\right)-\frac{z_{m} z_{j} \beta e^{2}}{\epsilon r_{13}}, & \text { for } r_{13}<a \\ -\frac{z_{m} z_{j} \beta e^{2}}{\epsilon r_{13}} & \text { for }\left|\mathrm{r}_{13}\right| \geq a\end{cases}
$$

siendo $c_{s}\left(r_{13}\right)$ y $c_{d}^{\text {rr }}\left(r_{13}\right)$ funciones de corto alcance dadas en el apéndice. Así pues, sustituyendo las ecs. (2.4) y (2.5) en la Eq. (2.3) nos queda

$$
\begin{aligned}
g_{\alpha j}\left(\mathbf{r}_{21}\right) & =\exp \left\{-\beta u_{\alpha j}\left(\mathbf{r}_{21}\right)+\sum_{m=1}^{N} \rho_{m} \int h_{\alpha m}\left(\mathbf{r}_{23}\right) c_{m j}^{M S A}\left(\mathbf{r}_{13}\right) d v_{3}\right\} \\
& =\exp \left\{-\beta u_{\alpha j}\left(\mathbf{r}_{21}\right)-\frac{z_{j} \beta e^{2}}{\epsilon} \int \rho_{\alpha d}\left(\mathbf{r}_{23}\right) \frac{d v_{3}}{r_{13}}\right. \\
& \left.+\frac{z_{j} \beta e^{2}}{\epsilon} \int \rho_{\alpha d}\left(\mathbf{r}_{23}\right) c_{d}^{s r}\left(r_{13}\right) d v_{3}+\int \rho_{\alpha s}\left(\mathbf{r}_{23}\right) c_{s}\left(r_{13}\right) d v_{3}\right\}
\end{aligned}
$$

donde nosotros hemos definido 


$$
\begin{gathered}
\rho_{\alpha d d}\left(\mathbf{r}_{13}\right)=\sum_{m=1}^{N} z_{m} \rho_{m} h_{\alpha m n}\left(\mathbf{r}_{13}\right) \\
\rho_{\alpha s}\left(\mathbf{r}_{13}\right)=\sum_{m=1}^{N} \rho_{m} h_{o m}\left(\mathbf{r}_{13}\right)
\end{gathered}
$$

Las ecs. (2.6) han sido derivadas para el caso general y se refieren a las ecuaciones integrales HNC/MSA, para partículas de especie $j$ cerca de la partícula de especie $\alpha$. Más adelante aplicaremos la ec. (2.6) a un electrolito RPM confinado en los poros de rendija plana y cilíndrica. También analizaremos los casos límites de iones puntuales y de poros de tamaño infinito.

\subsubsection{Teoría HNC/MSA para una solución iónica en un poro car- gado de rendija plana}

Para un poro de rendija plana consideramos la especie $\alpha$ hecha de dos placas paralelas infinitas cargadas (ver Fig. 2.1 a). De aquí que, en geometría plana, $u_{\alpha j}\left(\mathbf{r}_{21}\right)$ y $g_{\alpha j}\left(\mathbf{r}_{21}\right)$, dependen solamente de la coordenada perpendicular a la pared, $x$, es decir, $u_{a j}\left(\mathbf{r}_{21}\right)=u_{a j}(x)$ y $g_{\alpha j}\left(\mathbf{r}_{21}\right)=g_{\alpha j}(x)$. Además, el potencial de interacción puede ser separado de la siguiente manera

$$
u_{a j}(x)=u_{\alpha j}^{*}(x)+u_{\alpha j}^{e l}(x)
$$

donde $u_{a j}^{*}(x)$ y $u_{a j}^{e l}(x)$ son los términos de interacción de esfera dura y electrostático, respectivamente, que puede escribirse como

$$
u_{a j}^{*}(x)= \begin{cases}0 & \text { for }|x| \in\{[0, h-a / 2] \cup[h+d+a / 2, \infty)\} \\ \infty & \text { for }|x| \in\{(h-a / 2, h+d+a / 2)\}\end{cases}
$$

y

$$
-\beta u_{\alpha j}^{e l}(x)=\frac{2 \pi \beta e z_{j}}{\varepsilon} \sigma[2(x+h+d / 2)+|x-h|+|x-h-d|]
$$

para $0 \leq x<h \circ h+d<x$. Eq. (2.10) implica que $g_{\alpha j}(x)=0$ y $h_{a j}(x)=-1$ para $|x| \in\{(h-a / 2, h+d+a / 2)\}$. considerando la Ec. (2.6) para el poro de rendija plana; en este caso es conveniente usar coordenadas cilíndricas, centrado en el eje de simetría del poro, con la coordenada radial $r$, paralela a las placas y la coordenada axial, $y$, perpendicular a las placas, tal que, $r_{13}^{2}=x^{2}+y^{2}-2 x y$ y $d v_{3}=r d r d y d \phi$, siendo $\phi$ la coordenada angular y $x$ la coordenada axial para la partícula 1 (ver Fig.2.1 a). Entonces realizando un poco de álgebra obtenemos, (ver ref. $[40,79])$

$$
\begin{aligned}
g_{a j}(x) & =\exp \left\{-\beta z_{i} \epsilon \psi(x)+2 \pi \int_{\mathcal{D}} K(x, y) \rho_{\alpha s}(y) d y\right. \\
& \left.+2 \pi z_{j} \int_{\mathcal{D}} L(x, y) \rho_{\alpha d}(y) d y+A(x)\right\}
\end{aligned}
$$

donde el dominio de integración es $\mathcal{D}:=\{y \in[0, h-a / 2] \cup[h+d+a / 2, \infty)\}$ hemos definido $A(x) \equiv-2 \pi \rho_{T} \int_{h-a / 2}^{h+d d+\alpha} K(x, y)(y) d y, \rho_{T}=\sum_{m=1}^{2} \rho_{m}, \mathbf{y}$

$$
K(x, y) \equiv \int_{|x-y|}^{\infty} s c_{s}(s) d s
$$




$$
L(x, y) \equiv \int_{|x-y|}^{\infty} s c_{d}^{s r}(s) d s
$$

Las expresiones para $K(x, y)$ y $L(x, y)$ están dadas en la referencia |79]. El potencial electrostático medio, $\psi(x)$, está dado por

$$
\begin{aligned}
\psi(x) & =-\frac{2 \pi}{\epsilon} \sigma[2(x+h+d / 2)+|x-h|+|x-h-d|] \\
& -\frac{2 \pi e}{\epsilon} \int_{\mathcal{D}}(x+y+|x-y|) \rho_{\alpha d}(y) d y
\end{aligned}
$$

En términos del potencial al plano interior de máximo acercamiento $\zeta \equiv \psi(x=h-a / 2)$, la ec. (2.14) puede ser reescrita como

$$
\psi(x)=\zeta-\frac{2 \pi e}{\epsilon} \int_{0}^{h-\alpha / 2}(x+y-2 h+a+|x-y|) \rho_{\alpha d}(y) d y
$$

para $0 \leq x<h-a / 2$. En términos de la densidad de carga superficial, $\sigma$, el potencial $\zeta$ está dado por

$$
\zeta=-\frac{4 \pi}{\epsilon} \sigma d+\frac{2 \pi e}{\epsilon} a \int_{0}^{h-\alpha / 2} \rho_{\alpha d}(y) d y+\frac{4 \pi e}{\epsilon} \int_{h+d+a / 2}^{\infty}(h-y) \rho_{\alpha d}(y) d y
$$

para $0 \leq x<h-a / 2$.

\subsubsection{Teoría HNC-MSA para una solución iónica en un poro car- gado de forma cilíndrica}

Para este caso la especie $\alpha$ es una partícula coloidal cilíndrica infinitamente larga a dilución infinita (ver Fig.2.1 b). Ya con esto, resulta natural usar coordenadas cilíndricas para integrar la ec. (2.6), tal que, $r_{13}^{2}=x^{2}+y^{2}+z^{2}-2 x y \cos \phi$ y $d v_{3}=y d \phi d y d z$, siendo $\phi$, $z$, y $y$ las coordenadas angular, axial y radial para las particula 3 , respectivamente; $x$ es la coordenada radial para la partícula 1. Para las variables $\phi$ y $z$, la Ec. (2.6) puede ser integrada analíticamente (ver ref.[81]). Las expresiones para $u_{\alpha j}\left(\mathbf{r}_{21}\right)$ y $g_{\alpha j}\left(\mathbf{r}_{21}\right)$ dependen solamente de la coordenada radial, es decir, $u_{\alpha j}\left(\mathbf{r}_{21}\right)=u_{\alpha j}(x)$ y $g_{\alpha j}\left(\mathbf{r}_{21}\right)=g_{\alpha j}(x)$. Adicionalmente, el potencial de interacción puede ser separado como en la ec. (2.9), donde los términos correspondientes de interacción de esfera dura y electrostática son expresados como

$$
u_{\alpha j}^{*}(x)= \begin{cases}0 & \text { for } x \in\{[0, R-a / 2] \cup[R+d+a / 2, \infty)\} \\ \infty & \text { for } x \in\{(R-a / 2, R+d+a / 2)\}\end{cases}
$$

y

$$
u_{a i}^{e l}(x)=\left\{\begin{array}{l}
\frac{-4 \pi e z_{i}}{\epsilon} \sigma\left[R \ln (R)+(R+a+d) \ln (R+d)(2 R+d) \ln \left(x_{\infty}\right)\right] \\
\text { for } 0 \leq x \leq R-a / 2 \\
\frac{-4 \pi e z_{i}}{\epsilon} \sigma(2 R+d)\left[\ln x-\ln \left(x_{\infty}\right)\right] \\
\text { for } x \geq R+d+a / 2
\end{array}\right.
$$


siendo $x_{\infty}$ el punto de referencia de potencial electrostático cero, y el dominio de integración está definido como $\mathcal{D}:=\{y \in[0, R-a / 2] \cup[R+d+a / 2, \infty)\}$. Eq. (2.17) dando $g_{a j}(x)=0$ para $|x| \in\{(R-a / 2, R+d+a / 2)\}$, el cual también es tomado en cuenta en los límites de integración de la ec. (2.6). La ecuación HNC/MSA para el poro cilíndrico, después de realizar la correspondiente álgebra, es escrita como

$$
\begin{aligned}
g_{\alpha j}(x) & =\exp \left\{-e \beta z_{j} \psi(x)+\int_{\mathcal{D}} K_{c}(x, y) \rho_{\alpha s}(y) d y\right. \\
& \left.+z_{j} \int_{\mathcal{D}} L_{c}(x, y) \rho_{\alpha d}(y) d y+A_{c}^{\prime}(x)\right\}
\end{aligned}
$$

De manera similar como la ec. (2.13) nosotros hemos definido,

$$
\begin{aligned}
K_{c}(x, y) & \equiv \int_{-\infty}^{\infty} \int_{0}^{2 \pi} c_{s}(s) y d \phi_{3} d z_{3} \\
L_{c}(x, y) & \equiv \int_{-\infty}^{\infty} \int_{0}^{2 \pi} c_{s}^{s r}(s) y d \phi_{3} d z_{3}
\end{aligned}
$$

(ver el Apéndice), siendo el potencial electrostático medio, $\psi(x)$, dado como

$$
\begin{aligned}
\psi(x) & =-\frac{4 \pi}{\epsilon} \sigma[R \ln R+(R+d) \ln (R+d)] \\
& -\frac{4 \pi e}{\epsilon} \int_{\mathcal{D}} \ln \left(\frac{x^{2}+y^{2}+\left|x^{2}-y^{2}\right|}{2}\right) \rho_{o d}(y) y d y
\end{aligned}
$$

para $0 \leq x \leq R-a / 2, y$

$$
\begin{aligned}
\psi(x) & =-\frac{4 \pi}{\epsilon} \sigma(2 R+d) \ln x+ \\
& +\frac{2 \pi e}{\epsilon} \int_{\mathcal{D}} \ln \left(\frac{x^{2}+y^{2}+\left|x^{2}-y^{2}\right|}{2}\right) \rho_{\alpha d}(y) y d y
\end{aligned}
$$

para $x \geq R+d+a / 2$. En términos del potencial en el interior a la distancia al plano de máximo acercamiento, $\zeta \equiv \psi(x=R-a / 2)$, el potencial electrostático medio, para $x<R-a / 2$, puede ser escrito como

$$
\psi(x)=\zeta-2 \pi \int_{0}^{R-u / 2} \ln \left(\frac{x^{2}+y^{2}+\left|x^{2}-y^{2}\right|}{2(R-a / 2)^{2}}\right) y \rho_{\alpha d}(y) d y
$$

En términos de la densidad de carga superficial, el potencial- $\zeta$, está dado por

$$
\begin{aligned}
\zeta & =-\frac{4 \pi e}{\epsilon} \sigma(R+d) \ln \left(\frac{R+d}{R}\right)-\frac{4 \pi e}{\epsilon} \int_{R+d+a / 2}^{\infty} \ln \left(\frac{y}{R}\right) \rho_{\text {add }}(y) y d y \\
& +\frac{4 \pi \epsilon}{\epsilon} \ln \left(\frac{R}{R-a / 2}\right) \int_{0}^{R-a / 2} \rho_{a d}(y) y d y
\end{aligned}
$$


De esta forma, es interesante notar que $\psi(x)$, cuando es expresado en términos de $\sigma$, las regiones interna y externa del poro están acopladas a través de los perfiles de concentración reducida de iones, $g_{a j}(x)$ |ver Eq. (2.14) para el poro de rendija plana, y las Ecs. (2.21) y (2.22) para el poro cilíndrico]. Así de esta forma, si $\psi(x)$ es expresado en términos de $\sigma$, las ecuaciones HNC/MSA [Ecs. (2.12) y (2.19)] deben ser resueltas consistentemente por considerar las regiones interna y externa. Por otro lado, si el $\psi(x)$ es escrito en términos de $\zeta$, las ecuaciones HNC/MSA para las regiones interna y externa pueden ser resueltas independientemente. Sin embargo, debe ser señalado que ambas perspectivas, es decir términos de $\zeta$ o $\sigma$, son equivalentes.

\subsubsection{Casos Límites de tamaño de poros infinitos e iones puntuales}

Para el caso límite de poros infinitos tenemos que, si tomamos el límite de $R \rightarrow \infty$, para la geometría cilíndrica o $h \rightarrow \infty$, para el poro de rendija plana, se puede mostrar que las ecuaciones HNC/MSA, es decir Ecs. (2.12) y (2.19), van a ser

$$
\begin{aligned}
g_{a j}(x) & =\exp \left\{-\beta z_{i} e \psi(x)+2 \pi \int_{\mathcal{D}} K(x, y) \rho_{\alpha s}(y) d y\right. \\
& \left.+2 \pi z_{j} \int_{\mathcal{D}} L(x, y) \rho_{\alpha d}(y) d y-2 \pi A(x)\right\}
\end{aligned}
$$

donde el dominio de integración es $\mathcal{D}:=\{y \in[a / 2, \infty)\}, x$ es la posición de la partícula medida desde la superficie de la pared; $A(x), K(x, y)$, y $L(x, y)$ tienen la misma forma como en la Ec. (2.13) del nanoporo de rendija plana. Por esto es importante señalar que la Ec. (2.25 es la ecuación HNC/MSA para un electrolito RPM cerca de una pared plana de espesor infinito y se satisface en ambos lados de la pared. En términos de la densidad de carga superficial $\sigma$, el potencial electrostático medio se escribe como

$$
\psi(x)=\frac{4 \pi}{\epsilon} \sigma x-\frac{2 \pi e}{\epsilon} \int_{a / 2}^{\infty}[x+y+|x-y|] \rho_{\alpha d}(y) d y
$$

o equivalentemente,

$$
\psi(x)=\zeta-\frac{2 \pi e}{\epsilon} \int_{a / 2}^{\infty}[a-x-y+|x-y|] \rho_{\alpha d}(y) d y
$$

en términos del potencial $\zeta$.

La ecuación de Poisson-Boltzmann (PB) se obtiene despreciando las correlaciones de corto alcance, tomando el diámetro iónico igual a cero, es decir, $a=0$, que es equivalente a tomar $K(x, y), L(x, y)$ y $A(x)$ igual a cero en las Ecs. (2.12) y $(2.25) \circ K_{c}(x, y), L_{c}(x, y)$ y $A_{c}^{\prime}(x)$, igual a cero en Ec. (2.19). Por lo tanto, la forma compacta de la ecuación de PB puede ser escrita como

$$
g_{\alpha j}^{P B}(x)=e^{-\beta e z_{j} \psi(x)}
$$

donde $\psi(x)$ es el potencial electrostático medio, dado por las Ecs. (2.14) y (2.15) para el poro de rendija plana. por las Ecs. $(2.21),(2.22),(2.23)$ para el poro cilíndrico y por las ecs. (2.26) y (2.27) para una pared simple. 


\subsubsection{Ecuaciones para las propiedades electrocinéticas para un mi- croporo de forma de rendija de 2 placas}

Las expresiones para los coeficientes electrocinéticos dentro de un poro de rendija plana, que se encuentra dentro de una solución cargada, se obtienen a partir de ecuaciones que se relacionan con las propiedades electrocinéticas del fluido.

La aplicación de un gradiente de presión, $P_{z}$, y/o un gradiente de potencial eléctrico, $E_{z}$, través de un capilar de forma de rendija con dos placas planas cargadas, en la dirección $z$, (paralela a las placas) y en cuyo volumen se encuentra ocupado por un electrolito; se genera un flujo de volumen neto de solución $V$ y la aparición de una corriente eléctrica neta $I$ a través de la sección transversal del poro. Hemos considerado que los efectos anteriores tienen dependencia lineal con $E_{z}$ y $P_{z}$ y están acoplados a través de un conjunto de variables, conocidos como coeficientes de transporte de Onsager, $L_{m n}$, que son expresados en dos ecuaciones lineales de la siguiente manera

$$
V=L_{11} P_{z}+L_{12} E_{z}
$$

y

$$
I=L_{21} P_{z}+L_{22} E_{z}
$$

La ecuación de Navier-Stokes para el flujo en estado estacionario de un fluido viscoso a través de un poro cilindrico, sometido a los esfuerzos $E_{z}$ y $P_{z}$, es

$$
\eta\left(\frac{d u^{2}(x)}{d x^{2}}\right)+\rho_{d}(x) E_{z}+P_{z}=0
$$

donde $u(x)$ es la velocidad del flujo en la dirección $z$, como una función de la coordenada perpendicular a las placas $x$; $\eta$ es el coeficiente de viscosidad en el bulto de la solución electrolítica, y $\rho_{d}(x)$, es densidad de carga local definida por

$$
\rho_{d}(x)=\sum_{m=1}^{N} z_{m} e \rho_{m}(x) .
$$

Integrando la Ec. (2.31), donde la velocidad debe satisfacer las siguientes condiciones de frontera

$$
\begin{aligned}
& u(x)=0, \quad \psi(x)=\zeta \text { en } x=t \\
& \frac{d u(x)}{d x}=0, \quad \frac{d \psi(x)}{d x}=0 \text { en } x=0
\end{aligned}
$$

con $\zeta=\psi(h-a / 2), y$ donde $t \equiv h-a / 2$. Usando la ecuación de Poisson la densidad de carga local es

$$
\rho_{d}(x)=-\frac{\varepsilon}{4 \pi}\left(\frac{d \psi^{2}(x)}{d x^{2}}\right)
$$

y definiendo

$$
u(x)=u_{p}(x)+u_{E}(x)
$$


tenemos

$$
u(x)=-\frac{1}{2 \eta}\left(x^{2}-h^{2}\right) P_{z}-\mu\left(1-\frac{\psi(x)}{\zeta}\right) E_{z}
$$

donde

$$
\begin{aligned}
& u_{p}(x)=-\frac{1}{2 \eta}\left(x^{2}-h^{2}\right) P_{z} \\
& u_{E}(x)=-\mu\left(1-\frac{\psi(x)}{\zeta}\right) E_{z}
\end{aligned}
$$

$y, \mu \equiv \frac{\varepsilon \zeta}{4 \pi \eta}$ es la movilidad eletroforética. Los dos términos en la ecuación ( 2.36) son las componentes de la velocidad de Poiseuille y electro-osmótica respectivamente.

El flujo de volumen total por unidad de superficie $V$, también llamado flujo de volumen electro-osmótico se obtiene integrando $u(x)$ sobre la sección transversal total entre las dos placas, es decir,

$$
V=\int_{-1}^{t} u(x) d x
$$

Así, subtituyendo, Ec. (2.36) en la Ec. (2.39), y de acuerdo a la Ec. (2.29) se obtiene

$$
L_{11}=\frac{2 t^{3}}{3 \eta}
$$

$\mathbf{y}$

$$
L_{12}=L_{12}^{\infty}(1-G)
$$

donde

$$
G=\frac{1}{\zeta t} \int_{0}^{t} \psi(x) d x
$$

y

$$
L_{12}^{\infty}=-2 t \mu \text {. }
$$

siendo $L_{12}^{\infty}$ la razón $V / E_{z}$ para $P_{z}=0$ en el límite de Smoluchowski, es decir $h \rightarrow \infty$.

La velocidad de los iones de especie $j$, bajo el potencial eléctrico y el gradiente de presión, tiene dos componentes una convectiva y otra de transporte respectivamente, escritas como

$$
u_{j}(x)=u(x)+u_{j}^{T}(x)
$$

Donde el primer término del lado derecho de la ecuación es la velocidad de flujo de toda la solución, dada por la ec. (2.36), y el segundo término es la velocidad de transporte debido a la movilidad iónica, $\mu_{j}=\frac{t\left|z_{j}\right|}{f_{j}}$, escrito de la forma

$$
u_{j}^{T}(x)=\frac{z_{j}}{\left|z_{j}\right|} \mu_{j} E_{2}
$$

siendo $f_{j}$ el coeficiente de fricción, de las especies cargadas $j$ sometidas al campo eléctrico $E_{z}$. 
El flujo de corriente total es

$$
I=2 \sum_{j} z_{j} e \int_{0}^{t} u_{j}(x) \rho_{j}(x) d x
$$

que puede expresarse como

$$
I=I_{c}+I_{T}
$$

donde se define

$$
\begin{gathered}
I_{c}=I_{c}^{p}+I_{c}^{E} \\
I_{c}=2 \int_{0}^{t} u(x) \rho_{d}(x) d x \\
I_{c}=2 \int_{0}^{t} u_{p}(x) \rho_{d}(x) d x+2 \int_{0}^{t} u_{E}(x) \rho_{d}(x) d x
\end{gathered}
$$

donde

$$
\begin{aligned}
& I_{c}^{p}=2 \int_{0}^{t} u_{p}(x) \rho_{d}(x) d x \\
& I_{c}^{E}=2 \int_{0}^{t} u_{E}(x) \rho_{d}(x) d x
\end{aligned}
$$

y el último término es

$$
I_{T}=2 E_{z} \int_{0}^{t} e \sum_{j} z_{j} \rho_{j}(x) u_{j}^{T}(x) d x
$$

usando las ecs. Ec. (2.37), Ec. (2.34, integrando por partes y las condiciones de frontera, tenemos la componente de la corriente de convección para $P_{z}$ es

$$
I_{c}^{p}=L_{12} P_{z}
$$

Sustituyendo la velocidad electro-ósmotica $u_{E}(x)$ de la ec. (2.38) en la ec. $(2.52$, tenemos la componente de la corriente de convección para $E_{z}$ es

$$
I_{c}^{E}=L_{12}^{\infty} E_{z} J_{c}
$$

donde

$$
\begin{gathered}
J_{c}=\frac{1}{h} \int_{0}^{t} \rho_{d}(x)\left(1-\frac{\psi(x)}{\zeta}\right) d x \\
I_{T}=2 h \sigma_{T} E_{z}\left(1+K_{T}\right)
\end{gathered}
$$

donde

$$
K_{T}=\frac{1}{t} \sum_{j} t_{j} \int_{0}^{t} h_{j}(x) d x
$$

definiendo

$$
K^{*}=K_{T}+K_{c}
$$


$K$ es el coeficiente de conductancia capilar, que tiene una contribución convectiva y otra de transporte iónico, donde $K_{c}$ está dada por

$$
K_{c}=\frac{L_{12}^{\infty}}{L_{22}^{\infty}} J_{c}
$$

en términos de los coeficientes de Onsager, la corriente total está dada por la Ec. (2.30),con

$$
L_{22}=L_{22}^{\infty}(1+K) \text {, }
$$

donde $L_{22}^{\infty}=2 t \sigma_{T}$

$$
K=\frac{1}{t}\left\{\sum_{j} t_{j} \int_{0}^{t} h_{j}(x) d x-\frac{\mu}{\sigma_{T}} \int_{0}^{t} \rho_{d}(x)\left(1-\frac{\psi(x)}{\zeta}\right) d x\right\},
$$

donde $t_{j}=\frac{\sigma_{j}}{\sigma_{T}}$ es el coeficiente de transporte iónico de la especie $j, \sigma_{T}=\sum \sigma_{j}$ es la conductividad de la solución en el bulto, y $\sigma_{j}$ es la conductividad iónica de la especie $j$ definida como $\sigma_{j}=\rho_{j}\left|z_{j}\right| \mu_{j}$ e. También se cumple $L_{12}=L_{21}$.

La diferencia de potencial $E_{z}$ producida cuando un gradiente de presión $P_{z}$ se aplica a través de un capilar, bajo la condición de corriente eléctrica $I$, cero, se refiere a un potencial de corriente $E_{s t r}=\left(E_{z}\right)_{l=0}$. El potencial de corriente, $E_{s t r}$ está dado usando las ecuaciones Ec. $(2.30),(2.41), y(2.61)$ como

$$
E_{s t r}=-\frac{L_{12}}{L_{22}} P_{z}=\frac{\varepsilon \zeta_{a}}{4 \pi \eta \sigma_{T}} P_{z}
$$

donde $\zeta_{a}=\zeta F$ es el potencial $\zeta$ aparente y

$$
F=\frac{1-G}{1+K}
$$

es el potencial $\zeta$ aparente reducido.

Si la condición de corriente cero se aplica en la Ec. (2.29) y usando la Ec. (2.63). La razón del flujo de volumen con la presión, en términos de los coeficientes de Onsager, está dada por

$$
\left(\frac{V}{P_{z}}\right)_{I=0}=L_{11}\left[1+\frac{L_{12}^{2}}{L_{11} L_{22}}\right]
$$

Entonces, el flujo de volumen efectivo de Poiseuille es

$$
V=\frac{2 t^{3}}{3 \eta_{a}} P_{z}
$$

donde se define un coeficiente de viscosidad aparente, $\eta_{a}$, para mantener la forma de la fórmula de Poiseuille para el flujo de volumen en la Ec. (2.40). De este modo, por combinación de las Ecs. (2.40), (2.41), (2.43), (2.65), y (2.66) resulta 


$$
\frac{\eta_{a}}{\eta}=\left[1-3 \beta^{*}\left(\frac{\zeta^{*}}{\kappa t}\right)^{2} \frac{(1-G)^{2}}{1+K}\right]^{-1}
$$

donde $\zeta^{*}=\zeta e \beta$ y definiendo

$$
\beta^{*}=\left(\frac{\varepsilon}{4 \pi}\right)^{2}\left(\frac{k_{B} T}{e}\right)^{2} \frac{\kappa^{2}}{\eta \sigma_{T}}
$$

y $\kappa=\sqrt{\frac{4 \pi e^{2}}{\varepsilon k_{B} T} \sum_{i} \rho_{i} z_{i}^{2}}$ es la inversa de la longitud de Debye.

\subsubsection{Ecuaciones para las propiedades electrocinéticas para un mi- croporo cilíndrico}

Las expresiones para los coeficientes electrocinéticos dentro de un poro de rendija plana y sus resultados obtenidos por las aproximaciones de HNC/MSA y PB, han sido discutidas anteriormente, ver ref. $([9,10,83])$. En esta parte nosotros obtenemos las ecuaciones para las propiedades electrocinéticas dentro de un poro cilíndrico con un procedimiento similar a la referencia anterior. Consideremos el eje del poro cilíndrico (o simplemente el cilindro o el poro) como el eje axial de coordenadas, z. La aplicación de un gradiente de presión, $P_{z}=-\nabla_{z} P(z), y / 0$ un gradiente de potencial eléctrico, $E_{z}=-\nabla_{z} E(z)$, a través de un capilar cargado, en la dirección axial $z$, genera un flujo de volumen neto $V$ y una corriente eléctrica neta $I$ a través de la sección transversal del poro. Hemos considerado que $E_{z}$ y $P_{z}$ están acoplados a través de un conjunto de coeficientes de transporte de Onsager, $L_{m n}$. Estas ecuaciones son lineales y están expresadas como

$$
V=L_{11} P_{z}+L_{12} E_{z}
$$

y

$$
J=L_{21} P_{z}+L_{22} E_{z}
$$

La ecuación de Navier-Stokes para el flujo en estado estacionario de un fluido viscoso a través de un poro cilíndrico, sometido a los esfuerzos $E_{z}$ y $P_{z}$, es

$$
\eta \frac{1}{x} \frac{d}{d x}\left(x \frac{d u(x)}{d x}\right)+\rho_{d}(x) E_{z}+P_{z}=0
$$

donde $u(x)$ es la velocidad del flujo en la dirección $z$, como una función de la coordenada radial $x: \eta$ es el coeficiente de viscosidad en el bulto de la solución electrolítica, y $\rho_{d}(x)$, es densidad de carga local definida por

$$
\rho_{d}(x)=\sum_{m=1}^{N} z_{m} e \rho_{m}(x)
$$

Integrando la Ec. (2.71), donde la velocidad debe satisfacer las siguientes condiciones de frontera 


$$
\begin{aligned}
& u(x)=0, \quad \psi(x)=\zeta \quad \text { en } x=t \\
& \frac{d u(x)}{d x}=0, \quad \frac{d \psi(x)}{d x}=0 \text { en } x=0
\end{aligned}
$$

con $\zeta=\psi(R-a / 2), y$ donde $t \equiv R-a / 2$. Usando la ecuacion de Poisson la densidad de carga local es

$$
\rho_{d}(x)=-\frac{\varepsilon}{4 \pi} \frac{1}{x} \frac{d}{d x}\left(x \frac{d y}{d x}\right)
$$

se obtiene

$$
u_{z}(x)=-\frac{1}{4 \eta}\left(x^{2}-R^{2}\right) P_{z}-\mu\left(1-\frac{\varphi(x)}{\zeta}\right) E_{z}
$$

donde $\mu \equiv \frac{\varepsilon \zeta}{4 \pi \eta}$ es la movilidad eletroforética. Los dos términos en la ecuación ( 2.75$)$ son las componentes de la velccidad de Poiseuille y electro-osmótica respectivamente.

El flujo de volumen total por unidad de superficie $V$, también llamado flujo de volumen electro-osmótico se obtiene integrando $u(x)$ sobre là sección transversal total del cilindro, es decir,

$$
V=2 \pi \int_{c}^{t} u(x) a d x
$$

Así, subtituyendo. Ec. (2.75) en la Ec. (2.70), y de acuerio a la De. (2.29) se obtiene

$$
\begin{gathered}
L_{1 !}=\frac{A i^{2}}{8 \eta} \\
L_{12}=L_{12}^{\infty}(1-G)
\end{gathered}
$$

donde

$$
G=\frac{2}{\zeta t^{2}} \int_{0}^{t} v(x) x d 2
$$

y

$$
L_{12}^{x}=-A \mu
$$

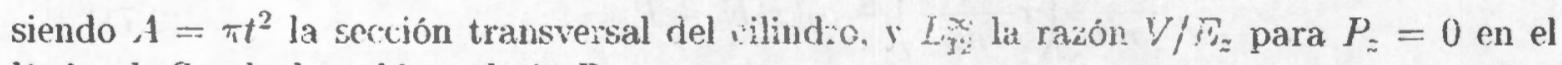
límite de Smoluchor:ski, es decir $R \rightarrow x$.

La velocidad de los iones de especie $j$. bajo el potencial clectrico y el gradiente de presión, tiene dos conıpelientes una convectiva y otua de transporte respecrivamente, escritas como

$$
u_{j}(x)=u(x)+u_{j}^{T}(x)
$$

Donde el primer término del lado derecho de la couación as la rolucidad de flujo de toda la solución, dada por la ec. (2.75), y el segundón témino es la velocidzud de transporte debido a la movilidad iónica, $\mu_{j}=\frac{e_{\mid}^{\mid} \tilde{z}_{j} \mid}{f_{3}}$. escrito de la forme. 


$$
u_{j}^{T}(x)=\frac{z_{j}}{\left|z_{j}\right|} \mu_{j} E_{z}
$$

siendo $f_{j}$ el coeficiente de fricción, de las especies cargadas $j$ sometidas al campo eléctrico $E_{z}$. Por tanto el flujo de corriente total es

$$
I=\sum_{j} 2 \pi z_{j} e \int_{0}^{t} u_{j}(x) \rho_{j}(x) x d x
$$

o también

$$
I=I_{c}+I_{T}
$$

donde

$$
I_{c}=2 \pi \int_{0}^{t} u(x) \rho_{d}(x) x d x
$$

y

$$
I_{T}=2 \pi E_{z} e \sum_{j} m_{j}\left|z_{j}\right| \int_{0}^{t} \rho_{j}(x) x d x
$$

Así de esta manera, sustituyendo la Ec. (2.75) en la Ec. (2.85), la corriente total Ec. (2.47), puede escribirse en términos de los coeficientes de Onsager, ver Ec. (2.30), siendo

$$
\begin{gathered}
L_{21}=-2 \pi \int_{0}^{t} \frac{1}{4 \eta}\left(x^{2}-t^{2}\right) \rho_{d}(x) x d x \\
L_{22}=L_{22}^{\infty}(1+K) \\
K=\frac{2}{t^{2}}\left\{\sum_{j} t_{j} \int_{0}^{t} h_{j}(x) x d x-\frac{\mu}{\sigma_{T}} \int_{0}^{t} \rho_{d}(x)\left(1-\frac{\psi(x)}{\zeta}\right) x d x\right\}
\end{gathered}
$$

y

$$
L_{22}^{\infty} \equiv A \sigma_{T}
$$

donde $K$ es el coeficiente de conductancia capilar, llamada a veces conductancia superficial, $t_{j}=\frac{\sigma_{j}}{\sigma_{T}}$ es el coeficiente de transporte iónico de la especie $j, \sigma_{T}=\sum \sigma_{j}$ es la conductividad de la solución en el bulto, y $\sigma_{j}$ es la conductividad iónica de la especie $j$ definida como $\sigma_{j}=\rho_{j}\left|z_{j}\right| \mu_{j}$ e. Se puede probar que $L_{12}=L_{21}$.

Una cantidad que puede ser medida experimentalmente es la razón del volumen con el flujo de corriente para gradiente de presión igual a cero, es decir,

$$
\begin{aligned}
\left(\frac{V}{I}\right)_{P_{2}=0} & =\frac{L_{12}^{\infty}}{L_{22}^{\infty}}\left[\frac{1-G}{1+K}\right] \\
& =-\frac{\epsilon \zeta_{a}}{4 \pi \eta \sigma_{T}}
\end{aligned}
$$

donde $\zeta_{u}=\zeta F$ es el potencial $\zeta$ aparente y

$$
F=\frac{1-G}{1+k}
$$

es el potencial $\zeta$ aparente reducido. 
El potencial de corriente. $E_{s t r}$. está defiuido como la diferenciá de potencial inducida por la aplicación de un gradiente de presión a lo largo del poro, $P_{2}$, bejo la condición de corriente cero. Por la Ec. (2.30), (2.41), y (2.88) se obtiene

$$
E_{s t r}=-\frac{I_{12}}{L_{22}} P_{z}=\frac{\varepsilon \zeta_{y s}}{4 \pi r_{i} \sigma_{Y}} P_{z}
$$

Si la condición de corriente cero se apliç en la Ec. (2.29) y usaráo la Ec. (2.93). La razón del flujo de volumen con la presión, en términos de los coeficientes de Onsager, está dada por

$$
\left(\frac{V}{P_{z}}\right)_{l=0}=L_{11}\left[1+\frac{L_{12} L_{2 i}}{L_{11} L_{22}}\right]
$$

Entonces, el flujo de volumen efectivo de Poiseuille $\in$

$$
V=\frac{4}{8 \eta_{a}} t^{2} P_{z}
$$

donde se define un coeficiente de viscosidad aparene. ra nara mantener al forma de la fórmula de Poiseuille para el flujo de volumen en la Ec. (2.7i). De este nodo, por combinación de las Ecs. (2.77), (2.41), (2.80), (2.94). y (2.95) result?

$$
\frac{\eta_{a}}{\eta}=\left[1-8 \beta^{*}\left(\frac{\zeta^{*}}{k t}\right)^{2} \frac{(1-\zeta)^{-1}}{1+K}\right]
$$

donde $\zeta^{*}=\zeta e / k T$ y definiendo

$$
\beta^{*}=\left(\frac{E}{4 \pi}\right)^{2}\left(\frac{k_{B}{ }^{2}}{e}\right)^{2} \frac{\kappa^{2}}{r_{i} \tau_{i}}
$$

y $\kappa=\sqrt{\frac{4 \pi e^{2}}{\varepsilon k_{B} T} \sum_{i} \rho_{i} z_{i}^{2}}$ es la inversa de la longitud de Debye.

A continuación presentaremos un resumen de los cofici:rtes de transporte para un poro de rendija plana (placas paralelas) $\{9,10,83\}$.

$$
\begin{gathered}
L_{11}=\frac{L}{3 i_{i}} t^{2} \\
L_{12}=-L_{1 i}\left(1-C_{i}\right)
\end{gathered}
$$

$y$

$$
L_{22}=-L \sigma_{g}(1+h)
$$

donde $L=2 t, y$

$$
G=\frac{1}{\pi} \int_{0}^{t} x(x) d x
$$




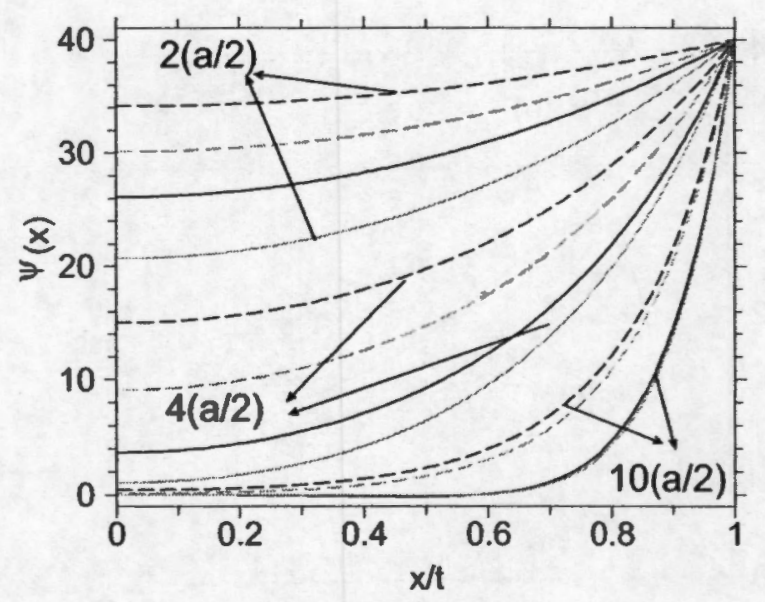

Figura 2.2: Perfil de potencial electrostático medio, $\psi(x)$, para un electrolito 1:1 en un modelo primitivo restringido (RPM) confinado en poros cilíndrico y de placas paralelas. la concentración del electrolito es $\rho_{s}=1 \mathrm{M}$, y el potencial a la distancia de máximo acercamiento de los iones a la pared es $\zeta \equiv \psi(x=t)=40 \mathrm{mV}$. Para los valores de diámetros de cilindro o separación de las placas que son considerados son $\tau=10 a, 4 a$ and $2 a$. La convención de las líneas es la siguiente: líneas continuas y punteadas corresponden a las teorías $\mathrm{HNC} / \mathrm{MSA}$ y $\mathrm{PB}$, respectivamente. Líneas Oscuras y claras corresponden al cilindro y a las placas paralelas, respectivamente. En todos los casos el diámetro de los iones es $a=4,25 \AA$, la temperatura es $T=298 \mathrm{~K}$ y la constante dieléctrica es $\epsilon=78,5$

$$
\begin{gathered}
K=\frac{1}{t}\left\{\sum_{j} t_{j} \int_{0}^{t} h_{j}(x) d x-\frac{\mu}{\sigma_{T}} \int_{0}^{t} \rho_{d}(x)\left(1-\frac{\psi(x)}{\zeta}\right) d x\right\} \\
\frac{\eta_{a}}{\eta}=\left[1-3 \beta^{*}\left(\frac{\zeta^{*}}{\kappa t}\right)^{2} \frac{(1-G)^{2}}{1+K}\right]^{-1}
\end{gathered}
$$

La notación está dada como en la Fig.2.1 b, y la expresión para $F$ es la misma que para la geometría cilíndrica dada por Eq. (2.92). En ambas geometrías, si tomamos el límite de $t \rightarrow$ $\propto$, puede demostrarse $L_{11}=\frac{2 t^{3}}{3 \eta}, L_{12}=-2 t \mu, y L_{22}=2 t \sigma_{T}$, es decir, nosotros recobramos los coeficientes de transporte de Onsager para un fluido cerca de una pared plana. Finalmente cabe mencionar que, desde el punto de vista de la teoría de $\mathrm{PB}$, ésto ha sido analizado para el régimen de potencial $\zeta$ bajo para el el poro cilíndrico $|41|$ y para el poro de rendija plana [78], dando origen a las expresiones analíticas simples de los coeficientes de transporte electrocinéticos, en términos de variables adimensionales $\zeta^{*}$ y $\kappa t$. 


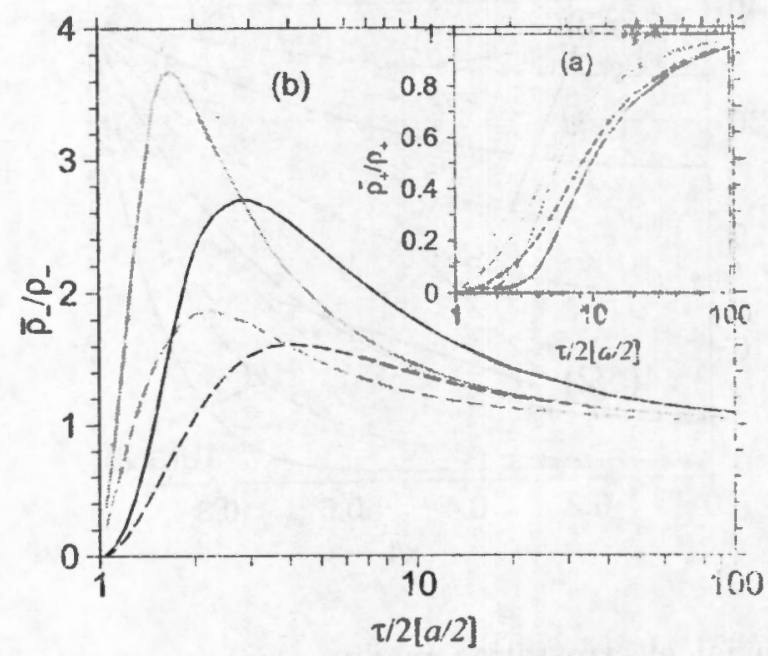

Figura 2.3: Promedio de contraiones reducido $\left(\bar{\rho}_{-} / \rho_{-}\right)$y concentación de coiones $\left(\bar{\rho}_{+} / \rho_{+}\right)$ dentro de los poros cilindrico y de rendija plana, como una furicion de su tamaño $\tau / 2$. La convención de los parámetros y las líneas es la misma que er la l. 5.2.2.

\subsubsection{Resultados y Discusión}

\section{POTENCIAL SUPERFICIAL CONSTANTE}

Se obtuvieron por medio de las teorías de HNC/MSA y $\mathrm{PB}$. loe perfiles de concentración iónica en equilibrio y el potencial electrostático medio para tun trolito con modelo primitivo restringido (RPM), confinado por poros cilíndrico y de rendija plana. De esta manera los coeficientes de transporte de Onsager y las propieclades electrovisáticas fueron calculadas. En todos los cálculos, a potencial superficial constante. el flectroito ps nonovalente (1:1), con la temperatura constante de de $T=298 \mathrm{~K}$, y el didrutro lo iones es $a=4.25 \AA$. Con el fin de apreciar mejor el efecto de las correlacioncs de car ic alc ance hemos elegido la concentración de bulto con el valor de $\rho_{s}=1 \mathrm{M}$. Sin enbargo importaites diferencias entre las teorias HNC/MSA y Poisson-Boltzmann, atribuidas a la concinaracion de correlaciones de corto alcance, por anterioridad, puede ser visto desde rere $a, \ldots \approx 0.1 \mathrm{M}$.

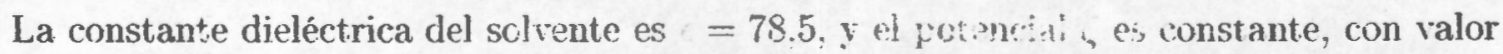
$\zeta=40 \mathrm{mV}$. Los cálculos fueron realizados para varios taratiri.. de poro. El plano de no deslizamiento del fluido, donde $u(x)=0$, por simplicidad fur tomado en $x=t \equiv R-a / 2$ y $x:=t \equiv h-a / 2$ para el cilindro y la rendija plana, respecivinert:

Las propiedades de transporte para lá rendịa plena lazr sibo estidiødas ampliamente, anteriormente (ver $[9,10,83]$ ). En este trabajo rosoiros compararremos las propiedades de transporte y de equilibrio para una rendija plana con lis inismas propiedades que para un cilindro. For tanto en Fig.2.2 se muestran los resultados nsadio a forids liNC MSA y PB de los perfiles de potencial electrostático medio, $v(x)$. La cist ancia. $x$. cs medida del centro del poro y ésta, es expresada en términos de lá distancia do muximo accrcamiento de los iones a la superficie de la pared del poro, $t$. Así, el plario de no desijzamiento está en $x / l=1$, 


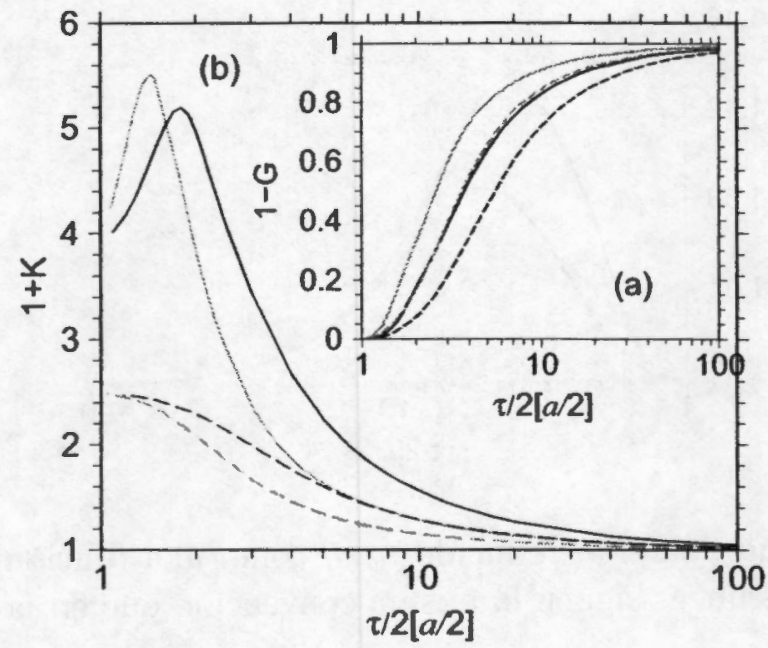

Figura 2.4: Flujo de volumen reducido, $1-G$ (fig. interior) y conductancia capilar reducida $1+K$ (figura principal), como una función del tamaño del poro. La convención para las líneas y los parámetros es la misma que en la figura Fig.2.2

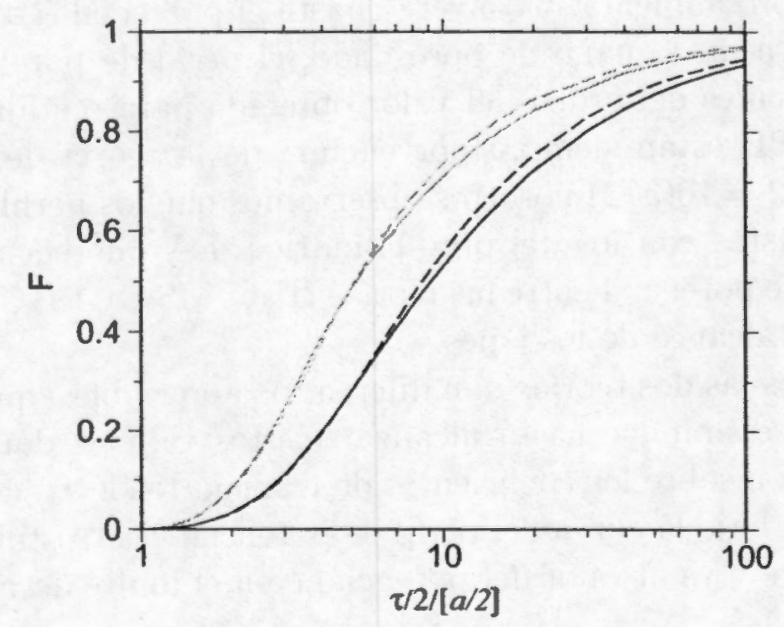

Figura 2.5: Coeficiente del potencial de corriente reducido $F$ como una función del tamaño del poro, $\tau / 2$. La convención para los parámetros y las líneas es la misma que en la figura Fig. 2.2 . 


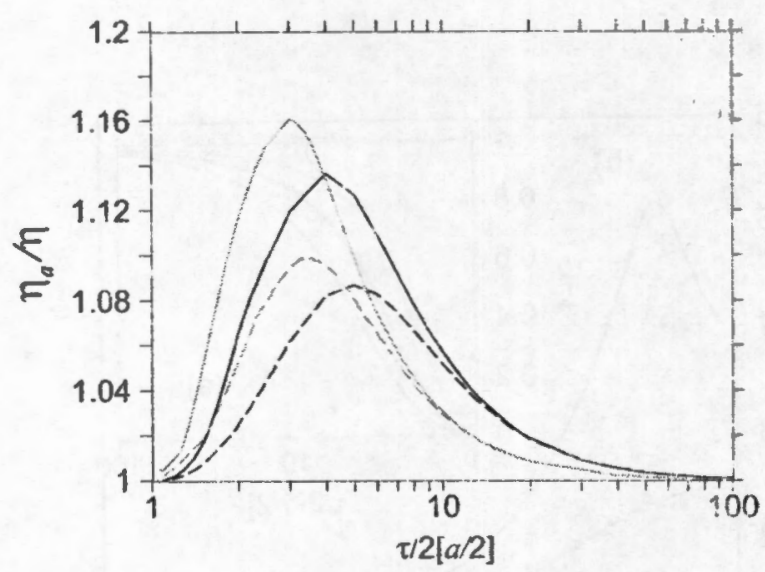

Figura 2.6: Electroviscosidad aparente reducida $\eta_{a} / \eta$ como ura función del tamaño de los poros. Los parámetros y las líneas siguen la misma convención que en la figura Fig.2.2.

en todos los casos. Aquí y más adelante isaremos la siguiente convención en las figuras: Resultados HNC/MSA son trazados con líneas continuas, mientras que las líneas punteadas para la teoria de PB. Las líneas obscuras son usadas para el poro cilíndrico mientras que las líneas claras se usan para representar los resultados del poro de rendija plana.

Los resultados son trazados para tres tamaños de poro son representados en la Fig.2.2, es decir, para $\tau / 2=2(a / 2), 4(a / 2)$ y $10(a / 2)$, como se indica en la figura. En todos los casos los perfiles de potencial electrostático medio se comportan monotónicamente crecientes cuando la disrancia al centro del poro aumenta, y coliverge hast,a el potencial $\zeta \equiv \psi(x=t)=40 \mathrm{mV}$ en $x / t=1$. En general, para un tamaño de poro dado, el peifil de potencial electrostático medio para el poro cilíndrico es del orden del valor obtenido para rendija plana, donde los perfiles para la teoría de $\mathrm{PB}$ están siempre por encima de la teoría de $\mathrm{HNC} / \mathrm{MSA}$, para ambas geometrías. Para $\tau / 2=10(a / 2)$ nosotros observamos que los perfiles de $\psi(x)$ para la rendija están casi superpuestos con los del poro cilíndrico. Las diferencias más grandes se observan entre los perfiles de potencial entre las teorías HNC/MS $\ y \mathrm{~PB}$, es decir atribuidas a las correlaciones de corto alcance de los iones.

Para poros más pequeños las dos teorias dan diferencias a preciclbles, que aumentan cuando el tamaño de los poros disminuve hasta alcanzar ciertos valores, donde las diferencias disminuyen. Para la discusión sobre los coeficientes de transporte electrocinéticos, es importante mencionar que el área bajo la curva del perfil de potencial electrostático medio $\psi(x / t)$, aumenta cuando $t$ disminuye y va al valor del potencial $\zeta$ en el limite de $\tau / 2 \rightarrow a / 2$, en todos los casos.

Los materiales porosos, tales como el policarbonatc o filtres de vidrio y arenas, tienen la propiedad de "rechazar" parcialmente una solución electrolítica que pasa a través de ellas [92]. Este fenómeno es análogo al efecto Donnan, observado cn soluciones ccloidales y es la base de los procesos tecnológicos tales como la desalinación úel ag ía. El coeficiente del rechazo, $R_{+}$, es la razón de la disminución en la concentración de sal en el medio poroso, relativo al bulto en el reservorio. Este puerde ser expresado en térmircs del promedio de la concentración de coiones, en el poro $\bar{\rho}_{+}$. como 


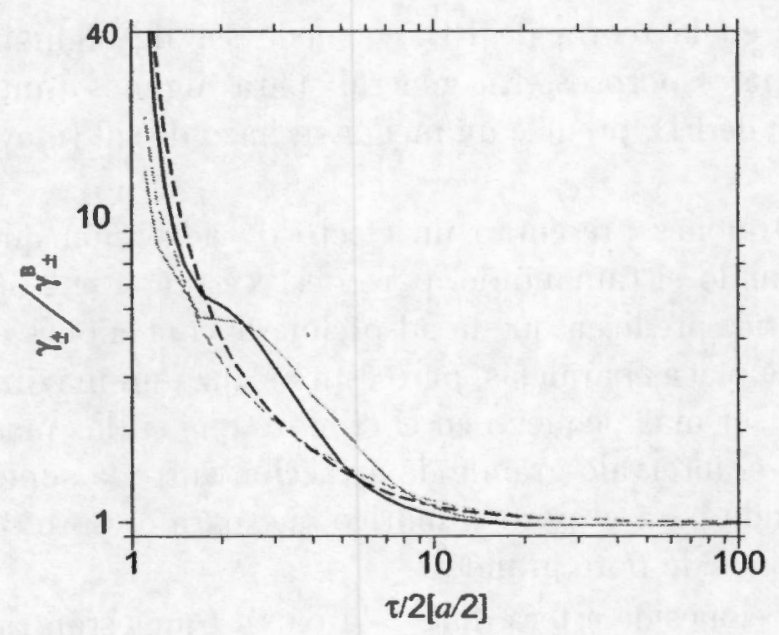

Figura 2.7: Coeficiente de actividad media $\bar{\gamma}_{ \pm}$en un poro, relativo al valor del bulto, como una función del tamaño del poro $\tau / 2$. La escala para las placas paralelas está corrida de acuerdo a la relación $\tau / 2=\frac{4}{\sqrt{3}}(h-a / 2)+a / 2$. Los parámetros y las líneas tienen la misma convención como en figura Fig.2.2.

$$
R_{+}=\frac{\rho_{+}-\bar{\rho}_{+}}{\rho_{+}}
$$

donde el promedio de la concentración de especies $i$ está definido como

$$
\bar{\rho}_{i}=\frac{2}{R^{2}} \int_{0}^{R-a / 2} \rho_{i}(r) r d r
$$

para el cilindro, y

$$
\bar{\rho}_{i}=\frac{1}{h} \int_{0}^{h-a / 2} \rho_{i}(x) d x
$$

para la rendija plana.

En la Fig. 2.3(a) nosotros comparamos el rechazo de sal en estas dos geometrías por la gráfica de $\frac{\bar{\rho}_{+}}{\rho_{+}}=1-R_{+}$como una función de la distancia media del poro, $\tau / 2$. Para un tamaño de poro dado, el rechazo de coiones es mavor en el cilindro que en el correspondiente para las placas, es decir, $\bar{\rho}_{+}$es menor para el cilindro que para las placas. También es encontrado el rechazo de sal, $\boldsymbol{R}_{+}$, es muy ineficiente para poros grandes pero éste aumenta rápidamente cuando el poro tiende a dimensiones moleculares. De acuerdo a la teoría HNC, MSA, para poros de $\tau / 2=100(a / 2)$, el rechazo de sal es sólo alrededor del $3 \%$ para las placas $y 6 \%$ para el cilindro, mientras que para $\tau / 2=10 \mathrm{n} / 2(\approx 21,25 \AA)$, esto es 10 veces mayor, cerca de alrededor del $60 \%$ para el cilíndro y $30 \%$ para la rendija plana.

El rechazo de sal es casi completo para poros aún más pequeños, pero es interesante apuntar que para poros de tamaño medio menores que $5 \AA(\tau / 2<a)$ la teoría HNC MSA predice una ligera disminución del coeficiente de rechazo iónico $\left(R_{+}\right)$. Es decir, un incremento de $\bar{\rho}_{+}$, antes de llegar a la exclusión total, cuando el poro se colapsa físicamente. Ésta 
conducta no es observada en la teoría de PB, y puede ser de alguna importancia en el cambio de iones en materiales porosos. En general, para algunos tamaños de poro y en ambas geometrías, la teoría de $\mathrm{PB}$, predice un menor rechazo de sal (mayor valor de $\bar{\rho}_{+}$) que para la teoría $\mathrm{HNC} / \mathrm{MSA}$.

Por otro lado, los contraiones presentan un efecto de adsorción que pasa a través de un máximo y va a cero cuando el tamaño del poro decrece (ver Fig.2.3(b)). Para poros de tamaño grande, ambas teorías predicen que la adsorción de contraiones es más grande en el cilindro que en la rendija de placas paralelas, pero ésta alcanza un máximo en algún tamaño de poro y entonces viene a ser más pequeño en el cilindro que en tas placas. Esto puede ser explicado por el hecho que el intervalo grande de atracción entre la superficie de la pared y los contraiones es más grande para el poro cilíndrico que para el poro de rendija plana, de aquí que dominan los tamaños de poro grandes.

Sin embargo, las interacciones de esfera dura y la pared (que están también incluidas en la teoría de $\mathrm{PB}$, por la consideración de la distancia (ip máximo acercamiento a la pared) están dominadas por tamaños de poros más pequeños, siendo también más importante en la geometría cilíndrica. Por lo tanto, el máximo de la adscrción de contraiones es más pequeño para el poro cilíndrico que para el poro de rendija de dos placas, mjentras que la teoría HNC/MSA predice valores más grandes de $\bar{\rho}_{-}$que los resuitados correspondientes por la teoría de PB. Los coeficientes cruzados de Onsager, $L_{12}=L_{21}$, que determinan la corriente producida por un gradiente de presión apiicado. o alternativamente, el flujo de volumen obtenido por un gradiente de campo eléctrico aplicado. son directamente proporcional a el flujo de volumen o coeficiente electro-ósmotico, 1 - $i$, dado por la Ec. (2.41). Por otro lado, por la Ec. (2.88), el coeficiente $\zeta_{22}$, el cual deiermina la corriente producida por la aplicación de un gradiente de campo eléctrico. es directamente proporcional al coeficiente de conductancia capilar, $1+\boldsymbol{k}$. Todas las ot ras propiedades electrocinéticas estudiadas aquí son simples funciones de estos coeficientes, como se mostró la sección anterior. Por ejemplo, la electroviscosidad depende de $(1+K) /(1-G)^{2}$ y $\mathrm{fl}$ potencial de corriente (o de flujo) es proporcional a $(1-G) /(1+k)$. En la Fig.2.t(a) se muestrà el coeficiente electro-osmótico, $1-G$, como una función del semi-tamaño riel poro, $\tau / 2$.

Se observa que el coeficiente electro-osmótico sigue una conducta opuesta a el área bajo la curva del perfil de potencial electrostático medio. $(x)$. (ver Fig. 2.2) es decir, este disminuye cuando $\tau / 2$ disminuye, yendo a cero cuando $\tau / 2$ ste aproxima a a/2. Para el poro cilíndrico, $1-G$ está bajo la curva del poro de rendija plana. mientras que para la teoría HNC/MSA da valores más grandes que la teoría de PB en ainbas geometrías. El coeficiente de conductancia capilar $1+k$ (Fig.2.4(b)) por otro lardo, es principalmente por la concentración de contraiones dentro del pero (ver $\angle .3\left(b^{\prime}\right)$. La condwet aricit eléctrica de una solución electrolítica es más grande para la geometiía cilíndrica que pará el ooro de rendija plana, sin embargo, en $\tau / 2 \approx 2(a / 2)$ éste alcanza un máximo y esta tendencia es revertida. Para poros de tamaño pequeño. las predicciones de la teoría clísica de PB , están marcadamente abajo de las curvas de la teoría HNC, MSA y ningun máximo es cbservado hasta llegar a poros de tamaño completamente pequeños, de tamaño casi cero. El coeficiente de potencial de corriente (o flujo) $F$, como una función de $\tau / 2$. se muestra en la $V i g .2 .5$. Este coeficiente rige la conducta del potencial de corriente (o Flujc). Es s. que decece para sus valores límites, $E_{s t r}^{\infty}=\frac{\varepsilon \zeta}{4 \pi \eta \sigma_{7}} P_{z}$ en $\tau / 2 \rightarrow \infty$, y a cero cuando el tawaño del poro disminuye. $F$ para el 
cilindro es más pequeño que para el poro de dos placas, para todos los tamaños de poro. Así pues, para alcanzar un potencial de corriente dado, un gradiente de presión más grande debe ser aplicado a un poro cilíndrico, en comparación a un poro de rendija plana del mismo tamaño. Como apuntamos antes [10], las correlaciones iónicas producen efectos comparables en $1-G$ y en $1+K$, que esencialmente se cancelan, cuando se toma su razón. Por tanto, $F, E_{\text {str }}$, y el potencial zeta aparente $\zeta_{\alpha}$, muestran sólo ligeras diferencias entre las teorías $\mathrm{HNC} / \mathrm{MSA}$ y $\mathrm{PB}$, en las dos geometrías.

La electroviscosidad aparente reducida tiene una conducta más compleja dado que ésta es una función de la cantidad $(1+K)(1-G)^{2}$. Sin embargo, como se muestra en la Fig. 2.6, esta conducta semeja a la adsorción de los contraiones, en la Fig.2.3. La solución electrolítica se conduce como un aparentemente fluido más viscoso en la geometría cilíndrica que en las dos placas, hasta alcanzar $\tau / 2 \approx 4(a / 2)$ donde éste alcanza un máximo y regresa hacia abajo. En general, para cada geometría, la teoría HNC/MSA predice valores más grandes de $\eta_{a}$ que los resultados correspondientes para la teoría $\mathrm{PB}$.

De la Fig.2.3 a la fig.2.6, podemos ver que las propiedades electrocinéticas para la rendija plana y para el cilindro siguen una conducta similar como una función del tamaño medio del poro $\tau / 2$. Sin embargo, las curvas para el cilindro están corridas hacia valores más grandes de $\tau / 2$, con respecto a las curvas obtenidas para la rendija plana. Como mencionamos anteriormente, es importante señalar la conexión entre los coeficientes electrocinéticos con la adsorción de coiones y contraiones. Esta conexión puede ser vista si nosotros definimos un coeficiente de actividad, $\bar{\gamma}_{i}$, en términos de las densidades promedio, $\bar{\rho}_{i}$, como $\alpha_{i}=\bar{\rho}_{i} \bar{\gamma}_{i}$; donde $a_{i}$ es la actividad dentro del poro. En equilibrio, esta actividad debe ser igual a la del bulto de la solución iónica, con concentración $\rho_{i}$ y coeficiente de actividad $\gamma_{i}^{B}$, es decir, $a_{i}=\rho_{i} \gamma_{i}^{B}=\bar{\rho}_{i} \bar{\gamma}_{i}$. Por tanto, el coeficiente de actividad medio, $\overline{\gamma_{ \pm}}$, está dado como

$$
\frac{\bar{\gamma}_{ \pm}}{\gamma_{ \pm}^{B}}=\left[\frac{\rho_{+} \rho_{-}}{\bar{\rho}_{+} \bar{\rho}_{-}}\right]^{1 / 2}
$$

Esta cantidad es trazada en la Fig. 2.7 como una función del semitamaño de los poros $\tau / 2$, para el cilindro y para la rendija plana de dos placas. Para ilustrar la equivalencia, aquí hemos usado diferentes escalas para las dos geometrías. Es decir, la escala para la rendija es recorrida de acuerdo a la relación $\tau / 2=(4 / \sqrt{3})(h-a / 2)+a / 2$, que viene de la comparación del flujo de volumen por área de sección transversal en las dos geometrías, dada por

$$
\frac{L_{11}}{\pi(R-a / 2)^{2}} P_{z}=\frac{(R-a / 2)^{2}}{8 \eta} P_{z}
$$

para el cilindro $y$

$$
\frac{L_{11}}{(h-a / 2)} P_{z}=\frac{2(h-a / 2)^{2}}{3 \eta} P_{z}
$$

para la rendija plana.

Por lo tanto, para un gradiente de presión dado, a un radio de cilindro de $\tau / 2=R$, producirá un valor similar del flujo de volumen por unidad de área de sección transversal libre, como un poro de rendija plana más pequeño de semiancho $\tau / 2=(4 / \sqrt{3})(h-a / 2)+a / 2$. Se puede observar en la Fig.2.7 que aún para tamaños de poro de alrededor de un diámetro 
iónico, la coincidencia topológica es muy estrecha, para cada teoría. Sólo para poros extremadamente pequeños, donde sólo una partícula puede coincidir o ajustarse, las correlaciones introducidas, por los efectos combinados del potencial electrostático y el volumen de exclusión, dan diferencias significativas para las dos geometrías. Fl cilindro todavía da coeficientes de actividad más grandes que los correspondientes a la rendija plana. La teoría HNC/MSA presenta una cresta en la actividad iónica que la teoría clásica de PB no predice. Referencia [93]

\subsection{Conclusiones para el potencial constante}

Hemos resuelto la ecuación HNC/MSA, y la versión de la ecuación integral de PoissonBoltzmann, para un electrolito confinado en la aproximación RPM, en dos poros de geometría diferente. Para un capilar cilíndrico delgado y para un poro de rendija plana de dimensiones similares. Las propiedades de equilibrio y los coeficientes de transporte electrocinéticos reducidos, obtenidos de la ecuación de Navier-Stokes, fueron comparacios en las dos geometrías. Los resultados numéricos muestran, como se esperaba ỵue los efectos de confinamiento con tamaño ionico finito y las correlaciones de ión-ión en el cilindrc son más notorias o están aumentadas en general, pero para poros muy pequeños ('2 y 3 radios ionicos). Sin embargo, es más notorio en el poro de dos placas este efecto, que bier podría deberse a efectos geométricos pues desde tamaños grandes de poros, se manifiesta más el efecto del tamaño de los iones en el cilindro. Las discrepancias encontradas previamente cntre las teorías de PB Y HNC/MSA, para las geometrías plana y cilíndrica, muestran ser mayores en el cilindro, en la concentración de contraiones, en la conductancia, en la viscosidad y en los coeficientes de actividad media, pues están por encima de las dos placas desde poros grandes y se invierte esta situación, sólo para poros muy pequeños. En la gráfica del flujo de volumen reducido los resultados del poro de dos placas están por encima de los resultados del poro cilíndrico, así como en el potencial de corriente. Podemos decir que existe mayor fujo de partículas en las dos placas, pues geométricamente están menos confinados que en el cilindro. Analizando los coeficientes de actividad media, un factor geométrico se cbtiene, que es particularmente válido para poros grandes.

\section{DENSIDAD DE CARGA SUPERFICIAL CONSTANTE}

En la sección anterior, analizamos las propiedades elertrocinéticus de transporte y estáticas para un electrolito $1: 1$ (monovalente), confinado por poros de geometría cilíndrica y de rendija plana (placas infinitas paralelas), como una función del tanaño del poro considerando la condición de potencial $\zeta$ con valor constante. Bajo tal cconsideración, los coeficientes de Onsager $\left(L_{m n}\right)$, son proporcionales a las funciones arlimensionales $1-G, 1+K$ y $\frac{1-G}{1+K^{*}}$. En esta parte, consideraremos un electrolito simétrico 2:2. de coṇcentración $0.5 \mathrm{M}$, en un modelo RPM, con radio de los iones de $a=4,25 \AA$, constante dielectrica $c=78,5$, temperatura $T=298 \mathrm{~K}, \mathrm{y}$ densidad de carga superficial sobre lis styerficies de las paredes interna y externa con valor constante. El ancho de la pared de ambus poros es $d=(a / 2)$, por cuestiones de simplicidad. la densidad de carga superficial solbre las paredes interior y exterior 


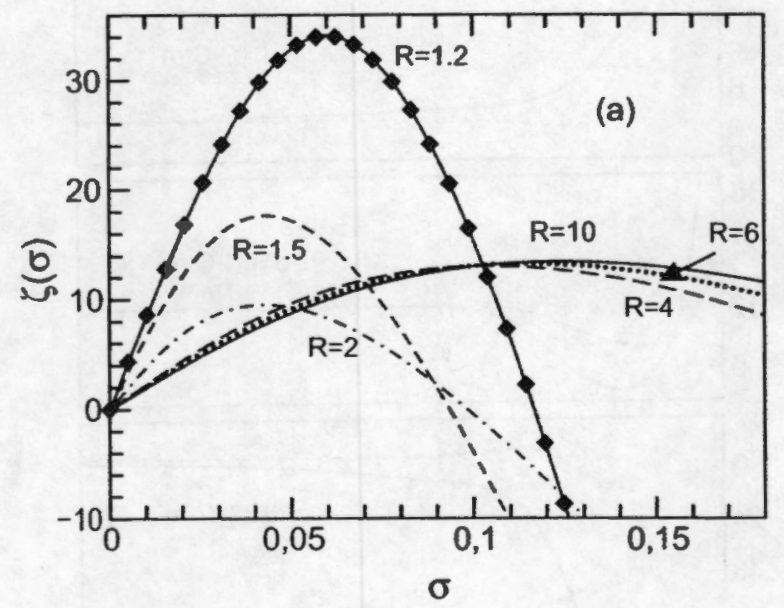

Figura 2.8: Gráficas que representan el potencial zeta como una función de la densidad de carga inducida para un poro cilíndrico (a) con el modelo de electrolito definido en la figura Fig.1, 2:2,0.5M y $\sigma_{\text {in }}=\sigma_{\text {out }}=0.272 \mathrm{C} / \mathrm{m}^{2}$

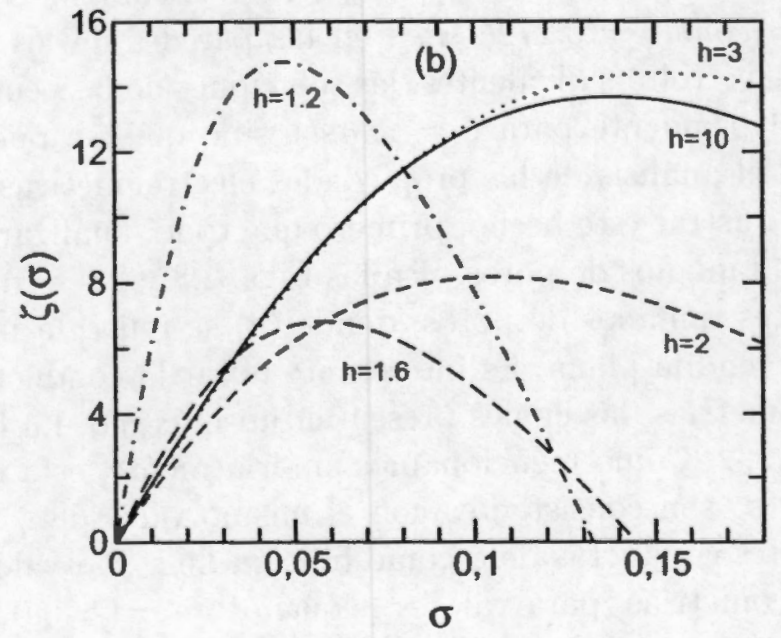

Figura 2.9: Gráficas que representan el potencial zeta como una función de la densidad de carga inducida para un poro cilíndrico (a) y un poro de dos placas (b), con el modelo de electrolito definido en la figura Fig.1, $2: 2,0.5 \mathrm{M}$ y $\sigma_{\text {in }}=\sigma_{\text {out }}=0.272 \mathrm{C} / \mathrm{m}^{2}$ 


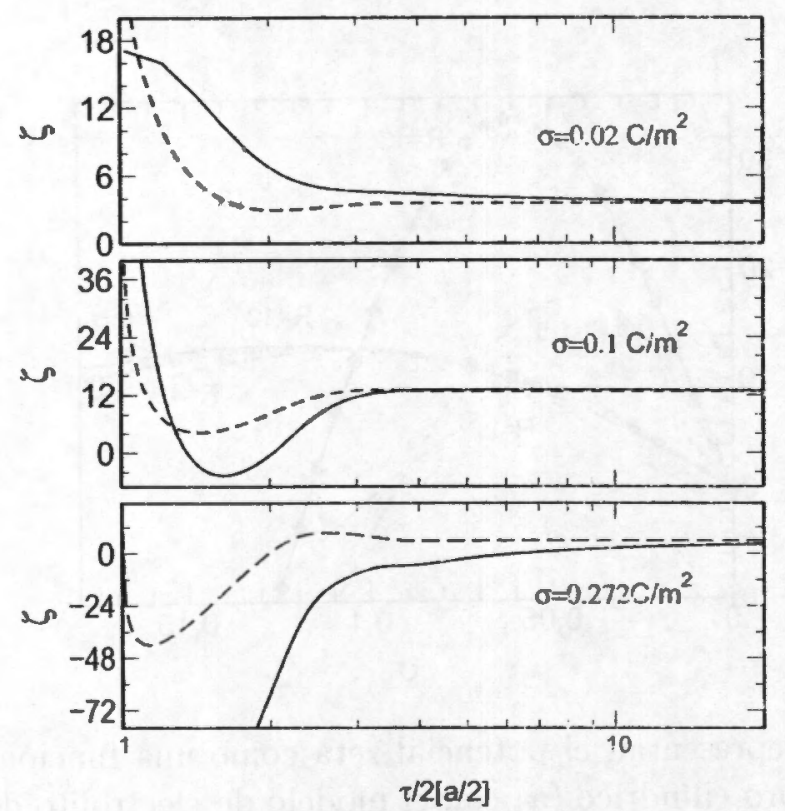

Figura 2.10: Potencial electrostático $\zeta$, como una función de $\tau / 2$, donde las líneas continuas y punteadas se refieren a las geometrías cilindrica y de rendija plana respectivamente sólo para la teoría $\mathrm{HNC} / \mathrm{MSA}$.

son tomadas de acuerdo a la condición $\sigma \equiv \sigma_{i n}=\sigma_{\text {out }}$. Hemos cambiado en esta subsección a la condición de que $\sigma=$ const. $=0,272 \mathrm{C} / \mathrm{m}^{2}$, en las paredes de los poros, en lugar de $\zeta=$ const., dado que para electrolitos divalentes, las soluciones de las ecuaciones HNC/MSA no están determinadas unívocamente para $\zeta=$ const., sino que, se pueden encontrar dos soluciones, haciendo difícil el análisis de las propiedades electrocinéticas como una función del tamaño del poro. Para ilustrar este hecho, primero que todo, analizaremos las relaciones entre $\zeta$ y $\sigma$, para diferentes tamaños de poros. En las Figs. 2.8 y 2.9 se muestra $\zeta$ como una función de $\sigma$, para diferentes tamaños de poros, donde (a) se refiere a un poro cilíndrico y (b) se refiere a un poro de rendija plana. Es interesante notar la conducta no monótona de estas curvas en las dos geometrías, las cuales presentan un máximo. La localización de este máximo es una función de $\tau / 2$. Como mencionamos anteriormente, esta conducta de $\zeta$ vs $\sigma$ implica que dos valores de $\sigma$, son consistentes con el mismo valor de $\zeta$. Sin embargo, más interesantes son las diferentes conductas de $\zeta$ como función de $\tau / 2$ a valores de $\sigma$ constante (ver fig.2.10). En ambas geometrías, para valores pequeños de $\sigma\left(\approx 0.02 \mathrm{C} / \mathrm{m}^{2}\right), \zeta$ tiende a un valor constante, para valores grandes de $\tau / 2$, y crece monótonamente cuando $\tau / 2$, decrece. Para valores intermedios de $\sigma\left(\approx 0,1 \mathrm{C} / \mathrm{m}^{2}\right)$, en ambas geometrias, $\zeta$ tiende a un valor constante a valores grandes de $\tau ; \zeta$ disminuye cuando $\tau / 2$ dismiuuve, alcanzando un mínimo y regresa a una tendencia creciente, para valores más pequeños de $\tau / 2$. En la geometría cilíndrica, el mínimo de $\zeta(\tau / 2)$ es alcanzado para valores inís grandes de $\tau / 2$ que para la geometría de rendija plana. En las dos geometrias $\zeta$ puede volverse negativa en este régimen de $\sigma$. pero en la geometría plana requiere de una densided de carga superficial más grande para que ésto ocurra. Finalmente, para densidades de carga superficial alta $\left(\sigma \approx 0,3 \mathrm{C} / \mathrm{m}^{2}\right)$. 

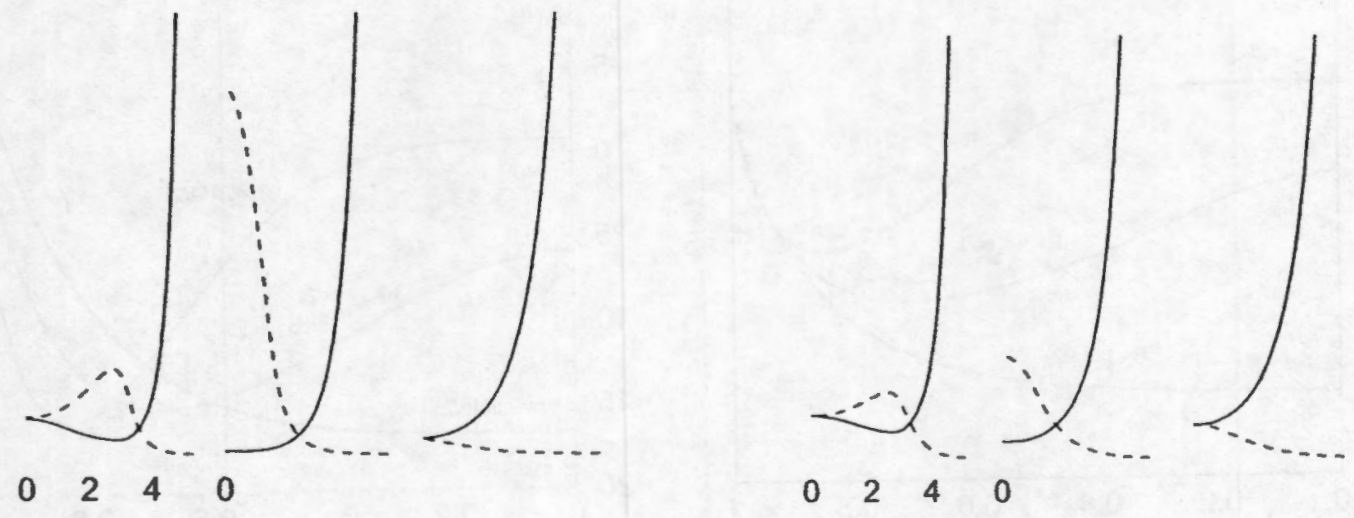

Figura 2.11: Perfil de concentración de contraiones y coiones dentro de poros cilíndrico y de placas paralelas, respectivamente de la figura izquierda a la figura derecha. Donde las líneas continuas se refieren a los contraiones y las líneas punteadas a los coiones, sólo en la teoría $\mathrm{HNC} / \mathrm{MSA}$, con las condiciones de $2: 2,0.5 \mathrm{M}$ and $\sigma_{\text {in }}=\sigma_{\text {out }}=0.272 \mathrm{C} / \mathrm{m}^{2}$.

en geometría cilíndrica $\zeta$ comienza en un valor constante para $R \rightarrow \infty$ y decrece monótonamente cuando $R$ decrece. Para valores grandes de $\sigma$, en la geometría plana $\zeta$ se conduce de manera diferente, es decir, primero sigue una tendencia creciente, cuando $R$ decrece y en algún punto se vuelve una función decreciente y finalmente vuelve otra vez a crecer. En este régimen de $\sigma, \zeta$ puede hacerse negativa en las dos geometrías si $\sigma$ es suficientemente alta, sin embargo, sigue la conducta descrita aquí. Es importante mencionar que en términos generales la conducta de $\zeta$ como una función de $\tau / 2$ será útil para el análisis de las propiedades electrocinéticas de transporte.

La conducta compleja de $\zeta$ como una función de $\tau / 2$ es el resultado de la estructura adoptada por el electrolito confinado para diferentes tamaños de poro, de aquí que nosotros analizamos la distribución de carga dentro del poro. En la Fig.2.11, mostramos los perfiles de concentración reducido (PCR) para (a) un poro cilíndrico y (b) un poro de rendija plana, y para varios tamaños de poro. En esta gráfica mostramos sólo los PCRs para $\sigma=0.272$ C $\mathrm{m}^{2}$, sin embargo, para valores más bajos de la densidad de carga superficial del poro, la conducta es cualitativamente similar, aunque menos pronunciada. En particular el fenómeno de separación de carga ocurre sólo para valores suficientemente altos de $\sigma$. Para los poros de tamaño grande los PCRs muestran la conducta típica de un electrolito divalente cerca de una pared cargada, es decir, los contraiones son atraídos mientras los coiones son expulsados lejos de la superficie. Existe una región en la cual la concentración de coiones es más alta que la concentración de contraiones, ésto se ve como una oscilación de los PCRs. En la geometría cilindrica, para el diámetro de poro de $R=3,8 a$, ocurre la así llamada segregación de carga |94|. Es decir, los coiones son segregados hacia el centro del poro, mientras los contraiones son distribuidos cerca de la pared cargada. Una conducta similar es observada para el poro de rendija plana, alrededor de $h=3,6 a$, sin embargo, no se observa ninguna segregación de 

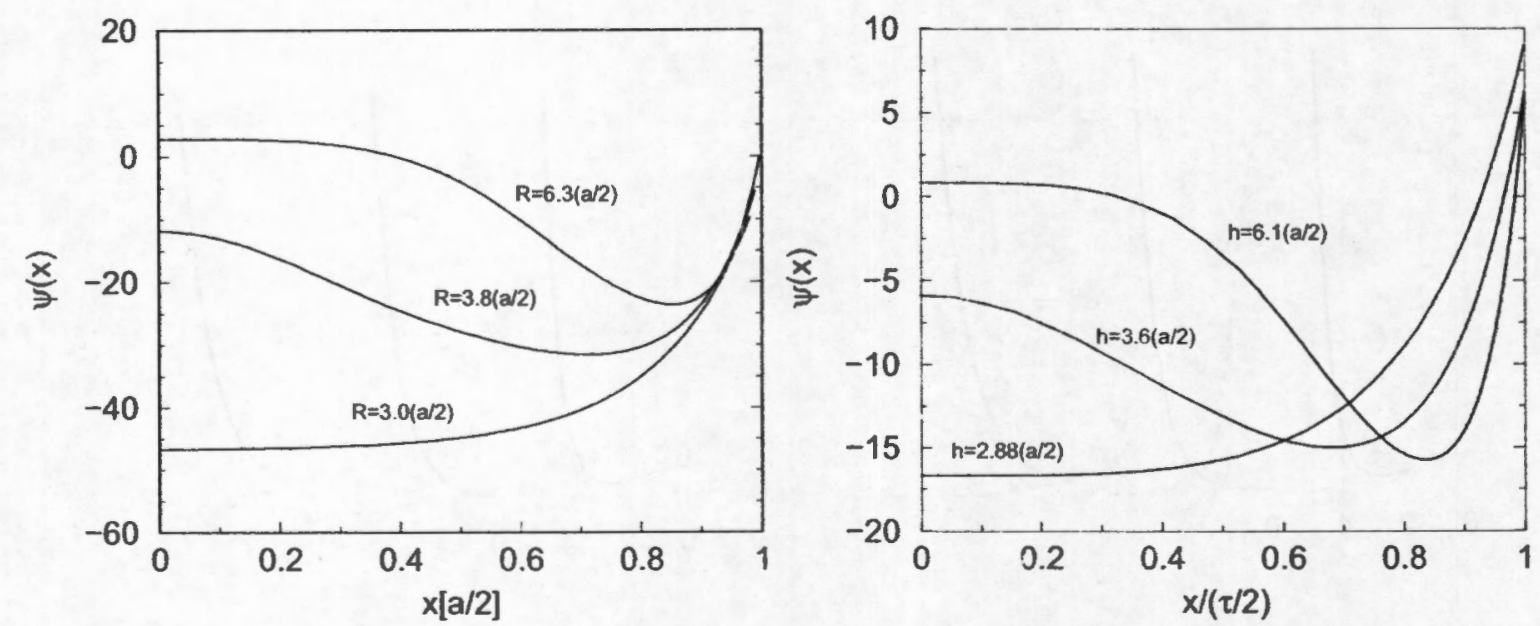

Figura 2.12: Perfil de potencial electrostático medio como función del tamaño del poro dentro de poros cilíndrico y de placas paralelas, respectivamente de izquierda a derecha, sólo para $\mathrm{HNC} / \mathrm{MSA}$, con las condiciones de $2: 2,0.5 \mathrm{M}$ 甲 $\sigma_{\text {in }}=\sigma_{\text {out }}=0.272 \mathrm{C} / \mathrm{m}^{2}$.

carga, es decir, una clara distinción entre un núcleo de sólo de coiones y una pared sólo de contraiones. Finalmente, para poros de tamaño suficientemente pequeño, la concentración de coiones disminuye en el centro del poro, y en el mismo punto, hay la misma cantidad de contraiones. Como fue apuntado anteriormente en la referencia [94], la separación de carga es debida al confinamiento de la geometría, alta valencia de las soluciones iónicas y al volumen excluido de partículas. La formación de núcleos de coiones en poros cilíndricos y esféricos está relacionada con la curvatura de las paredes de los poros, que se traduce en una muy fuerte interacción de la doble capa eléctrica sobrepuesta en poros de tamaño pequeño. De esta manera se favorece una configuración estable de coiones en el centro del poro. Todo esto está acompañado por la relación (la condición de potencial químico constante) entre el electrolito del exterior y el interior separados por la pared cargada y la necesidad del sistema de maximizar el volumen disponible [94]. En la Fig.2.12 mostramos el perfil de potencial electrostático medio dentro de los poros cilíndrico y de rendija plana, como una función de la distancia reducida a la pared cargada, que resulta del perfil de distribución de carga discutido antes en la Fig.2.10. Esto muestra los perfiles completamente diferentes dentro del poro cuando el radio del poro se reduce.

Como es lo usual, una pendiente negativa (positivo) de $\psi(x)$ implica un campo eléctrico positivo (negativo), debido a una carga neta positiva (negativa), mientras una pendiente igual a cero, implica una carga neta igual a cero. Nótese que en todos los casos, $\frac{d \psi(x)}{d x}$ va a cero cuando $x$ está alredededor del centro del poro. En primer lugar esto, es debido a la compensación exacta entre coiones y contraiones, pero también al hecho de que la carga total es igual a cero dentro de un bloque de espesor cero o cilindro de radio cero. El cambio de pendiente de cero a pendiente negativa de $\psi(x)$ (ver curvas de $R=3.8 a / 2,6.3 a / 2$ para el cilindro y $h=3.6 a / 2,6.1 a / 2$ para las placas), cuando $x$ aumıenta, está correlacionado con el hecho de que los coiones exceden en número a los contraiones dentro de esta región. 


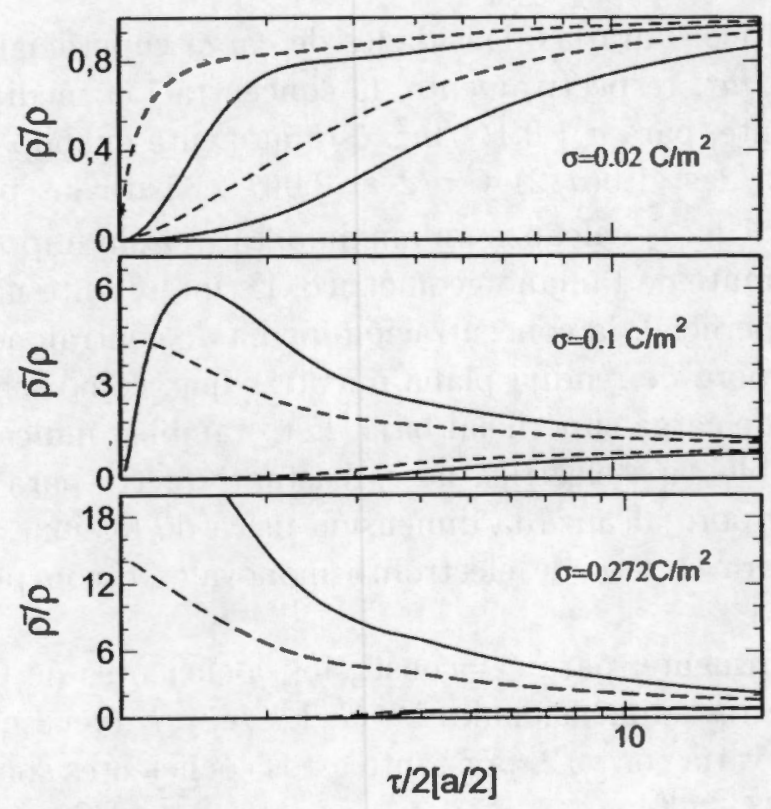

Figura 2.13: Concentración promedio reducida de contraiones y coiones como función del tamaño del poro para un cilindro y para las placas. Donde la línea continua corresponde al cilindro y la punteada a las placas. Las curvas que se encuentran por arriba de la linea continua corresponden los contraiones y las que quedan por abajo de la linea continua corresponden a los coiones.

Para las curvas con $R=3,0 a / 2$ y $h=2,88 a / 2$, podemos ver que las curvas no presentan cambio de signo de sus pendientes. Por otro lado, el cambio de pendiente de negativa a positiva, está correlacionado con el efecto opuesto, es decir, la carga neta dentro de una región es negativa dado que una cantidad más grande de contraiones es adsorvida por la superficie de los poros. Para valores pequeños de $h$ y $R$, el perfil de potencial electrostático medio es siempre monótonamente creciente, reflejando que éstas no son regiones donde los coiones exceden a los contraiones. Todos estos fenómenos, en mayor o menor grado, modulan la conducta de $\zeta$ como una función de $R$, dando un valor constante de $\sigma$, por tanto, este análisis será útil en la revisión de las propiedades de transporte electrocinéticas. En la Fig.2.13 presentamos la gráfica de la concentración media de los coiones $(+)$ y los contraiones (-), $\frac{\bar{\rho}_{i}}{\rho_{i}}$ con $i=+.-$; como una función del tamaño del poro y para tres diferentes densidades de carga superficial, es decir, $\sigma=0.02,0.1,0.272 C / m^{2}$. En ambas geometrías y para todos los regímenes de densidad de carga superficial, la concentración media de coiones es menor que en el bulto $\left(\frac{\bar{\rho}_{+}}{\rho_{+}} \leq 1 \forall \tau / 2\right)$, ésta decrece monótonamente cuando el tamaño del poro decrece. y eventualmente es igual a cero. En general, para un tamaño de poro dado, la concentración media de coiones es mayor para el poro de rendija plana que para el cilíndrico. Una conducta similar es observada para la concentración media de contraiones, para la densidad de carga superficial baja $\left(\sigma=0.02 \mathrm{C} / \mathrm{m}^{2}\right)$, es decir, decrece monótonamente $\mathbf{y} \frac{\bar{\rho}_{-}}{\rho} \leq 1 \forall \tau / 2$, sin embargo. ésta es mayor que su correspondiente concentración media de coiones, como se espera. Es importante mencionar que la concentración de coiones es mayor dentro del poro de rendija 
plana que en el poro cilíndrico. Para las densidades de carga superficial, intermedia y alta $\sigma=0.1 C / m^{2}$ y $\sigma=0.272 C / \mathrm{m}^{2}$, respectivamente, la concentración media de contraiones se comporta de manera diferente, para $\sigma=0.1 \mathrm{C} / \mathrm{m}^{2}$, ésta aumenta cuando el tamaño del poro disminuye hasta alcanzar $\tau / 2 \approx 1.0(a / 2)$ y $\tau / 2 \approx 2.0(a / 2)$ para las placas y el cilindro, respectivamente, donde se ve que $\frac{\bar{p}_{-}}{\rho_{-}}$alcanza un máximo, tal vez sin importancia porque este es un intervalo cercano al límite de tamaño geométrico. Es interesante notar que para estas dos densidades de carga superficial, la concentración media de contraiones es mayor para el poro cilíndrico que para el poro de rendija plana mientras que, como señalamos antes, ésto no es cierto para densidad de carga superficial baja. Esto también indica que la interacción debida al traslape de la doble capa eléctrica no es bastante fuerte para rechazar todos los iones cuando el tamaño del poro alcanza la dimensión física de los iones. Esta conducta es completamente diferente para el caso de electrolito monovalente con potencial superficial constante.

Como señalamos anteriormente, para $\zeta=$ const., los coeficientes de Onsager $\left(L_{m m n}\right)$ son proporcionales a los parámetros adimensionales $1-G, 1+K$, y $F$ (ver Eqs. 2.41, 2.91, 2.92), $\sin$ embargo, si $\sigma=$ const. $\zeta$ varía con $\tau / 2$, por tanto estos coeficientes son inútiles dado que, por ejemplo, $L_{12} \sim \zeta(1-G)$ y $\left(\frac{V}{I}\right)_{P_{z}=0} \sim \zeta F$. Además. $1-G$ y $F$ divergen cuando $\zeta=0$. Entonces, definimos un nuevo coeficiente con el objeto de analizar el comportamiento de $L_{12}$ como una función de $\tau$, es decir,

$$
L_{12}^{\prime} \equiv \zeta(1-G)
$$

que está dado como

$$
L_{12}^{\prime}=\zeta-\frac{2}{(R-a / 2)^{2}} \int_{0}^{R} \psi(x) x d x
$$

para la geometría cilíndrica, y

$$
L_{12}^{\prime}=\zeta-\frac{2}{h-a / 2} \int_{0}^{h} \cdot(x) d x
$$

para el poro de rendija plana. También, consideramos el potencial $\zeta$ aparente, $\zeta_{a}=\zeta F$. en lugar de únicamente $F$, cuando analizamos la razón de volumen y corriente en $P_{z}=0$, es $\operatorname{decir},\left(\frac{V}{I}\right)_{P_{x}=0^{\circ}}$

En las figs. de 2.14 a 2.16 , tenemos las gráficas de los coeficientes $L_{12}^{\prime}, 1+K \zeta_{a}$ respectivamente, como una función del tamaño del poro. Cada gráfica contiene resultados para ambos poros, cilíndrico y de rendija plana con la teoría HNC. Para $\sigma=0.02 \mathrm{C} / \mathrm{m}^{2}$ (bajo). $L_{12}^{\prime}$ se comporta similarmente al coeficiente del flujo de volumen reducido, $1-G$, para un electrolito monovalente. Es decir, para tamaños de poro suficientemente grandes de $\tau / 2$, éste alcanza un valor constante y decrece monótonamente cuando el tamaño del poro decrece.

Para tamaños del poro pequeños, es decir, cuando $\tau / 2 \rightarrow a / 2, l_{12}^{\prime}$, va a su valor límite de cero $\forall \sigma$, como ocurre con el coeficiente $1-G$. En el ralor de $\sigma=0,1 \mathrm{C} / \mathrm{m}^{2}, L_{12}^{\prime}$ aumenta lentamente cuando $\tau / 2$ disminuye, alcanzando un máximo en algún punto, en el cual empieza a decrecer. Para el poro de placas $L_{12}^{\prime}$ alcanza su máxirio en $\tau / 2 \approx 3 a / 2$, mientras para e] poro cilíndrico se alcanza en $\tau / 2 \approx 2,5 a / 2$. Es interesante observar que en el intervalo creciente de $L_{12}^{\prime}$, $\zeta$ permanece casi constante. De aquí que, semejante conducta sea debida a los valores negativos de los perfiles de potencial electrostático medio, $v(x)$ entre ciertos 


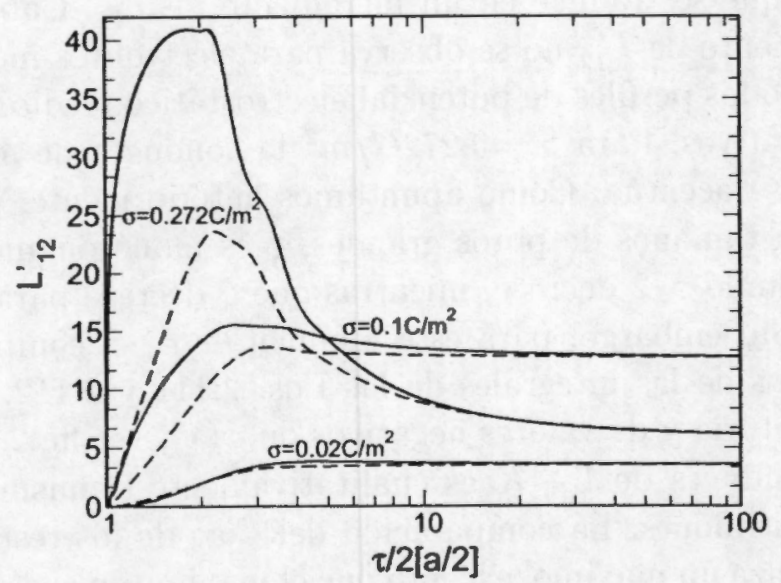

Figura 2.14: Flujo de volumen Reducido $L_{12}^{\prime}$, como una función del tamaño del poro para un cilindro (línea continua), para dos placas (línea punteada).

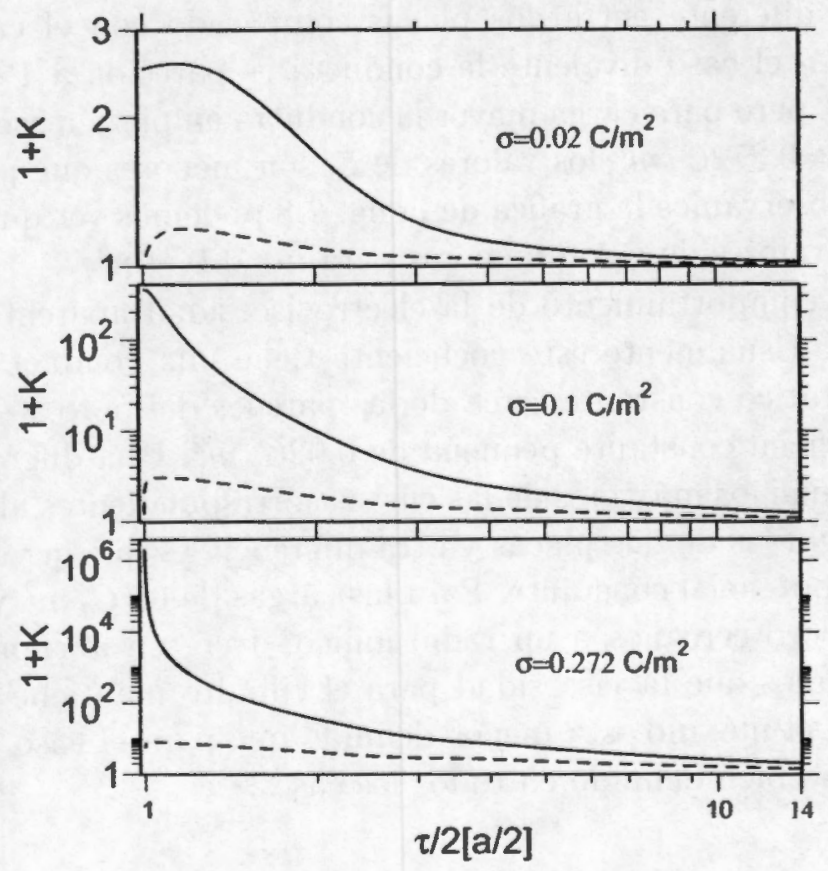

Figura 2.15: Conductancia capilar reducida $1+K$ como una función del tamaño del poro para un cilindro (línea continua) y para dos placas (línea punteada). 
valores del intervalo $[0, t]$, que a su vez causan un decremento de los términos de la integral en las Eqs. 2.111 y 2.112 que se traduce en un incremento de $L_{12}^{\prime}$. Cabe apuntar que este aumento en el comportamiento de $L_{12}^{\prime}$ no se observa para electrolitos monovalentes, debido al hecho de que en este caso los perfiles de potencial electrostático medio, $\psi(x)$, no muestran oscilaciones ni valores negativos. Para $\sigma=0.272 \mathrm{C} / \mathrm{m}^{2}$ la conducta de aumento de $L_{12}^{\prime}$. en tamaños de poro grandes se acentúa. Como apuntamos anteriormente, para esta densidad de carga superficial y para tamaños de poros grandes, $\zeta$ es monótonamente creciente para poros de rendija plana, cuando $\tau / 2$ decrece, mientras que $\zeta$ decrece para el poro cilíndrico, en este caso es negativo. Sin embargo, para este régimen de $\sigma$, el comportamiento de $l_{12}^{\prime}$ está regido por los términos de las integrales de las Eqs. 2.111 y 2.112, que son negativos debido a la conducta oscilatoria y de valores negativos de $\psi(x)$, ver fig.2.12.

Se encuentra que la conducta de $1+K$ es cualitativamente la misma que la adsorción en la fig. 2.13 para los contraiones. La comparación del caso de los resultados a potencial constante donde $1+h$ alcanza un máximo, excepto que el máximo en el caso de los resultados de densidad de carga superficial constante ocurre en tamaños de poro mucho más pequeños. Aquí se observa un aumento de conductividad de carga. Para el caso divalente todas las curvas alcanzan un valor máximo, aunque para el cilindro con carga de $\sigma=0.272 \mathrm{C} / \mathrm{m}^{2}$ parece que diverge. Para el caso monovalente la gráfica del coeficiente de potencial de corriente reducido $F$, como una función del tamaño del poro para el caso monovalente tiene una conducta casi idéntica que la del coeficiente $1-G$, porque es directamente proporcional a éste como podemos observar en la Eq.( 2.92). Pero para el caso divalente tenemos que $\zeta_{a}=\zeta F$, muestra un comportamiento muy diferente entre dos placas comparado con el caso monovalente: para $\sigma=0.02,0.1 \mathrm{C} / \mathrm{m}^{2}$, en el caso divalente la conducta es parecida a $1-G$ para el caso monovalente en el cilindro, pero para carga mayor la conducta empieza a asemejarse a las dos placas. Para el caso de $\sigma=0.272 \mathrm{C} / \mathrm{m}^{2}$ los valores de $\zeta_{a}$ son menores que para $\sigma=0.1 \mathrm{C} / \mathrm{m}^{2}$ en ambas geometrías. Si observamos la gráfica de la fig. 2.8 podemos ver que a las distancias que ocurre lo anterior los valores de $\zeta$ son menores para $\sigma=0.1 \mathrm{C} / \mathrm{m}^{2}$.

La fig.2.17 muestra el comportamiento de la electroviscosidad aparente como una función del tamaño del poro. Usualmente este coeficiente tiene una conducta similar para al caso de potencial electrostático constante cerca de las paredes del poro, y corresponde este parecido para carga superficial constante pequeña de $0.02 \mathrm{C} / \mathrm{m}^{2}$. Una diferencia importante que podemos observar, es que los máximos de las curvas correspondientes al cilindro siempre están por encima que las curvas de dos placas y otra diferencia es que la viscosidad alcanza valores más grandes para potencial constante. Para las cargas de $0.1 \mathrm{C} / \mathrm{m}^{2}$ y $0.272 \mathrm{C} / \mathrm{m}^{2}$. las curvas para tamaños de poro cercanos a un radio iónico, decrecen abruptamente. Para la carga $0.272 \mathrm{C} / \mathrm{m}^{2}$, observamos que la viscosidad para el cilindro mantiene una meseta más plana que las dos placas y el máxmo está menos definido que para el caso de dos placas en donde mantiene un máximo bien definido en todos los casos.

\subsection{Conclusiones, para carga constante}

En esta parte hemos usado la teoría HNC/MSA para el estudio de las propiedades electrocinéticas, para las dos geometrías antes mencionadas. pero para el caso divalente. La 


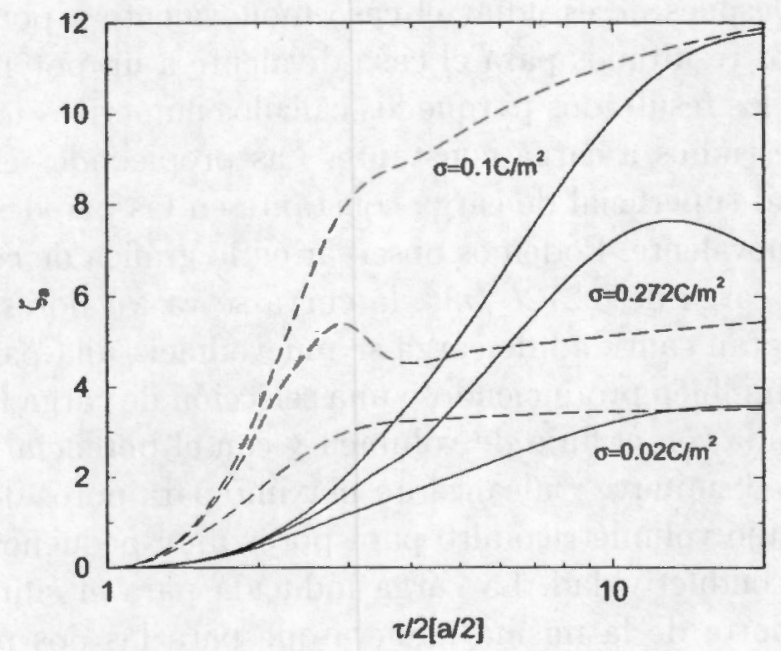

Figura 2.16: Potencial zeta aparente como una función de tamaño del poro cilindro (línea continua) y dos placas (línea punteada).
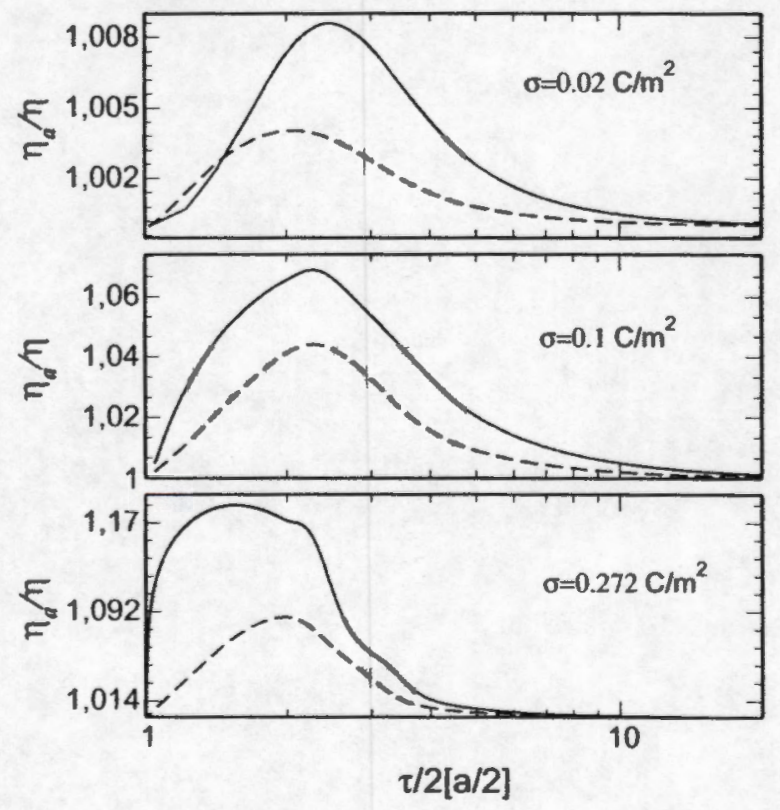

Figura 2.17: Coeficiente de viscosidad aparente reducido como una función del tamaño del poro, con las mismas condiciones fig. 2.3). 
condición adicional considerada fue haber tomado una densidad de carga superficial constante sobre las paredes. Después de estudiar el caso monovalente a potencial constante en las paredes, se buscaron los resultados para el caso divalente a un potencial zeta de $40 \mathrm{mV}$, pero no se pudieron hallar los resultados porque los cálculos numéricos no convergian, es por eso que se realizaron los estudios a carga constante. Las propiedades electrocinéticas para el caso divalente a densidad superficial de carga constante en las paredes, tienen diferencias muy marcadas al caso monovalente. Podemos observar en la gráfica de conductancia reducida (fig. 2.13), que para la carga de $0.272 \mathrm{C} / \mathrm{m}^{2}$, la curva se va a valores muy grandes de la carga, podemos decir que gran cantidad de carga se mueve hacia una parte, pero a la vez la viscosidad se hace grande también produciendose una selección de carga hacia un sentido. La variable $L_{12}^{\prime}$, está relacionada con el flujo de volumen y con el potencial zeta, ésta aumenta cuando el tamaño del poro disminuye y alcanza un máximo para poros del orden de 2 radios ionicos, ésto muestra un flujo volumétrico alto para poros muy pequeños y está de acuerdo al comportamiento de la conductividad. La carga inducida para el cilindro tiene términos logarítmicos y no se comporta de la misma manera que para las dos placas. Para el caso de $\zeta$ como función de la carga inducida podemos observar que existe en los máximos una solución única, y como el potencial $\zeta$ es importante porque se calcula experimentalmente, se podría de alguna manera caracterizar diferentes materiales de acuerdo a la carga, sobre las paredes. El potencial $\zeta$ como función del tamaño del poro para cargas superficiales grandes grandes se vuelve practicamente negativo. Para $\sigma=0,1 C / \mathrm{m}^{2}$, se observa que el potencial $\zeta$ cambia de positivo a negativo, entonces tenemos valores de la carga, donde el potencial pasa de valor positivo a valor negativo. 


\section{Capítulo 3}

\section{Interacción efectiva entre Cuatro Placas Planas Paralelas Cargadas inmersas en un Electrolito}

\subsection{Introducción}

En la naturaleza existen sustancias que han tenido múltiples aplicaciones útiles a la humanidad, como son las arcillas. Las arcillas forman parte de muchos objetos construidos por el hombre como: vasijas, edificaciones de adobe, ladrillo y cemento. También se usaron antiguamente en la escritura. La arcilla tiene propiedades plásticas, lo que significa que al humedecerla puede ser modelada fácilmente. Al secarse se torna firme y cuando se somete a altas temperaturas se producen reacciones químicas que, entre otros cambios, causan que la arcilla se convierta en un material permanentemente rígido, denominado cerámica [95]. Los distintos tipos de arcilla, cuando se mezclan con diferentes minerales y en diversas condiciones, son utilizados para producir loza. La arcilla también se utiliza en muchos procesos industriales, tales como la producción de cemento, elaboración de papel, obtención de sustancias de filtrado y en algunos procesos químicos. El cemento, es uno de los materiales de construcción más ampliamente usados en el mundo. La fuente principal de cohesión de la pasta de cemento son los hidratos de silicato de calcio (C-S-H) (que forman nanopartículas en forma de placas), y que al precipitar se apilan y unen entre sí los granos de arena (silicato tricalcico $\left.C_{3} S\right)|96|$.

Los mecanismos moleculares de la cohesión en la pasta de cemento no están bien entendidos a pesar de los tremendos esfuerzos que se han realizado para su estudio. Una razón de esto, es la carencia de datos estructurales exactos para las particulas C-S-H [96]. Sin embargo, hay el acuerdo de que es un material nanocristalino con una estructura laminar similar a un mineral llamado Tobermorita [97]. En las arcillas también intervienen procesos como el cambio, la retención y el transporte de iones [98]. Por lo tanto, el conocimiento de la estructura y dinámica del agua entre capas con iones es dẻ interés. Para el estudio de estos sistemas las simulaciones por computadora a nivel atómico han sido una herramienta útil. Los sistemas iónicos más ampliamente estudiados con este método son Na-, $\mathrm{K}$-, e hidratos de Ca-montmorillonita, dado que son los tipos de arcilla más abundantes 
$[99,100,101,102,103,104]$. Otros sistemas como son Li-, Cs-, y Sr-montmorillonita también han sido investigados $[105,106,107,108]$. La montmorillonita de Lit-saturado es el más interesante pues es considerado un prototipo de arcilla que se hincha, como lo hace el hidrato Na-montmorillonita [109].

Las arcillas minerales son aluminiosilicatos de tipo laminar con placas cargadas negativamente que se mantienen juntas por la interación con los cationes entre ellas, existen en estado natural y también se han sintetizado varios tipos en la industria. Dado que constituyen una gran porción de suelos y rocas sedimentarias, su estudio abarca muchas áreas. Las arcillas tales como el grupo de la esmectita [110] tienen la capacidad de absorver agua entre los espacios de las capas (interplacas) de la arcilla, en algunos casos producen una expansión sobresaliente del mineral. Esta expansión es primeramente de pocas capas de agua, y después con mayores contenidos de agua interlaminar [20].

El estudio de la interacción de iones con minerales de arcilla hidratados es interesante, en especial es importante entender la manera en la que los iones controlan la capacidad de ensanchamiento de la arcilla. Una forma de reducir el ensanchamiento de la arcilla es reemplazando iones de sodio por iones divalentes de calcio $[110,111,112]$. En estos sistemas los cationes de potasio reemplazan a iones como el sodio que se encuentran en los esquistos (roca que se divide con facilidad en hojas) y producen menos hidratación de la arcillas (montmorillonita)[113] que reduce el potencial de hinchado como un sistema arcilla-aguacatión $\mid 104,103,101,114,105,99,115,108,116,106,117,107,118]$. No existen muchos trabajos computacionales de hinchado de montmorillonite hidratado con cationes de potasio entre las placas $\mid 104,119,100,120,121,102,122,123]$.

Las suspensiones coloidales de arcilla aparecen en varios procesos industriales. Ejemplos de éstos son encontrados en cerámica, pinturas, cosméticos e industria petrolera $[20,124]$. Otro tipo de arcillas son los cristales minerales laminares. En este caso las particulas de Laponita son arcillas sintéticas de tres placas, compuestas de una hoja central de magnesio entre dos hojas de silicio $[125,126]$. Esta estructura de láminas en forma de placas delgadas de diámetro de alrededor de $25 \mathrm{~nm}$ y espesor de $1 \mathrm{~nm}$, puede ser separada para formar una dispersión en agua. La carga neta de las hojas de laponita se puede comportar positiva o negativamente. El tamaño de estas partículas ha permitido realizar estudios experimentales de esta arcilla sintética, con dispersiones de laponita por medio de técnicas reológicas y de dispersión de luz $[127,125,128,129]$.

Por otro lado, el hinchado en una dimensión de arcillas hidratadas ha sido muy estudiado por métodos de simulación $[99,116,115,114]$. Debido en parte a la forma altamente anisotrópica de las partículas, el potencial entre éstas es extremadamente complicado. Hay sin embargo algunos trabajos teóricos $[130,131]$. Las fuerzas de enlace que unen a las diferentes unidades estructurales (lámina más la interlámina) son más débiles que las existentes entre los iones de una misma lámina. Se ha sugerido que las correlaciones ion-ion son importantes para la estabilidad de las arcillas $[132]$ y en estudios anteriores $[133,134,135,136]$ muestran que las correlaciones pueden dar origen a fuerzas atractivas que explican la cohesión del cemento. Estudios posteriores han sido sin embargo limitantes en el tratamiento del cemento de una manera simplificada, ya que no se ha considerado que la concentración de sal es finita(28). En efecto, el sistema que produce las propiedades mecánicas de la pasta está hecho de 4 componentes: granos de cemento, nanopartículas C-S-H, la solución confinada que separa las nanopartículas y la solución acuosa que llena los poros entre los granos y que 
se refiere a una solución de bulto, la composición del bulto está determinada por el proceso de disolución que trae la colección de cationes (principalmente $\mathrm{Ca} 2+, \mathrm{K}+, \mathrm{Na}+$, etc.) y aniones (OH-, sulfato, silicatos, aluminatos, etc.), de acuerdo a la composición de la mezcla de cemento seco. Esta solución actúa como un reservorio y determina el potencial químico de todos los iones a través de la pasta incluyendo la solución confinada. Los cambios en la mezcla de los materiales pueden producir modificaciones en la composición de la solución en el bulto así como en la solución confinada y en la densidad de carga superficial de las partículas C-S-H, que afectan la cohesión de la pasta de cemento. También la composición de la mezcla cambia durante el proceso de hidratación y puede mostrar variaciones locales debido a los componentes del concreto. Entonces es importante tomar en cuenta estos efectos en un tratamiento teórico.

La hidratación y deshidratación del espacio interlaminar es también característico de otras arcillas llamadas esmectitas, cuya importancia es crucial en diferentes usos industriales. Aunque hidratación y deshidratación ocurren con independencia del tipo de catión de cambio presente, el grado de hidratación está ligado a la naturaleza del catión interlaminar y a la carga de la lámina.

La absorción de agua en el espacio interlaminar tiene como consecuencia la separación de las láminas dando lugar al hinchamiento. Este proceso depende del balance entre la atracción electrostática catión-lámina y la energía de hidratación del catión. A medida que se intercalan capas de agua y la separación entre las láminas aumenta, las fuerzas que predominan son de repulsión electrostática entre láminas, lo que contribuye a que el proceso de hinchamiento pueda llegar a disociar completamente unas láminas de otras. Cuando el catión interlaminar es el sodio, las esmectitas tienen una gran capacidad de hinchamiento, pudiendo llegar a producirse la disociación completa de cristales individuales de esmectita, teniendo como resultado un alto grado de dispersión y un máximo desarrollo de propiedades coloidales. Si por el contrario, tienen $\mathrm{Ca}$ o $\mathrm{Mg}$ como cationes de cambio, su capacidad de hinchamiento es mucho más reducida. Como puede verse, las propiedades de las arcillas son consecuencia de sus características estructurales. Por ello es imprescindible conocer la estruct ura de los filosilicatos para poder comprender sus propiedades. Las arcillas, al igual que el resto de los filosilicatos, presentan una estructura basada en el apilamiento de planos de iones oxígeno e hidróxilos, que constituyen la unidad fundamental de los filosilicatos.

En algunos filosilicatos (esmectitas, vermiculitas, micas...) las láminas no son eléctricamente neutras debido a las sustituciones de unos cationes por otros de distinta carga (sustituciones isomórficas). El balance de carga se mantiene por la presencia, en el espacio interlaminar de cationes individuales (como por ejemplo, en el grupo de las micas), cationes hidrat ados (como en las vermiculitas y esmectitas) o grupos hidroxilo coordinados. La unidad formada por una lámina más la interlámina se denomina unidad estructural. Los términos plano, capa, lámina y unidad estructural tienen significados precisos y definen partes cada vez mayores de la disposición laminar. Los cationes interlaminares más frecuentes son alcalinos ( $\mathrm{Na}$ y $\mathrm{K}$ ) o alcalinoterreos ( $\mathrm{Mg}$ y $\mathrm{Ca}$ ). Así pues, la existencia de carga en las láminas se compensa. con la entrada en el espacio interlaminar de cationes débilmente ligados con estado variable de hidratación [137], que pueden ser intercambiados fácilmente mediante la puest a en contacto de la arcilla con una solución saturada en otros cationes, a esta propiedad se le conoce como capacidad de intercambio catiónico y es también la base de multitud de aplicaciones industriales de las arcillas. Algunas arcillas encuentran su principal campo de 
aplicación en el sector de los absorbentes ya que pueden absorber agua u otras moléculas en el espacio interlaminar (esmectitas) o en los canales estructurales (sepiolita y paligorskita).

La mayor parte de las propiedades físico-químicas de las arcillas derivan de su morfología laminar (filosilicatos) y del tamaño pequeño de la partícula (inferior a 2 micras), así como de las sustituciones isomórficas en las láminas que dan lugar a la aparición de carga en las mismas. Los dos primeros factores conjugados producen, por una parte, un valor elevado de la superficie específica de estos materiales, y a la vez, la presencia de una gran cantidad de superficie activa, con enlaces no saturados. Por ello pueden interaccionar con muy diversas sustancias, en especial compuestos polares, entre los que destaca el más importante: el agua. Las consecuencias inmediatas son el comportamiento plástico en mezclas arcilla-agua con elevada proporción sólido/líquido y el hinchamiento o "swelling" con el desarrollo de propiedades reológicas en suspensiones acuosas.

Es esencial entender que las partículas de arcilla no son por sí mismas partículas nanométricas, sino que están conformadas por una estructura molecular basada en el apilamiento de láminas, cuyo espesor mide aproximadamente $1 \mathrm{~nm}$, mientras que sus dimensiones laterales pueden variar desde $30 \mathrm{~nm}$ hasta varias micras.

Las arcillas minerales, también se conocen como filosilicatos o silicatos laminares. Los filosilicatos pertenecen fundamentalmente a cuatro grupos principales: caolinita, esmectita. illitas y clorita [138]. En su aspecto primario, las partículas de arcilla se componen de la asociación de varias láminas apiladas, las cuales forman agregados irregulares,(refer 15,16) tal como se presenta en la figura 3.1. A gran escala, cada lámina posee una elevada relación de aspecto, alrededor de 100 a $200 \mathrm{~nm}$ de longitud y $1 \mathrm{~nm}$ de espesor. A la unidad formada por la unión de capas tetraédricas y octaédricas se le denomina lámina y tiene un espesor de $7 \AA$ y de $9 \AA$ aproximadamente. Así, las láminas de las arcillas se originan por el apilamiento de planos alternos de iones $\left(\mathrm{O}^{-}\right.$y $\left.\mathrm{OH}^{-}\right)$y cationes $(\mathrm{Si} 4+, \mathrm{Mg} 2+$ y $\mathrm{Al} 3+$ ). El espacio interlaminar, es el espacio existente entre dos láminas consecutivas, también conocido como galería. Resumiendo, las arcillas presentan formas apiladas, cuyas geometrías las podemos representar aproximadamente como estructuras autoensambladas en forma de planos laminares. Físicamente se considera un coloide $[139,20,140,141,142,143]$.

El diámetro de las partículas de arcilla es inferior a $2 \mu \mathrm{m}$. Estos sistemas pueden modelarse a través de placas cargadas. Estas partículas pueden estudiarse mediante un modelo representado en la figura 3.1. Por otro lado, experimentos de mezcla de polielectrolitos de DNA con liposomas cationicos dan lugar a un autoensamblamiento multilaminar de membranas de liposomas cargadas positivamente intercaladas con polielectrolitos de DNA negativos [144].

En teoría de líquidos y a niveles nanométricos, el confinamiento entre paredes afecta las fuerzas electrostáticas de muchos cuerpos, las correlaciones de corto alcance, así como también las interacciones de la doble capa de las paredes y las partículas como macroiones. Los efectos del confinamiento aparecen en un amplio intervalo de escalas espaciales. Estos efectos se observan a escalas muy grandes [145], hasta escalas subatómicas [146|: pasando por las escalas moleculares $[147,148]$. Al confinar partículas a nivel molecular entre paredes [149], es posible inducir un cierto ordenamiento, también pueden producirse fuctuaciones de densidad, provocar cambios dieléctricos en líquidos e incluso mejorar la miscibilidad en mezclas de polímeros $|148,147,150|$. Otros experimentos muestran fenómenos asociados con macroiones cargados en una solución acuosa, confinados entre dos placas planas paralelas cargadas $|151,152,153|$ (con los macroiones y las placas de carga similar), se induce una 


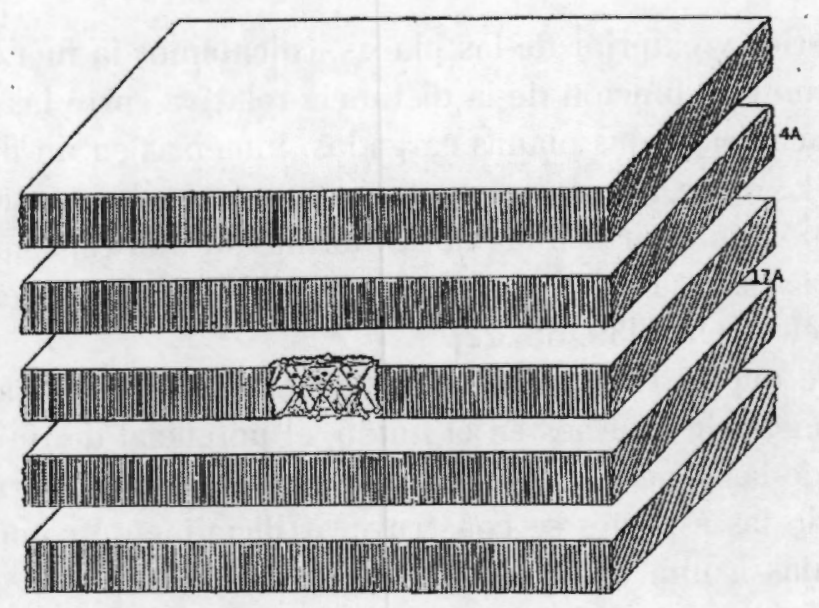

Figura 3.1: Modelo de una partícula de arcilla

atracción entre la pared y los macroiones [154], también los macroiones exhiben una atracción entre ellos mismos $[155,156]$. En sistemas coloidales se sabe que estos arreglos producen atracciones que todavía no son explicadas [157] y en la biología la presencia de formas de bicapas de lípidos cargadas en forma de placas planas paralelas muestra que se induce una atraccción entre las moléculas cargadas de DNA $[158,144,159,160,161]$. En una mezcla de polielectrolitos con liposomas cationicas estas fuerzas dan origen a autoensamblamiento multilaminar de membranas de liposomas cargadas positivamente e intercaladas de polielectrolitos DNA negativos [144].

Estudios de un electrolito confinado entre superficies cargadas han sidos considerados teóricamente desde los trabajos pioneros de Derjaguin, Landau, Verwey Overbeek (DLVO) $[5,162,3]$. En su trabajo ellos resuelven la ecuación de Poisson- Boltzmann para la doble capa eléctrica (EDL) producida por un electrolito confinado entre las superficies cargadas. Dado que la aproximación anterior desprecia las correlaciones iónicas, la teoría DLVO puede considerarse válida, sólo para un régimen restringido de condiciones, es decir para soluciones monovalentes de baja concentración y bajo potencial superficial electrostático. Este sistema ha sido ampliamente estudiado por medio de teorías más refinadas de fluidos $\mid 79,80,53,26$, $35,163]$, que incorporan correlaciones iónicas de corto alcance en la EDL.

Estudios de la fuerza de interacción entre dos coloides de forma de placas cargadas han mostrado que electrolitos multivalentes a concentraciones altas y moderadas producen un decaimiento no-monótono que puede volverse atractivo en ciertas distancias de separación $\{22,79,80\}$. Debe señalarse que el estudio de fluidos cargados y confinados lleva implícita una gran complejidad ya que se combinan las interacciones de largo alcance, los efectos resultantes de muchos cuerpos y los efectos debidos a las interacciones de corto alcance. Un modelo para estudiar estos sistemas consiste en colocar paredes planas superpuestas en presencia de un fluido cargado |164].

En este capítulo aplicamos el formalismo de ecuaciones integrales para el estudio de la interacción efectiva entre cuatro placas paralelas cargadas en un electrolito a través de la teoría HNC/MSA. Esto es, partiendo del cálculo de las funciones de distribución en equilibrio 
de un electrolito en el interior y exterior de las placas, calculamos la fuerza promedio sobre cada una de las placas como una función de la distancia relativa entre las cuatro placas.

Para el caso de dos placas paralelas planas cargadas, inmersas en un fluido iónico, se ha estudiado con esta teoría. Es decir, las placas se consideran como dos partículas de la misma especie que están unidas por una barra imaginaria, formando una especie de "mancuerna". Ha sido aplicada esta teoría exitosamente para calcular la fuerza entre dos placas paralelas cargadas, inmersas en un electrolito $[39,38,22]$.

Partiendo del hecho de que la teoría que hemos mencionado en el capítulo 2, no está restringida respecto al número de especies en el fluido, el potencial de interacción entre las partículas o la geometría de las especies; se puede pensar en un fluido formado por $(n+1)$ especies, en el cual, una de las especies se construye artificialmente como una mancuerna formada por dos placas fijas a una distancia dada entre sus centros, $\tau \equiv\left|\mathbf{r}_{A}-\mathbf{r}_{B}\right|$ (ver Fig.2.1a). Si llamamos a la especie artificial $\alpha$ y tomamos el límite $\rho_{\alpha} \rightarrow 0$, la ecuación de Ornstein-Zernike para la función de correlación entre la especie $\alpha$ e $i$ es expresada como vimos en el capítulo 2, $g_{a i}\left(\mathbf{r}_{21}\right)$ de la especie $i$ alrededor de la partícula $\alpha$

Si consideramos un problema similar al caso anterior, donde el sistema consta de cuatro placas planas paralelas infinitas cargadas e inmersas en un electrolito; quisieramos calcular la función de distribución de las partículas que se encuentran cerca de las placas en el fluido. Si una partícula interactúa con otra, donde la interacción de las "dos partículas" es tal que la primera particula es una partícula del fluido iónico y la segunda "partícula" es artificial (de la forma como mencionamos anteriormente) y consiste de una doble mancuerna unida por tres barras imaginarias (equivalente a cuatro placas colocadas en forma paralela y separadas a una distancia fija), esta parte se estudia en la siguiente sección. Una de las variables más importantes que queremos calcular es la presión neta sobre cada una de las placas. Esta presión está definida como la presión de un lado de la placa, menos la presión del otro lado de la misma placa [22]. Los perfiles de concentración de los iones dentro y fuera de las placas nos permiten calcular el potencial de interacción de la fuerza media sobra cada una de las placas. En este capítulo continuamos usando la teoría de fluidos inhomogéneos de $\left.\mathrm{HNC}_{/} \mathrm{MSA} \mid 21,23,165,166,167,168\right]$ y el modelo primitivo restringido (MRP) para la solución iónica $\mid 37,40,4,167,169]$. Para la aproximación (MSA) ver algunos trabajos como, $[170,171,40,172,173,174,175,176,177,35]), y$ para la aproximación (HNC), ver $[40,178,37,46]$

La presión es calculada por un método convencional [80], mostrado más adelante. Presentaremos el desarrollo matemático de la ecuación de HNC/MSA, para los perfiles de concentración iónica de la doble capa eléctrica de las cuatro placas. Otro estudio reslizado en esta tesis es la resolución numérica de estas ecuaciones para electrolitos simétricos monovalentes $(1: 1)$ y divalentes $(2: 2)$, con diferentes concentraciones y potenciales eléctricos fijos sobre las placas. Hay que aclarar que este sistema presenta otro grado de dificultad y mayor complejidad que el de dos placas. Este desarrollo puede considerarse como una extensión de la teoría de dos placas (TPE-HNC/MSA $\mid 79,80,179])$, y a la vez esta teoría puede reducirse al problema de dos placas cargadas o de una placa cargada $[37,40]$.

Este capítulo está organizado de la siguiente manera: En la sección 3.2 aplicamos el método usado en TPE-HNC/MSA |79, 80], y derivamos las ecuaciones para los perfiles de concentración. En la sección 3.3, se describe y estudia el modelo particular que hemos abordado. En la sección 3.4 se deduce como se calculan las cargas inducidas en el espacio por 
las placas y en la sección 3.5 terminamos la descripción teórica de este capítulo indicando como obtenemos las fuerzas y las presiones sobre las placas.

Los resultados numéricos son mostrados y discutidos en la sección 3.6. En la sección 3.7 presentamos las conclusiones. Al final de este capítulo se anexan varios apéndices: En el apéndice A se muestran las expresiones explícitas para los kernels de la ecuación HNC/MSA. En el apéndice B las ecuaciones analíticas son desarrolladas para el caso de cuatro placas. En el apéndice $C$, se muestra la fuerza neta en las placas. En el apéndice D se muestran las expresiones para calcular el potencial electrostático en las diversas regiones dentro y fuera de las cuatro placas. El apéndice $\mathrm{E}$ está relacionado con la presión osmótica. El apéndice $\mathrm{F}$ nos muestra de manera resumida la forma de resolver el problema numérico. El útimo apéndice, muestra algunas figuras de gráficas que se relacionan con los resultados.

\subsection{Teoría de Funciones de Distribución}

La ecuación integral de Ornstein-Zernike (OZ) para un fluido con $(\mathrm{N}+1)$ componentes, usando el método directo está dada por $[23,37,40]$,

$$
h_{i j}\left(\mathbf{r}_{21}\right)=c_{i j}\left(\mathbf{r}_{21}\right)+\sum_{m=1}^{N+1} \rho_{m} \int h_{i m}\left(\mathbf{r}_{23}\right) c_{m j}\left(\mathbf{r}_{13}\right) d v_{3} .
$$

donde $c_{i j}\left(\mathbf{r}_{21}\right)$ es la función de correlación directa y $h_{i j}\left(\mathbf{r}_{21}\right)=g_{i j}\left(\mathbf{r}_{21}\right)-1$ es la función de correlación total entre dos partículas de especies $i$ y $j$ con posiciones relativas $\mathbf{r}_{21}=\mathbf{r}_{2}-\mathbf{r}_{1}$; $g_{i j}\left(\mathbf{r}_{21}\right)$ es la función de correlación por pares o función de distribución radial (en nuestro caso el perfil de concentración de iones), $\rho_{m}$ es la concentración en el bulto de las especies $m$ e $i, j=1, \ldots, N+1$. Como en la sección anterior, considerando al poro como una especie más en el fluido, definimos la especie $N+1=\alpha, \mathrm{y}$ además, con una consideración adicional de que esta especie se encuentra muy diluida en todo el espacio $\left(\rho_{a} \rightarrow 0\right)$ de tal manera que

$$
h_{\alpha j}\left(\mathbf{r}_{21}\right)=c_{\alpha j}\left(\mathbf{r}_{21}\right)+\sum_{m=1}^{N} \rho_{m} \int h_{\alpha m n}\left(\mathbf{r}_{23}\right) c_{m j}\left(\mathbf{r}_{13}\right) d v_{3}
$$

Sustituyendo la aproximación (HNC) en la función de correlación directa entre el poro y el fluido, $c_{\alpha j}\left(\mathbf{r}_{21}\right)$, es decir,

$$
c_{\alpha j}\left(\mathbf{r}_{21}\right)=h_{\alpha j}\left(\mathbf{r}_{21}\right)-\beta u_{\alpha j}\left(\mathbf{r}_{21}\right)-\ln g_{\alpha j}\left(\mathbf{r}_{21}\right) .
$$

donde $u_{\alpha i}\left(\mathbf{r}_{21}\right)$ es el potencial de interacción directa entre el poro y una partícula de especie $j, \beta=1 / k_{B} T, k_{B}$ es la constante de Boltzmann y $T$ la temperatura absoluta. La función de correlación directa entre dos partículas del fluido, $c_{m j}\left(r_{13}\right)$, es sustituida por la aproximación esférica media (MSA) para un electrolito RPM dada como

$$
c_{m j}^{N S A}\left(r_{13}\right)= \begin{cases}c_{s}\left(r_{13}\right)+z_{i} z_{m 2} c_{d}^{s r}\left(r_{13}\right)-\frac{z_{m z_{j} \beta \epsilon_{t}^{2}}}{\epsilon r_{13}}, & \text { for } r_{13}<a \\ -\frac{z_{m z_{j} \beta e^{2}}}{\epsilon r_{13}} & \text { for }\left|r_{13}\right| \geq a\end{cases}
$$

siendo $c_{s}\left(r_{13}\right)$ y $c_{d}^{s r}\left(r_{13}\right)$ funciones de corto alcance dadas en el apéndice. Así pues, sustituvendo las ecs. (3.3) y (3.4) en la Eq. (3.2) nos queda 


$$
\begin{aligned}
g_{a j}\left(\mathbf{r}_{21}\right) & =\exp \left\{-\beta u_{\alpha j}\left(\mathbf{r}_{21}\right)+\sum_{m=1}^{N} \rho_{m} \int h_{\alpha m}\left(\mathbf{r}_{23}\right) c_{m j}^{M S A}\left(\mathbf{r}_{13}\right) d v_{3}\right\} \\
& =\exp \left\{-\beta u_{a j}\left(\mathbf{r}_{21}\right)-\frac{z_{j} \beta e^{2}}{\epsilon} \int \rho_{\alpha d}\left(\mathbf{r}_{23}\right) \frac{d v_{3}}{r_{13}}\right. \\
& \left.+\frac{z_{j} \beta e^{2}}{\epsilon} \int \rho_{\alpha d}\left(\mathbf{r}_{23}\right) c_{d}^{s r}\left(r_{13}\right) d v_{3}+\int \rho_{\alpha s}\left(\mathbf{r}_{23}\right) c_{s}\left(r_{13}\right) d v_{3}\right\}
\end{aligned}
$$

donde hemos definido

$$
\begin{gathered}
\rho_{\text {oul }}\left(\mathbf{r}_{13}\right)=\sum_{m=1}^{N} z_{m} \rho_{m m} h_{\alpha m u}\left(\mathbf{r}_{13}\right) \\
\rho_{\alpha s}\left(\mathbf{r}_{13}\right)=\sum_{m=1}^{N} \rho_{m} h_{\alpha m}\left(\mathbf{r}_{13}\right)
\end{gathered}
$$

Las ecs. (3.5) han sido derivadas para el caso general y se refieren a las ecuaciones integrales HNC/MSA, para partículas de especie $j$ cerca de la partícula de especie $a$. Más adelante aplicaremos la ec. (3.5) a un electrolito MPR confinado entre las cuatro placas.

En resumen, consideremos que el sistema consta de 3 especies, dos de las cuales son las especies $j=1,2$ (componentes del electrolito) y la otra es la especie a. Esta última consta de una partícula que está formada por cuatro placas paralelas cargadas de extensión infinita, separadas por una distancia entre ellas, $y$ a dilución infinita, i.e., $\rho_{a}$ tiende a cero. Las especies $j$ son esferas duras de diámetro $a$, con la carga en sus centros. Entonces, para conocer la función de correlación total, $h_{a j}\left(\mathbf{r}_{12}\right)$, entre la especie a y una partícula de especie $j$ tenemos que

$$
h_{\alpha j}\left(\mathbf{r}_{12}\right)=c_{\alpha j}\left(\mathbf{r}_{12}\right)+\sum_{m=1}^{2} \rho_{m i} \int c_{m j}\left(\mathbf{r}_{13}\right) h_{a m n}\left(\mathbf{r}_{23}\right) d v_{3}
$$

en donde la suma no toma en cuenta a la partícula de especie $\alpha$ va que $\rho_{a} l p h a=0 \mathrm{y}$, por lo tanto, $j=1,2, \ldots, N$ (en este caso $N=2$ son los cationes (+) y anjones (-).

\subsection{El modelo específico}

El sistema que estudiaremos es: Un poro de rendija plana, formado por cuatro placas infinitas paralelas, cargadas, con una distancia de separación entre el eje de simetría y las superficies internas de las placas interiores de $t_{1}$ y una distancia entre las superficies externas de las placas interiores y la superficies internas de las placas exteriores de $t_{2}$ (ver Fig. 3.2).

Las paredes de las placas tienen densidad de carga superficial uniforme $\sigma_{1}, \sigma_{2}$ y $\sigma_{3}, \sigma_{4}$ iguales como se muestra en la Fig. 3.2, tal que $\sigma \equiv \sigma_{1}=\sigma_{2}=\sigma_{3}=\sigma_{4}$. Es importante hacer notar que el problema tiene simetría con respecto al plano en el centro del sistema, por lo que la parte derecha del sistema, es igual a la parte izquierda del sistema. Se denominará a las placas que encuentran cerca del eje de simetría, placas internas o placas 1, y a las otras, placas, placas externas o placas 2.

Las placas se encuentran inmersas en un electrolito, $y$ las especies a corresponden a las cuatro placas, con un modelo primitivo restringido (MPR), de esferas duras cargadas de 


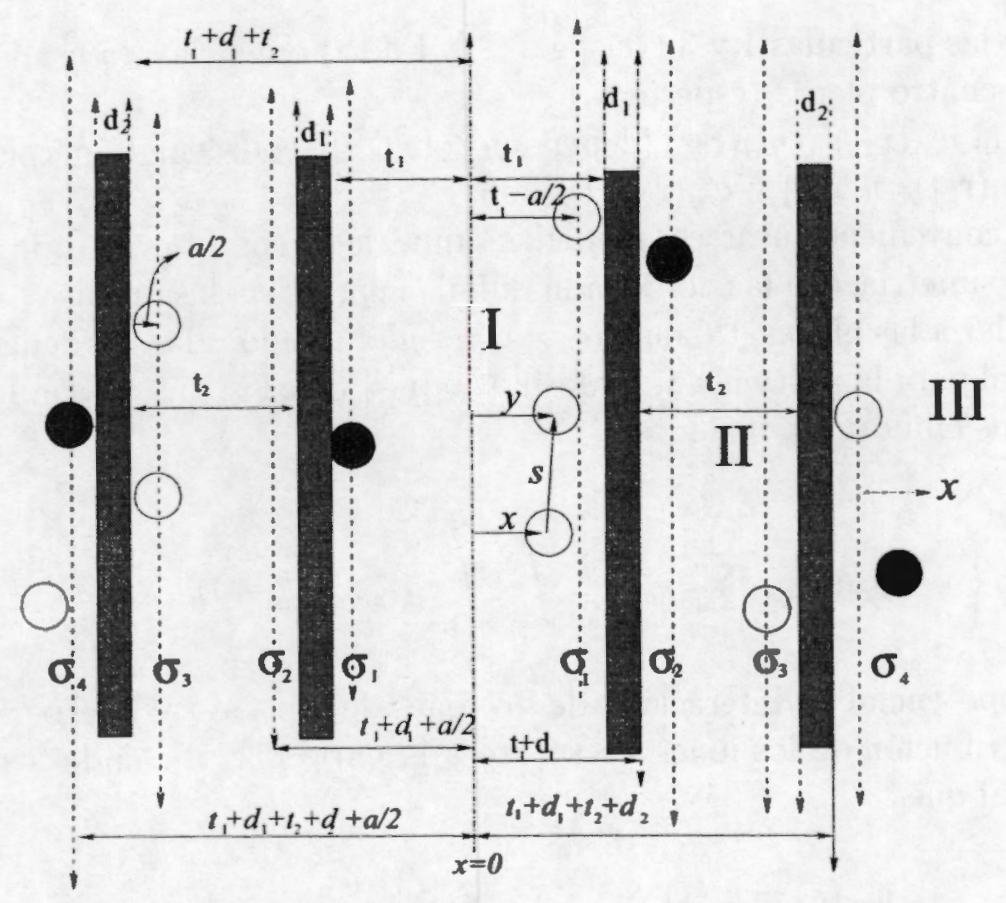

Figura 3.2: Representación geométrica para el sistema de cuatro placas cargadas

diámetro a y con cargas $z_{+} e$ y $z_{-} e$, ubicadas en el centro de las esferas, siendo $z_{-}$y $z_{+}$ las valencias de los aniones y cationes, respectivamente. La energía potencial de interacción entre dos partículas de especie $i$ y $m$, separadas por una distancia $r_{13}$, es

$$
u_{i m}\left(r_{13}\right)= \begin{cases}\frac{\frac{z_{i} z_{m} e^{2}}{\epsilon r_{13}}}{\epsilon} & \text { for } r_{13} \geq a \\ \infty & \text { for } r_{13}<a\end{cases}
$$

Una condición importante que debe satisfacer el fluido es ser electroneutro lejos de la pared (en el bulto), es decir,

$$
\sum_{m=1}^{n} z_{m} \rho_{m}=0
$$

donde $\rho_{m}$ es la concentración en el bulto de la especie $m$.

Las posiciones de los iones están referidas respecto a sus centros, entonces la posición más cercana de un ion a cualesquiera de las superficies de las paredes de las placas es un radio iónico, también conocida como distancia de máximo acercamiento a la pared. En la región entre el centro y la placa 1 , el valor de su coordenada en $x\left( \pm\left(t_{1}-a / 2\right)\right)$. La coordenada en la posición entre la región entre la placa 1 y la placa 2 , a la distancia de máximo acercamiento con la placa 1 , es $t_{1}+d_{1}+a / 2$ y cuando se encuentra a la distancia de máximo acercamiento con la placa 2 es $t_{1}+d_{1}+t_{2}-a / 2$. El ancho de todas las placas es $d=d_{1}=d_{2}$. En cuanto a las distancias, perpendiculares al eje de simetría de las placas, tenemos la distancias del eje de simetría a las partículas 1 y 3 que las representamos con las letras $x$ e $y$, respectivamente, $s$ 
es la distancia entre las partículas 1 y 3 (ver fig. 3.2). En le nomenclatura useda la partícula 2 corresponde a las cuatro placas (especie $\alpha$. )

En esta geometría $u_{\alpha i}\left(\mathbf{r}_{21}\right)$ y $\boldsymbol{g}_{\alpha i}\left(\mathbf{r}_{21}\right)$, depencien sólo de lá coordenada perpendicular a la pared, $x$, tal que $u_{\alpha i}\left(\mathbf{r}_{21}\right)=u_{\alpha i}(x)$ y $g_{\alpha i}\left(\mathbf{r}_{21}\right)=g_{\alpha i}(x)$.

En este caso es conveniente usar coordenades cilíndricas, con ol origere de coordenadas centrado en el eje de simetría, con la coordenada radial $r$, parálela z las placas y la coordenada axial, $y$, perpendicular a las placas, tal que $d v_{3}=r d r c^{\prime} y d \phi$, siendo $\zeta$ la coordenada angular y $x$ la coordenada axial para la partícula 1, es decir $x=\left|r_{21}\right|$ (ver Fig. 3.2. Usan do la ecuación (3.5), en coordenadas cilíndricas, tenemos

$$
g_{\alpha i}(x)=\exp \left\{-\beta u_{\alpha i}(x)+\sum_{m=1}^{2} \rho_{m} \int_{-\infty}^{\infty} \int_{0}^{\infty} \int_{0}^{2 \pi} c_{i m}(s) h_{\infty m} ;\right) d \phi r d r d z ?
$$

donde $u_{a i}\left(\mathbf{r}_{21}\right)$ es el potencial de interacción de los iones de especie $i$. la cspecip o y $g_{a i}(x)$ es la función de distribución de los iones $i$ respecto a la partículi a. Donde hemos definido $\mathbf{r}_{13}=s, y \mathbf{r}_{23}=y$, tal que

$$
c_{i m}(s)=c_{s}(s)+z_{i} z_{m} c_{d}^{m}(s)+\beta e^{2} / \varepsilon \varepsilon
$$

con

$$
s^{2}=r^{2}+x^{2}+y^{2}-2 x y
$$

donde $r d r=s d s$.

El potencial de interacción $u_{\alpha i}(x)$, puede descomponerse como sigue

$$
u_{\alpha j}(x)=u_{a i}^{*}(x)+u_{a, x i}^{e l}(x)
$$

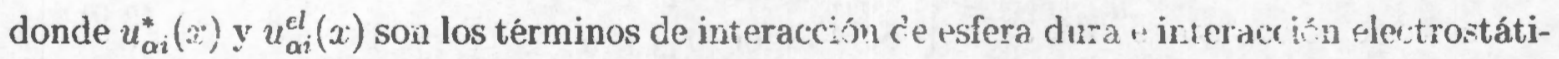
ca, respectivamente, que expresamos como

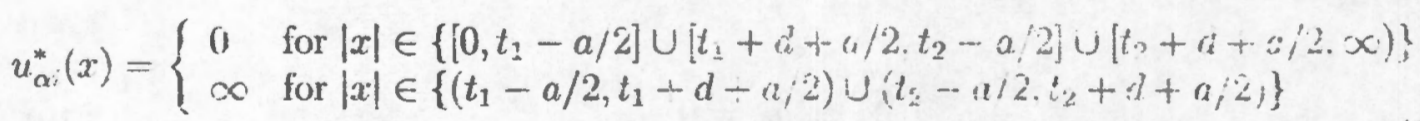

y

$$
\begin{aligned}
u_{o i}^{c l}(x) & =-\frac{2 \pi e z_{i}}{\varepsilon}\left[\left(x+t_{1}+d_{1}+t_{2}+d_{2}-\left|x-t_{1}-d_{1}-t_{2}-d_{2}\right|\right) \sigma\right. \\
& +\left(x+t_{1}+d_{1}+t_{2}-\left|x-t_{1}-d_{1}-t_{2}\right|\right) \sigma_{3}+\left(x+t_{1}+d_{2}-\left|\cdots-t_{1}-d_{1}\right|\right) \sigma_{2} \\
& \left.+\left(x+t_{1}-\left|x-t_{1}\right|\right) \sigma_{1}\right]
\end{aligned}
$$

para $0 \leq 2<t_{1} \circ t_{1}+d<x<t_{2} \circ t_{2}+d<x$. Lis Ec (3.15) implica onie $g_{n j}(. t)=0 y$ $h_{a j}(x)=-1$ para $|x| \in\left\{\left(t_{1}-a / 2 . t_{1}+d+a / 2\right)\right\} \cup\left\{\left(t_{2}-a, 2 t_{2}+d+a / 2\right)\right\}$.

El sistema es simétricc y sé cumple

$$
a_{n i}(y)=g_{0 i}(-y)
$$


entonces en la ecuación 3.11, usando esta propiedad podemos cambiar los límites de integración (ver apéndice A), cambiando $-y$ por $y$ en la región donde y es negativo por $y$, además $\mathrm{zdz}=\mathrm{sds}$ e integrando en coordenadas cilíndricas, tenemos

$$
g_{\alpha i}(x)=\exp \left\{-\beta u_{\alpha i}(x)+2 \sum_{m=1}^{2} \rho_{m} \int_{y=0}^{\infty} \int_{z=0}^{\infty} \int_{\phi=0}^{2 \pi} c_{i m}(s) h_{\alpha i}(y) z d \phi d z d y\right\}
$$

tal que

$$
\begin{aligned}
g_{\alpha i}(x) & =\exp \left\{-\beta u_{\alpha i}(x)+2 \pi \int_{t_{1}+d_{1}+t_{2}+d_{2}+a / 2}^{\infty} K(x, y) \rho_{\alpha s}(y) d y\right. \\
& -2 \pi \rho_{T} \int_{t_{1}+d_{1}+t_{2}-a / 2}^{t_{1}+d_{1}+t_{2}+d_{2}+a / 2} K(x, y) d y+2 \pi \int_{t_{1}+d_{1}+a / 2}^{t_{1}+d_{1}+t_{2}-a / 2} K(x, y) \rho_{\alpha s}(y) d y \\
& -2 \pi \int_{t_{1}-a / 2}^{t_{1}+d_{1}+u / 2} K(x, y) d y+2 \pi \int_{0}^{t_{1}-a / 2} K(x, y) \rho_{\alpha s}(y) d y \\
& +2 \pi z_{i} \int_{0}^{t_{1}-a / 2} L(x, y) \rho_{\alpha d}(y) d y+2 \pi z_{i} \int_{t_{1}+d_{1}+a / 2}^{t_{1}+d_{1}+t_{2}-a / 2} L(x, y) \rho_{a d}(y) d y \\
& +2 \pi z_{i} \int_{t_{1}+d_{1}+t_{2}+d_{2}+a / 2}^{\infty} L(x, y) \rho_{\alpha d}(y) d y \\
& +\frac{2 \pi \beta e^{2} z_{i}}{\varepsilon} \int_{t_{1}+d_{1}+t_{2}+d_{2}+a / 2}^{\infty}(x+y+|x-y|) \rho_{\alpha d}(y) d y \\
& +\frac{2 \pi \beta e^{2} z_{i}}{\varepsilon} \int_{t_{1}+d_{1}+a / 2}^{t_{1}+d_{1}+t_{2}-a / 2}(x+y+|x-y|) \rho_{\alpha d}(y) d y \\
& +\frac{2 \pi \beta e^{2} z_{i}}{\varepsilon} \int_{0}^{t_{1}-a / 2}(x+y+|x-y|) \rho_{\alpha d}(y) d y
\end{aligned}
$$

Donde se ha definido

$$
K(x, y) \equiv \int_{x+y}^{\infty} s c_{s}(s) d s+\int_{|x-y|}^{\infty} s c_{s}(s) d s
$$

y

$$
L(x, y) \equiv \int_{x+y}^{\infty} s c_{d}^{s r}(s) d s+\int_{|x-y|}^{\infty} s c_{d}^{s r}(s) d s
$$

Debe de recordarse que $c_{s}(s)$ y $c_{d}^{s r}(s)$ son iguales a cero para $s>0$, ver apéndice $\mathbf{B}$.

\subsection{Carga Inducida en el Fluido}

Una placa cargada en un fluido cargado induce una inhomogeneidad en el fluido debida a dos factores: su volumen excluido y el campo eléctrico generado por esta misma. Para alcanzar la condición de equilibrio termodinámico, en el fluido debe inducirse una carga tal que cancele la carga de la placa cargada. Para el sistema de cuatro placas inmersa en un electrolito, la condición de electroneutralidad se debe satisfacer de la misma forma. Es decir, 
la carga neta inducida cancela la carga total sobre tocias las supeticies de las parerles de las placas, i.e.,

$$
2 \sigma_{2}+2 \sigma_{T}^{\prime}=0
$$

Donde hemos definido $2 \sigma_{T}$ como la carga tcial sobre las placas (recuerde qut por simetría la carga sobre las placas del lado derecho es igual a la de las placas del lado izquierdo) y $2 \sigma_{T}^{\prime}$ como la carga total inducida en el fluido, i.e.,

$$
2 \sigma_{T}=2 \sigma_{1}+2 \sigma_{2}+2 \sigma_{3}+2 \sigma_{4}
$$

donde $\sigma_{1} \cdot \sigma_{2} \cdot \sigma_{3}$ y $\sigma_{4}$ están representados en la $\mathrm{Fig.} \mathrm{3.2.} \mathrm{y}$

$$
2 \sigma_{T}^{\prime}=2 \sigma_{1}^{\prime}+2 \sigma_{2}^{\prime}+2 \sigma_{3}^{\prime}+2 \sigma_{4}^{\prime}
$$

donde

$$
\begin{aligned}
& \sigma_{1}^{\prime}=e \int_{0}^{t_{1}-a_{i}{ }^{\prime}} \rho_{a n}(y) d y \\
& \sigma_{2}^{\prime}=e \int_{t_{1}+d_{1}+a / 2}^{t_{1}+d_{1}+t_{2}-a_{1} / 2} \rho_{c-d}(y) d y \\
& \sigma_{3}^{\prime}=e \int_{t_{1}+d_{1}+t_{2}-a_{2} / 2}^{t_{1}+d_{1}+a / 2} p_{\alpha d}(y) d y \\
& \sigma_{4}^{\prime}=e \int_{t_{1}+d_{1}+t_{2}+d_{2}+a / 2}^{\infty} \rho_{a d}(y) d y
\end{aligned}
$$

En general se tiene que

$$
\sigma_{1} \neq \sigma_{1}^{\prime}, \ldots, c_{4} \neq c_{4}^{\prime}
$$

obsérvese que para los cálculos se toman eu cuentu todas las cargas de las placas. La carga total de las placas un lado es

$$
\sigma_{T}=\sigma_{1}+\sigma_{2}+\varepsilon_{3}+\sigma_{4}
$$

La carga total inducida de un lado es

$$
\sigma_{T}^{\prime}=\sigma_{1}^{\prime}+\sigma_{2}^{\prime}+\sigma_{3}^{\prime}+\sigma_{4}^{\prime}
$$

la carga inducida del lado derecho del origen es (ver fig.3.2)

$$
\left.\sigma_{1}^{\prime}+\sigma_{2}^{\prime}+\sigma_{3}^{\prime}+\sigma_{4}^{\prime}=-e \int_{0}^{l_{8}} g_{a u l}(y) r_{y} y-\epsilon \int_{l_{5}}^{l_{2}} s_{i x}(y) d y-\cdots \int_{l_{1}}^{c o} g_{a x i}^{\prime} y\right) d y
$$

y la carga inàiscida del lado izquierdo del crigen es (ver fig. 3.2)

$$
\sigma_{1}^{\prime}+\sigma_{2}^{\prime}+\sigma_{3}^{\prime}+\sigma_{4}^{\prime}=-e \int_{0}^{-l_{s}} g_{a d}(y) d y-e \int_{-y_{3}}^{-l_{4}} g_{x d}(y) d y-\epsilon \int_{-l_{3}}^{-\infty} g_{w y}(y) d y
$$




$$
\begin{gathered}
l_{8}=t_{1}-a / 2 \\
l_{5}=t_{1}+d_{1}+a / 2 \\
l_{4}=t_{1}+d_{1}+t_{2}-a / 2 \\
l_{1}=t_{1}+d_{1}+t_{2}+d_{2}+a / 2
\end{gathered}
$$

\subsection{La presión neta sobre las placas.}

La fuerza sobre las placas inmersas en una solución electrolítica es uno de los parámetros más relevantes a ser considerado en el presente estudio. Como ya ha sido mostrado en trabajos anteriores donde se estudia la interacción efectiva entre dos placas $[22,79,80]$, la presión sobre una de las placas puede ser obtenida a partir de los perfiles de concentración iónica. De estos estudios previos sabemos que el cálculo de la presión sobre las placas se obtiene como la diferencia de presiones sobre las paredes de ambos lados de la placa, y esta diferencia se hace para cada placa. De manera general, la presión sobre una placa puede expresarse como la suma de dos contribuciones,

$$
P=P_{C}+P_{E}
$$

donde

$$
P_{C}=\left(\rho_{L}-\rho_{R}\right) k_{B} T
$$

es la contribución de contacto, siendo $\rho_{L}$ la concentración total de partículas en contacto con la parte izquierda de la placa y $\rho_{R}$ la concentración total de partículas en contacto con la parte derecha de la placa. $P_{E}$ es la contribución electrostática que actúa sobre una placa, cuyas contribuciones son debidas a las cargas superficiales en las paredes de las otras placas y las cargas inducidas en el fluido. En general, para una pared cargada cuya carga total sobre su superficie de área $A$ es $Q$, sabemos que la fuerza eléctrica sobre la pared es $F_{E}=Q E$, donde $E=\frac{\sigma_{E}}{2 \varepsilon}$ es el campo eléctrico producido por la densidad de carga por unidad de área, $\sigma_{E}$, incluye la densidad de carga superficial de otra u otras placas paralelas más las densidades de carga inducidas en el fluido. Es importante señalar que esta expresión es válida siempre que la inhomogeneidad en el fluido sea producida por geometría de placas paralelas. Por lo tanto, la contribución electrostática de la presión sobre la pared es

$$
P_{E}=\frac{F_{E}}{A}=\left(\frac{Q}{A}\right)\left(\frac{\sigma_{E}}{2 \ell}\right)
$$

Debemos señalar que para el cálculo de la presión se toman en cuenta todas las cargas de las placas y las cargas inducidas en el fluido, pues todas influyen para la presión neta sobre una 
placa. Para efectos de cálculo consideremos las fuerzás hacia la izquierda como negativas y las fuerzas hacia la derecha como positivas.

De las expreciones (3.32) y (3.33) se desprende que, pära una las placas interiores la presión neta está dada por

$$
P_{1}=k_{B} T\left(\rho_{L}^{(1)}-\rho_{R}^{(1)}\right)+\left(\frac{\sigma_{1}+\sigma_{2}}{2 \varepsilon}\right)\left(2 \sigma_{1}^{\prime}+\sigma_{1}+\sigma_{2}\right)
$$

y para las placas externas

$$
P_{2}=k_{B} T\left(\rho_{L}^{(1)}-\rho_{R}^{(1)}\right)+\left(\frac{\sigma_{3}+\sigma_{4}}{2 \Xi}\right)\left(2 \sigma_{1}^{\prime}+2 \sigma_{2}^{\prime}+2 \sigma_{3}^{\prime}+2 \sigma_{1} \div 2 \sigma_{2}+\sigma_{3}+\sigma_{4}\right)
$$

donde, $\sigma_{1}^{\prime}, \sigma_{2}^{\prime}, \sigma_{3}^{\prime}$ son las cargas induciáas en las diferentes regiones del espacio y donde se ha definido

$$
\begin{aligned}
& \rho_{L}^{(i)}=\sum_{m=1}^{2} \rho_{m} g_{a m}\left(t_{1}-\frac{a}{2}\right) \\
& \rho_{R}^{(1)}=\sum_{m=1}^{2} \rho_{m} g_{a m}\left(t_{1}+d_{1}+\frac{a}{2}\right) \\
& \rho_{L}^{(2)}=\sum_{m=1}^{2} \rho_{m} g_{a m}\left(t_{1}+d_{1}+t_{2}-\frac{a}{2}\right) \\
& \rho_{R}^{(2)}=\sum_{m=1}^{2} \rho_{m} g_{a m}\left(t_{1}+l_{1}+l_{2}+d_{z}-\frac{a}{2}\right)
\end{aligned}
$$

\subsection{Análisis de Resultados}

En esta parte comentaremos los resultados para el mocielo que hemos desarrollado. Partiendo del arreglo más simple, consideramos las condiciones para que en el sistena se conserve la simetría espacial con respecto al plano de simetría (en $x=0$. ver fig. 3.2 ), además el programa está realizado sólo para condiciones de sirietríc. Hemos aplicado en todas las paredes de las placas una densidad de carga superficial $\tau$ igual y uniforme. El aricho de las placas $d_{1} d_{2}$, es igual a $d=a / 2$. La simetría antes mencicnarda corresponde a que las distancias del plano de simetría a las placas de la izquierda scan iguaies a la distancias del mismo plano a las placas de la derecha. Entonces sólo analizamos al iado c̉erecto de este plano, pues, las gráficas son simétricas. A las dos placas de adentro las lamamos placas interiores (o placas 1) y a las placas de afuera las llamamos placas exteriores (o placas 2), ver fig.3.2

Por medio de las ecunciones obienidas cn la teoria. se hace un programa para la computadora y se obtienen los resultados muméricos. El diámetro de los iones es $u$, ron dimensiones de $4.25 \AA$, ver fig. 3.2. la separación entre las placas internas (plecos 1 ) es $2 t_{1}$. que es la región 1, pero sólo representamos la distancia $t_{1}$ en las gráfi: as. ver tig. 3.2 , cue es lía distancia del centro del sistelia en el eje de simetría hast a la rlaca? de la derecha en 'á parte de la pared izquierda de esta misma placa; $t_{2}$ es la separación entre la placa interna y la placa externa desde la pared derecha de la placa interna a la partd izquierda de la pared externa, que es la región II. observe la fig. 3.2. En todos los cólculos tenovos ma tunperatura de 'T=298K, la 
constante dieléctrica del fluido es $\epsilon=78.5$. La constante dieléctrica de las placas fue elegida igual que la constante dieléctrica del solvente.

Los perfiles de concentración reducida (PCR), la presión neta $P_{1}$ y $P_{2}$ sobre las respectivas placas, así como la carga inducida $\left(\sigma_{i}\right)$ en todas las regiones son calculados, para electrolitos 1:1 y 2:2 con varias concentraciones de bulto y densidades de carga superficial sobre las paredes de las placas.

Los perfiles de concentración iónico reducido, corresponden a la concentración dividida entre la concentración de bulto. Los cálculos fueron realizados variando las separaciones entre las placas, pero siempre manteniendo simetría con respecto al centro del sistema en el plano de simetría. En los cálculos ha sido considerada la distancia de máximo acercamiento de los iones a las placas de $a / 2$ (pues la referencia está en los centros de los iones). Hemos considerado arbitrariamente la carga sobre las placas positiva, si la carga es negativa el problema es equivalente. En todas las gráficas de los PCR, las curvas de los contraiones están representadas por encima de las curvas de los coiones, y las diferencias importantes se observan en los PCR de los contraiones. Las diferencias en los PCR de los coiones son menores y no se observan claramente en las gráficas.

En la figura 3.3 presentamos los PCR para un fluido cargado 1:1, con una concentración de $0.1 \mathrm{M}$, y con una carga sobre cada pared de $0.1 \mathrm{C} / \mathrm{m}^{2}$. Cada figura contiene tres gráficas, para tres distancias $D=t_{1}+d+t_{2}$, diferentes, y con la condición de que la distancia $t_{1}$ es fija en cada gráfica, ver fig.3.2. Se pueden observar los PCR en cada región de los iones positivos y negativos (coiones y contraiones respectivamente), que se encuentran cerca de las paredes de las placas, y se marcan las 3 regiones que delimitan las placas. El ancho de las placas no es representado en las gráficas, para mejor visibilidad de las curvas. Los PCR de los sistemas de las dos placas simples (2P) y de cuatro placas (4P) se representan en la misma gráfica, y son los perfiles obtenidos en las diferentes regiones entre las placas y fuera de éstas.

Los resultados correspondientes al modelo de $2 \mathrm{P}$ se representan junto con los resultados de las placas internas de las $4 \mathrm{P}$, superponiendo las $2 \mathrm{P}$ simples encima de las dos placas internas. Asímismo también se superponen las $2 \mathrm{P}$ simples con las placas interna y externa de las mismas $4 \mathrm{P}$, y se trazan las curvas de los perfiles de ambos sistemas con diferente tonalidad de color (lineas obscuras son los resultados de las 4P, y las lineas claras los resultados de las $2 \mathrm{P}$ ). Los perfiles en la región del exterior de las $2 \mathrm{P}$ sólo se representan en la región exterior de las 4P. En cada gráfica se trazan los PCR de los iones en las tres regiones que separan las placas, para el modelo de las $4 \mathrm{P}$, y las dos regiones superpuestas del modelo de las $2 \mathrm{P}$.

Los resultados son analizados, observando las diferencias de los perfiles en ambas geometrías. Puede observarse que, para el sistema de las 4P, al cerrar más las placas externas el confinamiento aumenta y las partículas entre las placas 1, cambian más sus propiedades con respecto a las $2 \mathrm{p}$. La carga de las placas 2 afecta más a las partículas en la región I. Si las placas 1 están muy lejos de las placas 2, la interacción entre las placas 1 y las placas 2 desaparece y los perfiles de concentración de las $4 \mathrm{P}$ se sobreponen exactamente a los perfiles de $2 \mathrm{P}$, pareciéndose al modelo de $2 \mathrm{P}$.

En esta misma fig 3.3, en las gráficas se mantiene el tamaño de la región I fija, con $\left.t_{1}=1.4 a / 2\right)$. En esta región vemos una gran diferencia entre la distribución de contraiones para ambas geometrías, siendo mayor en las 4P. Existe mayor concentración de partículas en las $4 \mathrm{P}$ que en las $2 \mathrm{P}$, es decir hay más confinamiento en las $4 \mathrm{P}$. En la región II, se pueden observar los mismos cálculos para las placa 1 y placa 2 a las distancias entre ellas 
de $t_{2}=3.6,4.6,6.6(a / 2)$, una distancia $t_{2}$ para cada gráfica. Obsévese que cerca de la placa interior se separan las curvas de los 2 sistemas (obscura y clara), y es mavor la separación para cuando hay mayor confinamiento ( $D$ más pequeño), la concentración para las 4 P siempre es mayor en la región I y la región 2. Las curvas se vuelven a juntar cerca de la placa 2 en las distribuciones de ambas geometrías, en todas las gráficas.

Por último, en la región III, en todas las gráficas de csta figura, practicamente las curvas de los dos sistemas coinciden (se enciman) y no hay diferercias significativas entre las $2 \mathrm{P}$ y las $4 \mathrm{P}$. Es importante recalcar que a medida que se aumenta la distancia ") (disminuye el confinamiento en algunas regiones), las diferencias en los resultados de las dos geometrías disminuyen. Observese también que cuando la separación $t_{2}$ aumenta, los contraiones en las $4 \mathrm{P}$ se acomodan más parecido a los contraiones de las $2 \mathrm{P}$ cerca de la placa exterior. Los iones en el modelo de las 4P que se encuentran en la región I, muestran una conducta de mayor confinamiento, que en las $2 \mathrm{P}$ por estar entre cuatro placas.

Las fuerzas producidas por las placas exteriores afectan a los ionas que se encuentran en la región I. En la figura 3.4 presentamos los perfiles de concentracićn entre las dos geometrías, para un electrolito $1: 1$, con una concentración de 0.11 Y una derisidad de carga de $0.1 \mathrm{C} / \mathrm{m}^{2}$ en cada pared, cuyas condiciones son similares a ia figura anternor. pero ahora cambia la distancia $t_{1}$. Las placas internas se encuentran más separadas cue eri la figura anterior, $t_{1}=2.9(a / 2)$ y $t_{2}=2.1,3.1,5.1(a / 2)$. En este casc podemos ver que ios PCR para los contraiones en la región II, presentan un fuerte confinamiento ́álrededor de $g_{-}=500$, no es represeniado en la gráfica, pues es muy grande), este confinamiento es mayor al que presentan los contraiones en la región I pero en la figura antericr, pies la separación entre la placa 1 y la placa 2, es menor que la separación entre lis placias 1 de la figura anterior, sin embargo las distribuciones presentan una conducta similar er ambas regiones. En el modelo de las 4P, los PCR de los contraiones siguen presentando las mavores diferencias cerca de las paredes de las placas interiores, aunque cerca de las paredes exteriores los PCR de los contraiones muestran una conducta similar en las 2 geometrias.

En la figura 3.5 presentamos los PCR producidos por las 23 y las 4P en un fluido de electrolito $2: 2$, con una concentración de $0.5 \mathrm{M}$ y una densidar de carga de $0.272 \mathrm{C} / \mathrm{m}^{2}$ en las paredes. Las tres gráficas, se refieren a las distancias de $D=6,7,9(a / 2)$. un valor para cada gráfica y para $t_{1}=1.4(a / 2)$ fijo en cada gráfica. Los perfiles presentan resultados característicos de un fluido divalente. Para este caso. los perfiles debidos a Ies $2 F \%$ las $4 \mathrm{P}$ presentan otras características diferentes al caso monovalente. Se observa una conducta os:ilatoria de los perfiles para cuando las placas están más separadas, cosa ciue va ha siao observada en estudios anteriores para fluidos divalentes. Cuando las placas est fon muy cercanas entre si, no alcanzan a oscilar las curvas.

Los valores de los perfiles de los contraiones entre ias dos ular as smiples aparecen por arriba de los perfiles de las cuatro las placas, en la región I, con la tistancia de $l)=6.0(a / 2)$, es decir son más grandes las concentraciones de las $2 \mathrm{P}$. la conducta se iurititió al caso monovalente. las gráficas muestran que en la región 1. ẹ las 2P. hay más contraiones, que en las 4p. Se observa que las placas 2 afectan de otra manera a las piacas 1: coii esta roncentración, el confinamiento fue reducido un poco. Sin embargo pera la misrat $\ell_{1}$ : $\left.: D=-i n / 2\right)$ y $9(a / 2)$ vuelven a ser ligeramente menores los PCF de las $2 \mathrm{P}$ que los verfiles de la geometria de las 4P. tsta observación no se puede distinguir en el dibrio. En ia region Il los varbiles son muy diferentes cerca de la pared 1 y la 2 y éstas a la vtz al caso monnoiente. Cerca do la pared 1 
en todas las gráficas los perfiles de las $4 \mathrm{P}$ están por debajo de los perfiles de las $2 \mathrm{P}$ simples, $\mathrm{y}$ sólo son mayores cerca de la pared 2 cuando $D=6(a / 2)$ y $7(a / 2)$, aunque en $D=7(a / 2)$ no se distingue claramente en la gráfica. Los contraiones se acomodan de otra manera diferente para $D=9(a / 2)$. Podemos observar que el perfil de las $2 \mathrm{P}$ simples para los contraiones se vuelve otra vez ligeramente mayor cerca de la pared externa, no se distingue claramente en la gráfica. El acomodo de las partículas se debe a la entropía en el sistema, estas partículas al estar más concentradas y con mayor carga buscan espacios donde las fuerzas entre ellas lo permiten. También podemos observar que las partículas entran y salen según la separación de las placas.

En la figura 3.6 presentamos los PCR para un fluido con condiciones similares a la gráfica 3.3. En esta figura se observan tres gráficas de los PCR de coiones y contraiones, para el modelo de las $4 \mathrm{P}$ con $t_{1}=2.9(a / 2)$ fijo, y superponiendo las $2 \mathrm{P}$ encima de las placas internas $\mathrm{y}$ de las placas interna y externa de las $4 \mathrm{P}$ separadamente. Las tres gráficas corresponden cada una a $D=6(a / 2), 7(a / 2)$ y $9(a / 2)$ respectivamente. Observese que para $D=6(a / 2)$ y $7(a / 2)$, en la región $\mathbf{I}$, es menor la concentración de contraiones en las $4 \mathbf{P}$ que en las $2 \mathrm{P}$, sin embargo para $D=9(a / 2)$ los PCR se vuelven a invertir en esta región, aunque no se distingue en la figura, la concentración de contraiones es mayor en las $4 \mathrm{P}$. Para $D=6(a / 2)$ y $7(a / 2)$ en la región $\mathrm{I}$, se ha disminuido el confinamiento de las partículas en las $4 \mathrm{P}$, sin embargo para $D=9(a / 2)$, aumentó.

En la región II se observa un aumento en la concentración de contraiones de una manera importante (no se presenta en la gráfica, pues es alrededor de $g_{-}(x)=320$ ), pues se ha reducido mucho el espacio entre las partículas, pero sigue siendo mayor la $g_{-}(x)$ para las 4P. Parte del confinamiento de la región I pasa a la región II. Si comparamos con la gráfica correspondiente a la distancia $D=6(a / 2)$ y $t_{1}=1.4(a / 2)$, en la figura anterior, vemos que el confinamiento es mayor porque los iones en la región II de esta última gráfica, se encuentran a una separación de pared a pared de $2.1(a / 2)$, y el perfil de contraiones de las 4P aparece por arriba del perfil de las $2 \mathrm{P}$, este comportamiento si es observado en el caso monovalente para las mismas distancias.

En la región I los perfiles de $2 \mathrm{P}$ aparecen por arriba del perfil de las $4 \mathrm{P}$ cerca de las paredes internas, sin embargo a medida que nos alejamos de la pared los perfiles se invierten, y las concentraciones para las $4 \mathrm{P}$ son mayores que los perfiles de $2 \mathrm{p}$, (no se alcanza a distinguir en las gráficas), para las distancias $D=6(a / 2)$ y $7(a / 2)$. Otra observación importante en la región I, es que los perfiles de contraiones y coiones para las $2 \mathrm{P}$ se cruzan lejos de la pared, para las $D=6,7,9(a / 2)$, cosa que sólo sucede en las $4 \mathrm{P}$ para $D=9(a / 2)$ y también para esta distancia $D$, el perfil de contraiones de $2 \mathrm{P}$ está ligeramente por arriba del perfil de las $4 \mathrm{P}$ lejos de la pared 1, pues cerca de la pared las $g_{-}(x)$ son mayores para las 4P. Para esta misma $D$, los perfiles de las dos especies se parecen mucho en las dos geometrías.

En la región 11 para $t_{1}=2.9(a / 2)$ y $D=7(a / 2)$ y $9(a / 2)$ se observan menos diferencias en los perfiles para las dos geometrías, comparados con las gráficas para $t_{1}=1.4(a / 2)$ y $D$ correspondientes, con un electrolito divalente, pero sigue habiendo diferencias cerca de las placas interiores en menor proporción. Las gráficas en los casos divalentes para las dos placas y las cuatro placas presentan importantes diferencias con respecto al caso monovalente. Se nota una conducta oscilatoria de las curvas principalmente. Se observa que al estar más separadas las placas interiores $(D=\tau(a / 2)$ y $9(a / 2))$ se afecta menos a la región II, y los PCR de las dos geometrías se ven muy similares. Podemos decir que si aumentamos el número 
de placas, mientras se encuentren muy separadas, no se afectan entre ellas, podemos esperar un comportamiento similar al modelo de una placa o de dos placas.

En el apéndice presentamos cuatro gráficas, 2 de ellas correspondientes a las separaciones de $D=6,7,9,(a / 2)$ con $t_{1}=2.4(a / 2)$ monovalente y divalente, y las otras con $D=7,9,13(a / 2)$ y $t_{1}=3.4(a / 2)$, con electrolitos monovalente y divalente también.

En las figuras 3.7 y 3.8 vemos las curvas de la presión $P_{1}$ contra distancia $t_{1}$, para varias distancias fijas del centro de las placas a la placa $2(D)$, es decir sólo varian las distancias $t_{1}$ y $t_{2}$, con valores de $D=6,9,13(a / 2)$ fijos, podemos observar los perfiles de presión o fuerza sobre area (adimensional y cuando la presión es igual a 1 corresponde a la presión de $\rho k_{B} T_{\text {: } \rho}$ es la concentración de bulto), y $t_{1}$ en unidades de ( $\left.a / 2\right)$, las placas internas se mueven hacia la derecha o izquierda, manteniendo las placas exteriores fijas a una distancia $D$. Obsérvese también que aparece la presión sobre las placas para la geometría de $2 \mathrm{P}$, superponiendo las dos placas simples con las placas 1 de la geometría de las 4P.

En la figura 3.7 las condiciones del fluido del sistema son un electrolito $1: 1,0.1 \mathrm{M}$ y carga sobre las paredes de $0.1 \mathrm{C} / \mathrm{m}^{2}$. Si se juntan las placas interiores aumenta la fuerza entre ellas y es repulsiva (positiva) y si aumenta la distancia entre ellas disminuye la fuerza entre las placas 1, pasando por cero y se vuelve atractiva (negativa), pues ahora las placas externas las empujan y las placas 1 sienten que se quieren juntar. Es importante observar el carácter monótono decreciente en todas las curvas a medida que aumenta la distancia $t_{1}$. También a medida que aumenta $D$ la pendiente de las curvas se hace más suave.

El valor de la fuerza para todas las curvas tiende a un valor constante cuando $t_{1}$ disminuye. Cuando las placas internas se juntan, $\left(t_{1} \rightarrow a / 2\right)$, y la repulsión es máxima, sin embargo las placas externas las empujan, aún a la distancia de máxirna separación entre la placa interior $\mathrm{y}$ exterior, disminuyendo la repulsión de las placas internas. El valor de la fuerza cuando $t_{1} \rightarrow a / 2$ para cada separación $D$ es parecido para todas las curvas (no se distingue en la gráfica). Sin embargo, no coinciden exactamente, pues la fuerza se ve afectada por la presencia de las placas externas. Por otra parte, podemos observar que para cuando el valor de $t_{1}$ es el más grande, para cualquier valcr de $D$, las fuerzas tienden a un valor muy parecido, cuyo valor de la fuerza es muy cercaro al valor para cuando $t_{1} \rightarrow a / 2$.

Cuando las placas internas se alejan, se van encontrando a las placas externas fijas; éstas últimas las empujan sintiendo a la vez las internas una atracción resultante a pesar de tener la misma carga. En el máximo de $t_{1}$ la fuerza sobre las placas internas es atractiva, si $t_{1}$ es mínimo las placas internas son influidas por las externas y disminuve su repulsión.

Es importante aclarar que sólo se alcarizan los valores de las fuerzas parecidas cuando las placas están muy próximas es decir cuando $t_{1}$ y $t_{2}$ tienen valores mínimos. Si no se alcanzan estas distancias las magnitudes de las fuerzas no se asemejan. Es claro, que las placas tanto internas como externas no se van a comportar como si estuvieran aisladas, pues las correlaciones de corto alcance se vuelven importantes a mavor confiramiento.

Para un fluido monovalente no oscilan las curvas, ésto se debe a la concentración, la valencia y las correlaciones de corto alcance. Al haber mencs partículas hey menos irteracción entre ellas y la fuerza eléctrostática prevalece y existe nenos aparitallamierto oiitrc las placas.

La presión para el sistema de $2 \mathrm{P}$ está casi encimario con la presión del caso en que $D=13(a / 2), y$ tiende a cero pues a medida de que se alejan las placas la interacción disminuye. Se observa que, entre más separadas estén las placas internas, más parecidas serán las presiones con las $2 \mathrm{P}$ simples. La presión para las $2 \mathrm{P}$ está ligeramente por arriba 


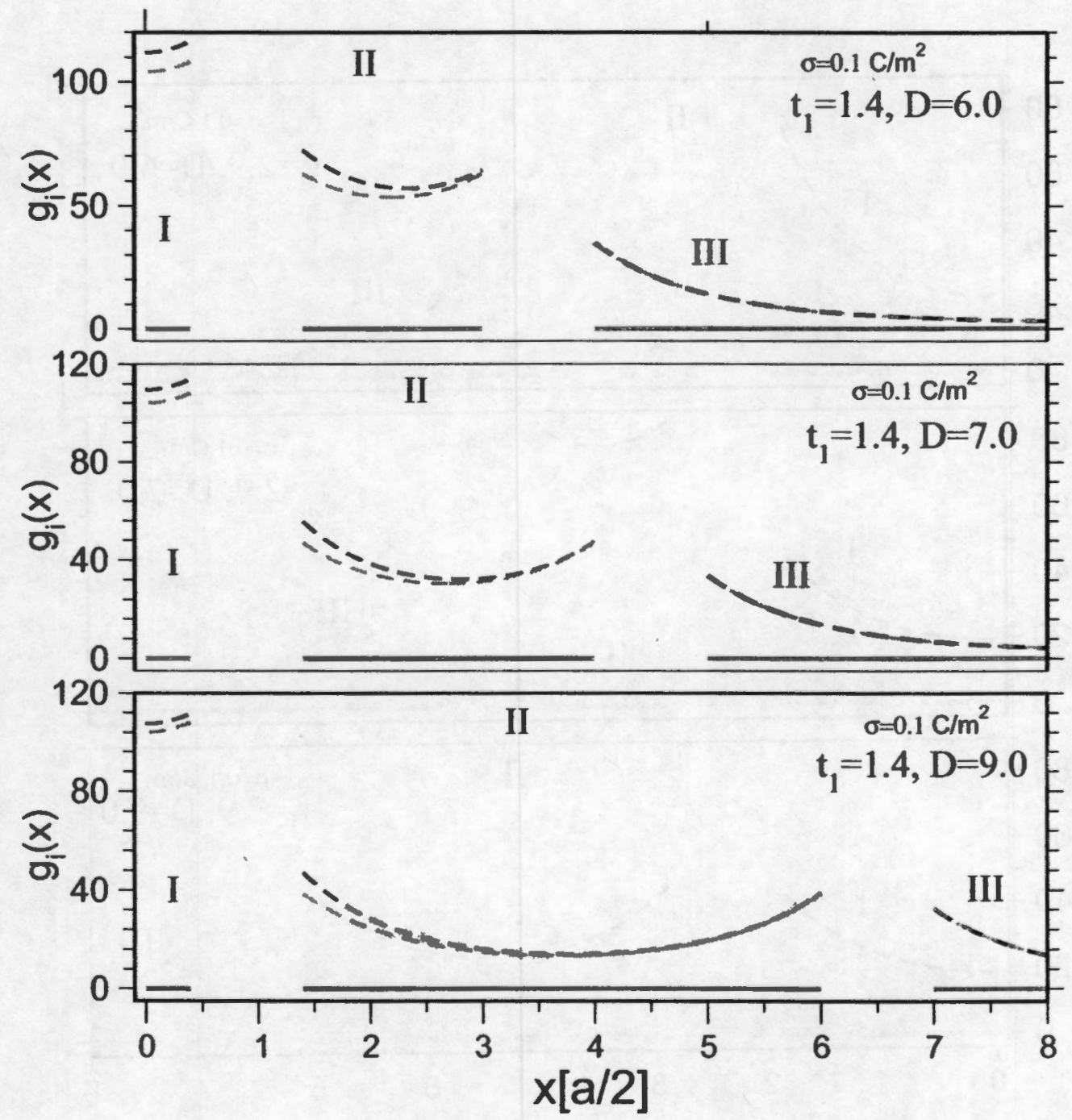

Figura 3.3: Perfiles de concentración de contraiones y coiones $\left(g_{-}(x)\right.$ y $g_{+}(x)$ respectivamente, $i=-,+$ ) como función de la distancia perpendicular a las placas $x$, para un electrolito 1:1 y $0.1 \mathrm{M}$, confinado entre cuatro placas cargadas con $\sigma_{1}=0.1 \mathrm{C} / \mathrm{m}^{2}=\sigma_{2}=\sigma_{3}=\sigma_{4}$, para las separaciones de placas $t_{1}$ y $D$ como se indican en la figura. La distancia $x$ es desde el centro del sistema, medido en unidades de $(a / 2)$. Las líneas punteadas y continuas se refieren a los contraiones y coiones respectivamente. Las lineas oscuras se refieren a las cuatro placas y las lineas claras a dos placas, el ancho de las placas no se representa en las gráficas. 

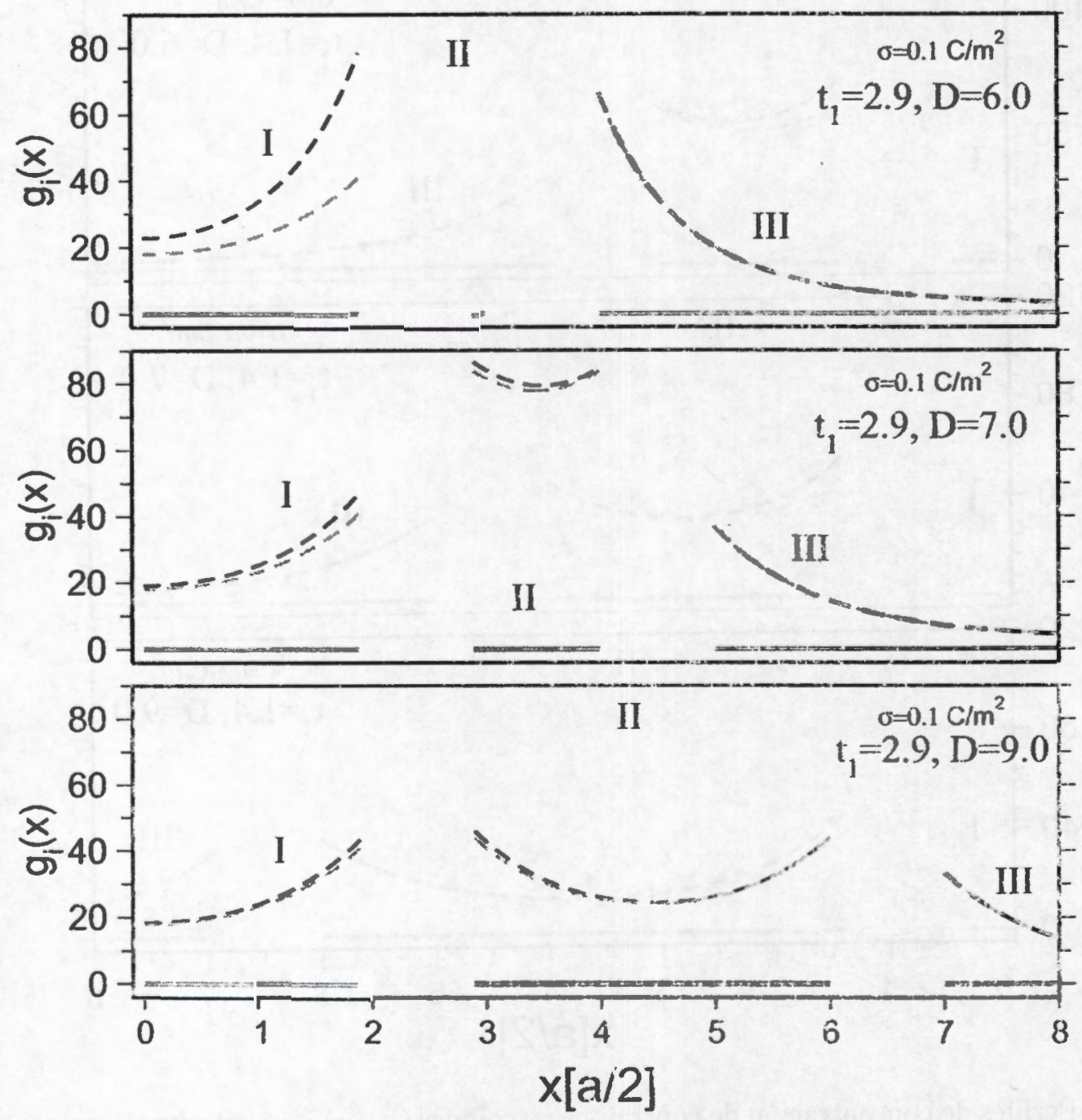

Figura 3.4: Perfiles de concentración de contraiones y coiones como función de la distancia perpendicular a las placas $x$, con las mismas características del fluido que en la figura anterior, para las separaciones de placas indicadas en la figura. Las lineas tienen la misma notación que en la figura 3.3 . 

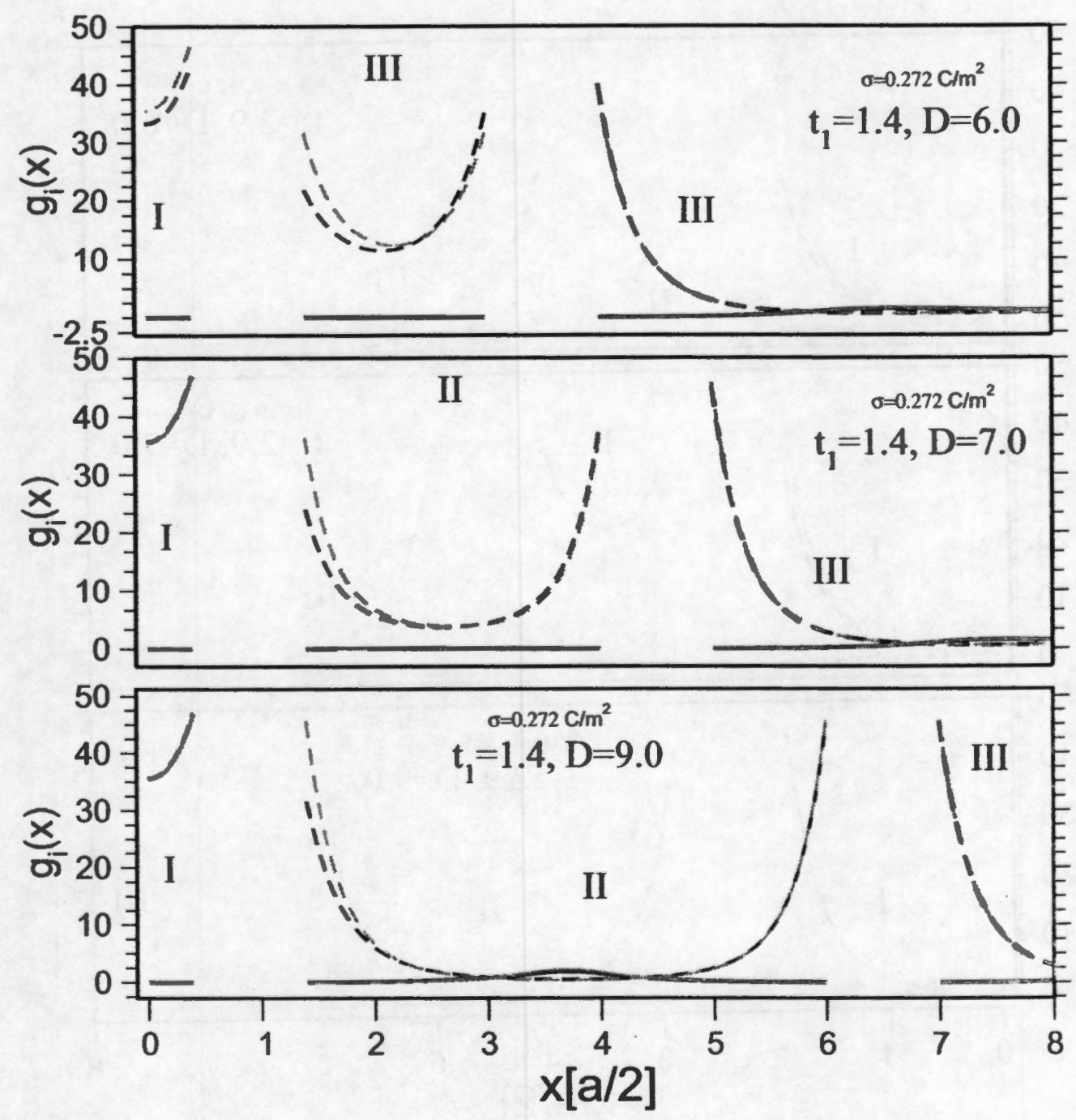

Figura 3.5: Perfiles de concentración de contraiones y coiones como función de la distancia perpendicular a las placas $x$, para un electrolito $2: 2$ y $0.5 \mathrm{M}$, confinado entre cuatro placas cargadas con carga superficial de $\sigma_{1}=0.272 \mathrm{C} / \mathrm{m}^{2}=\sigma_{2}=\sigma_{3}=\sigma_{4}$, para las separaciones de placas indicadas en la figura. Las lineas tienen la misma notación que en la figura 3.3. 

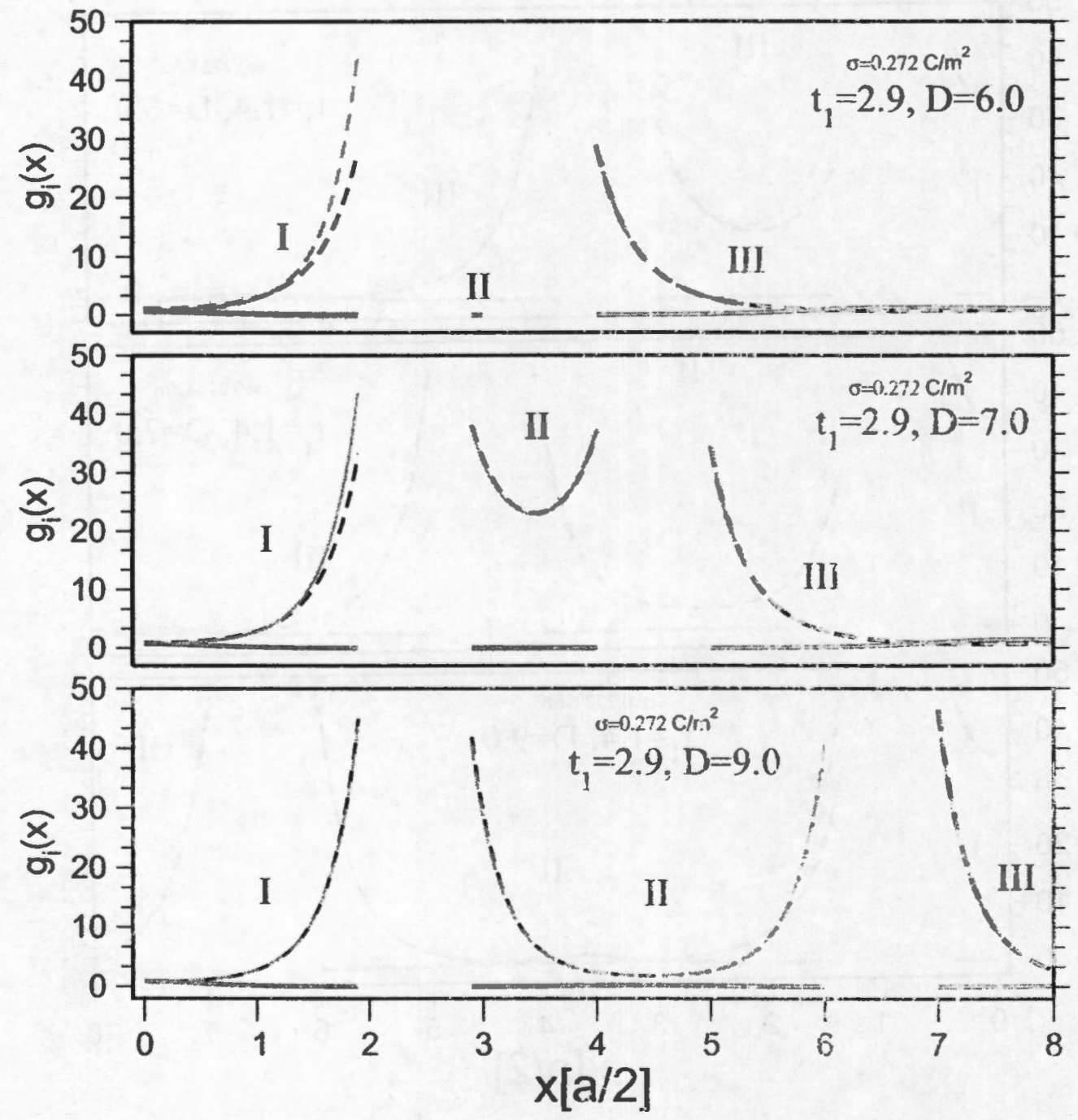

Figura 3.6: Perfiles de concentración de contraiones y coiones como función de la distancia $x$, para un electrolito $2: 2,0.5 \mathrm{M}$ y $\sigma_{1}=0.272 \mathrm{C} / \mathrm{m}^{2}=\sigma_{2}=\sigma_{3}=\sigma_{4}$ para las separaciones de placas indicadas en la figura. Las lineas tienen la misma notación que en la figura 3.3. 


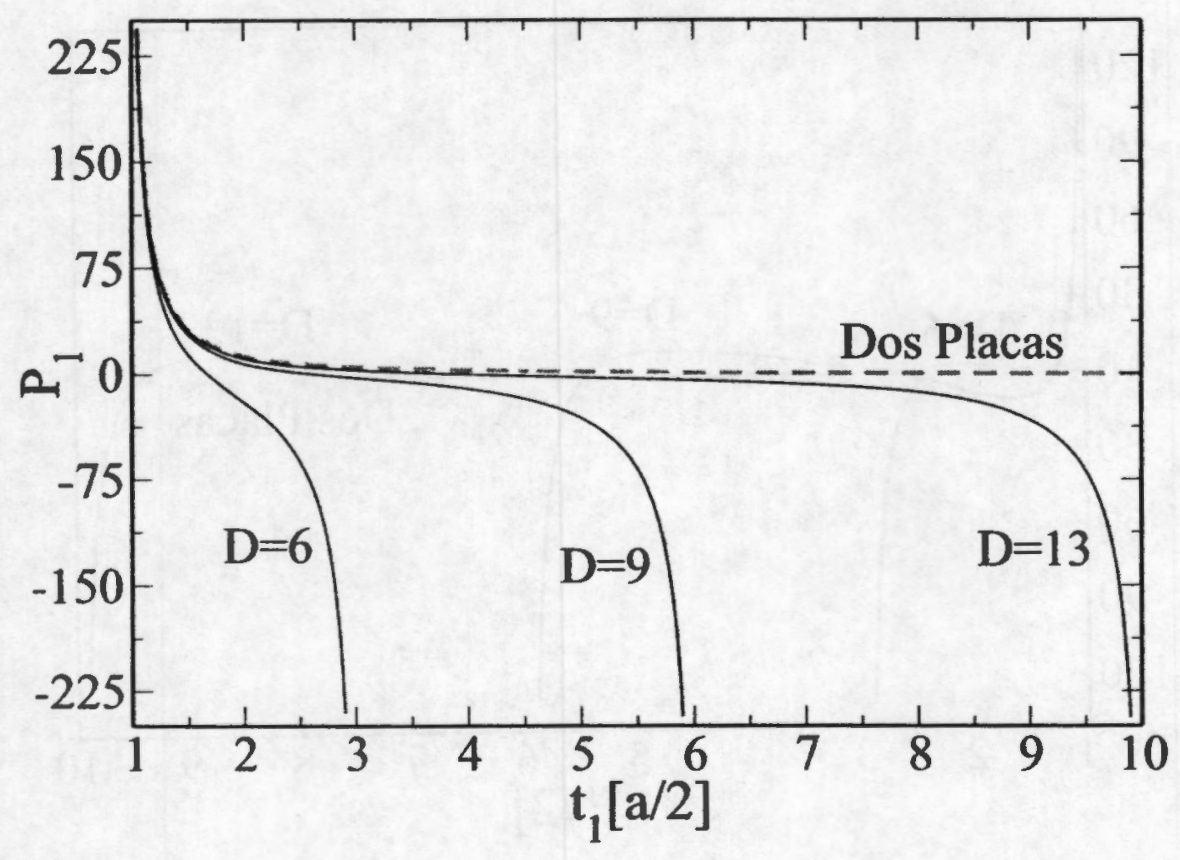

Figura 3.7: Gráfica que presenta los perfiles de presión neta sobre la placa 1, (4P) en función de la distancia entre el centro y la placa $1\left(t_{1}\right)$, las curvas corresponden a distancias diferentes del centro del sistema a la placa 2 , fijas, $D=6,9,13(a / 2)$, de izquierda a derecha. Para las condiciones $1: 1,0.1 \mathrm{M}, 0.1 \mathrm{C} / \mathrm{m}^{2}$. Aparece también la presión neta de cada placa en el sistema de $2 \mathrm{P}$, con linea clara y punteada, donde las dos placas e superponen con las placas 1 de las $4 \mathrm{P}$.

de las curvas para $D=13(a / 2)$ de las $4 \mathrm{P}$, sin embargo, es notoriamente diferente con los casos $D=9(a / 2)$ y aún más para $D=6(a / 2)$, no obstante, las cuatro curvas se asemejan en $t_{1} \rightarrow a / 2$.

En la figura 3.8, presentamos las curvas de $P_{1}$ sobre la placa 1, como función de $t_{1}$, para un electrolito $2: 2,0.5 \mathrm{M}$ y carga $0.272 \mathrm{C} / \mathrm{m}^{2}$ en las placas. En estas curvas, podemos observar como la fuerza tiene una conducta oscilante típica de un electrolito divalente. Las fuerzas para las placas 1 muy juntas o muy separadas en el caso divalente son de mayor magnitud que las fuerzas en el caso monovalente para las mismas separaciones de placas pues, como la presión es adimensional y está dividida entre $\rho k_{B} T$. Entonces, este factor es cinco veces mayor para el caso divalente rho vale $0.5 \mathrm{M}$, y el caso monovalente tiene concentración $0.1 \mathrm{M}$, aunque para el caso divalente tienen menor alcance. Además, en el caso divalente tienen mayor valencia los iones y al estar más concentrados, hay un mayor apantallamiento entre las placas que para el caso monovalente. La presión para el caso divalente no se mantiene monótona decreciente cuando la distancia $t_{1}$ crece. Existe un punto $\underline{t}_{1}$ de valor pequeño (1.1 $(a / 2)$ ) donde casi todas las curvas de $P_{1}$ (excepto para $D=6(a / 2)$ ) se vuelven atractivas, vuelven a ser repulsivas y de nuevo se hacen atractivas cuando $t_{1}$ tiende a su valor máximo. Para la distancia $D=13(a / 2)$, con valores de $t_{1}$ entre 4 y $6(a / 2)$, la presión pasa por otro mínimo donde la presión es negtiva. Así como para el caso monovalente, cuando $t_{1} \rightarrow a / 2 \mathrm{o}$ 


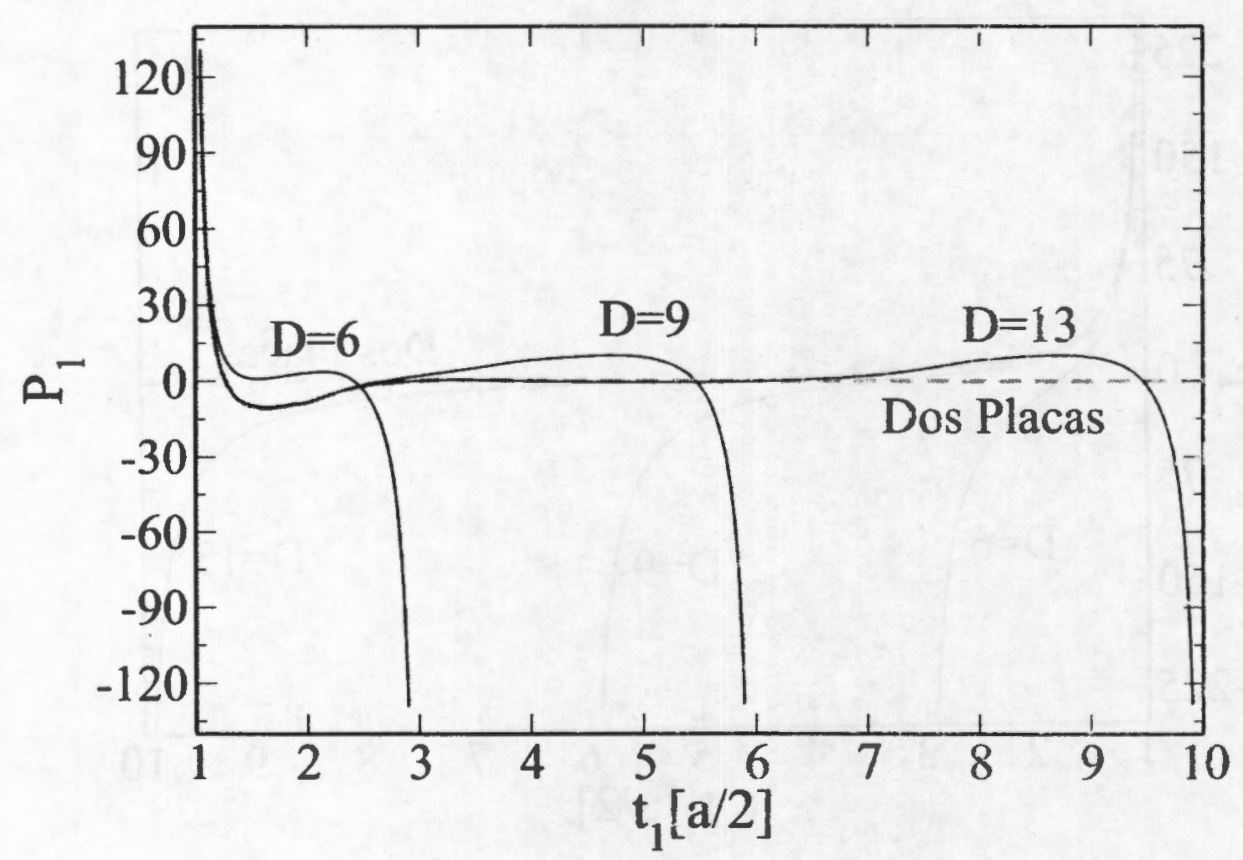

Figura 3.8: Gráfica que presenta los perfiles de presión neta sobre la placa 1, en función de la distancia entre el centro y la placa $1\left(t_{1}\right)$, las curvas corresponden a distancias diferentes del centro del sistema a la placa 2 , fijas, $D=6,9,13(a / 2)$, de izquierda a derecha. Para 2:2, $.5 \mathrm{M}, 0.272 \mathrm{C} / \mathrm{m}^{2}$. Aparece también la presión neta de cada placa en el sistema de $2 \mathrm{P}$, con linea clara y punteada, superpuestas con las placas 1 del sistema de $4 \mathrm{P}$.

cuando $t_{2} \rightarrow a / 2$ los valores de $P_{1}$ son muy cercanos, es decir, para electrolitos divalentes la fuerza cuando $t_{1}$ tiene valor máximo es igual al valor absoluto de la fuerza para cuando $t_{1}$ tiende al valor mínimo (a/2).

La presión para el sistema de $2 \mathrm{P}$, está practicamente encima de la curra de la presión, en la parte cercana a la placa 1 del caso en que $D=13(a / 2)$, y tiende a cero a medida de que se alejan las placas. Para el caso de las $4 \mathrm{P}$, cuando las placas internas se alejan, se encuentran a las placas externas y la influencia de estas placas se manifiesta en la presión sobre la placas 1. es por eso que al alejarse las $2 \mathrm{P}, \mathrm{y}$ no encuentran otra placas, su presión tiende a cero y ya no coinciden con las $4 \mathrm{P}$.

Se puede ver como en el caso monovalente que entre mayor sea la ciistancia $D, y$ mayor la separación entre las placas internas para las 4P, mavor parecido tendráu las presiones con las $2 \mathrm{P}$ simples. Entonces para estas distancias $D$ ya casi no existe interacción con las placas externas, hasta cuando la separación de las placas internas con las placas externas empieza a disminuir $\mathrm{y}$ las placas externas comienzan a ejercer influencia sobre las placas internas. Cuando la concentración de electrolito es mayor, el apantallamiento entre las placas es mayor. La existencia de las placas externas no disminuye notoriamente la repulsión de las placas internas para $D=13(a / 2)$, mientras no se acerque lo suficiente la placas 1 a la placas 2 . Se puede observar que para $D=6(a / 2)$, la presión cambia raáicalmente cor las $2 \mathrm{P}$, es decir a mayor confinamiento en las regiones 1 y 2, las fuerzas se modifican considerablemente. 


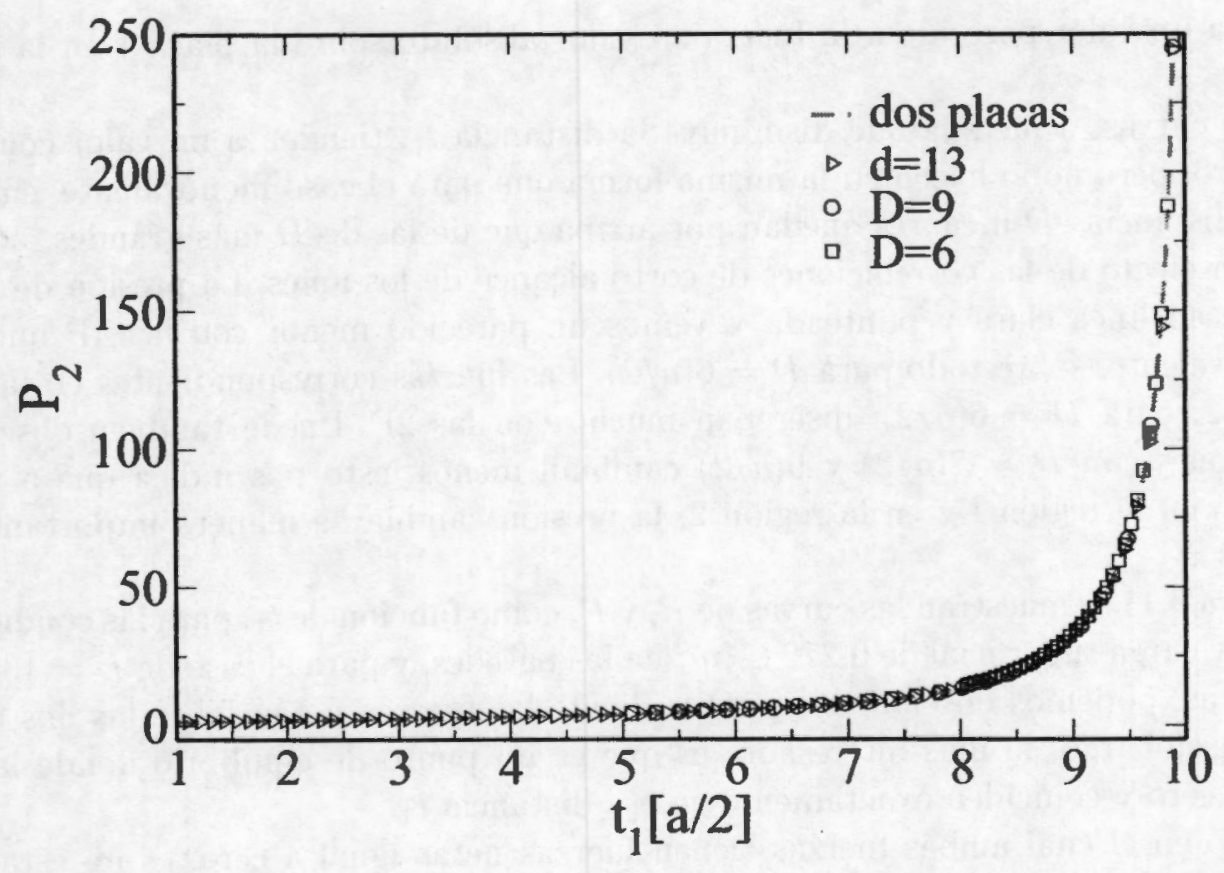

Figura 3.9: Gráfica que presenta los perfiles de presión neta sobre la placa 2, en función de la distancia entre el centro del sistema y la placa $1\left(t_{1}\right)$, las curvas corresponden a distancias diferentes del centro del sistema a la placa 2 , fijas, $D=6,9,13(a / 2)$, de izquierda a derecha. Para $1: 1,0.1 \mathrm{M}, 0.1 \mathrm{C} / \mathrm{m}^{2}$, La presión neta de cada placa en el sistema de $2 \mathrm{P}$, se representa con linea clara y punteada. La notación igual a la figura 3.7.

En la figura 3.9, se muestran las curvas de $P_{2}$, sobre la placa 2, como función de $t_{1}$, para las condiciones de $1: 1,0.1 \mathrm{M}$ y carga $0.1 \mathrm{C} / \mathrm{m}^{2}$ sobre las placas. Se puede observar como la fuerza se mantiene monótonamente creciente y repulsiva todo el tiempo cuando la distancia $t_{1}$ crece. Para este caso las curvas de $2 \mathrm{P}$ y de $4 \mathrm{P}$, son muy parecidas entre sí, cuando la placa 1 se mueve, no se afectan notoriamente las fuerzas $P_{2}$.

Cuando la distancia $t_{1}$ es máxima la fuerza sobre la placa 2 coincide con la fuerza sobre la placa 1 , en la misma $t_{1}$. Todas las curvas de $P_{2}$, a medida que disminuye la distancia $t_{1}$ decrecen, y tienden a un valor cercano al cero, aunque ese valor cercano a cero es diferente para todas las curvas de $P_{2}$ porque las distancias a las placas externas en cada curva es diferente. Esto se puede entender por la razón de que las placas internas están más alejadas de las placas externas en $t_{1} \rightarrow a / 2, y$ entonces éstas últimas sienten la menor fuerza de interacción con las placas internas. Se muestra que las gráficas de $4 \mathrm{P}$ se enciman con la de $2 \mathrm{P}$ de una manera casi coincidente, la gráfica de $2 \mathrm{P}$ muestra más variación con la curva de $D=6(a / 2)$, pues es el caso más confinado.

En la figura 3.10 , mostramos las curvas de la presión sobre la placa $2, P_{2}$, como función de $t_{1}$, para un electrolito $2: 2,0.5 M$ y carga superficial en las paredes de $\sigma=0.272 \mathrm{C} / \mathrm{m}^{2}$, para varios valores de $D$. Podemos observar como la fuerza decrece a medida que $t_{1}$ aumenta hasta alcanzar un valor mínimo, pero después la fuerza crece nuevamente hasta que $t_{1}$ alcanza su valor muy grande, es decir, cuando la placa interna y externa se juntan alcanzan un valor que 
corresponde a un valor parecido a la fuerza en valor absoluto sobre la placa 1 en la misma posición.

Todas las curvas, a medida que disminuye la distancia $t_{1}$, tienden a un valor constante cercano al cero; pero no lo hacen en la misma forma que para el caso monovalente, en cuyas curvas para distancias $D$ menores quedan por arriba que de las de $D$ más grandes.

Este es un efecto de las correlaciones de corto alcance de los iones. La presión de las $2 \mathrm{P}$ se representa en linea clara y punteada y vemos un parecido menor con las $4 \mathrm{P}$ que para el caso monovalente, sobre todo para $D=6(a / 2)$. Las fuerzas correspondientes en la placa 1 y la placa 2, para $D=6(a / 2)$. discrepan mucho con las 2P. Puede también observarse que las presiones con $D=7(a / 2)$ y $9(a / 2)$ cambian menos, esto nos indica que a mayor confinamiento en la región 1 y en la región 2 , la presión cambia de manera importante con respecto a las $2 \mathrm{P}$.

En la figura 3.11 se muestran las curvas de $P_{1}$ y $P_{2}$ como función de $t_{1}$, para las condiciones de $2: 2,0.5 M$ y carga superficial de $0.272 \mathrm{C} / \mathrm{m}^{2}$ en las paredes, y para el caso de $D=13(\mathrm{a} / 2)$. En estas curvas, podemos observar los puntos donde las fuerzas netas sobre las dos placas coinciden, sin embargo, lo más interesante es que es un punto de equilibrio donde las dos presiones son cero y coinciden exactamente en esa distancia $t_{1}$.

Este punto en el cual ambas fuerzas tienen fuerzas netas igual a cero, es un estado de equilibrio. En las demás distancias $D$ no se encontró un punto tan exacto, sin embargo para las mismas condiciones del electrolito, en algunas distancias $t_{1}$ se aproximan mucho las fuerzas a cero.

Para el caso monovalente no se encontró ningún punto con la exactitud antes inencionada, y los puntos donde las dos fuerzas coinciden son más distantes del valor de la fuerza neta cero que para los casos divalente. Este resultado explica, porque la sustitución de iones monovalentes por iones divalentes tienden a estabilizar a las arcillas laminares, ya que mientras para el caso iones divalentes existe un punto de equilibrio en donde las placas permnecen a una distancia corta relativamente, en el caso monovalente las placas tienden a alejarse más lo que promueve la absorción de agua y puede llegar a promover el rompimiento de la estructura laminar.

En las figuras $3.12,3.13,3.14$ y 3.15 se presentan las gráficas de los valores de las cargas totales inducidas $\sigma_{1}, \sigma_{2}$ y $\sigma_{3}$, como función de $t_{1}$, en sus respectivas regiones. Las cargas inducidas se muestran con signo positivo.

La fig. 3.12, muestra las gráficas de la carga inducida por las paredes de las placas interiores en la región $I$, en función de la distancia $t_{1}$. para diferentes distancias $D$. Las curvas corresponden al caso divalente y monovalente que están representadas en la misma figura. Para el caso divalente cuando $t_{1}$ es pequeño (las placas interiores están muy cercanas), las partículas están muy confinadas en esa región, la concentración de partículas y su carga hacen que queden atrapadas por las paredes a pesar de haber poco espacio, hay una carga inducida mavor a la que compensaría una sola pared, esto muestra una sobrecarga en esta región. A medida que las placas interiores se separan. la carga en la región 1 disminuve, compensa a la carga de las paredes, y finalmerte cuando las placas internas alcanzan su mayor separación, sigue disminuyendo la carga inducida y es menor que en las paredes.

Se puede observar que cuando $t_{1}$ aumenta, casi al final, hay un ligero aumento de la carga inducida, porque la pared del otro lado de la placa que queda en la región II induce carga al otro lado en la región I. 
Las curvas para este caso oscilan para distancias $D$ mayores de $6 a / 2$, son decrecientes hasta un valor de la carga de aproximadamente de $0.206 \mathrm{C} / \mathrm{m}^{2}$ y vuelven a crecer todas las curvas a una carga aproximada de $0.212 C / \mathrm{m}^{2}$ (para valores de $t_{1}=2.89,5.89,9.89(a / 2)$ ). Para el caso más confinado $D=6(a / 2)$, la curva decrece sin puntos de retorno (no oscila) y cuando las placas se acercan o separan mucho se comporta como todas las curvas de $\sigma_{1}$, tiene un máximo y un mínimo.

En particular para $D=6(a / 2)$ la conducta es muy diferente al modelo de las dos placas, además $\sigma_{1}$ muestra menores valores que para $D=9(a / 2)$ y $13(a / 2)$, esto se debe al alto confinamiento producido por las placas externas, y es indicativo de la correlación que existe entre los diferentes compartimentos entre las placas. Analizando $\sigma_{1}$ para el caso monovalente podemos ver que cuando las placas interiores están muy cerca, la carga inducida es menor que en el caso de 2P: existen menos partículas en ese espacio y al separar las las placas aumenta el número de particulas, y se compensa la carga inducida con la carga de las paredes.

Si se separan más las placas 1 , hasta que $t_{1}$ alcanza su valor máximo, la carga inducida aumenta más alcanzando un sobrecargado de aproximadamente $0.15 \mathrm{C} / \mathrm{m}^{2}$ en la región I pues hay más particulas en ese espacio o pasan a esta región I, de la región II. (sólo estamos considerando la mitad de la región I). Las paredes exteriores están de alguna manera induciendo carga en la región I, cuando estén muy cercanas a las interiores. Las curvas de $\sigma_{1}$ son crecientes y no muestran oscilaciones. En los casos monovalente y divalente la carga total inducida del sistema de $2 \mathrm{P}$, se aproxima a las curvas correspondientes a las $4 \mathrm{P}$ en la región $I$ cuando $D$ es más grande $(D=13(a / 2))$. Para la distancia $D=13(a / 2)$ cuando $t_{1}$ es pequeño, las curvas se aproximan a la curva de $\sigma_{1}$ de las $2 \mathrm{P}$ simples.

Cuando $t_{1}$ es grande las curvas ya no se parecen por la influencia las placas externas, y la curva de dos placas se va a cero porque la interacción va disminuyendo. Para el caso monovalente la curva de $\sigma_{1}$ para las $2 \mathrm{P}$, está por debajo de las curvas de las $4 \mathrm{P}$, esto nos dice que hay más carga inducida en el modelo de las $4 \mathrm{P}$, en la región I que en el modelo de las 2P. Para el caso en que $D=6(a / 2)$ la curva de $\sigma_{1}$ en $t_{1} \rightarrow a / 2$ no se separa de las demás curvas como en el caso divalente, esto se debe a que muy confinado siguen induciéndose una cantidad de carga similar a la carga que se induce en las distancias $D=9$ y $13(a / 2)$.

En las figuras 3.13 y 3.14, se puede observar la carga total inducida en función de $t_{1}$ en la región II, para electrolitos $1: 1$ y $2: 2$ respectivamente. En el electrolito $2: 2$ cuando la placa 1 se acerca a la placa 2, todas las curvas tienen un ligero descenso por efecto de la carga de la pared del otro lado de la placa en la región III (ver fig. 3.2). Para este mismo caso los valores de la carga para $D=6 a / 2$ son crecientes y no muestran osclaciones, excepto cuando $t_{1}$ tiende a sus valores máximo y mínimo, mientras que sí son oscilatorias para $D=7$ y 9(a/2). Para 2:2, los valores de la carga inducida presentan un exceso de carga que supera la carga de las superficies de la placas 1 y 2 en contacto con esta regón del fluido cuando estas placas están muy cerca, y alcanzan un máximo de carga muy parecido, sin embargo, el máximo más alto se alcanza para menores valores de $D$, en este caso para $D=6 a / 2$.

Cuando las placas 1 y 2 están más separadas ( $t_{1}$ pequeño), la carga inducida disminựe por debajo de la carga equivalente de la placas 1 y la placa 2 . La carga total inducida la región II para el caso $2: 2$, sobrepasa a la carga de las placas en un amplio intervalo de $t_{1}$. En la región I y la región III (ver fig.3.15), las cargas inducidas están por debajo de la carga de las paredes en esas regiones en la mayor parte contrariamente a la región 11.

Esto indica que la carga salió de la región I y lII y pasó a la región II. En el caso 


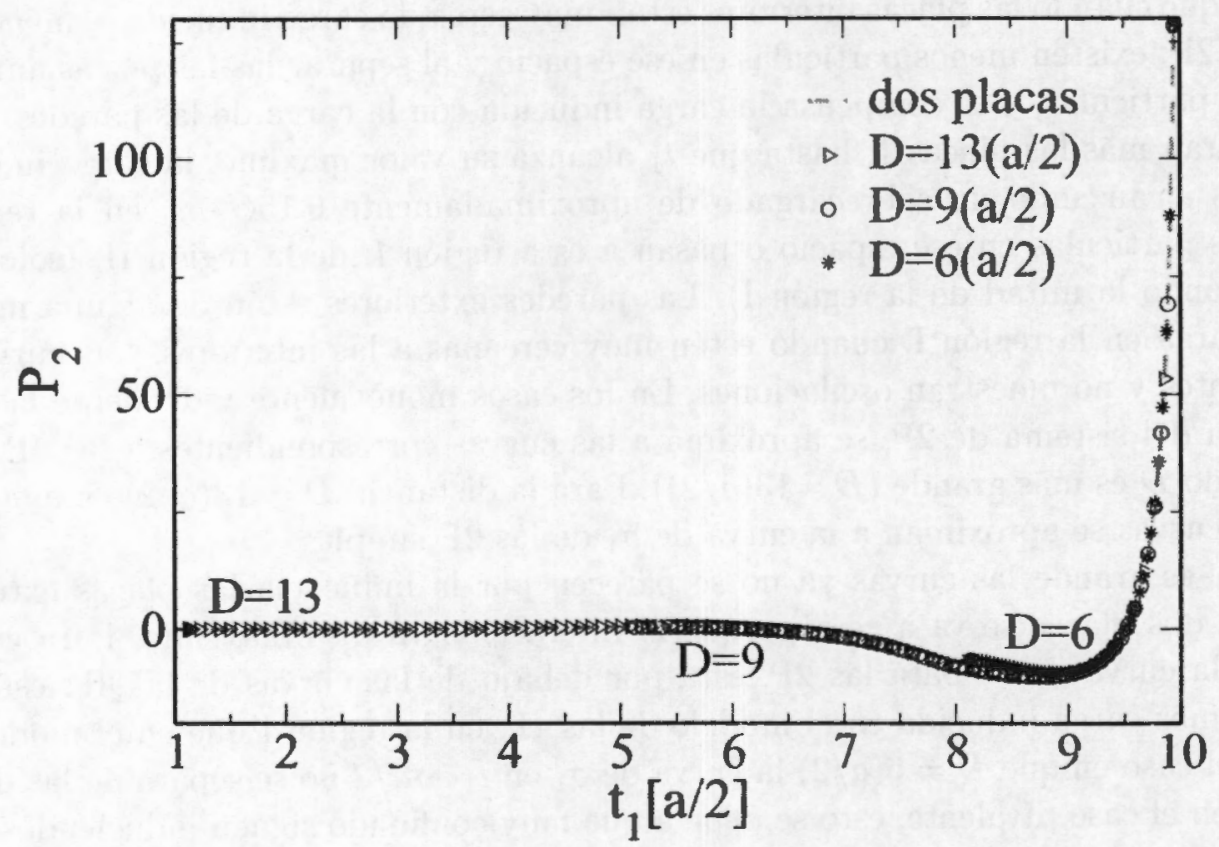

Figura 3.10: Gráfica que presenta los perfiles de presión neta sobre la placa 2, en función de la distancia entre el centro y la placa $1\left(t_{1}\right)$, las curvas corresponden a distancias diferentes del centro del sistema a la placa 2 , fijas, $D=6,9,13(a / 2)$, de izquierda a derecha. Para 2:2, $.5 \mathrm{M}, 0.272 \mathrm{C} / \mathrm{m}^{2}$. 


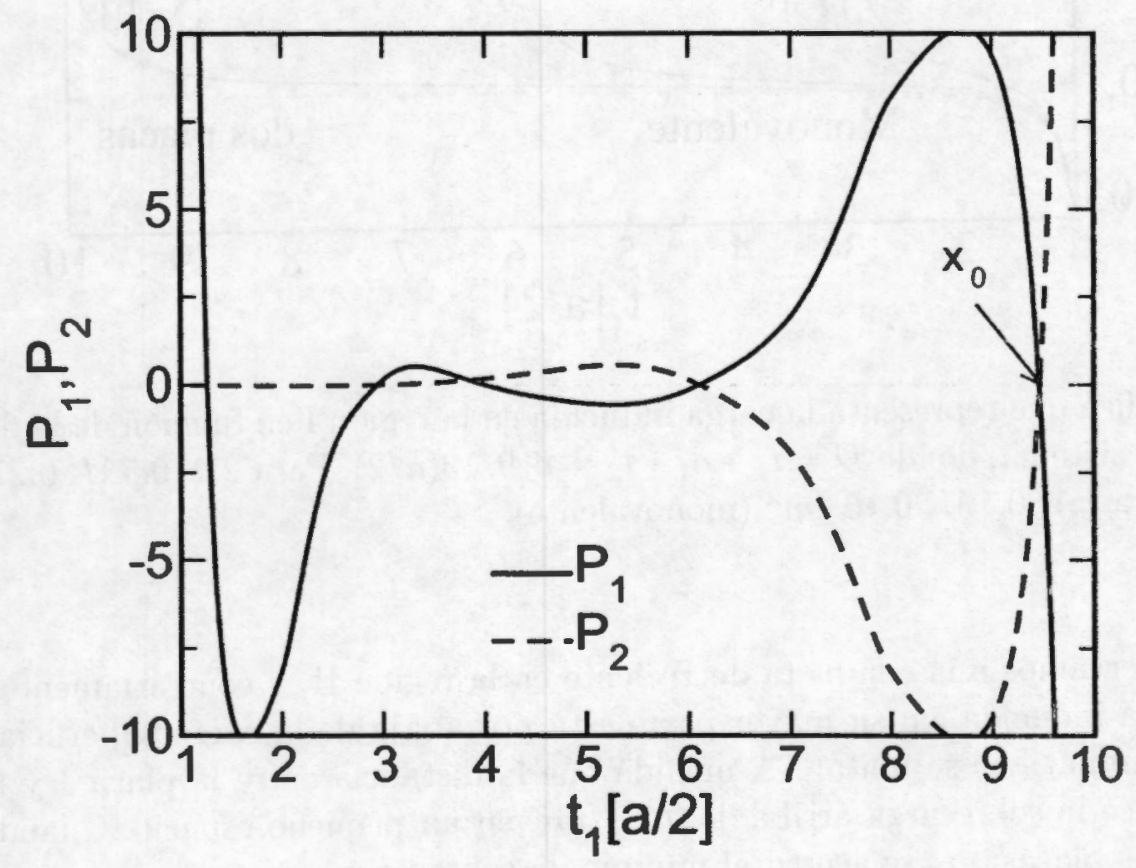

Figura 3.11: Gráfica que presenta la carga presión sobre la placa 1 y sobre la placa 2, en función de la distancia $t_{1}$, las lineas continuas y punteadass se indican en la misma figura, se ha mantenido fija la distancia $D=t_{1}+d_{1}+t_{2}=13(\mathrm{a} / 2)$. Para $2: 2, .5 \mathrm{M}, 0.272 \mathrm{C} / \mathrm{m}^{2}$. En $X_{0}$ corresponde a ambas fuerzas cero. 


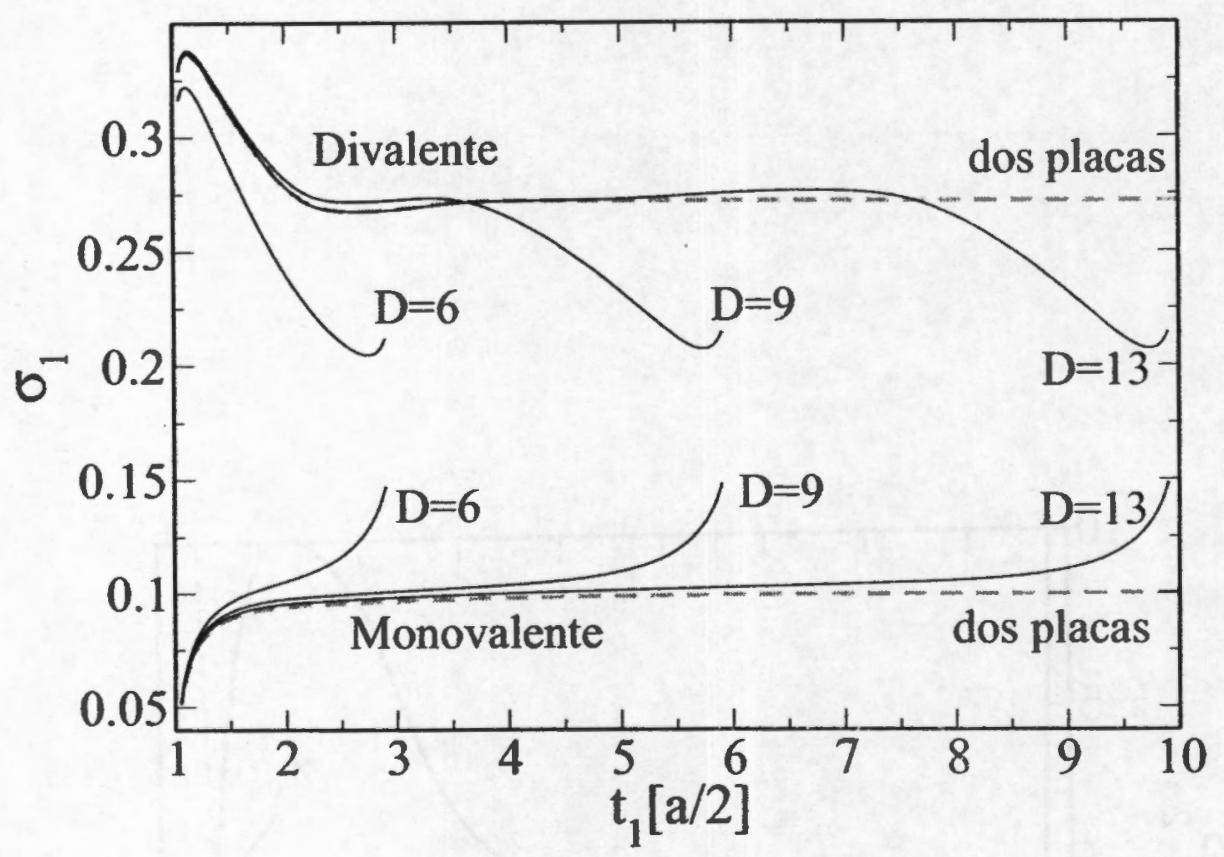

Figura 3.12: Gráfica que representa la carga inducida en la región I en función de la distancia $t_{1}$, del centro del sistema, donde $D=t_{1}+d_{1}+t_{2}=6,9,13(a / 2)$. Para 2:2, $0.5 M, 0.272 C / m^{2}$ (divalente) y para $1: 1,0.1 \mathrm{M}, 0.1 \mathrm{C} / \mathrm{m}^{2}$ (monovalente).

monovalente observamos una conducta decreciente en la región II, y contrariamente al caso divalente la carga inducida, en su mayor parte está por abajo de la carga superficial de las placas 1 y 2 , cuando estas se juntan. A medida que la distancia entre la placa 1 y la placa 2 aumenta aparece la sobrecarga arriba de $0.2 \mathrm{C} / \mathrm{m}^{2}$, en un pequeño espacio. Cuando están más separadas las placas o $t_{1}$ se acerca al mínimo, la sobrecarga es máxima.

En el caso monovalente se muestra que en general la carga inducida es menor a las paredes tanto en la región I como en la región II, pero en la región III (ver 3.15) la carga inducida excede a la carga de la pared. Entonces la carga de la región I y II pasa a la región III. En el caso monovalente cuando $t_{1}$ es más grande la carga inducida es la mitad de la carga de las dos paredes $\left(0.1 \mathrm{C} / \mathrm{m}^{2}\right)$, sigue habiendo menos carga inducida en los espacios más confinados, como en la región $\mathrm{I}$. Todas estas curvas son decrecientes y terminan hasta una carga aproximada de $0.1 \mathrm{C} / \mathrm{m}^{2}$, y no oscilan en ningún momento. Para 1:1, la carga inducida para el sistema de $2 \mathrm{P}$ se muestra con linea punteada y aparece por debajo de las curvas de las $4 \mathrm{P}$ en la región II. En esta región la curva para el sistema de las $2 \mathrm{P}$ cuando las placas están practicamente juntas, la carga total inducida se aproxima a $0.1 \mathrm{C} / \mathrm{m}^{2}$ como en las $4 \mathrm{P}$, que es la carga de una sola placa, y cuando las placas se alejan la carga inducida tiende al valor de $0.2 \mathrm{C} / \mathrm{m}^{2}$, porque es la carga que tienen las 2 placas. Las curvas en las 2 geometrías muestran diferencias, en particular para $D=6(a / 2)$ hay mavor discrepancia.

En la figura 3.15, se presentan las curvas de la carga inducida en la región III, vemos una conducta similar a la región 1, para los dos tipos de electrolito (1:1 y 2:2). Las curvas son decrecientes para 2:2 pasando por un mínimo y oscilan ligeramente para valores de 


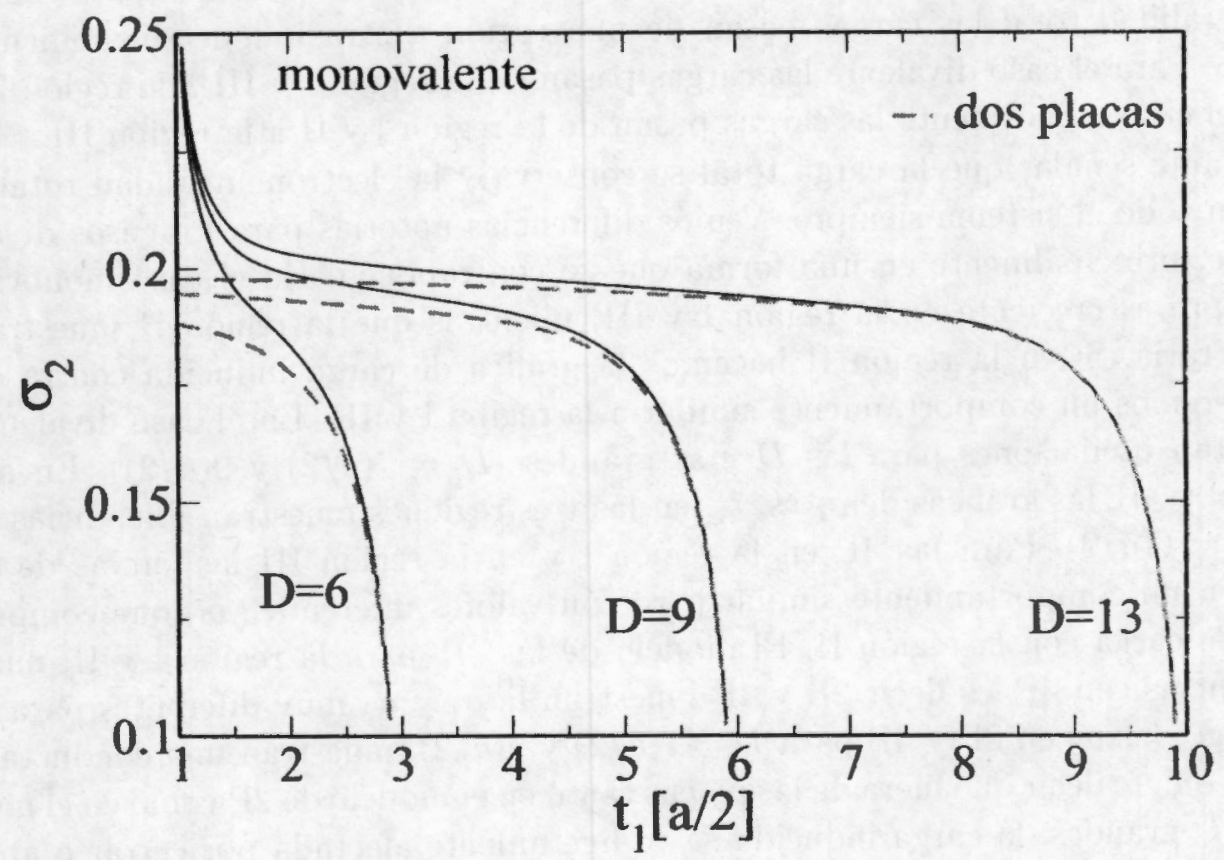

Figura 3.13: Gráfica que representa la carga inducida en la región ll en función de la distancia $t_{1}$, para diferentes valores de $D$, que están señalados en la figura. Las lineas obscuras corresponden a las cuatro placas. 
$D=7(a / 2)$ y $9(a / 2)$, sin embargo, menos que en la región I. Para este caso la carga inducida en los máximos rebasan ligeramente el valor de $0.272 \mathrm{C} / \mathrm{m}^{2}$, pero en general la carga inducida es menor a la carga de la placa exterior, es decir no hay subcargado. Las curvas en el modelo de las $2 \mathrm{P}$ muestran un mayor parecido con las $4 \mathrm{P}$, sin embargo, para $D=6 a / 2$ la diferencia es importante, aunque en el dibujo se nota poco, y son siempre menores que en 4P. Para 1:1 las curvas son crecientes en esta región, hasta que $t_{1}$ alcanza su valor máximo, con el valor aproximado de la carga de $0.1475 \mathrm{C} / \mathrm{m}^{2}$. Las cargas nunca son menores que la carga de una sola pared $\left(0.1 \mathrm{C} / \mathrm{m}^{2}\right)$, en todas las curvas hay un sobrecargado pues hay más carga que en una pared. En la región I la carga inducida no supera la carga de las 2 placas, las cargas salen de esta región y pasan a la región III. La carga inducida para las $2 \mathrm{P}$, en el caso monovalente en la región III es muy parecida a las 4P. Podemos observar que para conservar la electroneutralidad total las cargas pasan de una región a otra independientemente del confinamiento. Para el caso divalente las cargas pasan de la región I y III a la región II. Sin embargo, en el caso monovalente las cargas pasan de la región I y II a la región III.

Es importante señalar que la carga total se conserva y la electroneutralidad total debe permanecer en todo el sistema siempre. Vemos diferencias notorias para los casos divalente y monovalente, principalmente en una forma que se contraponen. En el caso monovalente la carga siempre es creciente en la región I y III, mientras que la región II muestra una conducta contraria. Si en la región II hacemos la gráfica de carga inducida contra $t_{2}$, en lugar de $t_{1}$, veremos un comportamiento similar a la región I y III. En el caso divalente las curvas presentan oscilaciones para las $D$ más grandes $(D=7(a / 2)$ y $9(a / 2))$. En ambas geometrias, $2 \mathrm{P}$ y $4 \mathrm{P}$, las gráficas de $\sigma_{i}$ vs. $t_{1}$, en las tres regiones muestran diferencias sobre todo para $D=6(a / 2)$. Para las $4 \mathrm{P}$ en la región I y en la región III las curvas de carga inducida tienen un comportamiento similar pero con valores diferentes, porque compensan la diferencia de carga con la región II. El modelo de las $2 \mathrm{P}$, para la región I y II, muestra muchas diferencias con $4 \mathrm{P}$, es decir $2 \mathrm{P}$ y $4 \mathrm{P}$ muestran la $\sigma_{1}$ y $\sigma_{2}$ muy diferentes para $1: 1$ y $2: 2$, sin embargo, las $\sigma_{3}$ en $2 \mathrm{P}$ y $4 \mathrm{P}$ para $D=7(a / 2)$ y $9(a / 2)$, muestran una conducta muy parecida. Esto quiere decir que fuera de las placas tanto en el modelo de $2 \mathrm{P}$ como en el modelo de $4 \mathrm{P}$ y para $D$ grandes, la carga inducida se ve ligeramente afectada por cerrar o abrir la separación de las placas. La $\sigma_{1}$ y $\sigma_{2}$ en las $4 \mathrm{P}$, muestran más variación con el movimiemto de las placas. Podemos observar que si en la región 1, para 1:1 o para 2:2 la carga inducida se incrementa entonces en la región II la carga inducida decrece, esto se debe a que la carga pasa de una región a otra y se reacomoda en la misma región, alcanzando el equilibrio, y conservando la condición de electroneutralidad total. Esto es un efecto de correlación entre los fluidos confinados donde las cargas inducidas se acomodan de una manera que mantengan el potencial químico constante, aún a costa de la violación de la electroneutralidad local.

\subsection{Conclusiones del capítulo 3}

Una principal conclusión de este capitulo es que se puede extender el problema de dos placas a cuatro placas de manera consistente.

Los resultados numéricos, y los resultados analíticos son consistentes con los resultados del sistema de dos placas $|79,80,22|$, en las condiciones apropiadas.

La condición general de electroneutralidad se satisface siempre, y se realizaron las gráficas 


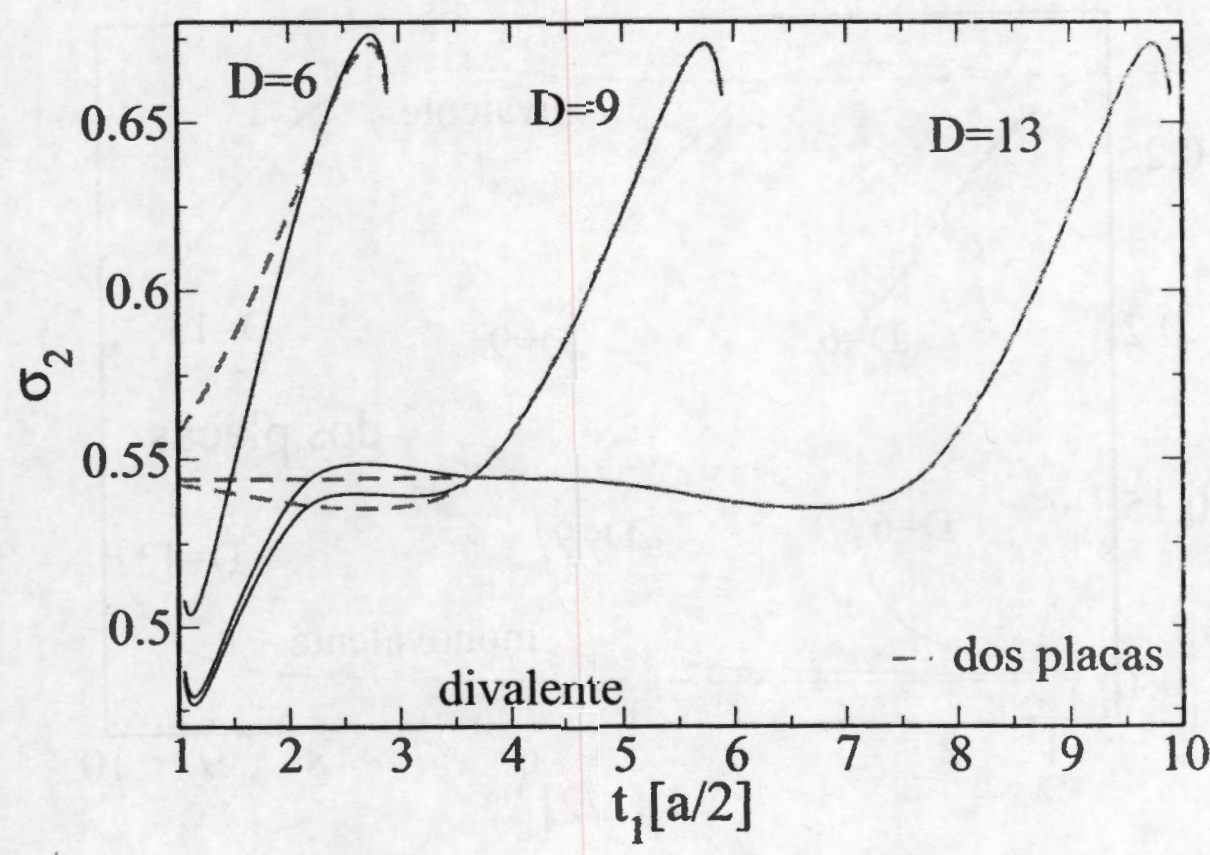

Figura 3.14: Gráfica que representa la carga inducida en la región II en función de la distancia $t_{1}$, para diferentes valores de $D$, que están señalados en la figura. Las lineas obscuras corresponden a las cuatro placas.

de una manera que se pudiera comparar con los resultados obtenidos de dos placas, i.e., en el caso límite donde la separación de las placas es muy grande el sistema se reduce a un sistema de una placa o de dos placas muy separadas.

Encontramos también que para distancias pequeñas entre las placas la condición de electroneutralidad local no se cumple $[180,181,182,9], \mathbf{y}$ pudo ser verificada en el caso de reducirse a dos placas cuando la distancia $t_{1}$ se hace muy grande por ejemplo.

Para el caso de los puntos de equilibrio en los cuales la fuerza es cero scbre las placas, depende de la separación entre las placas, de la carga sobre ellas y de la concentración del electrolito.

En este trabajo presentamos un caso en donde hallamos los puntos de equilibrio total. Podemos observar que el confinamiento generado por las placas externas sobre las placas internas produce efectos importantes en los diferentes compartimentos, pues entre más confinado esté el sistema, los resultados de $P_{1}, P_{2}, \sigma_{1}^{\prime}, \sigma_{2}^{\prime}, \sigma_{3}^{\prime}$ cambian de manera importante.

La adsorción de contraiones sobre la pared de la placa en algunos casos produce un efecto de mayor carga adsorvida que en las placas y se observa que cuando la carga sobre la pared aumenta, también este efecto. En cuanto a la concentración de contraiones entre las placas muestra que es mayor para el caso monovalente.

Para 2:2 el comportamiento oscilatorio, se manifiesta en las propiedades ta!'s como las fuerzas que son atractivas y repulsivas en varios intervalos, para diferentes distancias entre las placas. La forma que escogimos para calcular las fuerzas en las placas es consistente con los resultados de dos placas. Podemos también observar que la valencia de los iones afecta la 


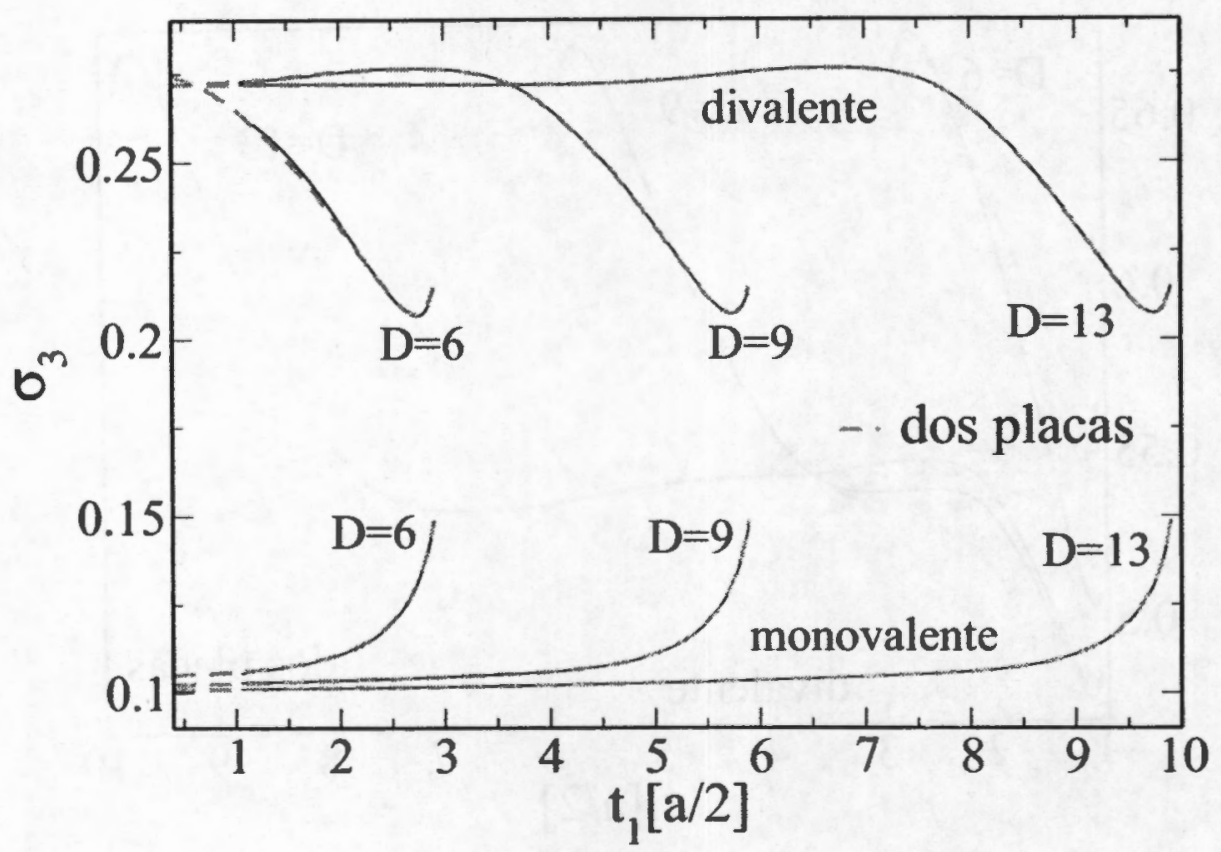

Figura 3.15: Gráfica que representa la carga inducida en la región 111 en función de la distancia $t_{1}$, para los casos monovalente y divalente indicados en la figura, para diferentes valores de $D$, que están señalados en la figura. Las lineas obscuras corresponden a las cuatro placas.

interacción entre las placas observando que para electrolitos 1:1, las fuerzas grandes tienen mayor alcance.

La interacción entre las dos placas internas da resultados diferentes comparados con la interacción entre una placa interna y una placa externa. Los perfiles de concentración de iones positivos y negativos en los puntos donde las presiones de ambas placas son cero o se aproximan a cero, muestran muy pocos coiones en la región $2\left(g_{+}(x) \approx 0\right)$ sobre todo cerca de las paredes, que corresponden a distancias intermedias y grandes que son $t_{1}=9.4649(a / 2)$ y $t_{1}=6.1107(a / 2)$, con $D=13(a / 2)$.

Para el punto donde $P_{1}=P_{2}=0$ en $t_{1}=9.4649(a / 2)$, los perfiles de concentración de contraiones en la región 1 y la región 3 , son simétricosy tienen valores menores de concentración que para las demás distancias $t_{1}$. Los perfiles de concentración de contraiones para $t_{1}=9.4649(a / 2)$, muestran en la región 1 y en la región 3 distribuciones simétricas y en la parte más cercana a las paredes tienen los mismos valores, estos valores sumados corresponden exactamente al valor mínimo de los contraiones en la región 2, en la parte central. 
Este trabajo es original en las siguientes partes de la tesis:

-La comparación de las dos geometrías, en el capítulo 2, y en la solución de las propiedades electrocinéticas para el cilindro con la teoría HNC/MSA.

-EI problema presentado en el capítulo 3, la teoría desarrollada por la teoría HNC/MSA, y el programa computacional para la solución del problema, así como los resultados obtenidos.

\subsection{Conclusiones Generales}

Conclusiones Generales

Conclusiones del Capítulo dos:

Después de haber analizado los cálculos realizados de las propiedades electrocinéticas con las teorías HNC/MSA y PB, con la condición de potencial zeta constante sobre las paredes de los dos nanoporos de diferente geometría inmersos en un electrolito, nos han permitido entender que:

La geometría de sistemas de nanoporos cargados de forma cilíndrica y de rendija de dos placas, influye en las propiedades electrocinéticas de fluidos cargados, que están confinados dentro de estos poros. Para un electrolito: 2:2 contenido en las dos geometrías a potencial zeta constante en las paredes se encuentra que existen valores del potencial para los cuales no convergen algunas propiedades electrocinéticas como es el flujo volumétrico. Las propiedades electrocinéticas para electrolitos monovalente y divalentes fueron realizados con condiciones diferentes en las paredes y los resultados fueron distintos con otra interpretación. Los resultados numéricos muestran que los efectos de confinamiento para los iones y las correlaciones de corto alcance son de mayor tamaño para el poro de las dos placas, con la teoría HNC/MSA. La comparación de los resultados de las teorías HNC/MSA y PB en las dos geometrías muestran ser mayores en la teoría HNC/MSA.

Para el caso de densidad de carga superficial constante sobre las paredes de los poros, se puede observar que:

Los potenciales electrostáticos pueden ser negativos para el caso divalente. La concentración promedio reducida de contraiones y coiones aumenta a medida que aumentamos la carga superficial sobre las paredes. El flujo de volumen reducido y redefinido aumenta con la carga. La conductancia capilar muestra importantes diferencias comparadas con el caso de potencial zeta constante pues para carga $0.272 \mathrm{C} / \mathrm{m}^{2}$ el potencial es muy grande. Para densidad de carga constante se pude observar una selectividad de la carga dentro de los poros, lo que nos permitiría separar las cargas.

Conclusiones del Capítulo tres:

Después de observar los perfiles de concentración de las cuatro placas, cuando acercamos más las placas exteriores, se ve que se separan más de los perfiles de concentración de las dos placas.

Entonces, en el sistema de cuatro placas, el confinamiento es importante porque nuestra diferencias notorias con las dos placas.

La condición de electroneutralidad total se satisface siempre en todos los casos.

En el caso límite donde la separación de las placas es muy grande el sistema se reduce a un sistema de una placa o de dos placas muy separadas, y los resultados son consistentes con estos sistemas. 
Encontramos que para distancias pequeñas entre las placas la condición de electroneutralidad local no se cumple siendo también consistente con las dos placas.

Existen configuraciones en las cuatro placas donde hay puntos de equilibrio en los cuales las fuerzas sobre las placas son cero, sólo para el caso divalente. Para el caso monovalente, no se observó este fenómeno por consiguiente creemos que sólo aparece para electrolitos divalentes. En este trabajo presentamos un caso en donde encontramos los puntos de equilibrio total.

La adsorción de contraiones sobre la pared de las placas, en algunos casos sobre compensan, la carga que existe sobre las paredes (sobrecargado). Este efecto, se nota más en electrolitos divalentes. Se observa que cuando la carga sobre la pared aumenta, también aumenta este efecto.

La concentración de contraiones entre las placas muestra en las gráficas que es mayor para el caso monovalente. Para el caso divalente el comportamiento oscilatorio se manifiesta en las propiedades tales como las fuerzas que son atractivas y repulsivas en varios intervalos para diferentes distancias entre las placas exteriores. También para estos electrolitos, cuando las placas están muy cercanas, las fuerzas son mayores que para el caso divalente. Pero, sin embargo, para los electrolitos monovalentes las fuerzas tienen mayor alcance. 


\subsection{Perspectivas}

Después de haber realizado el presente trabajo, se van a señalar los siguientes puntos en los que se podrían aplicar estos estudios:

1. Se iniciará por indicar, que los nanoporos de carbón se pueden usar como electrodos para la construcción de capacitores que contienen una doble capa eléctrica (EDL) y tienen el propósito de almacenar energía como una batería.

2. Otra aplicación práctica del fenómeno de la adsorción de iones sobre superficies cargadas, es el hecho de poder utilizarse, en procesos de separación de partículas para la purificación de agua y desalinización de líquidos.

3. Además, se puede mencionar el diseño de procesos que consisten en un transporte selectivo de iones, tales como canales a través de membranas biológicas, para la distribución de iones, del exterior de una célula, al interior de ésta, o viceversa.

4. Dentro de la industria petrolera existen diversas aplicaciones, se van a enumerar algunas que son de gran relevancia:

a) Se puede utilizar en la producción de petróleo, a través de la hidratación de los suelos arcillosos que contienen los yacimientos, evitando la disociación de las estructuras laminares de las arcillas.

b) Además, se podría aplicar en la producción de catalizadores para los combustibles obtenidos o derivados de los hidrocarburos, en los procesos de refinación de los combustibles.

5. Por último, debemos resaltar que el presente estudio se puede aplicar dentro de la industria del cemento:

a) En la elaboración de diversos tipos de cemento, combinando diferentes tipos de arcilla, que reaccionan de distinta manera al entrar en contacto con el agua.

b) En la producción de catalizadores para el endurecimiento del cemento, aplicando sales con iones divalentes, que evitan la disociación de las placas que conforman las arcillas. 


\section{Parte I}

Apéndice del Capítulo 2 


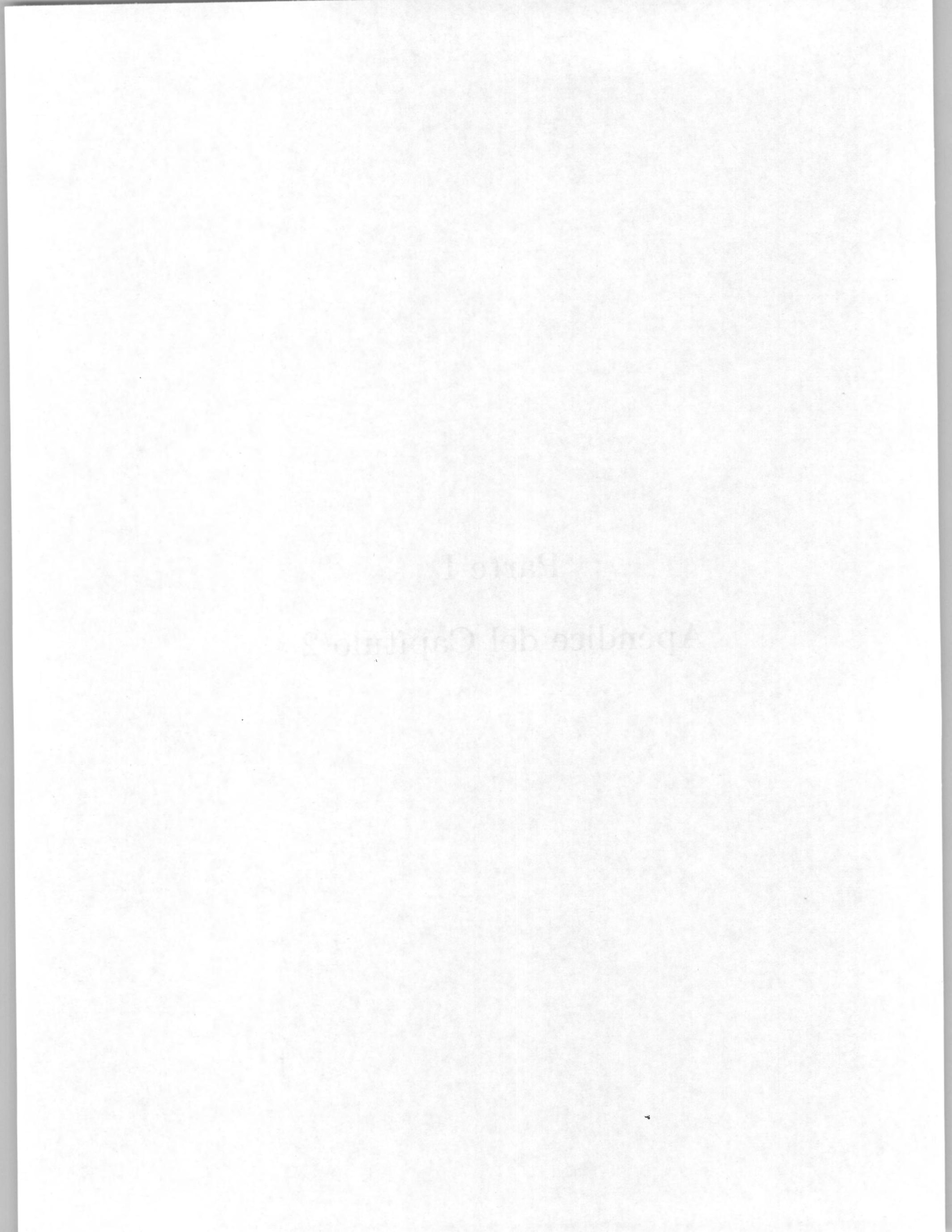


Las funciones de correlación de corto alcance para un electrolito RPM en la teoría MSA; en la Ec.2.5, $c_{s}(s)$ y $c_{d}^{s r}(s)$, están dadas por

$$
\begin{gathered}
c_{s}(s)=-c_{1}-6 \eta c_{2} s-\frac{1}{2} \eta c_{3} s^{3} ; \quad s \leq a \\
c_{d}^{s r}(s)=\frac{e^{2} \beta}{\epsilon s}\left\{1-\frac{2 \Gamma s}{(1+\Gamma a)}+\frac{\Gamma^{2} s^{2}}{(1+\Gamma a)^{2}}\right\} ; \quad s \leq a
\end{gathered}
$$

tal que $c_{s}(s)=c_{d}^{s r}(s)=0$ para $s>a$ y donde

$$
\begin{gathered}
\eta=\frac{1}{6} \pi a^{3} \sum_{m=1}^{n} \rho_{m} \\
c_{1}=\frac{(1+2 \eta)^{2}}{(1-\eta)^{4}} \\
c_{2}=\frac{(1+1 / 2 \eta)^{2}}{a(1-\eta)^{4}} \\
c_{3}=c_{1} / a^{3} \\
\Gamma a=-1 / 2+1 / 2(1+2 \kappa a)^{1 / 2} \\
\kappa^{2}=\frac{4 \pi \beta}{\epsilon} \sum_{m=1}^{n} \rho_{m} z_{m}^{2}
\end{gathered}
$$

La expresión para los kernels en términos de la integral de la cuación Ec. 2.19, están dados por

$$
\begin{aligned}
K_{c}(x, y) & =-4 y \int_{0}^{\phi_{0}}\left\{c_{1} \int_{0}^{z_{0}} d z+6 \eta c_{2} \int_{0}^{z_{0}} s d z\right. \\
& \left.+\frac{1}{2} \eta c_{3} \int_{0}^{z_{0}} s^{3} d z\right\} d \phi
\end{aligned}
$$

para $x-a \leq y \leq x+a . K(x, y)=0$ para $y>x+a$ y $y<x-a$.

$$
\begin{aligned}
L_{c}(x, y) & =\frac{4 y e^{2} \beta}{\epsilon} \int_{0}^{\phi_{0}}\left\{\int_{0}^{z_{0}} \frac{1}{s} d z\right. \\
& \left.-\frac{2 \Gamma}{(1+\Gamma a)} \int_{0}^{z_{0}} d z+\frac{\Gamma^{2}}{(1+\Gamma a)^{2}} \int_{0}^{z_{0}} s d z\right\} d \phi
\end{aligned}
$$

para $x-a \leq y \leq x+a . L(x, y)=0$ para $y>x+a$ y $y<x-a$

$$
A_{c}^{\prime}(x)=-\rho \int_{t}^{x+a} K(x, y) d y
$$

para $t-a \leq x \leq t$. 


\section{Parte II}

Apéndice del Capítulo 3 


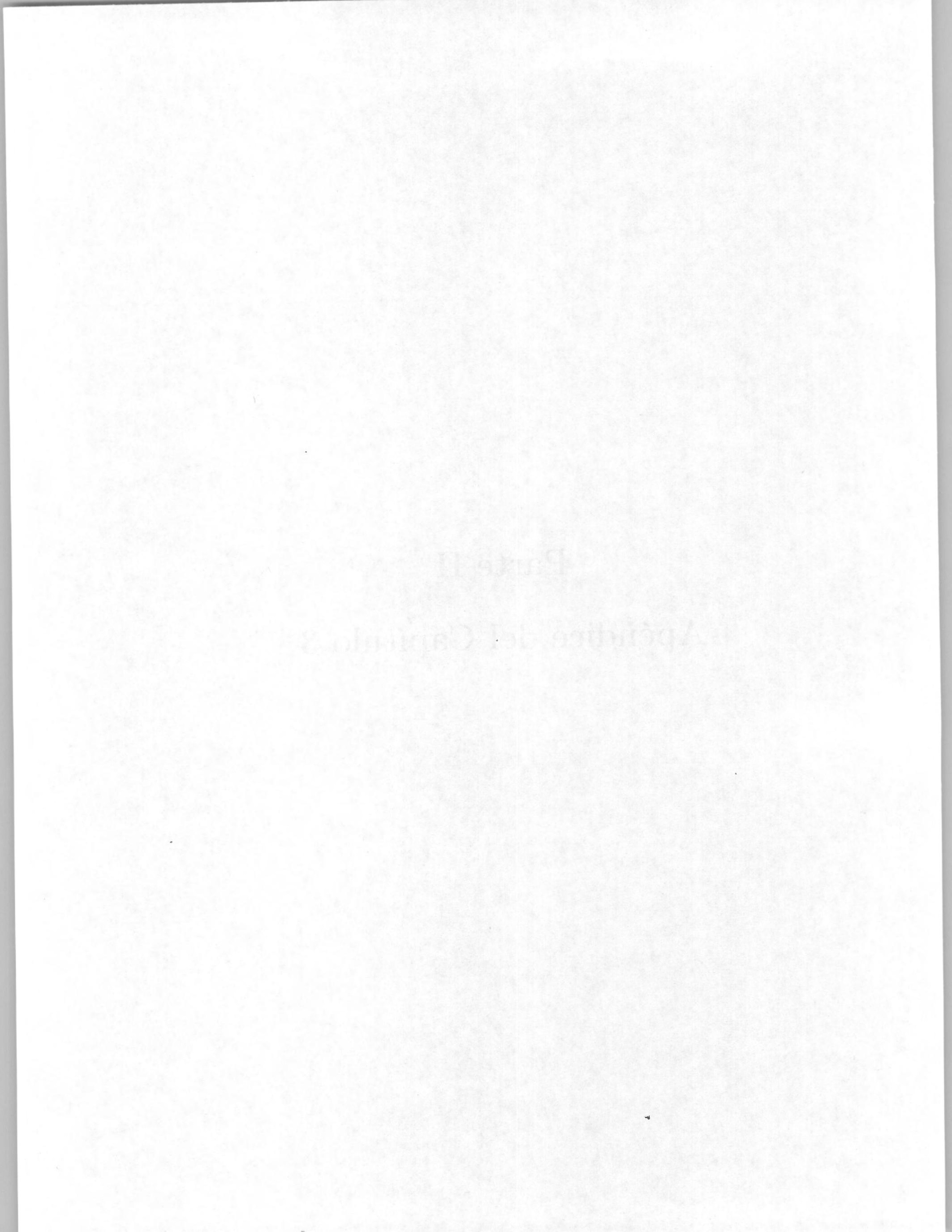




\section{Apéndice A}

\section{La función de distribución radial, para las Cuatro Placas}

A continuación se expresan los intervalos donde están definidas las integrales.

$$
\begin{aligned}
& (-\infty, \infty)=\left(-\infty,-t_{1}-d_{1} t_{2}-d_{2}-a / 2\right] \\
& \cup\left[-t_{1}-d_{1}-t_{2}-d_{2}-a / 2,-t_{1}-d_{1}-t_{2}+a / 2\right] \\
& \cup\left[-t_{1}-d_{1}-t_{2}+a / 2,-t_{1}-d_{1}-a / 2\right] \cup\left[-t_{1}-d_{1}-a / 2,-t_{1}+a / 2\right] \\
& \cup\left[-t_{1}+a / 2,0\right] \cup\left[0, t_{1}-a / 2\right] \cup\left[t_{1}-a / 2, t_{1}+d_{1}+a / 2\right] \\
& \cup\left[t_{1}+d_{1}+a / 2, t_{1}+d_{1}+t_{2}-a / 2\right] \\
& \cup\left[t_{1}+d_{1}+t_{2}-a / 2, t_{1}+d_{1}+t_{2}+d_{2}+a / 2\right] \\
& \cup\left[t_{1}+d_{1}+t_{2}+d_{2}+a / 2, \infty\right)
\end{aligned}
$$

donde $d_{1}$ y $d_{2}$ son el ancho de la placas interior y exterior respectivamente, de la parte derecha y $d_{1}$ y $d_{2}$ son los anchos de las placas interior y exterior respectivamente, de la parte izquierda. $t_{1}$ es la distancia del centro en $x=0$, a la placa interior del lado derecho, $t_{1}$, es la distancia del centro $x=0$, a la placa interior del lado izquierdo, $t_{2}$ es la distancia entre la placa 1 y la placa 2, del lado derecho, y $t_{2}$ es la distancia entre la placa 1 y la placa 2 del lado izquierdo.

Reemplazando sds por $\mathrm{zdz}$, podemos escribir la función de correlación como

$$
g_{a i}(x)=g_{a i}^{(1)}(x)+g_{a i}^{(2)}(x)
$$

donde 


$$
\begin{aligned}
& g_{\alpha i}(x)=\exp \left\{\left(-\beta u_{a i}(x)\right)\right. \\
& +2 \pi \int_{t_{1}+d_{1}+t_{2}+d_{2}+a / 2}^{\infty}\left(\int_{|x-y|}^{\infty} s c_{s}(s) d s \rho_{\alpha s}(y) d y+\int_{x+y}^{\infty} s c_{s}(s) d s \rho_{\alpha s}(y) d y\right) \\
& +2 \pi \int_{t_{1}+d_{1}+t_{2}-a / 2}^{t_{1}+d_{1}+t_{2}+d_{2}+a / 2}\left(\int_{|x-y|}^{\infty} s c_{s}(s) d s \rho_{\alpha s}(y) d y+\int_{x+y}^{\infty} s c_{s}(s) d s \rho_{\alpha s}(y) d y\right) \\
& +2 \pi \int_{t_{1}+d_{1}+a / 2}^{t_{1}+d_{1}+t_{2}-a / 2}\left(\int_{|x-y|}^{\infty} s c_{s}(s) d s \rho_{\alpha s}(y) d y+\int_{x+y}^{\infty} s c_{s}(s) d s \rho_{\alpha s}(y) d y\right) \\
& +2 \pi \int_{t_{1}-a / 2}^{t_{1}+d_{1}+\alpha / 2}\left(\int_{|x-y|}^{\infty} s c_{s}(s) d s \rho_{\alpha s}(y) d y+\int_{x+y}^{\infty} s c_{s}(s) d s \rho_{\alpha s s}(y) d y\right) \\
& +2 \pi \int_{0}^{t_{1}-a / 2}\left(\int_{|x-y|}^{\infty} s c_{s}(s) d s \rho_{\alpha s}(y) d y+\int_{x+y}^{\infty} s c_{s}(s) d s \rho_{\alpha s}(y) d y\right) \\
& +2 \pi \int_{0}^{t_{1}-a / 2}\left(\int_{|x-y|}^{\infty} s c_{d}^{s r}(s) d s \rho_{\alpha d}(y) d y+\int_{x+y}^{\infty} s c_{d}^{s r}(s) d s \rho_{\alpha d}(y) d y\right) \\
& +2 \pi \int_{t_{1}+d_{1}+a / 2}^{t_{1}+d_{1}+t_{2}-a / 2}\left(\int_{|x-y|}^{\infty} s c_{d}^{s r}(s) d s \rho_{\alpha d}(y) d y+\int_{x+y}^{\infty} s c_{d}^{s r}(s) d s \rho_{\alpha d}(y) d y\right) \\
& +2 \pi \int_{t_{1}+d_{3}+t_{2}+d_{2}+a / 2}^{\infty}\left(\int_{|x-y|}^{\infty} s c_{d}^{s r}(s) d s \rho_{\alpha d d}(y) d y+\int_{x+y}^{\infty} s c_{d}^{s r}(s) d s \rho_{\alpha d l}(y) d y\right) \\
& +\frac{2 \pi \beta e^{2} z_{i}}{\varepsilon} \int_{t_{1}+d_{1}+t_{2}+d_{2}+a / 2}^{\infty}(x+y+|x-y|) \rho_{\alpha d}(y) d y \\
& +\frac{2 \pi \beta e^{2} z_{i}}{\varepsilon} \int_{t_{1}+d_{1}+a / 2}^{t_{1}+d_{1}+t_{2}-a / 2}(x+y+|x-y|) \rho_{\alpha d d}(y) d y \\
& \left.+\frac{2 \pi \beta e^{2} z_{i}}{\varepsilon} \int_{t_{1}+d_{3}+t_{2}+d_{2}+a / 2}^{\infty}(x+y+|x-y|) \rho_{\alpha d}(y) d y\right\}+O(\infty)
\end{aligned}
$$

para $x \geq 0, \quad y$ para la parte izquierda tenemos una ecuación similar. Para efecto de distinguir los intervalos escribimos las distancias de la izquierda primadas, donde hemos definido $t_{1}^{\prime}=t_{1}, t_{2}^{\prime}=t_{2}, d_{2}^{\prime}=d_{2}, d_{2}^{\prime}=d_{2}$ 


$$
\begin{aligned}
& g_{\alpha i}(x)=\exp \left\{\left(-\beta u_{\alpha i}(x)\right)\right. \\
& +2 \pi \int_{t_{1}^{\prime}+d_{1}^{\prime}+t_{2}^{\prime}+d_{2}^{\prime}+a / 2}^{\infty}\left(\int_{x+y}^{\infty} s c_{s}(s) d s \rho_{\alpha s}(y) d y+\int_{|x-y|}^{\infty} s c_{s}(s) d s \rho_{\alpha s}(y) d y\right) \\
& +2 \pi \int_{t_{1}^{\prime}+d_{1}^{\prime}+t_{2}^{\prime}-a / 2}^{t_{1}^{\prime}+d_{1}^{\prime}+t_{2}^{\prime}+d_{2}^{\prime}+a / 2}\left(\int_{x+y}^{\infty} s c_{s}(s) d s \rho_{\alpha s}(y) d y+\int_{|x-y|}^{\infty} s c_{s}(s) d s \rho_{\alpha s}(y) d y\right) \\
& +2 \pi \int_{t_{1}^{\prime}+d_{1}^{\prime}+a / 2}^{t_{1}^{\prime}+d_{1}^{\prime \prime}+t_{2}^{\prime}-a / 2}\left(\int_{x+y}^{\infty} s c_{s}(s) d s \rho_{\alpha s}(y) d y+\int_{|x-y|}^{\infty} s c_{s}(s) d s \rho_{\alpha s}(y) d y\right) \\
& +2 \pi \int_{t_{1}^{\prime}-a / 2}^{t_{1}^{\prime}+d_{1}^{\prime}+a / 2}\left(\int_{x+y}^{\infty} s c_{s}(s) d s \rho_{\alpha s}(y) d y+\int_{|x-y|}^{\infty} s c_{s}(s) d s \rho_{\alpha s}(y) d y\right) \\
& +2 \pi \int_{0}^{t_{1}^{\prime}-\alpha / 2}\left(\int_{x+y}^{\infty} s c_{s}(s) d s \rho_{\alpha s}(y) d y+\int_{|x-y|}^{\infty} s c_{z}(s) d s \rho_{\alpha s}(y) d y\right) \\
& +2 \pi \int_{0}^{t_{1}^{\prime}-\alpha / 2}\left(\int_{x+y}^{\infty} s c_{d}^{s r}(s) d s \rho_{\alpha d}(y) d y+\int_{|x-y|}^{\infty} s c_{d}^{s r}(s) d s \rho_{\alpha d}(y) d y\right. \\
& +2 \pi \int_{t_{1}^{\prime}+\alpha_{1}^{\prime}+a / 2}^{t_{1}^{\prime}+d_{1}^{\prime}+t_{2}^{\prime}-a / 2}\left(\int_{x+y}^{\infty} s c_{d}^{s r}(s) d s\right) \rho_{\alpha d}(y) d y+\int_{|x-y|}^{\infty} s c_{d}^{s r}(s) d s \rho_{a d}(y) d y \\
& +2 \pi \int_{t_{1}^{\prime}+d_{1}^{\prime}+t_{2}^{\prime}+d_{2}^{\prime}+a / 2}^{\infty}\left(\int_{x+y}^{\infty} s c_{d}^{s r}(s) d s\right) \rho_{o d}(y) d y+\int_{|x-y|}^{\infty} s c_{d}^{s r}(s) d s \rho_{a d}(y) d y \\
& +\frac{2 \pi \beta e^{2} z_{i}}{\varepsilon} \int_{t_{1}^{\prime}+d_{1}^{\prime}+t_{2}^{\prime}+d_{2}^{\prime}+a / 2}^{\infty}(x+y+|x-y|) \rho_{\text {add }}(y) d y \\
& +\frac{2 \pi \beta e^{2} z_{i}}{\varepsilon} \int_{t_{1}^{\prime}+d_{1}^{\prime}+a / 2}^{t_{1}^{\prime}+u_{1}^{\prime}+t_{2}^{\prime}-a / 2}(x+y+|x-y|) \rho_{o u d}(y) d y \\
& \left.+\frac{2 \pi \beta e^{2} z_{i}}{\varepsilon} \int_{t_{1}^{\prime}+t_{1}^{\prime}+l_{2}^{\prime}+d_{2}^{\prime}+a / 2}^{\infty}(x+y+|x-y|) \rho_{\alpha d}(y) d y\right\}+O(\infty)
\end{aligned}
$$

para $x \leq 0$

como el sistema es simétrico,

$$
g_{\alpha i}(y)=g_{\alpha i}(-y)
$$

el intervalo de definición se expresa como

$$
\begin{aligned}
& (0, \infty)=\left[0, t_{1}-a / 2\right] \cup\left[t_{1}-a / 2, t_{1}+d_{1}+a / 2\right] \\
& \cup\left[t_{1}+d_{1}+a / 2 . t_{1}+d_{1}+t_{2}-a / 2\right] \\
& \cup\left[t_{1}+d_{1}+t_{2}-a / 2, t_{1}+d_{1}+t_{2}+d_{2}+a / 2\right] \\
& \cup\left[t_{1}+d_{1}+t_{2}+d_{2}+a / 2, \infty\right)
\end{aligned}
$$

$\mathrm{El}$ potencial de interacción entre las moléculas $\alpha$ y los iones $\mathrm{i}, u_{\alpha i}(x)$ lo podemos separar en dos partes, un término de pared-esfera dura $u_{\alpha i}^{*}(x)$, y otro término de interacción electrostático $u_{\alpha i}^{\epsilon l}(x)$ donde

$$
\begin{aligned}
& -\beta u_{\alpha i}^{e l}(x)=\frac{\pi \beta e z_{i}}{\varepsilon}\left[\left(x+t_{1}+d_{1}+t_{2}+d_{2}-\left|x-t_{1}-d_{1}-t_{2}-d_{2}\right|\right) \sigma_{4}\right. \\
& \quad+\left(x+t_{1}+d_{1}+t_{2}-\left|x-t_{1}-d_{1}-t_{2}\right|\right) \sigma_{3} \\
& \quad+\left(x+t_{1}+d_{1}-\left|x-t_{1}-d_{1}\right|\right) \sigma_{2} \\
& \left.\quad+\left(x+t_{1}-\left|x-t_{1}\right|\right) \sigma_{1}\right]
\end{aligned}
$$

para 
106

$$
\begin{gathered}
0 \leq x \leq t_{1} \quad y / o t_{1}+d_{1} \leq x \quad y / o t_{1}+d_{1}+t_{2} \leq x \\
y / 0 t_{1}+d_{1}+t_{2}+d_{2} \leq x
\end{gathered}
$$

y para la parte izquierda, representados en forma primada, y las densidades de carga son iguales que en la parte derecha

$$
\begin{aligned}
& -\beta u_{\alpha i}^{e l}(x)=\frac{\pi \beta e z_{i}}{\varepsilon}\left[\left(-\left(x+t_{1}^{\prime}+d_{1}^{\prime}+t_{2}^{\prime}+d_{2}^{\prime}\right)-\left|-\left(x-t_{1}^{\prime}-d_{1}^{\prime}-t_{2}^{\prime}-d_{2}^{\prime}\right)\right|\right) \sigma_{4}\right. \\
& \quad+\left(-\left(x+t_{1}^{\prime}+d_{1}^{\prime}+t_{2}^{\prime}\right)-\left|-\left(x-t_{1}^{\prime}-d_{1}^{\prime}-t_{2}^{\prime}\right)\right|\right) \sigma_{3} \\
& \quad+\left(-\left(x+t_{1}^{\prime}+d_{1}^{\prime}\right)-\left|-\left(x-t_{1}^{\prime}-d_{1}^{\prime}\right)\right|\right) \sigma_{2} \\
& \left.\quad+\left(-\left(x+t_{1}^{\prime}\right)-\left|-\left(x-t_{1}^{\prime}\right)\right|\right) \sigma_{1}\right]
\end{aligned}
$$

para

$$
\begin{gathered}
0 \geq x \geq-t_{1} \quad y / 0-\left(t_{1}+d_{1}\right) \geq x \quad y / 0 \quad-\left(t_{1}+d_{1}+t_{2}\right) \geq x \\
y / o-\left(t_{1}+d_{1}+t_{2}+d_{2}\right) \geq x
\end{gathered}
$$

con $t_{1}=t_{1}^{\prime}, t_{2}=t_{2}^{\prime}, d_{1}=d_{1}^{\prime}$ y $d_{2}=d_{2}^{\prime}$, caso simétrico y

$$
\begin{aligned}
& -\beta u_{\alpha i}^{e l}(x)=\frac{\pi \beta e z_{i}}{\varepsilon}\left[\left(x+t_{1}+d_{1}+t_{2}+d_{2}-\left|x-t_{1}-d_{1}-t_{2}-d_{2}\right|\right)\left(\sigma_{4}+\sigma_{4}\right)\right. \\
& \quad+\left(x+t_{1}+d_{1}+t_{2}-\left|x-t_{1}-d_{1}-t_{2}\right|\right)\left(\sigma_{3}+\sigma_{3}\right) \\
& \quad+\left(x+t_{1}+d_{1}-\left|x-t_{1}-d_{1}\right|\right)\left(\sigma_{2}+\sigma_{2}\right) \\
& \left.\quad+\left(x+t_{1}-\left|x-t_{1}\right|\right)\left(\sigma_{1}+\sigma_{1}\right)\right] \\
& \\
& \quad-\beta u_{\alpha i}^{e l}(x)=\frac{2 \pi \beta e z_{i}}{\varepsilon}\left[\left(x+t_{1}+d_{1}+t_{2}+d_{2}-\left|x-t_{1}-d_{1}-t_{2}-d_{2}\right|\right) \sigma_{4}\right. \\
& \quad+\left(x+t_{1}+d_{1}+t_{2}-\left|x-t_{1}-d_{1}-t_{2}\right|\right) \sigma_{3} \\
& \quad+\left(x+t_{1}+d_{1}-\left|x-t_{1}-d_{1}\right|\right) \sigma_{2} \\
& \left.\quad+\left(x+t_{1}-\left|x-t_{1}\right|\right) \sigma_{1}\right]
\end{aligned}
$$




\section{Apéndice B}

\section{Desarrollo Analítico de los Kernels de la Función de Distribución Radial, para las Cuatro Placas}

La aproximación MSA

$$
c_{i m}(s)=c_{s}(s)+z_{i} z_{m} c_{d}^{s r}(s)-\beta z_{i} z_{m} / \varepsilon s \quad, s<a
$$

donde $c_{s}(s)$ y $c_{d}^{s r}(s)$ son iguales a cero para $s>a$

$$
c_{s}(s)=-c_{1}-6 \eta c_{2} s-\frac{1}{2} \eta c_{3} s^{3}
$$

y

$$
c_{d}^{s r}(s)=\frac{e^{2} \beta}{\varepsilon s}\left\{1-\frac{2 \Gamma}{(1+a)} s-\frac{\Gamma^{2}}{(1+\Gamma a)^{2}} s^{2}\right\}
$$

para $s \leq a$ y cero en otro caso; donde

$$
\begin{gathered}
c_{1}=\frac{(1+2 \eta)^{2}}{(1-\eta)^{4}} \\
c_{2}=\frac{\left(1+\frac{1}{2} \eta\right)^{2}}{a(1-\eta)^{4}} \\
c_{3}=\frac{1}{a^{3}} \\
\eta=\frac{1}{6} \pi a^{3} \sum_{l=1}^{n} \rho_{l} \\
\Gamma a=-\frac{1}{2}+\frac{1}{2}(1+2 \kappa a)^{1 / 2}
\end{gathered}
$$


108

$$
\kappa^{2}=\frac{4 \pi \beta e^{2}}{\varepsilon} \sum_{l=1}^{n} \rho_{l} z_{l}^{2}
$$

sustituyendo las ecs. (A1), (A2) en la ec.(10), tenemos

$$
\begin{aligned}
& K_{1}(x \cdot y)=\frac{1}{2} c_{1}\left[(x+y)^{2}-a^{2}\right]+\frac{2 \eta}{a} c_{2}\left[|x+y|-a^{3}\right] \\
& +\frac{\eta c_{1}}{10 a^{3}}\left[|x+y|^{5}-a^{5}\right]
\end{aligned}
$$

para $0 \leq x \leq a \quad$ y $\quad 0 \leq y \leq a-x$, donde

$$
\begin{gathered}
K(x, y) \equiv K_{1}(x, y)+K_{2}(x, y) \\
K_{1}(x, y) \equiv \int_{x+y}^{\infty} s c_{s}(s) d s \\
K_{2}(x, y) \equiv \int_{|x-y|}^{\infty} s c_{s}(s) d s \\
K_{2}(x, y)=K_{1}(x,-y)
\end{gathered}
$$

para $x-a \leq y \leq x+a$

y

$$
\begin{aligned}
& L_{1}(x, y)=\frac{e^{2} \beta}{\varepsilon}\left\{[a-|x+y|]-\frac{\Gamma}{(1+a \Gamma)}\left[a^{2}-\left((x+y)^{2}\right]\right.\right. \\
& \left.+\frac{1}{3}\left(\frac{\Gamma}{1+a \Gamma}\right)^{2}\left[a^{3}-|x+y|^{3}\right]\right\}
\end{aligned}
$$

$0 \leq x \leq a$ y $0 \leq y \leq a-x$

donde

$$
\begin{gathered}
L(x, y) \equiv L_{1}(x, y)+L_{2}(x, y) \\
L_{1}(x, y) \equiv \int_{x+y}^{\infty} s c_{d}^{s r}(s) d s \\
L_{2}(x, y) \equiv \int_{|x-y|}^{\infty} s c_{d}^{s r}(s) d s \\
L_{2}(x, y)=L_{1}(x,-y)
\end{gathered}
$$

para $\quad x-a \leq y \leq x+a . . \quad \mathrm{y}$

$k_{1}(x, y)=k_{2}(x, y)=L_{1}(x, y)=L_{2}(x, y)$, otro caso en los intervalos $\left[t_{1}-a / 2, t_{1}+d_{1}+a / 2\right] \quad y$

$\left[t_{1}+d_{1}+t_{2}-a / 2, t_{1}+d_{1}+t_{2}+d_{2}+a / 2\right]$, 


$$
\rho_{a s}(y)=-\sum_{m=1}^{n} \rho_{m} \equiv-\rho_{T}
$$

y

$$
\rho_{\text {add }}(y)=-\sum_{m=1}^{n} z_{m} \rho_{m}=0
$$

con ayuda de las Eqs.(B.10) y (B.14), las siguientes ecuaciones son obtenidas analíticamente,

$$
\begin{gathered}
j_{1}(x) \equiv-2 \pi \rho_{T} \int_{t_{1}-a / 2}^{t_{3}+d_{1}+a / 2} K_{1}(x, y) d y \\
j_{2}(x) \equiv-2 \pi \rho_{T} \int_{t_{1}-a / 2}^{t_{1}+d_{1}+a / 2} K_{2}(x, y) d y \\
j_{3}(x) \equiv-2 \pi \rho_{T} \int_{t_{1}-d_{1}+t_{2}-a / 2}^{t_{1}-d_{1}+t_{2}+d_{2}+a / 2} K_{1}(x, y) d y \\
j_{4}(x) \equiv-2 \pi \rho_{T} \int_{t_{1}-d_{1}+t_{2}-a / 2}^{t_{1}+d_{1}+t_{2}+d_{2}+u / 2} K_{2}(x, y) d y
\end{gathered}
$$

y pueden ser escritas como

$$
j_{1}(x)=f_{1}(x)
$$

para, $a / 2 \leq t_{1} \leq \frac{3}{2} a$ y $0 \leq x \leq \operatorname{mín}\left\{\frac{3}{2} a-t_{1} \cdot t_{1}-a / 2\right\}$ y cero en otro caso,

$$
j_{2}(x)=f_{1}(-x)
$$

para $a / 2 \leq t_{1}$ y máx $\left\{0, t_{1}-\frac{3}{2} a\right\} \leq x \leq t_{1}-a / 2$.

Las ecuación ecs. 3.7 y 3.6 fueron resueltas considerando $0 \leq x \leq t_{1}-a / 2$, donde

$$
\begin{aligned}
& f_{1}(x) \equiv 2 \pi \rho_{T}\left\{\frac { 1 } { 6 } c _ { 1 } \left[\left(t_{1}-a / 2+x\right)^{3}\right.\right. \\
& \left.-3 a^{2}\left(t_{1}-a / 2+x\right)+2 a^{3}\right]+\frac{\eta c_{2}}{2 a} \\
& \times\left[\left(t_{1}-a / 2+x\right)^{4}-4 a^{3}\left(t_{1}-a / 2+x\right)+3 a^{4}\right] \\
& +\frac{\eta c_{1}}{60 a^{3}}\left(t_{1}-a / 2+x\right)^{6} \\
& \left.\left.-6 a^{5}\left(t_{1}-a / 2+x\right)+5 a^{6}\right]\right\}
\end{aligned}
$$

Si $x \geq t_{1}+d_{1}+a / 2, \quad j_{1}(x)=0$ 


$$
\begin{aligned}
& j_{2}(x)=2 \pi \rho_{T}\left\{\frac{1}{6} c_{1}\left[\left(x-t_{1}-d_{1}-a / 2\right)^{3}-3 a^{2}\left(x-t_{1}-d_{1}-a / 2\right)+2 a^{3}\right]\right. \\
& +\frac{\eta c_{2}}{2 a}\left[\left(x-t_{1}-d_{1}-a / 2\right)^{4}-4 a^{3}\left(x-t_{1}-d_{1}-a / 2\right)+3 a^{4}\right] \\
& \left.+\frac{\eta c_{1}}{60 a^{3}}\left[\left(x-t_{1}-d_{1}-a / 2\right)^{6}-6 a^{5}\left(x-t_{1}-d_{1}-a / 2\right)+5 a^{6}\right]\right\}
\end{aligned}
$$

para $t_{1}+d_{1}+a / 2 \leq t_{1}+d_{1}+\frac{3}{2} a$ y cero en otro caso.

$$
j_{3}(x)=f_{2}(x)
$$

para $a / 2 \leq t_{1}+d_{1}+t_{2} \leq \frac{3}{2} a \quad$ y $\quad 0 \leq x \leq \min \left\{(3 / 2) a-t_{1}+d_{1}+t_{2}, t_{1}+d_{1}+t_{2}-a / 2\right\}$, y cero en otro caso,

$$
j_{4}(x)=f_{2}(-x)
$$

para $a / 2 \leq t_{1}+d_{1}+t_{2} \quad$ y $\quad \operatorname{máx}\left\{0, t_{1}+d_{1}+t_{2}-\frac{3}{2} a\right\} \leq t_{1}+d_{1}+t_{2}-a / 2$, Las ecuaciones (B29) y (B30) están definidas en $0 \leq x \leq t_{1}+d_{1}+t_{2}-a / 2$, donde

$$
\begin{aligned}
& f_{2}(x) \equiv 2 \pi \rho_{T}\left\{\frac { 1 } { 6 } c _ { 1 } \left[\left(t_{1}+d_{1}+t_{2}-a / 2+x\right)^{3}\right.\right. \\
& \left.-3 a^{3}\left(t_{1}+d_{1}+t_{2}-a / 2+x\right)^{3}+2 a^{3}\right]+\frac{\eta c_{2}}{2 a} \\
& \times\left[\left(t_{1}+d_{1}+t_{2}-a / 2+x\right)^{4}-4 a^{3}\left(t_{1}+d_{1}+t_{2}-a / 2+x\right)^{3}+3 a^{4}\right] \\
& \left.+\frac{\eta c_{1}}{60 a^{3}}\left[\left(t_{1}+d_{1}+t_{2}-a / 2+x\right)^{6}-6 a^{5}\left(t_{1}-a / 2+x\right)+5 a^{6}\right]\right\}
\end{aligned}
$$

si $x \geq t_{1}+d_{1}+t_{2}+d_{2}+a / 2, \quad j_{3}(x)=0$,

y

$$
\begin{aligned}
& j_{4}(x)=2 \pi \rho_{T}\left\{\frac { 1 } { 6 } c _ { 1 } \left[\left(x-t_{1}-d_{1}-t_{2}-d_{2}-a / 2\right)^{3}-\right.\right. \\
& \left.-3 a^{2}\left(x-t_{1}-d_{1}-t_{2}-d_{2}-a / 2\right)+2 a^{3}\right] \\
& +\frac{\eta c_{2}}{2 a}\left[\left(x-t_{1}-d_{1}-t_{2}-d_{2}-a / 2\right)^{4}\right. \\
& \left.-4 a^{3}\left(x-t_{1}-d_{1}-t_{2}-d_{2}-a / 2\right)+3 a^{4}\right] \\
& +\frac{\eta c_{1}}{60 a^{3}}\left[\left(x-t_{1}-d_{1}-t_{2}-d_{2}-a / 2\right)^{6}\right. \\
& \left.\left.-6 a^{5}\left(x-t_{1}-d_{1}-t_{2}-d_{2}-a / 2\right)+5 a^{6}\right]\right\}
\end{aligned}
$$

para $t_{1}+d_{1}+t_{2}+d_{2}+a / 2 \leq x \leq t_{1}+d_{1}+t_{2}+d_{2}+\frac{3}{2} a$ y cero en otro caso. 


\section{Apéndice C}

\section{La Fuerza sobre las placas}

En este apéndice definimos las densidades totales (ver fig.3.2), la densidad de carga superficial total del lado derecho es

$$
\sigma_{D}=\sigma_{1}+\sigma_{2}+\sigma_{3}+\sigma_{4}
$$

y la carga inducida total es

$$
\sigma_{D}^{\prime}=\sigma_{1}^{\prime}+\sigma_{2}^{\prime}+\sigma_{3}^{\prime}+\sigma_{4}^{\prime}
$$

donde en general tenemos $\sigma_{1} \neq \sigma_{1}^{\prime}, \sigma_{2}^{\prime} \neq \sigma_{2}^{\prime}, \sigma_{3} \neq \sigma_{3}^{\prime}, \sigma_{4} \neq \sigma_{4}^{\prime}$ y para el caso simétrico $\sigma_{D}=\sigma_{I}=\sigma_{T}$, donde $\sigma_{I}$ es la densidad de carga superficial total del lado izquierdo y $\sigma_{I}^{\prime}$ es la carga inducida total del lado izquierdo, tal que $\sigma_{D}^{\prime}=\sigma_{I}^{\prime}=\sigma_{T}^{\prime}$. La carga total en el sistema es $\sigma_{\text {total }}=2 \sigma_{T}$ y la carga total inducida es $\sigma_{\text {total }}^{\prime}=2 \sigma_{T}^{\prime}$ y se cumple la electroneutralidad total

$$
\sigma_{\text {total }}+\sigma_{\text {total }}^{\prime}=0
$$

La carga sobre la placa uno es $Q_{1}=\left(\sigma_{1}+\sigma_{2}\right) A$, donde $A$ es el área de la pared de la placa, la fuerza sobre la placa uno es

$$
F_{1}=\int d F_{1}=\int Q_{1} d E
$$

donde $d E=\frac{d \sigma(x)}{2 \epsilon}$ y $\sigma(x)$ es la densidad de carga superficial en la posición $x, F_{1}$ es la fuerza neta sobre la placa uno, esta fuerza es la suma de las fuerzas tanto de la parte izquierda como de la parte derecha de la placa (debemos considerar apropiadamente el signo de las fuerzas en cada lado de la placa) De manera similar, para la placa dos tenemos

$$
F_{2}=\int d F_{2}=\int Q_{2} d E
$$

donde

$$
Q_{2}=\left(\sigma_{3}+\sigma_{4}\right) A
$$

la carga inducida por las placas está definida como 
112

$$
\begin{gathered}
\sigma_{1}^{\prime}=e \int_{0}^{t_{1}-a / 2} \rho_{\alpha d}(y) d y \\
\sigma_{2}^{\prime}+\sigma_{3}^{\prime}=e \int_{t_{1}+d_{1}+a / 2}^{t_{1}+d_{1}+t_{2}-a / 2} \rho_{\alpha d}(y) d y \\
\sigma_{4}^{\prime}=e \int_{t_{1}+d_{1}+t_{2}+d_{2}+a / 2}^{\infty} \rho_{\alpha d}(y) d y
\end{gathered}
$$

el sistema de referencia fue elegido en $x=0$. La presión neta en la placa es $P=F / A$, donde $F$ es una fuerza neta en la placa. 


\section{Apéndice D}

\section{Ecuaciones para el Potencial Electrostático, para el caso de Las Cuatro Placas}

Considérese un sistema definido en un espacio infinito, donde por construcción sólo existe una dimensión que cambia, $x, y$ por simetría sólo tenemos el intervalo $[0, \infty)$ repartido en nueve regiones como se muestra en la figura 1 , para estas regiones $\psi_{i}(x)$ donde $i=1,2, . ., 9$; tiene diferentes expresiones. Para las regiones donde no existe carga el potencial electrostático está regido por la ecuación de Laplace, expresada como,

$$
\nabla^{2} \psi_{i}(\mathbf{r})=\mathbf{0}
$$

y para las regiones donde hay carga, el potencial cumple la ecuación de Poisson tal que

$$
\nabla^{2} \psi_{i}(\mathbf{r})=\frac{-4 \pi \mathbf{e}}{\varepsilon} \rho_{\alpha d}(\mathbf{r})
$$

en donde $\psi_{i}(x)$ es el potencial electrostático medio en la región i. Por la geometría del problema, podemos expresarlo en función de una sola variable

$$
\frac{d \psi_{i}(x)}{d x}=\frac{-4 \pi e}{\varepsilon} \rho_{\alpha d}(x)
$$

Analizando el potencial para las diferentes regiones se deducen de la siguiente manera:

1. Región 9: $l_{1} \equiv t_{1}+d_{1}+t_{2}+d_{2}+a / 2 \leq x$

Aplicando las condiciones de frontera siguientes:

$$
\begin{aligned}
& \lim _{x \rightarrow \infty} \psi_{9}(x)=0, \\
& \lim _{x \rightarrow \infty} \frac{d \psi_{9}(x)}{d x}=0 .
\end{aligned}
$$


tenemos que por la ec.(D.1),

$$
\frac{d \psi_{9}(x)}{d x}=\frac{4 \pi e}{\varepsilon} \int_{x}^{\infty} \rho_{\alpha d}(y) d y
$$

así,

$$
\psi_{9}(x)=\frac{-4 \pi e}{\varepsilon} \int_{x}^{\infty} d x^{\prime} \int_{x^{\prime}}^{\infty} \rho_{\text {ad }}(y) d y
$$

y encontramos que

$$
\psi_{9}(x)=\frac{4 \pi e}{\varepsilon} \int_{x}^{\infty}(x-y) \rho_{\alpha d}(y) d y
$$

para $\mathrm{x}=l_{1}, \varphi_{1} \equiv \psi_{9}\left(l_{1}\right)$, entonces

$$
\varphi_{1}=\frac{4 \pi e}{\varepsilon} \int_{l_{1}}^{\infty}\left(l_{1}-y\right) \rho_{\alpha d}(y) d y
$$

2. Región 8: $l_{2} \equiv t_{1}+d_{1}+t_{2}+d_{2} \leq x \leq l_{1}$

por condiciones de frontera tenemos lo siguiente

$$
\begin{gathered}
\psi_{8}\left(l_{1}\right) \equiv \varphi_{1} \\
\varepsilon\left(\frac{d \psi_{9}(x)}{d x}\right)_{l_{1}}-\varepsilon\left(\frac{d \psi_{8}(x)}{d x}\right)_{l_{1}}=0
\end{gathered}
$$

aplicandó la ec. (D.1),

$$
\frac{d \psi_{8}(x)}{d x}=\text { const }
$$

Integrando Ec.(D.11) y usando las ecs.(D.5), (D.8) y (D.10), obtenemos

$$
\psi_{8}(x)=\frac{4 \pi \epsilon}{\varepsilon} \int_{l_{1}}^{\infty}(x-y) \rho_{\alpha d}(y) d y
$$

redefiniendo, tenemos

$$
\varphi_{2}=\psi_{8}\left(l_{2}\right)=\frac{4 \pi e}{\varepsilon} \int_{l_{3}}^{\infty}\left(l_{2}-y\right) \rho_{\alpha d}(y) d y
$$

3. Región 7: $l_{3} \equiv t_{1}+d_{1}+t_{2} \leq x \leq l_{2}$ 
De nuevo aplicando condiciones de frontera

$$
\begin{gathered}
\psi_{7}\left(l_{2}\right) \equiv \varphi_{2} \\
\varepsilon\left(\frac{d \psi_{8}(x)}{d x}\right)_{l_{2}}-\varepsilon\left(\frac{d \psi_{7}(x)}{d x}\right)_{l_{2}}=-4 \pi \sigma_{4}
\end{gathered}
$$

de las ecs. (D.1), (D.12) y (D.15), tenemos que

$$
\psi_{7}(x)=\frac{4 \pi \sigma_{4}}{\varepsilon}\left(x-l_{2}\right)+\frac{4 \pi e}{\varepsilon} \int_{l_{1}}^{\infty}(x-y) \rho_{\alpha d}(y) d y
$$

así,

$$
\varphi_{3}=\psi_{7}\left(l_{3}\right)=-\frac{4 \pi \sigma_{4}}{\varepsilon} d_{2}+\frac{4 \pi e}{\varepsilon} \int_{l_{1}}^{\infty}\left(l_{3}-y\right) \rho_{\mathrm{ad}}(y) d y
$$

en las cuatro placas la condición de electroneutralidad la escribimos como

$$
\begin{gathered}
\sigma_{1}+\sigma_{2}+\sigma_{3}+\sigma_{4}+\sigma_{1}^{i z q}+\sigma_{2}^{i z q}+\sigma_{3}^{i z y}+\sigma_{4}^{i z q}=-e \int_{-\infty}^{\infty} \rho_{\alpha d}(y) d y \\
\sigma_{1}+\sigma_{2}+\sigma_{3}+\sigma_{4}+\sigma_{1}^{i z q}+ \\
\sigma_{2}^{i z q}+\sigma_{3}^{i z q}+\sigma_{4}^{i z q}=-e \int_{l_{1}}^{\infty} \rho_{\alpha d}(y) d y-e \int_{l_{5}}^{l_{4}} \rho_{\alpha d d}(y) d y \\
-e \int_{0}^{l_{B}} h_{\alpha d}(y) d y-e \int_{-l_{\beta}}^{0} \rho_{\alpha d}(y) d y \\
-e \int_{-l_{4}}^{-l_{5}} h_{\alpha d}(y) d y-e \int_{-\infty}^{\beta_{1}} \rho_{\alpha d}(y) d y
\end{gathered}
$$

Dividiendo en dos intervalos el dominio de definición $(-\infty, 0]$ y $\quad[0, \infty)$

$$
\begin{gathered}
\sigma_{1}+\sigma_{2}+\sigma_{3}+\sigma_{4}=-e \int_{l_{1}}^{\infty} \rho_{a d}(y) d y-e \int_{l_{5}}^{l_{4}} \rho_{a d}(y) d y-e \int_{0}^{l_{8}} \rho_{a d d}(y) d y \\
\sigma_{1}^{i z q}+\sigma_{2}^{i z q}+\sigma_{3}^{i z q}+\sigma_{4}^{i z q}=-e \int_{-l_{8}}^{0} \rho_{\alpha d}(y) d y-e \int_{-l_{4}}^{-i_{5}} \rho_{\alpha d}(y) d y-e \int_{-\infty}^{l_{1}} \rho_{a d}(y) d y
\end{gathered}
$$

Por la simetía $\rho_{\text {ad }}(y)=\rho_{\text {ad }}(-y)$ y $\sigma_{i}=\sigma_{i}^{i z y}$ tenemos que por las ecs. (D.13), (D.17) y (D.19), que

$$
\begin{gathered}
f_{3}=\varphi_{2}-\frac{4 \pi \sigma_{4}}{\varepsilon} d_{2}-\frac{4 \pi d_{2} \epsilon}{\varepsilon} \int_{l_{1}}^{\infty} \rho_{\alpha u t}(y) d y \\
\varphi_{3}=\varphi_{2}-\frac{4 \pi d_{2}}{\varepsilon}\left(\sigma_{1}+\sigma_{2}+\sigma_{3}\right)-\frac{4 \pi d_{2} e}{\varepsilon} \int_{l_{5}}^{l_{4}} \rho_{\alpha d}(y) d y \\
-\frac{4 \pi d_{2} \epsilon}{\varepsilon} \int_{0}^{l_{8}} \rho_{\alpha d}(y) d y
\end{gathered}
$$


para $[0, \infty)$, además, para el caso particular de $\varphi_{2}=\varphi_{3}$, entonces

$$
\sigma_{4}=-e \int_{l_{1}}^{\infty} \rho_{\alpha d}(y) d y
$$

y

$$
\sigma_{1}+\sigma_{2}+\sigma_{3}=-e \int_{l_{5}}^{l_{4}} \rho_{\text {ad }}(y) d y-e \int_{0}^{l_{3}} \rho_{\text {aud }}(y) d y
$$

4. Región 6: $l_{4} \equiv t_{1}+d_{1}+t_{2}-a / 2 \leq x \leq l_{3}$

de manera similar que en las regiones anteriores y usando condiciones de frontera

$$
\psi_{6}\left(l_{3}\right) \equiv \varphi_{3}
$$

y

$$
\varepsilon\left(\frac{d \psi_{7}(x)}{d x}\right)_{l_{3}}-\varepsilon\left(\frac{d \psi_{6}(x)}{d x}\right)_{l_{3}}=-4 \pi \sigma_{3}
$$

tomando las ecs. (D.1), (D.16), D.20, C.9 y (D.25),

$$
\left(\frac{d \psi_{6}(x)}{d x}\right)_{l_{3}}=\frac{4 \pi}{\varepsilon} \sigma_{3}+\frac{4 \pi}{\varepsilon} \sigma_{4}+\frac{4 \pi e}{\varepsilon} \int_{l_{1}}^{\infty} \rho_{\alpha d}(y) d y
$$

integrando la ec. anterior y usando la ec.(D.24),

$$
\psi_{6}(x)=-\frac{4 \pi}{\varepsilon} \sigma_{4} d_{2}+\frac{4 \pi}{\varepsilon}\left(\sigma_{3}+\sigma_{4}\right)\left(x-l_{3}\right)+\frac{4 \pi e}{\varepsilon} \int_{l_{3}}^{\infty}(x-y) \rho_{\text {odd }}(y) d y
$$

entonces,

$$
\varphi_{4} \equiv \psi_{6}\left(l_{4}\right)=-\frac{4 \pi}{\varepsilon} \sigma_{4} d_{2}+\frac{4 \pi}{\varepsilon}\left(\sigma_{3}+\sigma_{4}\right)\left(l_{4}-l_{3}\right)+\frac{4 \pi e}{\varepsilon} \int_{l_{1}}^{\infty}\left(l_{4}-y\right) \rho_{\text {ad }}(y) d y
$$

o también

$$
\varphi_{4}=-\frac{4 \pi}{\varepsilon} \sigma_{4} d_{2}-\frac{2 \pi}{\varepsilon}\left(\sigma_{3}+\sigma_{4}\right) a+\frac{4 \pi e}{\varepsilon} \int_{l_{1}}^{\infty}\left(l_{4}-y\right) \rho_{\alpha d}(y) d y
$$

5. Región 5: $l_{5} \equiv t_{1}+d_{1}+a / 2 \leq x \leq l_{4}$ análogamente

$$
\psi_{6}\left(l_{4}\right)=\psi_{5}\left(l_{4}\right) \equiv \psi_{4},
$$


y

$$
\varepsilon\left(\frac{d \psi_{6}(x)}{d x}\right)_{l_{4}}-\varepsilon\left(\frac{d \psi_{5}(x)}{d x}\right)_{l_{4}}=0
$$

integrando la ec.(D.2) y usando las ecs.(D.27) y (D.31),

$$
\frac{d \psi_{5}(x)}{d x}=\frac{4 \pi}{\varepsilon}\left(\sigma_{3}+\sigma_{4}\right)+\frac{4 \pi e}{\varepsilon} \int_{x}^{l_{4}} \rho_{\alpha d}(y) d y+\frac{4 \pi e}{\varepsilon} \int_{l_{1}}^{\infty} \rho_{\alpha d}(y) d y
$$

Integrando por partes la ec. (D.32) y con la ec.(D.28), tenemos que

$$
\begin{aligned}
& \psi_{5}(x)=-\frac{4 \pi}{\varepsilon}\left(\sigma_{3}+\sigma_{4}\right)\left(l_{4}+a / 2-x\right)-\frac{4 \pi \sigma_{4}}{\varepsilon} d_{2}+\frac{4 \pi \epsilon}{\varepsilon} \int_{x}^{l_{4}}(x-y) \rho_{a d}(y) d y \\
& +\frac{4 \pi e}{\varepsilon} \int_{l_{1}}^{\infty}(x-y) \rho_{a d}(y) d y
\end{aligned}
$$

$\varphi_{5}=\psi_{5}\left(l_{5}\right)=-\frac{4 \pi}{\varepsilon} \sigma_{4} d_{2}-\frac{4 \pi}{\varepsilon}\left(\sigma_{3}+\sigma_{4}\right)\left(l_{4}-l_{5}+a / 2\right)+\frac{4 \pi e}{\varepsilon} \int_{l_{1}}^{\infty}\left(l_{5}-y\right) \rho_{\alpha d}(y) d y$ $+\frac{4 \pi e}{\varepsilon} \int_{x}^{l_{5}}\left(l_{5}-y\right) \rho_{\alpha d}(y) d y$

6. Región 4: $l_{6} \equiv t_{1}+d_{1} \leq x \leq l_{5}$

aplicando nuestras condiciones de frontera

$$
\begin{gathered}
\psi_{4}\left(l_{5}\right)=\psi_{5}\left(l_{5}\right) \equiv \varphi_{5} \\
\psi_{4}\left(l_{6}\right) \equiv \varphi_{6}
\end{gathered}
$$

y

$$
\varepsilon\left(\frac{d \psi_{5}(x)}{d x}\right)_{l_{5}}-\varepsilon\left(\frac{d \psi_{4}(x)}{d x}\right)_{l_{5}}=0
$$

integrando la ec.(D.1), y aplicando las ecs.(D.33), and (D.35),

$$
\begin{aligned}
& \psi_{4}(x)=-\frac{4 \pi}{\varepsilon}\left(\sigma_{3}+\sigma_{4}\right)\left(l_{4}+\frac{a}{2}-x\right)-\frac{4 \pi \sigma_{4}}{\varepsilon} d_{2} \\
& -\frac{4 \pi \varepsilon}{\varepsilon}\left\{\int_{l_{5}}^{l_{4}}(x-y) \rho_{a d}(y) d y-\int_{l_{1}}^{\infty}(x-y) \rho_{a d}(y) d y\right\}
\end{aligned}
$$




$$
\begin{aligned}
& \varphi_{6}=-\frac{4 \pi}{\varepsilon} \sigma_{4} d_{2}+\frac{4 \pi}{\varepsilon}\left(\sigma_{3}+\sigma_{4}\right)\left(l_{6}-l_{4}-a / 2\right) \\
& +\frac{4 \pi e}{\varepsilon}\left\{\int_{l_{5}}^{l_{4}}\left(l_{6}-y\right) \rho_{\alpha d}(y) d y+\frac{4 \pi e}{\varepsilon} \int_{l_{1}}^{\infty}\left(l_{6}-y\right) \rho_{\text {add }}(y) d y\right\}
\end{aligned}
$$

7. Región 3: $l_{7} \equiv t_{1} \leq x \leq l_{6}$

de igual manera usando condiciones de frontera

$$
\begin{gathered}
\psi_{3}\left(l_{6}\right)=\psi_{4}\left(l_{6}\right) \equiv \varphi_{6} \\
\psi_{3}\left(l_{7}\right) \equiv \varphi_{7}
\end{gathered}
$$

y

$$
\varepsilon\left(\frac{d \psi_{4}(x)}{d x}\right)_{l_{6}}-\varepsilon\left(\frac{d \psi_{3}(x)}{d x}\right)_{l_{6}}=-4 \pi \sigma_{2}
$$

integrando la ec.(D.1), y usando las ecs. (D.36), y (D.38),

$$
\begin{aligned}
& \psi_{3}(x)=\frac{4 \pi}{\varepsilon}\left(\sigma_{3}+\sigma_{4}\right)\left(x-l_{4}-a / 2\right)-\frac{4 \pi \sigma_{4}}{\varepsilon} d_{2}+\frac{4 \pi \sigma_{2}}{\varepsilon}\left(x-l_{6}\right) \\
& +\frac{4 \pi e}{\varepsilon} \int_{l_{5}}^{l_{4}}(x-y) \rho_{\alpha d}(y) d y+\frac{4 \pi e}{\varepsilon} \int_{l_{1}}^{\infty}(x-y) \rho_{\alpha d}(y) d y
\end{aligned}
$$

8. Región 2: $l_{8} \equiv t_{1}-a / 2 \leq x \leq l_{7}$

continuando de la misma manera, las condiciones de frontera son

$$
\begin{gathered}
\psi_{2}\left(l_{7}\right)=\psi_{3}\left(l_{7}\right) \equiv \varphi_{7} \\
\psi_{2}\left(l_{8}\right) \equiv \varphi_{8}
\end{gathered}
$$

y

$$
\varepsilon\left(\frac{d \psi_{3}(x)}{d x}\right)_{I_{7}}-\varepsilon\left(\frac{d \psi_{2}(x)}{d x}\right)_{I_{7}}=-4 \pi \sigma_{1}
$$

integrando ec. (D.1), con las ecs. (D.39), y (D.41) tenemos,

$$
\begin{aligned}
& \psi_{2}(x)=-\frac{4 \pi}{\varepsilon}\left(\sigma_{1}+\sigma_{2}+\sigma_{3}+\sigma_{4}\right)\left(l_{7}-x\right) \\
& -\frac{4 \pi e}{\varepsilon}\left(l_{7}-x\right)\left\{\int_{l_{5}}^{l_{4}} \rho_{\alpha d}(y) d y+\int_{l_{1}}^{\infty} \rho_{a d}(y) d y\right\}+\varphi_{7}
\end{aligned}
$$


9. Región 1: $0 \leq x \leq l_{8}$

$$
\begin{aligned}
& \varphi_{7}=\frac{4 \pi}{\varepsilon}\left(\sigma_{3}+\sigma_{4}\right)\left(l_{7}-l_{4}-a / 2\right)-\frac{4 \pi \sigma_{4}}{\varepsilon} d_{2}+\frac{4 \pi \sigma_{2}}{\varepsilon}\left(l_{7}-l_{6}\right) \\
& +\frac{4 \pi e}{\varepsilon} \int_{l_{5}}^{l_{4}}\left(l_{7}-y\right) \rho_{\alpha d}(y) d y+\frac{4 \pi e}{\varepsilon} \int_{l_{1}}^{\infty}\left(l_{7}-y\right) \rho_{\alpha d}(y) d y
\end{aligned}
$$

aplicando las respectivas condiciones

$$
\begin{gathered}
\psi_{1}\left(l_{8}\right)=\psi_{2}\left(l_{8}\right) \equiv \varphi_{8} \\
\psi_{1}(0) \equiv \varphi_{d}
\end{gathered}
$$

y

$$
\varepsilon\left(\frac{d \psi_{2}(x)}{d x}\right)_{l_{8}}-\varepsilon\left(\frac{d \psi_{1}(x)}{d x}\right)_{l_{8}}=0
$$

y

$$
\varepsilon\left(\frac{d \psi_{2}(x)}{d x}\right)_{l_{8}}-\varepsilon\left(\frac{d \psi_{1}(x)}{d x}\right)_{l_{8}}=0
$$

integrando la ec.(D.2), además relacionando las ecs.(D.45) y (D.46),

$$
\begin{aligned}
& \psi_{1}(x)=-\frac{4 \pi}{\varepsilon}\left(\sigma_{1}+\sigma_{2}+\sigma_{3}+\sigma_{4}\right)\left(l_{8}-x\right) \\
& -\frac{4 \pi e}{\varepsilon}\left\{\left(\int_{l_{8}}^{l_{8}} \rho_{\alpha d}(y) d y+\int_{l_{1}}^{\infty} \rho_{\alpha d}(y) d y\right)\left(l_{8}-x\right)-\int_{x}^{l_{8}}(x-y) \rho_{\alpha d}(y) d y\right\}+\varphi_{8}
\end{aligned}
$$

con

en particular $\varphi_{d}$, es

$$
\begin{aligned}
& \varphi_{8}=\frac{4 \pi}{\varepsilon}\left(\sigma_{3}+\sigma_{4}\right)\left(l_{8}-l_{4}-\frac{a}{2}\right) \\
& -\frac{4 \pi \sigma_{4}}{\varepsilon} d_{2}-\frac{4 \pi \sigma_{2}}{\varepsilon}\left(l_{6}-l_{3}\right)-\frac{4 \pi \sigma_{1}}{\varepsilon}\left(l_{7}-l_{8}\right) \\
& +\frac{4 \pi e}{\varepsilon} \int_{l_{5}}^{l_{4}}(l-y) \rho_{\alpha d}(y) d y+\frac{4 \pi e}{\varepsilon} \int_{l_{1}}^{\infty}\left(l_{8}-y\right) \rho_{\alpha d}(y) d y
\end{aligned}
$$

$$
\begin{aligned}
& \psi_{d}=-\frac{4 \pi}{\varepsilon}\left(\sigma_{1}+\sigma_{2}+\sigma_{3}+\sigma_{4}\right) l_{8} \\
& -\frac{4 \pi}{\varepsilon}\left\{\int_{l_{5}}^{l_{4}} y \rho_{\alpha d}(y) d y+\int_{l_{2}}^{\infty} y \rho_{\alpha d}(y) d y+\int_{x}^{l_{8}} y \rho_{\alpha d}(y) d y\right\} \\
& +\frac{4 \pi}{\varepsilon}\left(\sigma_{3}+\sigma_{4}\right)\left(l_{8}-l_{4}-\frac{a}{2}\right)-\frac{4 \pi}{\varepsilon} \sigma_{2}\left(l_{6}-l_{8}\right) \\
& -\frac{4 \pi}{\varepsilon} \sigma_{1}\left(l_{7}-l_{8}\right)-\frac{4 \pi}{\varepsilon} \sigma_{4} d_{2}
\end{aligned}
$$


resumiendo los intervalos, definimos:

$$
\begin{aligned}
& l_{1}=t_{1}+d_{1}+t_{2}+d_{2}+a / 2 \\
& l_{2}=t_{1}+d_{1}+t_{2}+d_{2} \\
& l_{3}=t_{1}+d_{1}+t_{2} \\
& l_{4}=t_{1}+d_{1}+t_{2}-a / 2 \\
& l_{5}=t_{1}+d_{1}+a / 2 \\
& l_{6}=t_{1}+d_{1} \\
& l_{7}=t_{1} \\
& l_{8}=t_{1}-a / 2
\end{aligned}
$$




\section{Apéndice E}

\section{Cálculo de la Presión Osmótica}

Consideremos una placa cargada inmersa en un fluido [183] donde las dos paredes de la placa tienen una densidad de carga $\sigma_{\alpha}$ y $\sigma_{e}$ respectivamente como se observa en la figura $\mathrm{E}$ y también consideremos un elemento de fluido paralelo a la placa cargada como se observa en la misma fig. E. Las fuerzas que actúan sobre un elemento de fluido $\mathbf{F}_{1}(x)$ y $\mathbf{F}_{2}(x)$ representan las fuerzas ejercidas sobre el fluido debidas a la presión en el fluido y $q(x) \mathbf{E}$ es la fuerza eléctrica debida al campo eléctrico local E sobre la carga contenida en el elemento de fluido, $q(x)$ es la carga del elemento de fluido considerado. Como es un fluido en equilibrio la suma de todas las fuerzas es cero,

$$
\mathbf{F}_{1}+\mathbf{F}_{2}=q(x) \mathbf{E}
$$

Desglosando cada una de las fuerzas, tenemos que

$$
\begin{gathered}
\mathbf{F}_{1}=\Lambda p(x) \mathbf{i} \\
\mathbf{F}_{2}=-A p(x+d x) \mathbf{i}
\end{gathered}
$$

donde $A$ es el área del elemento de fluido, $p(x)$ y $p(x+d x)$ son las presiones en $x$ y $x+d x$, que actúan sobre el elemento de fluido, el vector unitario i está en la dirección $x$ en que se aplican las fuerzas, la carga de un elemente infinitesimal lo escribimos como

$$
d q=-A d x \rho_{t^{\prime}}(x)
$$

y el campo eléctrico es

$$
\mathbf{E}=-\left(\frac{\partial u(x)}{\partial x}\right) \mathbf{i}
$$

donde $\psi(x)$ es el potencial eléctrico en esa posición, sustituyendo làs ecuaciones anteriores en la suma de fuerzas, tenemos

$$
p(x)-p(x+d x)=\rho_{c i}(x) \frac{\partial \psi(x)}{\partial x} d x
$$




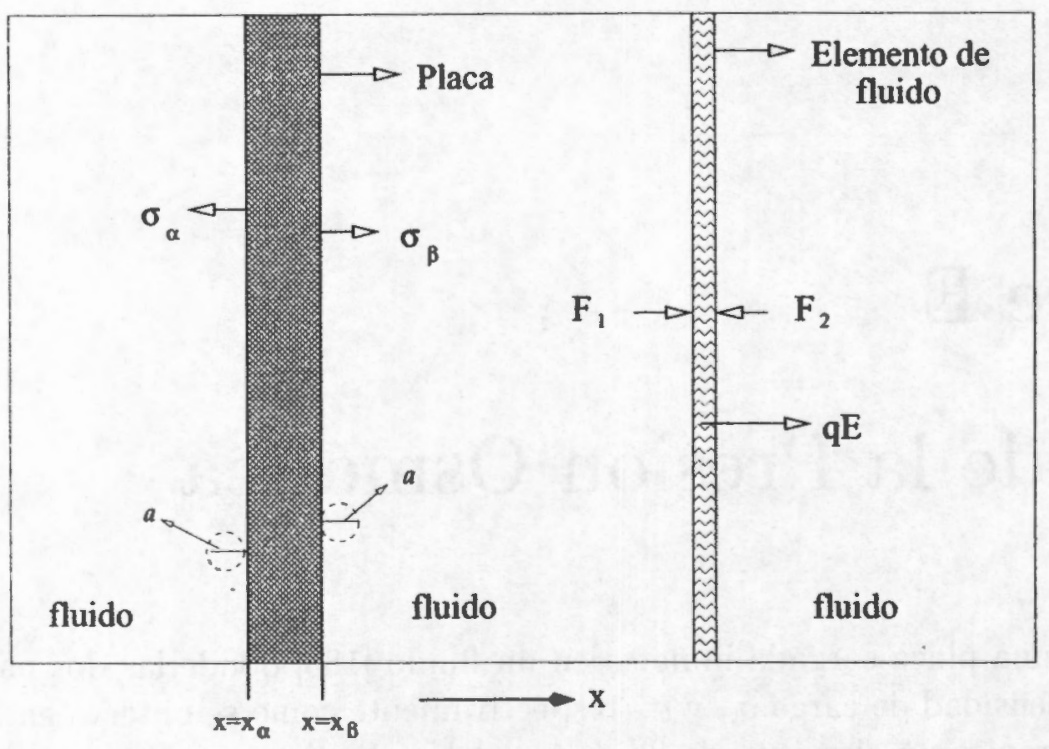

Figura E.1: representación esquemática del fluido

Haciendo un desarrollo en serie a primer orden para $p(x+d x)$,

$$
p(x+d x)=p(x)+\left(\frac{d p}{d x}\right) d x+\ldots
$$

aplicando la ecuación de Poisson,

$$
\rho_{e l}(x)=-\frac{\epsilon}{4 \pi}\left(\frac{d^{2} \psi(x)}{d x^{2}}\right)
$$

sustituyendo en la ecuación (E.1), obtenemos

$$
\frac{d p}{d x}=\frac{\epsilon}{4 \pi}\left(\frac{d^{2} \psi(x)}{d x^{2}}\right) \frac{d \psi(x)}{d x}
$$

integrando esta ecuación entre $\infty$ y $x$ se tiene

$$
p(x)-p(\infty)=\frac{\epsilon}{4 \pi} \int_{\infty}^{x} \frac{d^{2} \psi(x)}{d x^{2}} \frac{d \psi(x)}{d x} d x
$$

integrando por partes;

$$
p(x)-p(\infty)=\frac{\epsilon}{8 \pi}\left(\frac{d \psi(x)}{d x}\right)^{2}
$$

Si definimos $p(\infty) \equiv \Pi_{b}$, como la presión del bulto, entonces

$$
p(x)=\frac{\epsilon}{8 \pi} E(x)^{2}+\Pi_{b}
$$


La presión en la pared debe ser igual al intercambio de ímpetu por unidad de área y por unidad de tiempo por estar en contacto con el fiuido, entonces en el contacto con la pared del lado derecho

$$
p_{\beta}\left(x=x_{\beta}\right)=k T \sum_{i=i}^{N} p_{i}\left(x=x_{\beta}+a / 2\right)
$$

0

$$
k T \sum_{i=1}^{N} \rho_{i}\left(x=x_{\beta}+a / 2\right)=\frac{\epsilon}{8 \pi} E^{2}\left(x_{\beta}\right)+\Pi_{\beta}
$$

de manera similar para el lado izquierdo de la placa

$$
p_{\alpha}\left(x=x_{\alpha}\right)=k T \sum_{i=1}^{N} \rho_{i}\left(x=x_{\alpha}-a / 2\right)
$$

0

$$
k T \sum_{i=1}^{N} \rho_{i}\left(x=x_{\alpha}-a / 2\right)=\frac{c}{8 \pi} E^{2}\left(x_{\alpha}\right)+\Pi_{\alpha}
$$

donde $x_{\alpha}$ y $x_{\beta}$ son los puntos de contacto con la placa del lado izquierdo y del lado derecho respectivamente, $a / 2$ es el radio de los iones ien este caso suponemos para simplificar un poco que todos tienen el mismo radio), $p_{a} \searrow p_{\beta}$ son las presiones del lado izquierdo $y$ derecho respectivamente y $\Pi_{\alpha}$ y $\Pi_{\beta}$ son las presicnes de bulto del lado izquierdo y derecho respectivamente.

Por otro lado, para encontrar el campo eléctrico sobre una superficie de la placa o en el punto de contacto, tenemos

$$
E\left(x_{0}\right)=\frac{4 \pi}{\epsilon} \sigma_{m}
$$

donde $\sigma_{m}$ es la densidad de carga inducida por la pared, en el fluido, definida anteriormente en las ecuaciones (1.67) y (1.68), esta ecuación puede deducirse a partir del potencial electrostático (vease apéndice D).

Además, puede observarse que la densidad de carga se expresa en función de la posición de contacto en la pared más el radio del ión para el lado derecho, y menos el radio del ión para la parte izquierda, esto es por la razón de que en este modelo los centros de los iones no pueden tocar los puntos sobre la superficie, y hemos liamado a esta posición como punto de máximo acercamiento, $E\left(x_{0}\right)$ es el campo para $x=x_{0}$ que es el punto de contacto con una pared de la placa cargada. Entonces sustituyencio en las ecuaciones (E.2), (E.3) la ec. (E.4) y definiendo

$$
\sum_{i=1}^{N} \rho_{i}\left(x=x_{\beta}+a / 2\right) \equiv \rho_{\beta}\left(x_{\beta}+a / 2\right)
$$

para el lado derecho de la placa $y$ 


$$
\sum_{i=1}^{N} \rho_{i}\left(x=x_{\alpha}-a / 2\right) \equiv \rho_{a}\left(x_{\alpha}-a / 2\right)
$$

para el lado izquierdo de la placa, obtenemos

$$
\begin{aligned}
& k T \rho_{\alpha}\left(x_{\alpha}-a / 2\right)=\frac{2 \pi}{\epsilon} \sigma_{\alpha}^{2}+\Pi_{\alpha} \\
& k T \rho_{\beta}\left(x_{\beta}+a / 2\right)=\frac{2 \pi}{\epsilon} \sigma_{\beta}^{2}+\Pi_{\beta}
\end{aligned}
$$

donde $\sigma_{\alpha}$ y $\sigma_{\beta}$ son las densidades de carga superficial en las paredes izquierda y derecha de la placa respectivamente. De esta manera la presión Osmótica la podemos escribir así

$$
p=\Pi_{\beta}-\Pi_{\alpha}=k T\left[\rho_{\beta}\left(x_{\beta}+a / 2\right)-\rho_{\alpha}\left(x_{\alpha}-a / 2\right)\right]-\frac{2 \pi}{\epsilon}\left(\sigma_{\beta}^{2}-\sigma_{\alpha}^{2}\right)
$$




\section{Apéndice $\mathrm{F}$}

\section{Método Numérico.}

La ecuación integral de Orstein-Zernike, requiere de métodos numéricos computacionales para su solución pues no se pueden obtener resultados analíticos en nuestro caso. Las expresiones para $g(x)$ son resueltas, en nuestro caso usando el método de elemento finito $[184,185,186]$, donde el dominio se divide en un conjunto de subdominios o "elementos" elegidos convenientemente.

El método de elemento finito (MEF) se utiliza para hallar la solución de las ecuaciones integrales. El procedimiento consiste en definir una función $f(x)$ sobre un intervalo $a \leq x \leq b$, la cual satisface una ecuación integral de la siguiente forma:

$$
f(x)=\exp \left\{M(x)+\int_{a}^{b} f(y) c(x, y) d y\right\}-1
$$

La idea principal es que las funciones pueden ser representadas en términos de funciones base definidas por pedazos sobre subregiones del dominio llamados elementos finitos. Se propone dividir el dominio de la función en $\mathrm{N}$ intervalos finitos y se hace un desarrollo de la función en una base conveniente

$$
f(x)=\sum_{k=1}^{N} w_{k} \phi_{k}(x)
$$

donde $a \leq x \leq b, y$ el conjunto $\left\{\phi_{1}(x), \phi_{2}(x), \ldots, \phi_{N}(x)\right\}$ es una base definida sobre el intervalo de la función $f(x)$ y $\left\{w_{1}, w_{2}, \ldots, w_{N}\right\}$ son los coeficientes del desarrollo de la función en su dominio. Sustituyendo la Ec. F.2 en la Ec. F.1 se obtiene

$$
\sum_{k=1}^{N} w_{k} \phi_{k}(x)=\exp \left\{M(x)+\int_{a}^{b} \sum_{k=1}^{N} w_{k} \phi_{k}(y) c(x \cdot y) d y\right\}-1
$$

Definiendo

$$
c_{k}(x)=\int_{a}^{b} \phi_{k}(y) c(x, y) d y
$$

la Ec. F.3 se puede escribir como

$$
\sum_{k=1}^{N} w_{k} \phi_{k}(x)=\exp \left\{M(x)+\sum_{k=1}^{N} u_{k} c_{k}(x)\right\}-1
$$


A continuación se genera un sistema de ecuaciones no-lineales que es resuelto por un algoritmo de Newton-Rapson para sistemas no-lineales reduciendose a un sistema lineal que se resuelve por otro algoritmo, por el método de eliminación Gaussiana, resumido enseguida.

En cada uno de estos subdominios la función base toma una forma sencilla como son polinomios de segundo grado en nuestro caso, el arreglo de la subregiones se llama malla. Para construir las funciones base primero se divide el dominio de la función en cierto número finito de elementos de longitud arbitraria. A los puntos de la partición se les denomina nodos. Al conjunto de elementos y puntos nodales que constituyen el dominio del problema aproximado se les conoce como malla del elemento finito. Los criterios para generar las funciones base son: a) Son funciones sencillas definidas por pedazos de la malla. b) Los coeficientes $w_{i}$ (que definen la solución aproximada de $f(x)$ ), coinciden con los valores de la solución exacta en los nodos de la malla.

En este trabajo se han seleccionado como funciones base polinomios cuadráticos para cada elemento y se ha definido un punto auxiliar dentro del mismo elemento. Se define la coordenada local para cada elemento finito como

$$
\xi=\left(2 x-x_{1}-x_{D}\right) /\left(x_{1}-x_{D}\right)
$$

con $-1 \leq \xi \leq 1$, donde $x_{1}$ y $x_{D}$ representan posiciones de los nodos izquierdo y derecho respectivamente que delimitan al elemento en cuestión. Las funciones base para cada elemento de la malla se seleccionan de la siguiente manera:

$$
\begin{aligned}
& \phi_{1}=(1 / 2) \xi(\xi-1) \\
& \phi_{2}=1-\xi^{2} \\
& \phi_{3}=(1 / 2) \xi(\xi+1)
\end{aligned}
$$

La versión de colocación del método propone que las funciones de peso en la ecuación F.5, sean exactamente el valor de la función en dicho punto, tal que al evaluar $\phi_{1}, \phi_{2}, \phi_{3}$, en tal punto se obtiene

$$
w_{k}=\exp \left\{M\left(x_{k}\right)+\sum_{n=1}^{N} w_{k} c_{n}\left(x_{k}\right)\right\}-1
$$

o también

$$
w_{k}=\exp \left\{M_{k}+\sum_{n=1}^{N} w_{n} c_{k n}\right\}-1
$$

donde se ha definido $c_{n}\left(x_{k}\right)=c_{k n}$. Como el valor en el nodo de las funciones base evaluadas en el nodo $x_{k}$ está dado por

$$
\phi_{n}\left(x_{k}\right)=\delta_{n k}
$$

donde $\delta_{n k}$ es la delta de Kroneker. Asi se reduce el problema de una ecuación integral a un sistema de ecuaciones algebraicas no lineales:

$$
\begin{aligned}
& f_{1}\left(w_{1}, w_{2}, \ldots, w_{N}\right)=u_{1}-\operatorname{Exp}\left\{M_{1}+c_{11} u_{1}+c_{12} u_{2}, \ldots, c_{1 N} u_{N}\right\}+1=0 \\
& f_{2}\left(u_{1}, w_{2}, \ldots, w_{N}\right)=w_{2}-\operatorname{Exp}\left\{M_{2}+c_{21} w_{1}+c_{22} w_{2}, \ldots, c_{2 N} w_{N}\right\}+1=0 \\
& f_{N}\left(u_{1}, w_{2} \ldots, w_{N}\right)=w_{N}-\operatorname{Exp}\left\{M_{N}+c_{N 1} w_{1}+c_{N 2} w_{2}, \ldots, c_{N N} w_{N}\right\}+1=0
\end{aligned}
$$


La solución de este sistema de ecuaciones no lineales se realiza aplicando el método iterativo de Newton, escritas como:

$$
w_{k}^{(n+1)}=w_{k}^{(n)}+\sum_{i=1}^{N} A_{k i} f_{i}\left(w_{1}^{(n)}, w_{2}^{(n)}, \ldots, w_{N}^{(n)}\right)
$$

donde $A_{k i}$ son las componentes de la matriz inversa del Jacobiano y están dadas por $J_{i j}=$ $\partial f_{i}\left(w_{1}^{(n)}, w_{2}^{(n)}, \ldots, w_{N}^{(n)}\right) / \partial w_{j}^{(n)}$

$$
J_{i j}=\delta_{i j}-c_{i j} \exp \left\{M_{i}+\sum_{n=1}^{N} c_{i n} w_{n}\right\}
$$

con

$$
A_{i j}=\left[\mathbf{J}^{-1}\right]_{i j}
$$


Apéndice G

Gráficas 

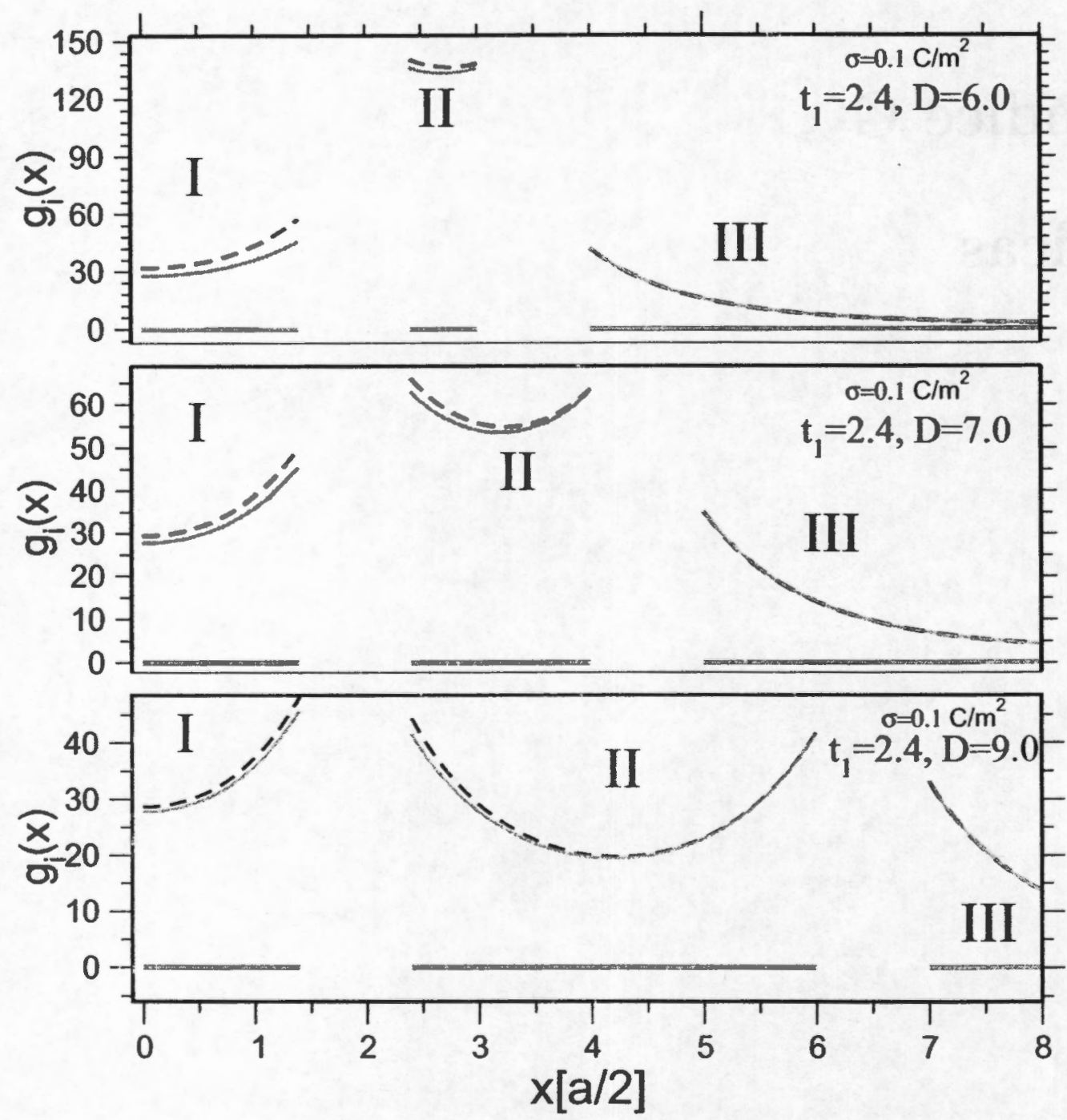

Figura G.1: Perfiles de concentración de contraiones y coiones $\left(g_{-}(x)\right.$ y $g_{+}(x)$ respectivamente, $i=-,+$ ) como función de la distancia perpendicular a las placas $x$, para un electrolito $1: 1$ y $0.1 \mathrm{M}$, confinado entre cuatro placas cargadas con $\sigma_{1}=0.1 \mathrm{C} / \mathrm{m}^{2}=\sigma_{2}=$ $\sigma_{3}=\sigma_{4}$, para las separaciones de placas siguientes $t_{1}$ y $D$ como se indican en la figura. La distancia $x$ es desde el centro del sistema, medido en unidades de [a/2]. Las líneas punteadas y continuas se refieren a los contraiones y coiones respectivamente. Las líneas oscuras se refieren a las cuatro placas y las líneas claras a dos placas, el ancho de las placas no se representa en las gráficas. 


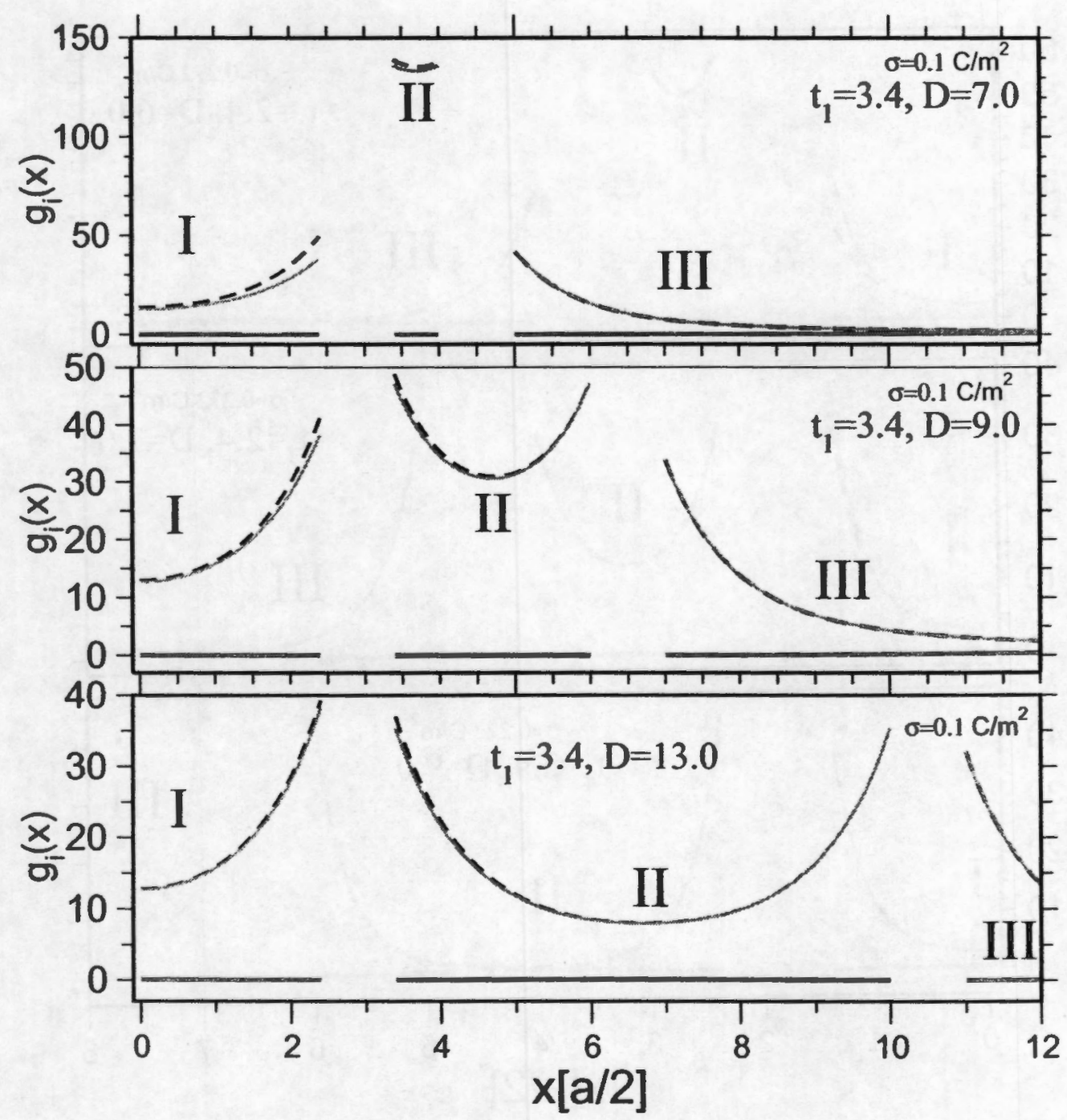

Figura G.2: Perfiles de concentración de contraiones y coiones como función de la distancia perpendicular a las placas $x$, con las mismas características del fluido que en la figura anterior, para las separaciones de placas indicadas en la figura. Las líneas tienen la misma notación que en la figura 3.18 

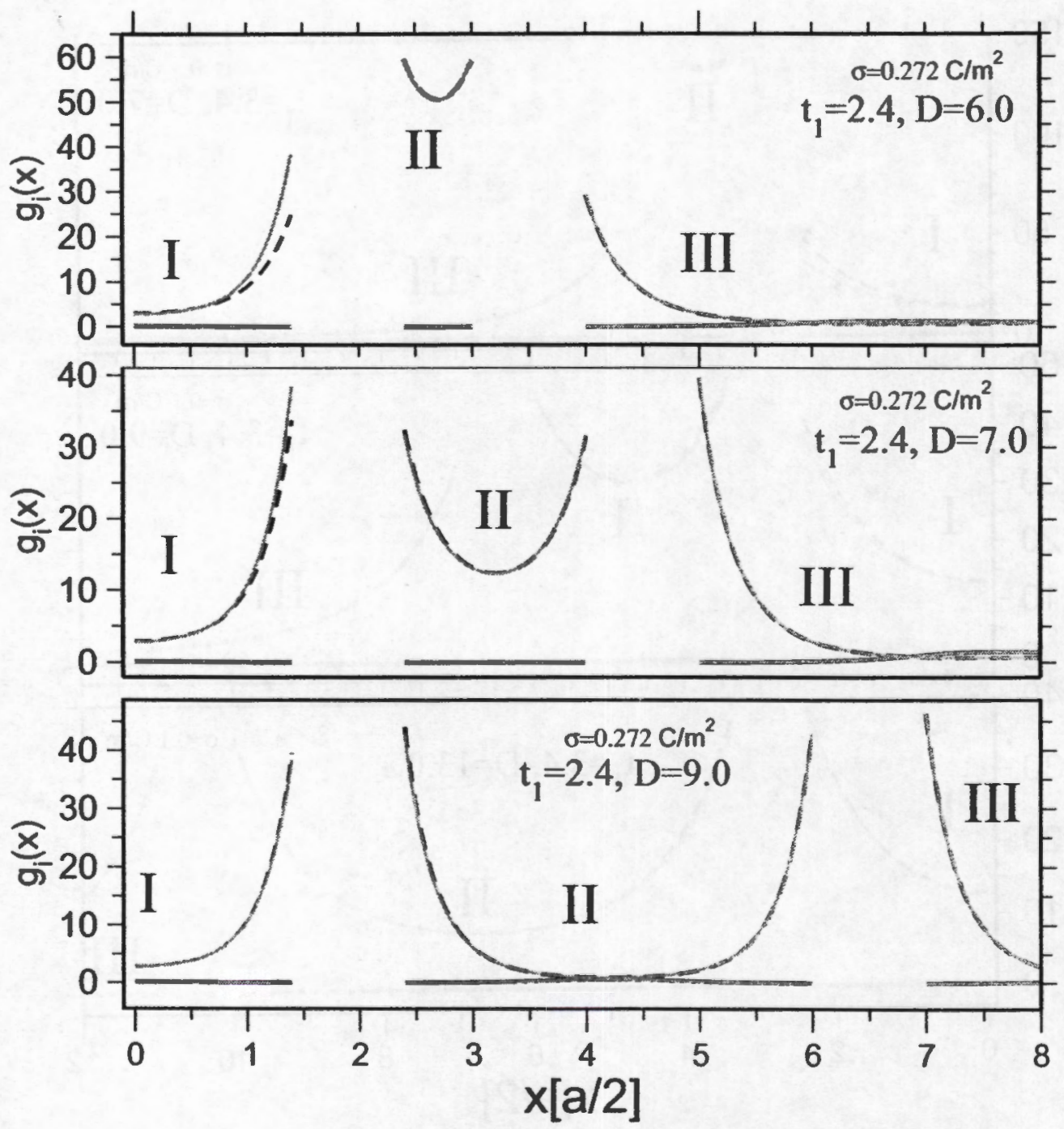

Figura G.3: Perfiles de concentración de contraiones y coiones como función de la distancia perpendicular a las placas $x$, para un electrolito $2: 2$ y $0.5 \mathrm{M}$, confinado entre cuatro placas cargadas con $\sigma_{1}=0.272 \mathrm{C} / \mathrm{m}^{2}=\sigma_{2}=\sigma_{3}=\sigma_{4}$, para las separaciones de placas indicadas en la figura. Las líneas tienen la misma notación que en la figura 3.18. 

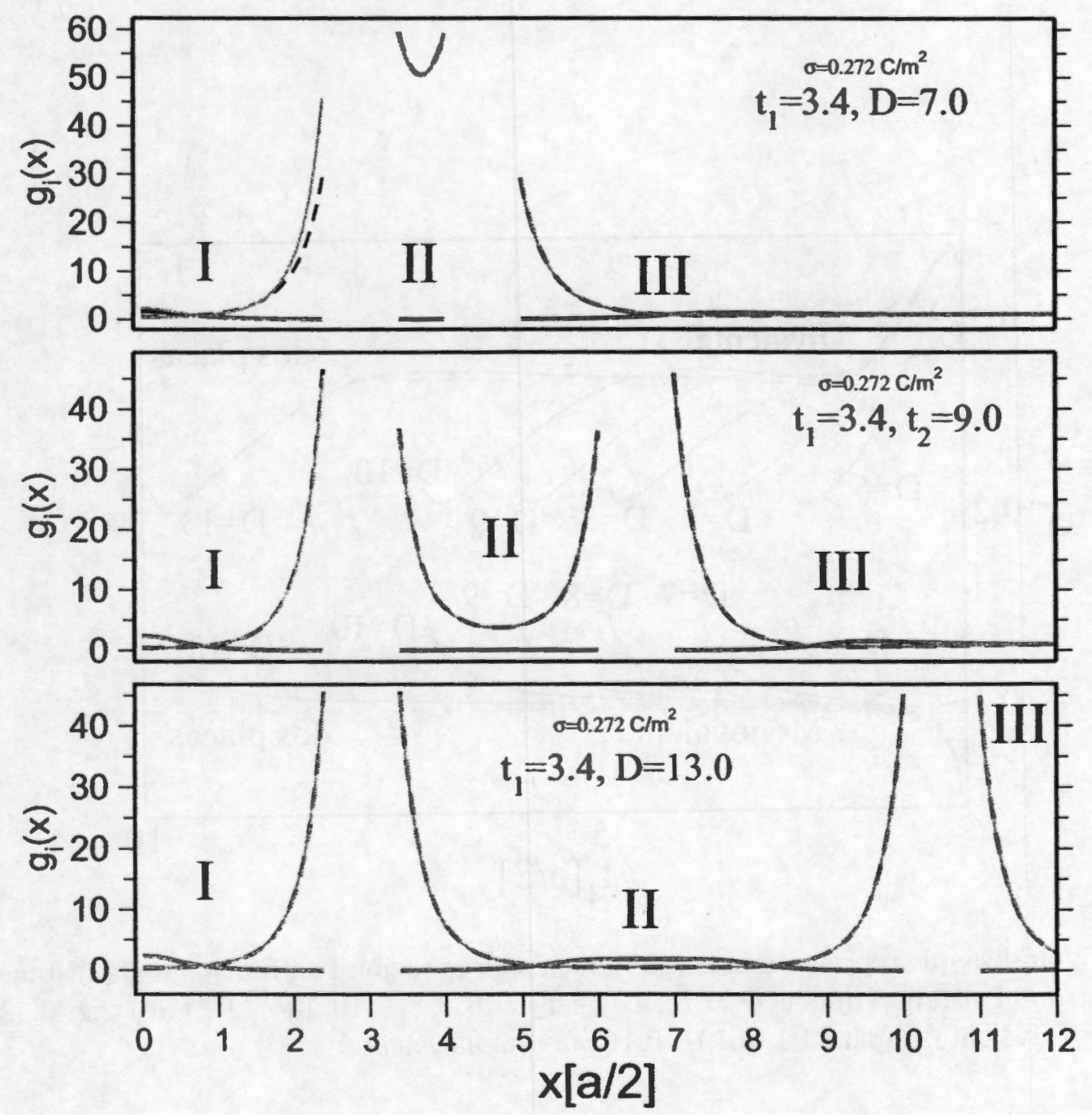

Figura G.4: Perfiles de concentración de contraiones y coiones como función de la distancia perpendicular a las placas $x$, para un electrolito $2: 2$ y $0.5 \mathrm{M}, \sigma_{1}=0.272 C / \mathrm{m}^{2}=\sigma_{2}=\sigma_{3}=\sigma_{4}$, para las separaciones de placas indicadas en la figura. Las líneas tienen la misma notación que en la figura 3.18 . 


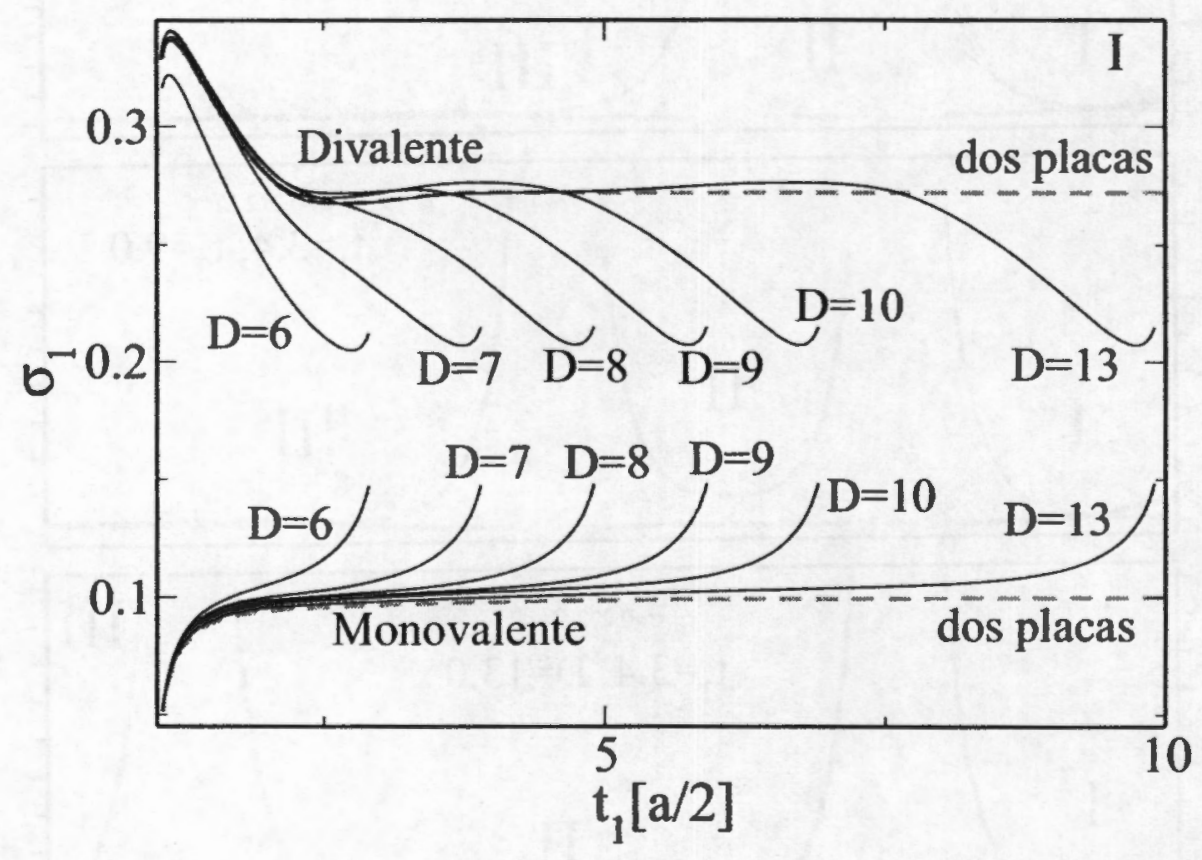

Figura G.5: Gráfica que representa la carga inducida en la región I en función de la distancia $t_{1}$, del centro del sistema, donde $D=t_{1}+d_{1}+t_{2}=6,7,8,9,10,13(a / 2)$. Para 2:2, $0.5 M$, $0.272 \mathrm{C} / \mathrm{m}^{2}$ (divalente) y para $1: 1,0.1 \mathrm{M}, 0.1 \mathrm{C} / \mathrm{m}^{2}$ (monovalente). 


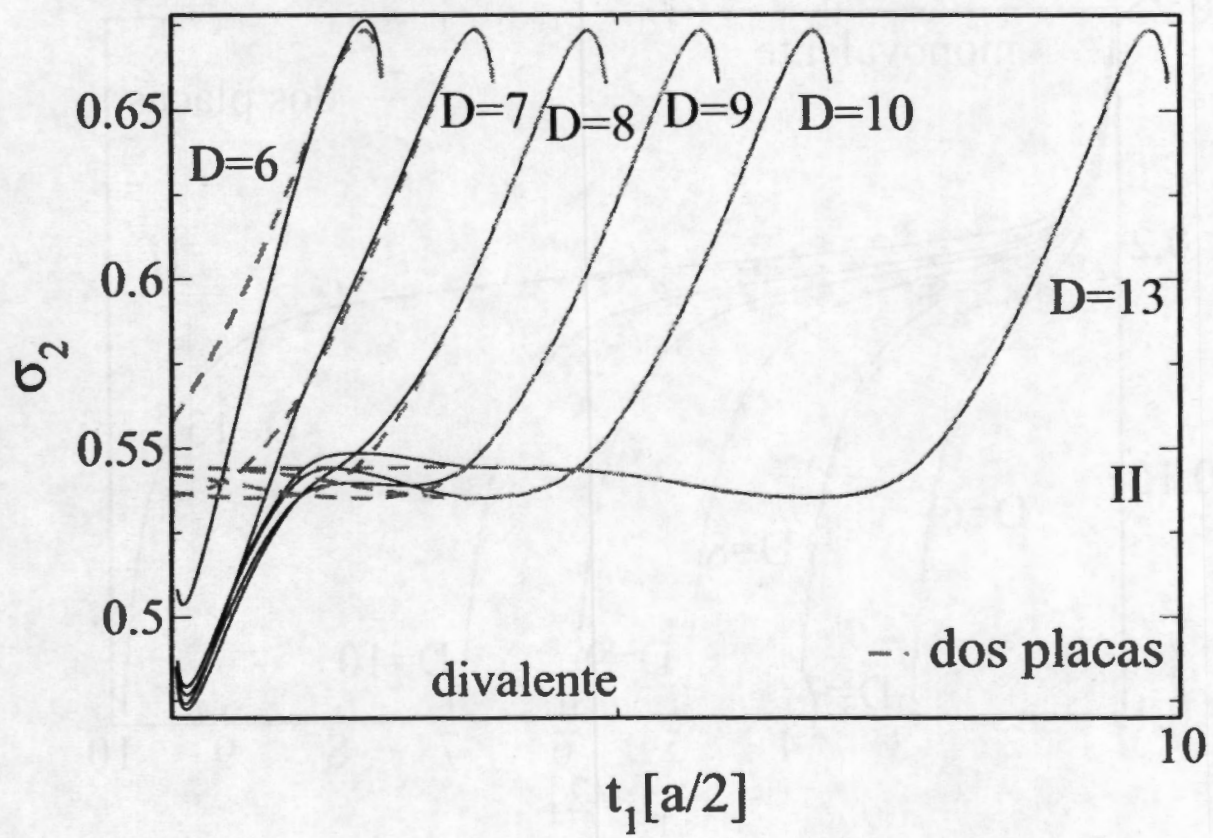

Figura G.6: Gráfica que representa la carga inducida en la región II en función de la distancia $t_{1}$, para diferentes valores de $D$, que están señalados en la figura. Las líneas obscuras corresponden a las cuatro placas. 


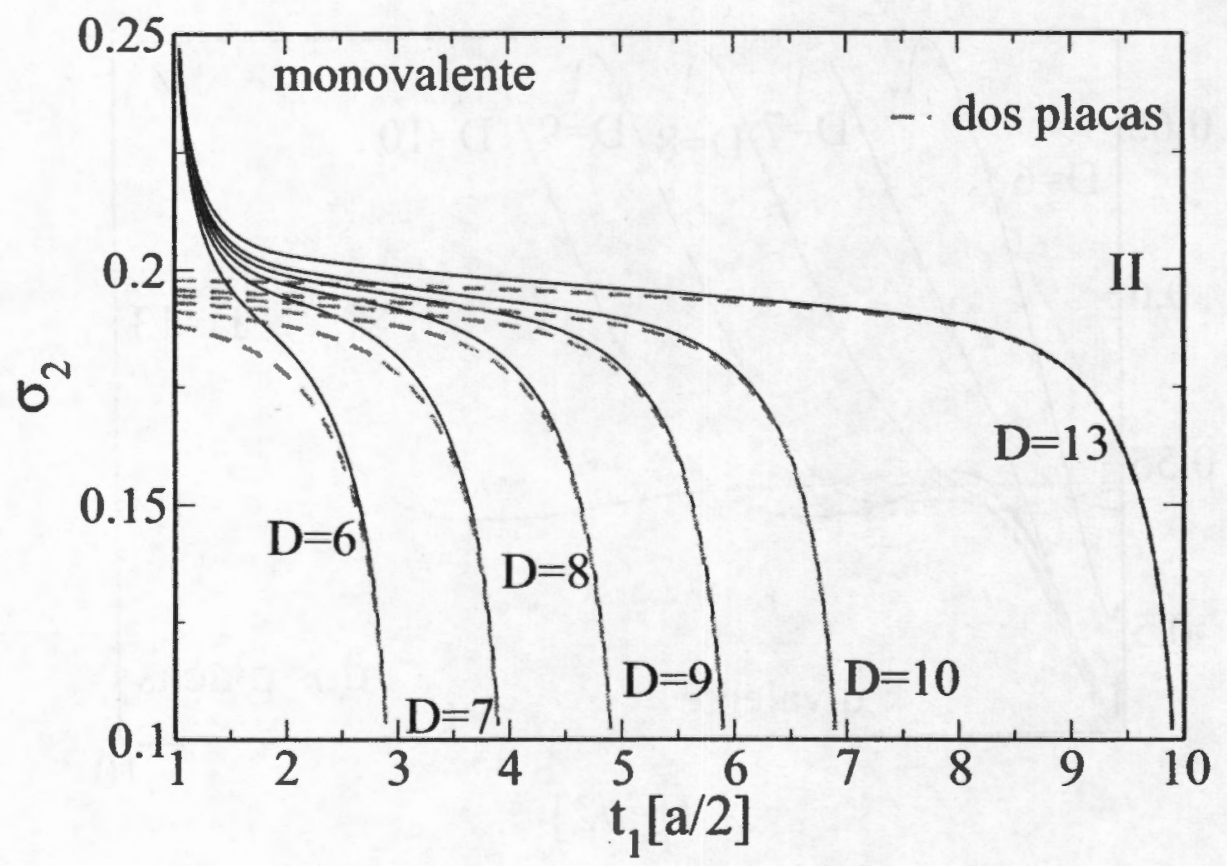

Figura G.7: Gráfica que representa la carga inducida en la región II en función de la distancia $t_{1}$, para diferentes valores de $D$, que están señalados en la figura. Las líneas obscuras corresponden a las cuatro placas. 


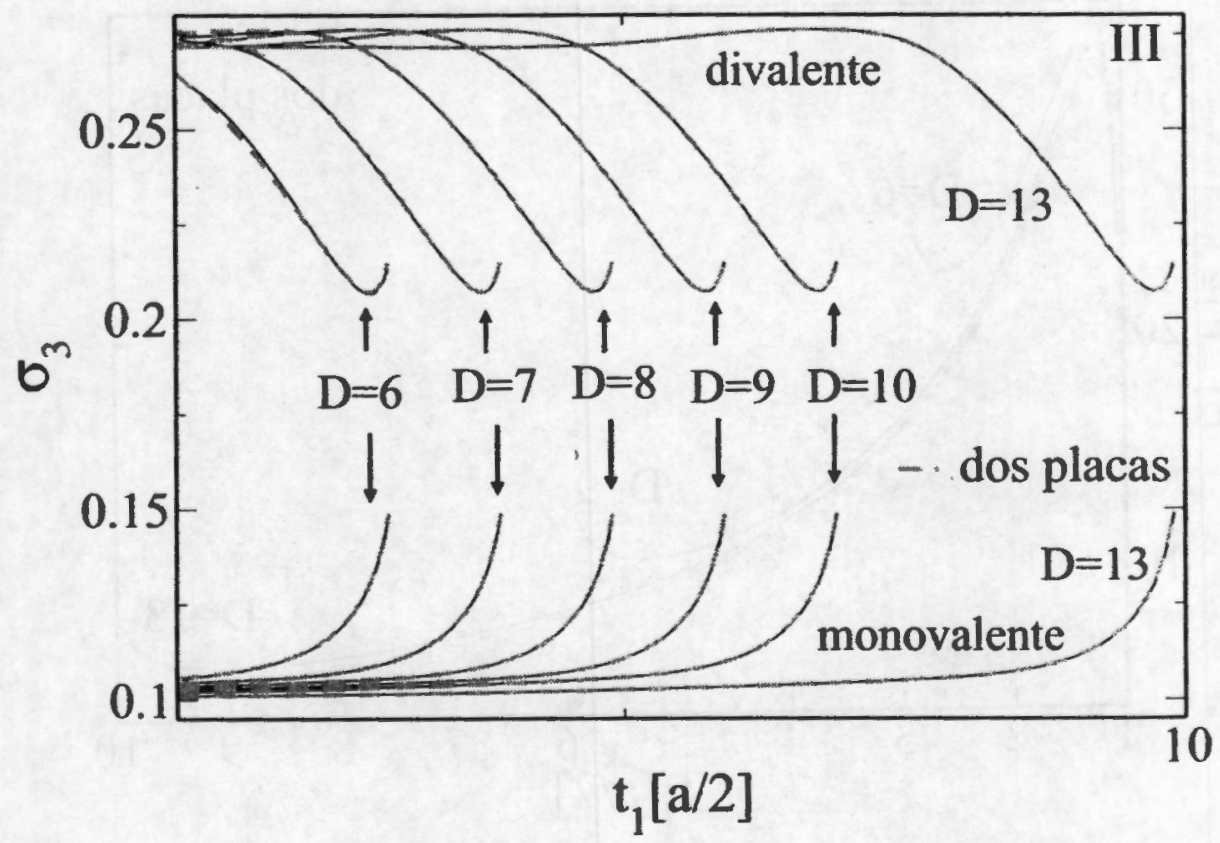

Figura G.8: Gráfica que representa la carga inducida en la región III en función de la distancia $t_{1}$, para los casos monovalente y divalente indicados en la figura, para diferentes valores de $D$, que están señalados en la figura. Las líneas obscuras corresponden a las cuatro placas. 


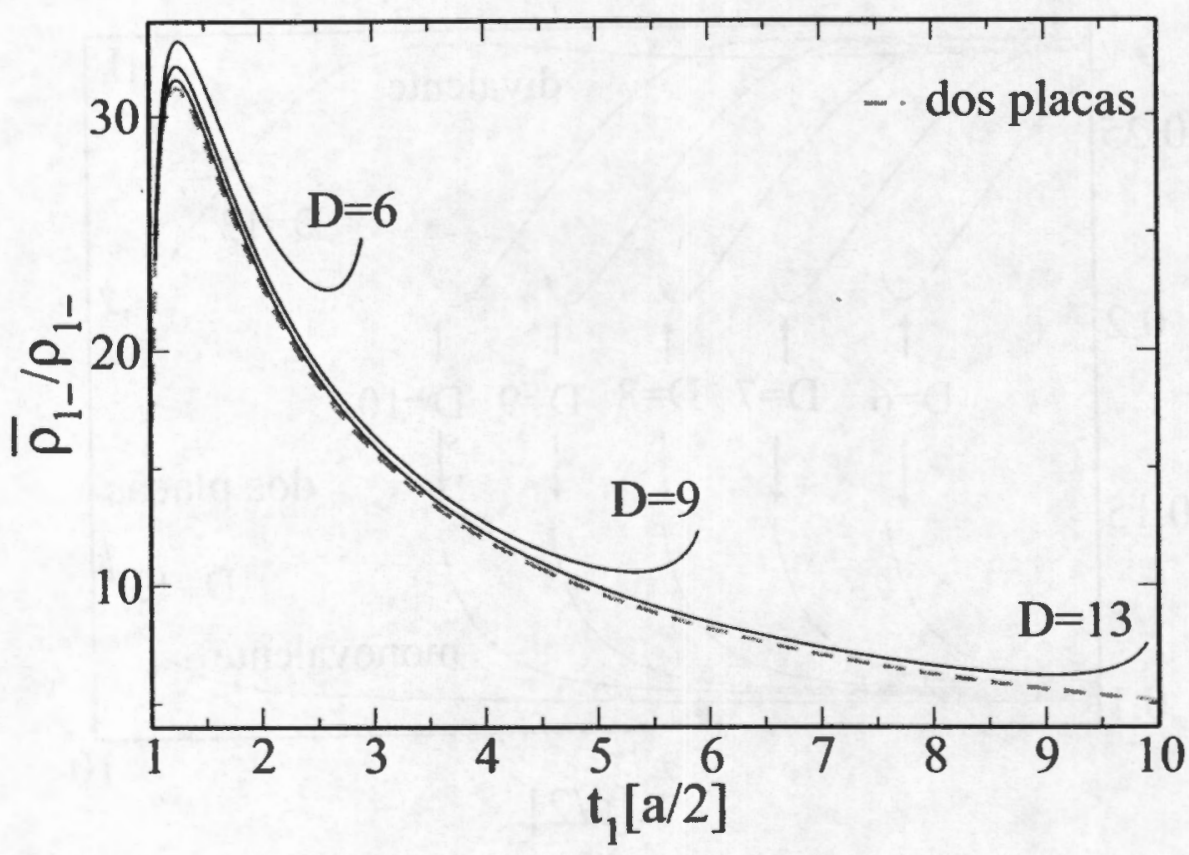

Figura G.9: Gráfica que representa la densidad de carga promedio negativa o concentración promedio de contraiones adsorvida $\left(\bar{\rho}_{1-} / \rho_{1-}\right)$, como función de la distancia $t_{1}$ para la región I, $D=t_{1}+d_{1}+t_{2}=6,7,8,9,10,13(a / 2)$, para el caso, monovalente, como en la figura 3.13. 


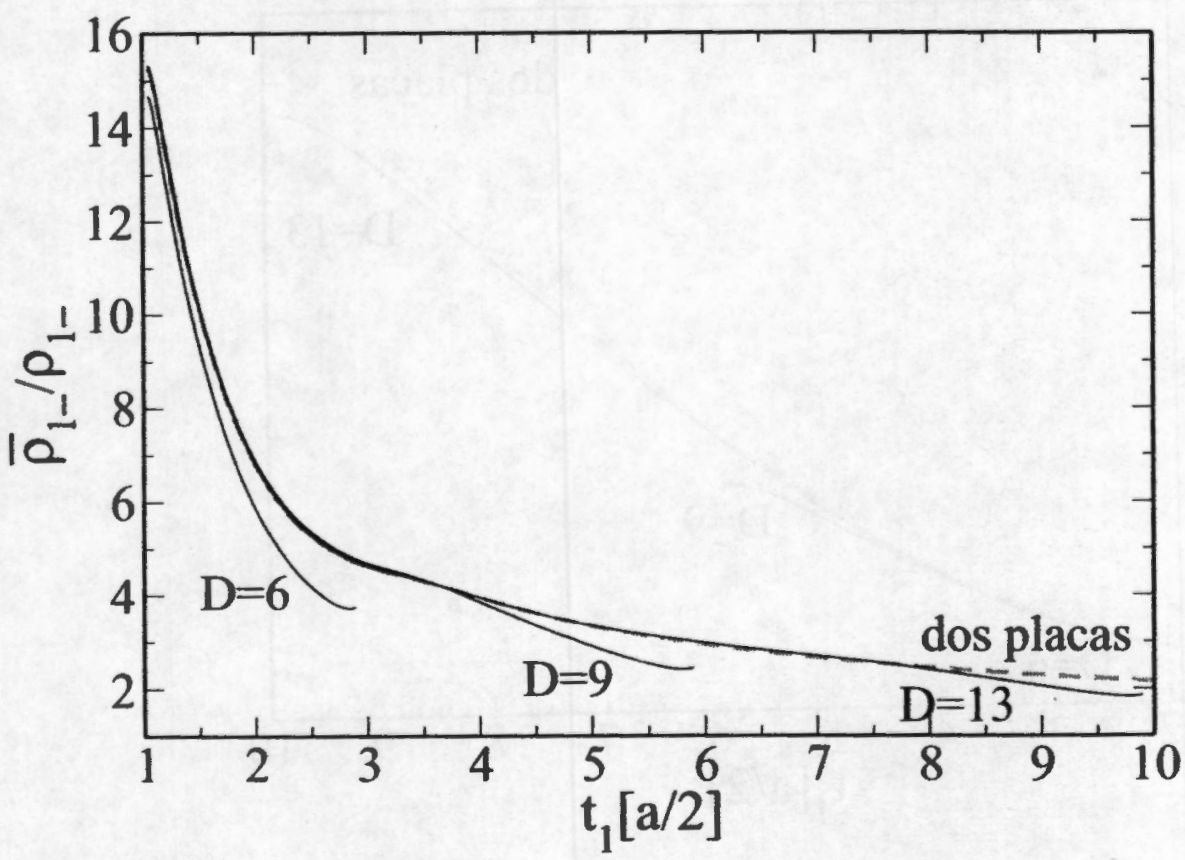

Figura G.10: Gráfica que representa la densidad de carga promedio negativa o concentración promedio de contraiones adsorvida $\left(\bar{\rho}_{1-} / \rho_{1-}\right)$, como función de la distancia $t_{1}$ para la región 1, $D=t_{1}+d_{1}+t_{2}=6,7,8,9,10,13(a / 2)$, para el caso divalente, como en la figura 3.13. 


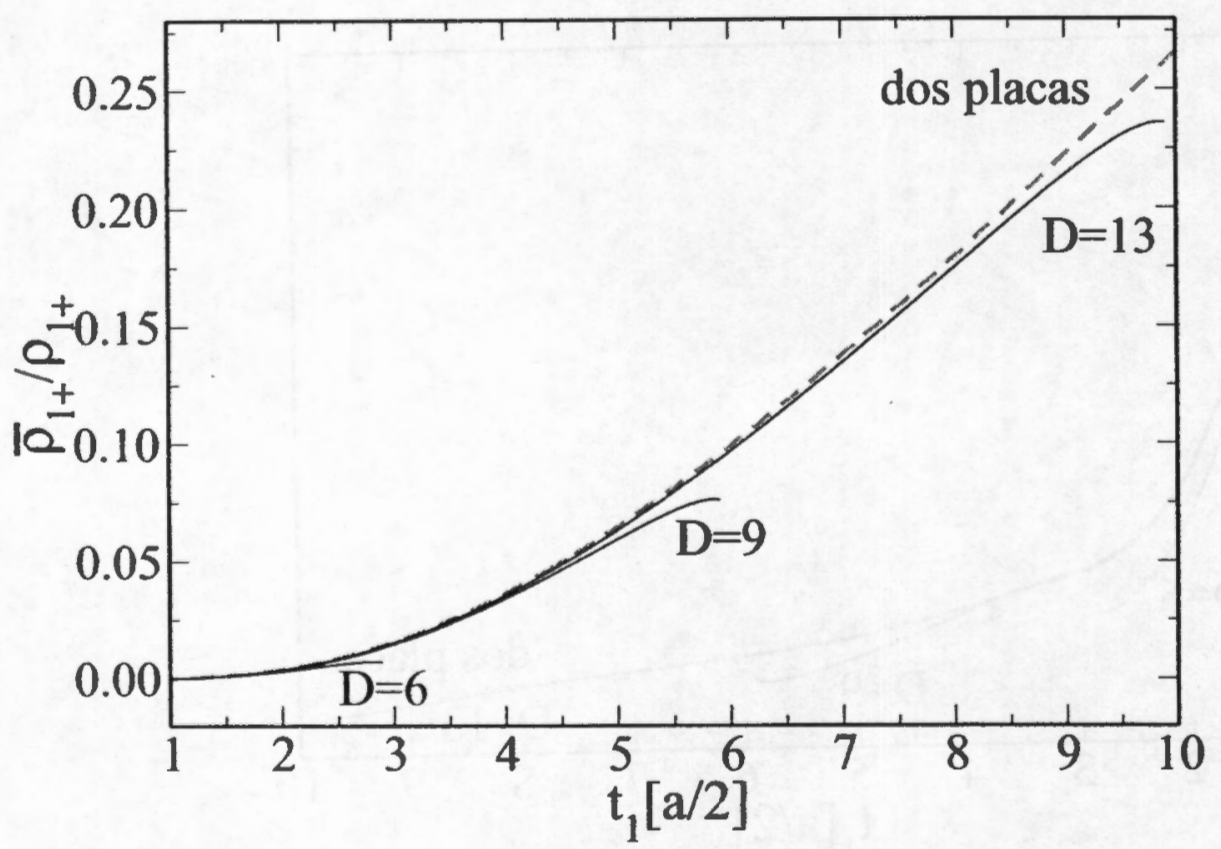

Figura G.11: Gráfica que representa la densidad de carga reducido promedio positiva o concentración promedio de carga positiva reducido (coiones $\bar{\rho}_{1+} / \rho_{1+}$ ) adsorvida para el caso monovalente, como función de la distancia $t_{1}$ para la región $I, y$ con distancias fijas $D=$ $t_{1}+d_{1}+t_{2}=6,9,13(a / 2)$, como en la figura 3.13 . 


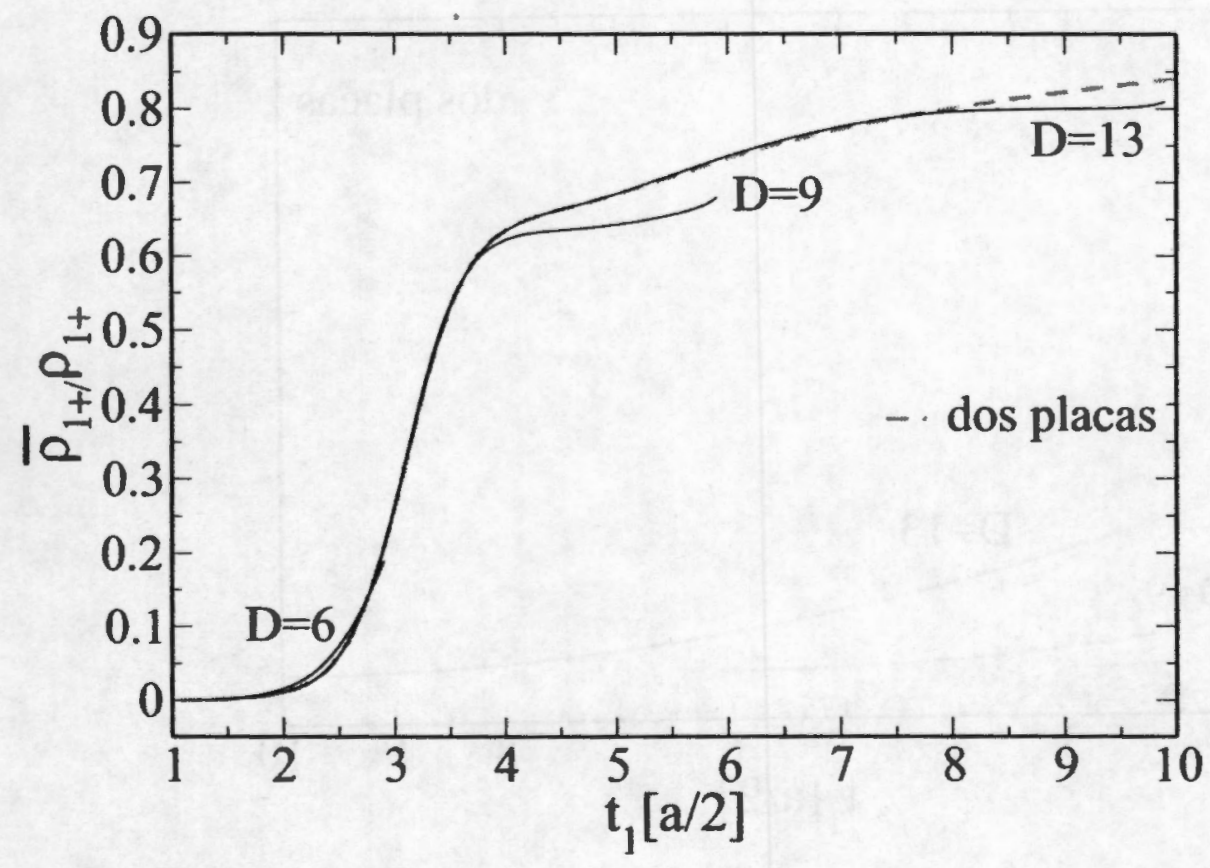

Figura G.12: Gráfica que representa la densidad de carga positiva reducida o concentración promedio de carga positiva (coiones $\bar{\rho}_{1+} / \rho_{1+}$ ) adsorvida para el caso divalente, como función de la distancia $t_{1}$ para la región $I, y$ con distancias fijas, $D=t_{1}+d_{1}+t_{2}=6,9,13(a / 2)$, para los como en la figura 3.13. 


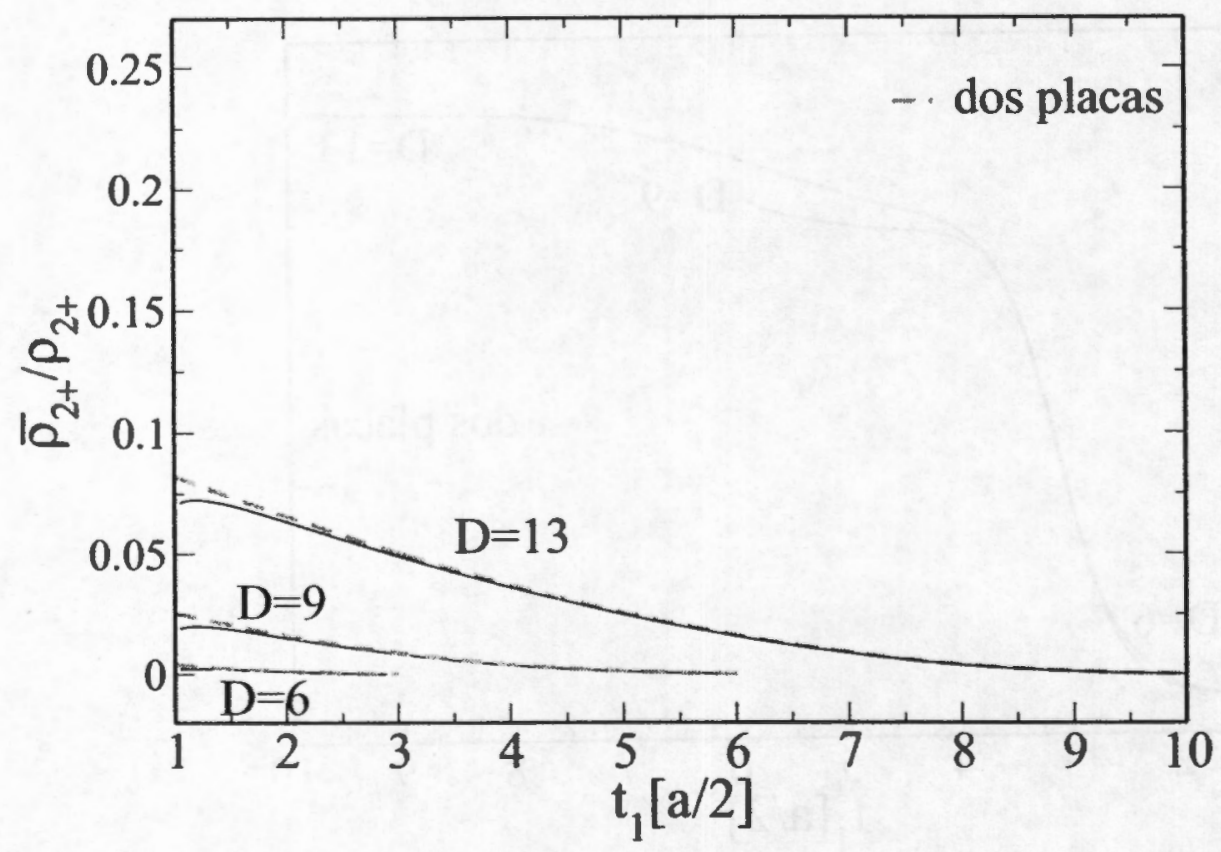

Figura G.13: Gráfica que representa la concentración promedio reducido de coiones $\left(\bar{\rho}_{2+} / \rho_{2+}\right)$ como función de $t_{1}$ en la región II para las distancias fijas $D=t_{1}+d_{1}+t_{2}=6,9,13(a / 2)$. Para el caso monovalente como en la figura 3.13. 


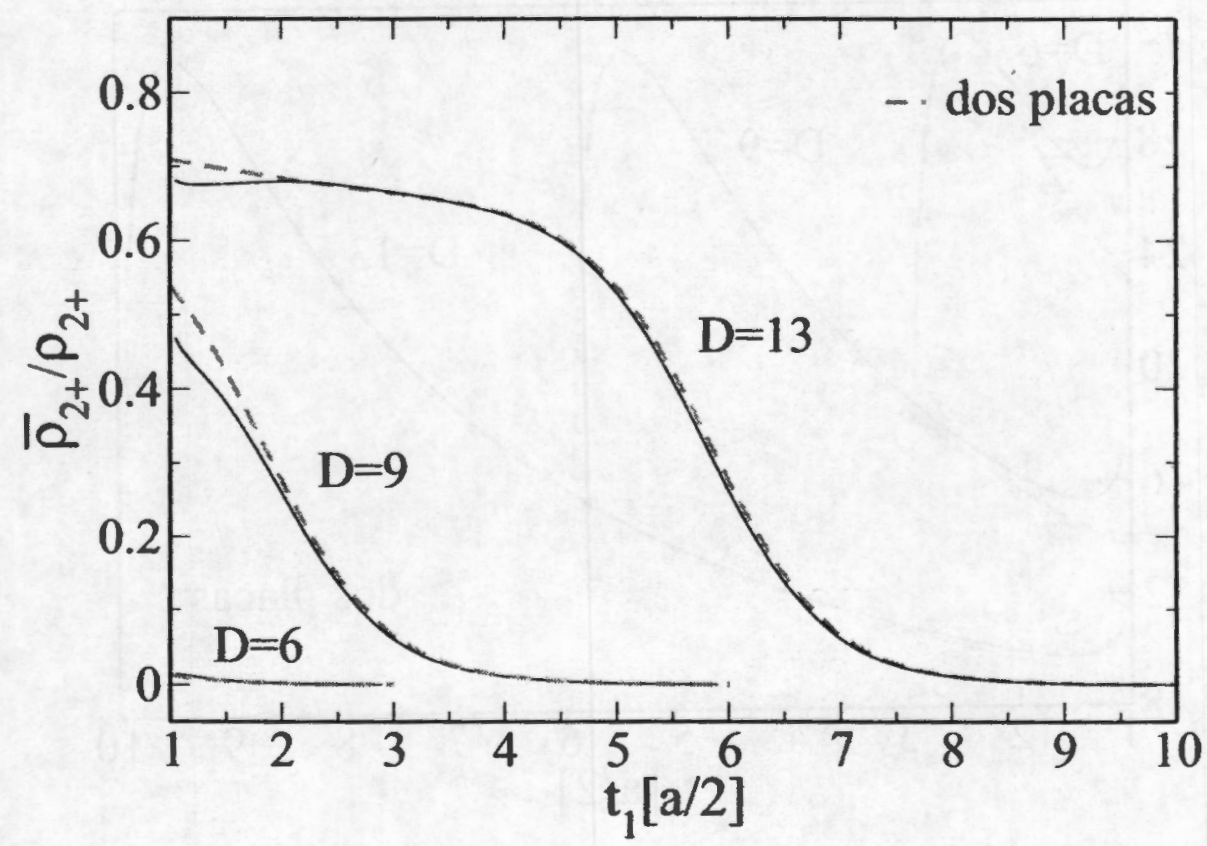

Figura G.14: Gráfica que representa la concentración promedio reducido de coiones $\left(\bar{\rho}_{2+} / \rho_{2+}\right)$ como función de $t_{1}$ en la región II para las distancias fijas $D=t_{1}+d_{1}+t_{2}=6,9,13(a / 2)$. Para el caso divalente como en la figura 3.13. 


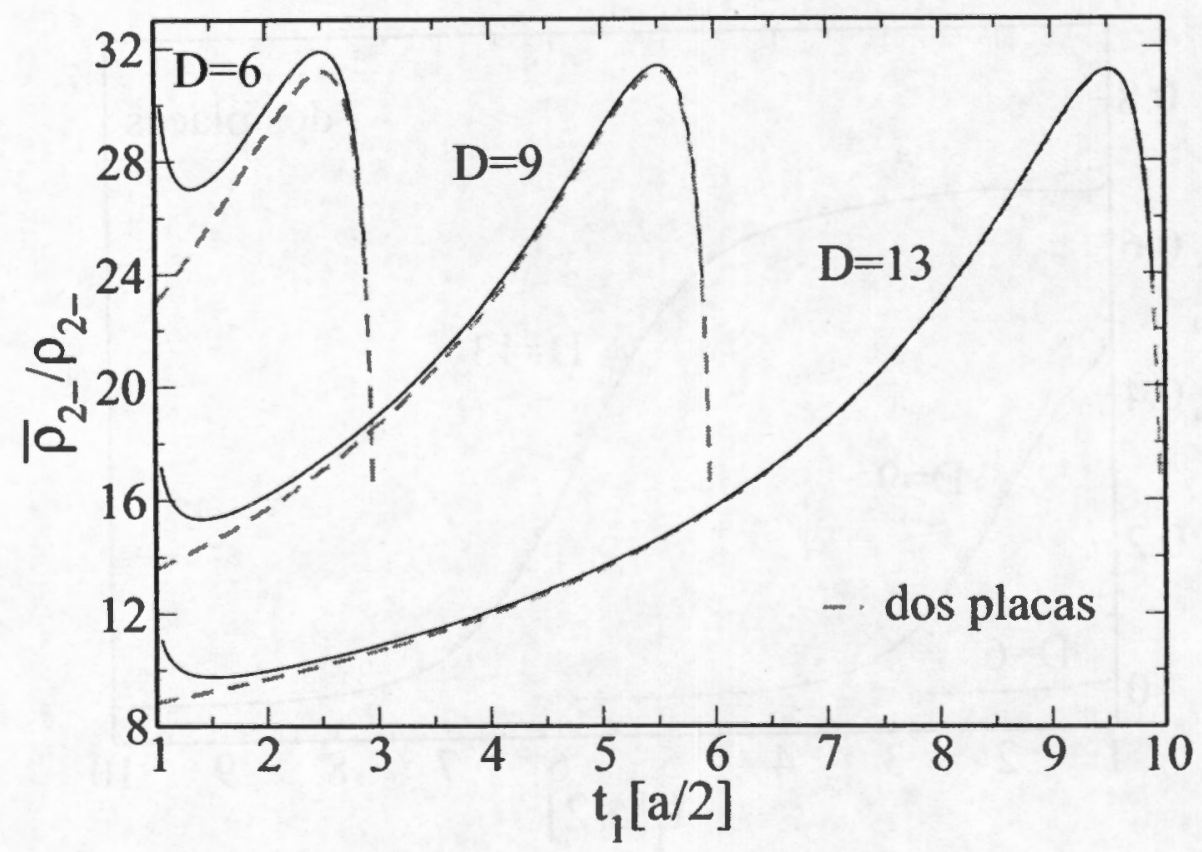

Figura G.15: Concentración promedio de contraiones dentro de la región II como una función de $t_{1},\left(\bar{\rho}_{2-} / \rho_{2-}\right)$ para las distancias fijas $D=t_{1}+d_{1}+t_{2}=6,9,13(a / 2)$. Para el caso monovalente como en la figura $\mathbf{3 . 1 3}$ 


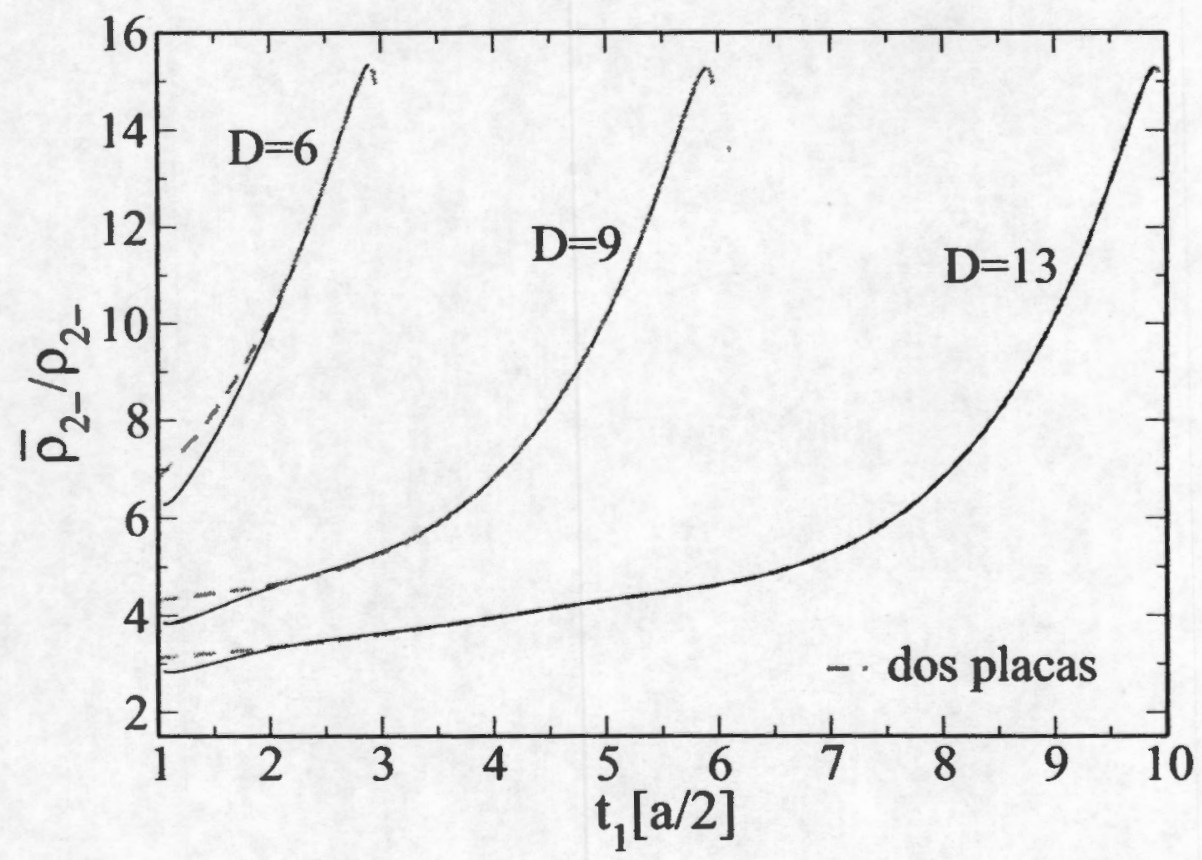

Figura G.16: Concentración promedio de contraiones dentro de la región II como una función de $t_{1},\left(\bar{\rho}_{2-} / \rho_{2-}\right)$ para las distancias fijas $D=t_{1}+d_{1}+t_{2}=6,9,13(a / 2)$. Para el caso divalente como en la figura 3.13 . 


\section{Bibliografía}

[1] P. C. Hiemenz and R. Rajagopalan, Principles of colloid and Surface Chemistry (Marcel Dekker, Inc, New York, 1997).

[2] R. J. Hunter, Introduction to Modern Colloid Science (Oxford University Press, Oxford, 1996).

[3] W. B. Russel, D. A. Saville, and W. R. Schowalter, Colloidal Dispersions (Cambridge University Press, Cambridge, 1989).

[4] K. S. Schmitz, Macroions in Solution and Colloidal Suspensions (VCH Publishers, New York, 1993).

[5] E. J. Verwey and J. T. G. Overbeek, Theory of stability of Lyophobic Colloids (Elsevier, Amsterdam, 1948).

|6| L. Guldbrand, B. Jönsson, and H. Wennerström, J. Colloid interface Sci. 89, 532 (1982).

[7] W. Hoppe, W. Lohmann, H. Markl, and H. Ziegler, Biophisics (Springer-Verlag, Berlin, 1983).

[8] J. H. Masliyah, Electrokinetic Transport Phenomena, 1st ed. ed. (AOSTRA, Alberta, Canada, 1994).

|9| W. Olivares, B. Sulbarán, and M. Lozada-Cassou, J. Phys. Chem. 97, 4780 (1993).

|10| E. González-Tovar, M. Lozada-Casscu, and W. Olivares, J. Chem. Phys. 94, 2219 (1991).

[11] E. González-Tovar and M. Lozada-Casscu, J. Physs. Chem. 93, 3761 (1989).

[12] M. Tasinkevych and A. Ciach, phys Rev. E 60, 7088 (1999).

[13] B. V. Derjaguin, N. V. Churajev, and V. M. Müller, Surface Forces (Consultants Bureau, New York, 1987), Vol. 7.

[14] J. N. Israelachvili and G. E. Adams, J. Chem. Soc.,Faraday Trans. 174, 975 (1979).

|15| R. G. Horn and J. N. Israelachvili, J. Chem. Phys. 75, 1400 (1981).

|16| P. Kékicheff and H. Christenson, Phys. Rer. Lett. 63, 2823 (1989). 
[17] P. Richetti, P. Kékicheff, J. L. Parker, and B. W. Ninham, Nature(london) 346, 252 (1990).

|18| D. A. Antelmi and P. Kékicheff, J. Pleys. Chem. B101, 8169 (1997).

[19] W. M.Gelbart, R. F.Bruinsma, P. A. Pincus, and V. A. Parsegian, Physics Today 53, 38 (2000).

[20] H. V. Olphen, An Introduction to Clay Colloid Chemistry (Wiley, N. Y., 1977).

[21] M. Lozada-Cassou, R. Saavedra-Barrera, and D. Henderson, J. Chem. Phys. 77, 5150 (1982).

[22] M. Lozada-Cassou, J. Chem. Phys. 80, 3344 (1984).

[23] M. Lozada-Cassou, Chem. Phys. 75, 1412 (1981).

[24] D. Henderson, M. Lozada, and L. Blim, J. Chem. Phys. 79, 3055 (1983).

[25] J. P. Valleau, L. K. Cohen, and D. N. Card, J. Phys. Chem. 72, 5942 (1980).

|26| M. Lozada-Cassou, J. Chem. Phys. 75, 1412 (1981).

[27] G. Müller and R. Brendel, Phys. Stat. Sol. A 182, 313 (2000).

[28] G. Schön and U. Simon, Coiloid Polvm. Sci. 273, 101,202 (1995).

|29| G. Keleman and G. Schön, J. of Materiai Science 27, 6036 (1992).

[30] A. Marziali and M. Akeson, Anni. Rav. Biomed. Eng. 3, 195 (2001).

[31] D.Routkevitch, A. Govyadinov, and P.Mardilovich, MEMS 2, 39 (2000).

[32] A. Eletskiř, Phisics-Uspekhi 45, 369 (2002).

[33| D. Henderson, L. Blum, and D. R. Smith, Chem. Phys. Lett. 63, 381 (1979).

[34] D. Henderson and L. Blum, J. Electroanal. Chem. 111, 217 (1980).

[35] M. Lozada-Cassou, R. Saavedra-Barrera, , and D., J. Chem. Phys. 77, 5150 (1982).

[36] R. D. Vold and M. J. Vold, Colloid end Interfece Chemistry (Addison-Wesley, Menlo Park Cal., 1983).

[37] D. A. McQuarrie. Statisticcl Mechurics (Harper-Collins publishers Inc., New York, 1976), review chapter.

[38] M. Lozada-Cassou and E. Díaz-Herrera, J. Chem. Phys. 92, 1194 (1990).

[39] M. Lozada-Cassou and E. Diaz-Herrera, J. Chem. Phys. 93, 1386 (1990). 
[40] M. Lozada-Cassou, in Fundamentals of Inhomogeneous Fluids, edited by D. Henderson (Marcel Dekker, New York, 1992), Chap. 8, fluids Between Walls and Pores.

[41] C. L. Rice and R. Whitehead, J. Phys. Chem 69, 4017 (1965).

[42] S. Levine, J. R. Marriott, G. Neale, and N. Epstein, J. Colloid Int. Sci. 52, 136 (1975).

[43] I. Watterson and L. White, J. Chem. Soc. Faraday Trans. II 77, 1115 (1981).

[44] W. B. Russel, J. Rheology 24, 287 (1980).

[45] H. L. Friedman, A Course in Statistical Mechanics (Prentice Hall and Englewood Cliffs, New Jersey, 1985).

[46] J. P. Hansen and I. R. McDonald, Theory of simple liquids, 2nd ed. (Academic Press, London, 1986).

[47] T. L. Hill, Statistical Mechanics (Dover Publications Inc., New York, 1987).

[48] L. S. Ornstein and F. Zernike, Proc. Akad. Sci. (Amsterdam) 17, 793 (1914).

[49] F. Zernike, Proc. Akad. Sci. (Amsterdam) 18, 1520 (1916).

|50| E. Waisman and J. L. Lebowitz, J. Chem. Phys. 52, 4307 (1970).

[51] D. Henderson, F. F. Abraham, and J. A. Barker, Molecular Physics 31, 1291 (1976).

152| D. Henderson, in Fundamentals of inhomogeneous fluids, edited by D. Henderson (Marcel Dekker, New York, 1993), Chap. 4.

[53| R. Kjellander and S. Marcelia, Mol. Phys. 83, 789 (1994).

[54] M. Lozada-Cassou, J. Chem. Phys. 75, 1412 (1981).

[55] M. Deserno, F. Jiménez-Ángeles, C. Holm, and M. Lozada-Cassou, J. Phỹs. Chem. B 105,10983 (2001).

[56] L. Degrève, M. Lozada-Cassou, E. Sánchez, and E. González-Tovar, J. Chem. Phys. 98, 8905 (1993).

[57] M. Lozada-Cassou, W. Olivares, and B. Sulbarán, Phys. Rev. E 53, 522 (1996).

[58] J. D. Jackson, Classical Electrodynamics (Wiley, New York, 1962).

|59| P. Debye and E. Hückel, Phisik. Z. 24, 185 (1923).

|60| G. Gouy, J. Phys. 9, 457 (1910).

[61] D. L. Chapman, Philos. Mag. 25, 475 (1913).

[62] J. G. Kirkwood, J. Chem. Phys. 2, 767 (1934). 
[63] W. Verbrugge and P. Pintauro, Traresport Models for Ion-Exchange Membranes, Modern Aspects of Electrochemistry (Plenum Press, New York, 1989), No. 19.

[64] M. Minor, A. J. van der Linde, and J. Liklema, J. Colloid Interface Sci. 203, 177 (1998).

[65] J. N. Israelachvili, Intermolecular and Surface Forces (Academic Press, New York, 1992).

[66] B. Smit, J. Phys. Chem. 99, 5597 (1995).

[67] G.Odriozola, F.Jimenez, and F. Guevara-Rodriguez, J. Phys. Chem. B 20, 2010 (2004).

[68] G. Hummer, J. C. Rasaiah, and J. P. Noworyta, Nature 414, 188 (2001).

[69] M. Sbai et al., J. Memb. Sci. 215, 1 (2003).

[70] S. S. Duhkin and B. V. Derjaguin, in Surface and Colloid Science, edited by E. Matijevic (Wiley-Interscience, New York, 1974), Vol. 7, review chapter.

171) L. Dresner and K. A. Kraus, J. Plyys. Chein 67, 990 (1963).

[72] L. Dresner and K. A. Kraus, J. Phys. Chem. 67, 990 (1963).

[73] D. Burgreen and F. R. Nakache, J. Phys. Chem. 68, 1084 (1964).

[74] D. Hildreth, J. Phys. Chem 74, 2006 (1976).

[75] S. Levine, J. R. Marriott, and K. Robinson, J. Chem. Soc. Faraday Trans. 2 71, 1 (1975).

[76] L. Dresner, J. Phys. Chem. 67, 1635 (1963).

[77] D. Coelho, M. Shapiro, J. F. Thover. and P. M. Adler, 3. Colloid Interface Sci. 181, 1699 (1996).

[78] A. Gupta, D. Coelho, and P. M. Alder, J. Colloid Interface Sci. 319, 549 (2008).

[79] M. Lozada-Cassou and E. Diaz-Herrera, J. Chem. Phys. 92, 1194 (1990).

|80| M. Lozada-Cassou and E. Diaz-Herreia, J. Chem. Phys. 93, 1386 (1990).

[81] L. Yeomans, S. E. Feller, E. Sánchez, and M.Lozada-Cassou, J. Chem. Phys. 98, 1436 (1993).

[82] G. E. Aguilar-Pineda, F. Jiménez-Angeles, J. Yu, and M. Lozada-Cassou, J. Phys. Chem. B 111, 2033 (2007).

[83] W. Olivares, B. Sulbarán, and M. Lczada-Cassou, J. Chen. Phys. 103, 8179 (1995).

[84] M. Deserno, F. Jiménez-Ángejes. C. Holm, and M. Lozada-Cassou, J. Phys. Chem. B 105, 10983 (2001). 
[85] W. Olivares, T. L. Croxton, and D. A. McQuarrie, J. Phys. Chem. 84, 867 (1980).

[86] W. Olivares and D. A. McQuarrie, J. Phys. Chem 89, 2966 (1985).

[87] M. Huerta and W. Olivares, J. Phys. Chem 91, 2975 (1987).

[88] V. L. Sigal and Y. Y. Ginsburg, J. Phys. Chem 85, 3735 (1981).

[89] V. Vlachy and D. A. McQuarrie, J. Phys. Chem. 90, 3248 (1986).

|90| V. Vlachy and A. D. J. Haymet, J. Am. Chem. Soc. 111, 477 (1989).

[91] M. Lozada-Cassou, J. Chem. Phys. 77, 5258 (1982).

|92] Z. Broz and N. Epstein, J. Colloid Int. Sci. 56, 605 (1976).

[93] E. Sánchez-Arellano, W. Olivares, M. Lozada-Cassou, and F. Jimenez-Ángeles, J. Coll. Int. Sci. 330, 474 (2009).

|94| J. Yu, L. Degrève, and M. Lozada-Cassou, Phys. Rev. Lett. 79, 3656 (1997).

[95] S. I. Stupp and P. V. Braun, Science 277, 1242 (1997).

[96] B. Jönsson et al., Langmuir 21, 9211 (2005).

[97] A. Nonat, Cem. Concr. Res. 34, 1521 (2004).

[98| A. C. D. Newman, Chemistry of Clays and Clay Minerals (Mineralogical Society, London, 1987).

[99] F. C. Chang, N. T. Skipper, and G. Sposito, Langmuir 11, 2734 (1995).

[100] F. C. Chang, N. T. Skipper, and G. Sposito, Langmuir 14, 1201 (1998).

[101] M. Chavez-Paez, L. de Pablo, and J. de Pablo, J. Chem. Phys. 24, 10948 (2001).

|102| E. Park and G. Sposito, J. Phys. Chem.B 104, 4648 (2000).

[103| E. S. Boek and M. Sprik, J. Phys. Chem. B 107, 3251 (2003).

|104| E. S. Boek, P. V. Coveney, and N. T. Skipper, J. Amm. Chem. Soc. 117, 12608 (1995).

|105] F. C. Chang, N. T. Skipper, and G. Sposito, Langmuir 13, 2074 (1997).

[106] R. Sutton and G. Sposito, J. Colloid Interface Sci. 237, 174 (2001).

[107] D. A. Young and D. E. J. Smith, J. Phys. Chem. B 104, 9163 (2000).

|108| V. Marry and P. Turq, J. Phys. Chem. B 107, 1832 (2002).

|109| N. T. Skipper, G. D. Williams, A. V. C. D. Siqueira, and C. Lobban, Clay Miner. 35, $283(2000)$. 
|110| K. Norrish, Discuss. Faraday Soc. 18, 120 (1954).

[111] C. E. Marshall, The Colloid Chemistry of the Silicate Minerals (Academic, N Y, 1949).

[112] H. V. Olphen, in in Chemistry of Clays and Clay Minerals, No. 6 in Mineralogical Society of London, edited by A. C. D. Newman (Wiley, New York, 1987), .

[113] H. H. et al., Chem. mater 19, 59 (2007).

[114] K. M. Chv́ez-Páez, L. d. P. Van Workum, and J. J. de Pablo, J. Chem. Phys. 114, 1405 (2001).

[115] E. J. M. Hensen and B. Smit, J. Phys. Chem. B 106, 664 (2002).

|116| G. Odriozola and F. de J. Guevara-Rodríguez, Langmuir 20, 2010 (2004).

[117] D. E. Smith, Y. Wang, and H. D. Whitley, Fluid Phase Equilib. (PUBLISHER, ADDRESS, 2004), pp. 222--223, 189-194.

[118] H. D. Whitley and D. E. J. Smith, J. Chem. Phys. 120, 5387 (2004).

[119] F. Chang et al., in ACS Symposium, 715, edited by L. Schultz, H. van Olphen, and F. Mumpton (American Chemical Society, Washington, DC, 1999).

[120| E. J. M. Hensen, T. J. Tambach, A. Bliek, and B. Smit, J. Chem. Phys. 115, 3322 (2001).

[121] T. J. Tambach, E. J. M. Hensen, T. J. Tambach, and B. Smit, J. Phys. Chem.B 108, 7586 (2004).

[122] G. Sposito et al., Greathouse, J. Proc. Natl. Acad. Sci. 96, 3358 (1999).

|123| G. Odriozola, J. F. Aguilar, and J. Lopez-Lemus. J. Chem. Phys. 121, 4266 (2004).

[124| C. E. Weaver and L. D. Pollard, The Chemistry of Clay Minerals (Elsevier, Amsterdam, 1973).

|125| J. M. Saunders, J. W. Goodwin, R. M. Richardson, and B. Vincent, J. Phys. Chem.B 103, 9211 (1999).

[126] J. C. P. Gabriel, C. Sanchez, and P. Davidion, J. Phys. Chem. 100, 139 (1996).

[127] A. Mourchid et al., Langmuir 14, 4718 (1998).

|128| T. Nicolai and S. Cocard, Langmuir 16, 8189 (2000).

|129| M. Kroon, G. H. Wegdam, and R. Sprik, Europhys. Lett. 35,-621 (1996).

|130| S. Kutter, J. P. Hansen, M. Sprik, and E. Boek, J. Chem. Phys. 112, 311 (2000).

[131] M. Dijkstra, J. P. Hansen, and P. A. Madden, Phys. Rev. Lett. 75, 2236 (1995). 
[132] R. Kjellander, S. Marcelja, R. M. Pashley. and J. P. Quirk, J. Chem.Phys. 92, 4399 (1990).

[133| R. Pellenq, J. Caillot, and A. J. Delville, J. Phys. Chem.B 101, 8584 (1997).

[134] J. M. Pellenq, A. Delville, and H. van Damme, in In Characterisation of Porous Solids, edited by B. M. et al. (PUBLISHER, Cambridge U. K., 1997), Chap. IV.

[135] H. V. Damme, in In Encyclopedia of Surface and Colloid Science, edited by B. M. et al. (Marcel Dekker, New York, 2002).

[136] A. Delville, R. Pellenq, and J. Caillot, J. Chem. Phys. 106, 7275 (1997).

[137] A. V. Richard, R. K. Teukolsky, and E. P. Giannelis, Chem. Mater 6, 1017 (1994).

[138] S. Geoffrey and L. Barden, Can Geotech J. 8, 391 (1971).

[139] J. Israelachvili and H. Wennerström, Nature 379, 219 (1996).

[140| B. S. Krishna, N. Mahadevaiah, D. S. R. Murty, and B. S. J. Prakash, J. Colloid Interf. Sci. 271, 270 (2003).

[141] J. N. Israelachvili and P. M. McGuiggan, Science 241, 795 (1988).

[142] P. T. Tanev and T. J. Pinnavai, Science 271, 1267 (1993).

[143| C. R.Safinya et al., Science 261, 588 (1993).

[144] J. O. Rädler, I. Koltover, T. Salditt, and C. R. Safinya, Science 275, 810 (1997).

[145] F. Namouni and C. Porco, Nature 417, 45 (2002).

[146] H. Rauch, H. Lemmel, M. Baron, and R. Loidl, Nature 417, 630 (2002).

[147] N.E.Levinger, Science 298, 1722 (2002).

[148] J. N. Israelachvili and D. Guordon, Science 292, 867 (2001).

[149] F. Jiménez-Ångeles, G. Odriozola, and M. Lozada-Cassou, J. Phys.: Condens. Matter 18, 2335 (2006).

[150] S. Zhu and et al, Nature (London) 400, 49 (1999).

[151] F. Jiménez-Ángeles, G. Odriozola, and M. Lozada-Cassou, J. Phys.: Condens. Matter 21, 424107 (2009).

[152] G. Agular-Pineda, F. Jiménez-Ángeles, J. Yu, and M. Lozada-Cassou, J. Phys. Chem. B 111, 2033 (2007).

[153] G. Odriozola, F. Jiménez-Ángeles, and M. Lozada-Cassou, PRL 97, 18102 (2006).

[154] A. Tulpar, P.R.Tassel, and J.Waltz, Langmuir 22, 2876 (2006). 
|155| J.C.Crocker and D.Grier, Phys. Rev. Lett. 77, 1897 (1996).

[156] G.M.Kepler and S.Fraden, Phys. Rev. Lett. 73, 356 (1994).

[157] Y. Han and D. Grier, Phys. Rev. Lett. 91, 038302 (2003).

[158] K. W. et al., Langmuir 16, 303 (2000).

[159] C. M. W. et al., Macromolecules 37, 4974 (2004).

[160| C. R. Safinya, curr. Opin. Struct. Biol. 11, 440 (2001).

[161] H. Liang, T. E. Angelini, P. V. Braun, and G. C. Wong, J. AM. Chem. Soc. 126, 157 (2004).

|162| B. D. y L. Landau, Acta Physicochim. URSS 14, 633 (1942).

[163] R. Kjellander, J. Chem. Phys. 88, 7129 (1988).

[164] E. C. Nelson and P. V. Braun, Science 318, 924 (2007).

|165| S. L. Carnie, D. Y. C. Chan, D. J. Mitchel, and B. W. Ninham, J. Chem. Phys. 74, 1472 (1981).

|166| S. L. Carnie and D. Y. C. Chan, Chem. Phys. Lett. 77, 437 (1981).

[167] M. Lozada-Cassou, R. Saavedra-Barrera, and D. Henderson, J. Chem. Phys 77, 5150 (1982).

[168] M. Lozada-Cassou and D. Henderson, J. Phys. Chem. 87, 2821 (1983).

|169| S. L. Carnie and G. M. Torrie, in In Advances in Chemical Physics, edited by I. Preigogine and S. A. Rice (Wiley, New York, 1984).

|170| L. Blum, Molec. Phys. 30, 1529 (1975).

|171| E. Waisman and J. L. Lebowitz, J. Chem. Phys. 56, 3086 (1972).

|172] T. Croxton and D. McQuarrie, J. Phys. Chem. 83, 1840 (1979).

[173| T. Croxton, , and D. McQuarrie, Mol. Phys 42, 141 (1981).

[174] K. Hiroike, J.Phys. Soc. Jpn 27, 1415 (1969).

[175] K. Hiroike, Mol. Phys 33, 1195 (1977).

|176| C. W. Outhwaite and L. B. Bhuivan, J. Chem. Soc. Faraday Trans. 76, 1388 (1981).

[177] C. W. Outhwaite and L. B. Bhuiyan. J. Chem. Soc. Faraday Trans. 79, 707 (1983).

[178] J. A. Barker and D. Henderson, Review fo Modern Phys. 48, 998 (1976).

[179] M. Lozada-Cassou, J. Chem. Phys. 87, 3279 (1983). 
[180] M. Lozada-Cassou and J. Yu, Phys. Rev. Lett. 77, 4019 (1996).

[181] M. Lozada-Cassou and J. Yu, Phys. Rev. Lett. 56, 2958 (1997).

[182] M. Lozada-Cassou, W. Olivares, B. Sulbarán, and Y. Jiang. Physica A 231, 197 (1996).

[183] F. Jiménez-Ángeles, Ph.D. thesis, Liniversidad Autónoma Metropolitana-Iztapalapa, Mexico, 1996.

[184] G. Strang and G. Fix, An Analysis of the Finite Element Method (Prentice Hall and Englewood Cliffs, New Jersey, 1973).

[185] G. Pinder and W. Gray, Finite Element Simulation in Surface and subsurface Hidrology (Academic Press Inc., London, 1977).

|186| E. Becker, G. Carey, and J. Oden, Finite Element:An Introduction (Prentice Hall, Englewood Cliff, N. J., 1973), Vol. 1. 
